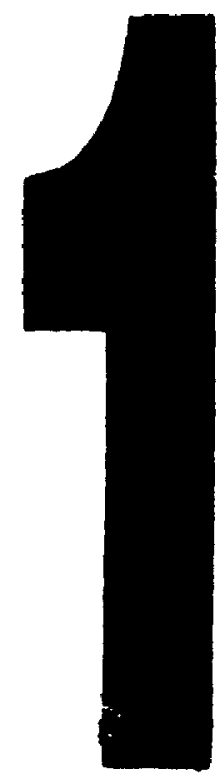

PM-1 31\%" $\times 4$ " PHOTOGRAPHIC MICROCOPY TARGET NES 1010a ANSIIISO *2 EOUIVALENT

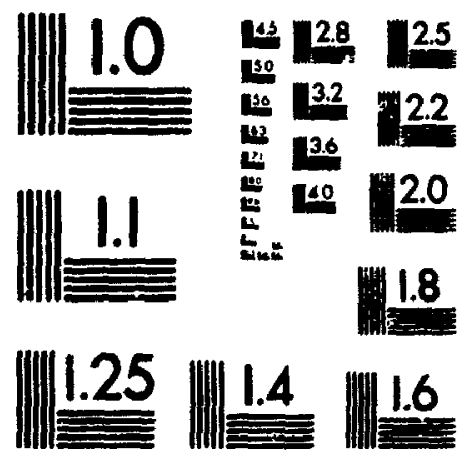

PRECISION RESOLUTION TARGETS 
National Library

of Canada

Acquisitions and

Bibliographic Services Branch

395 Wellington Street

Ottawa. Ontario

K1A ON4
Bibliotheque nF'ionale

du Canada

Direction des acquisitions et

des services bibliographiques

395, rue Wcllington

Otlawa (Ontario)
The quality of this microform is heavily dependent upon the submitted for microfilming. Every effort has been made to ensure the highest quality of reproduction possible.

If pages are missing, contact the university which granted the degree.

Some pages may have indistinct print especially if the original pages were typed with a poor typewriter ribbon or if the university sent us an inferior photocopy. quality of the original thesis

La qualité de cette microforme dépend grandement de la qualité de la thèse soumise au microfilmage. Nous avons tout fait pour assurer : ve qualité supérieure de reproduction.

S'il manque des pages, veuillez communiquer avec l'université qui a conféré le grade.

La qualité d'impression de certaines pages peut laisser à désirer, surtout si les pages originales ont été dactylographiées à l'aide d'un ruban usé ou si l'université nous a fait parvenir une photocopie de qualité inférieure.

Reproduction in full or in part of this microform is governed by the Canadian Copyright Act, R.S.C. 1970, C. C-30, and subsequent amendments.
La reproduction, même partielle, de cette microforme est soumise à la Loi canadienne sur le droit d'autour, SRC 1970, c. C-30, et ses amendements subséquents. 


\title{
Probing Anomalous Contributions to Triple Boson Couplings
}

by

Paul E. Madsen, B.Sc., M.Sc.

\author{
A thesis submitted to \\ the Faculty of Graduate Studies and Research \\ in partial fulfilment of \\ the requirements for the degree of \\ Doctor of Philcsophy
}

Department of Physics

Ottawa-Carleton Institute for Physics

Ottawa, Ontario

November, 1994

(C) copyright 1994, Paul E. Madsen 
National Library

of Canada

Acquisitions and Bibliographic Services Branch

395 Welington Streel Ottewa, Oritario

K1AON
Bibliotheque nationale

du Canada

Direction das acquisitions ef

des senvices bibliographiques

395. no Willinotion

Ortama (Cnesio)

KIA ON
Your the Volmo nuturnes

Our the Nowenturence
THE AUTHOR HAS GRANTED AN IRREVOCABLE NON-EXCLUSIVE LICENCE ALLOWING THE NATIONAL LBRARY OF CANADA TO REPRODUCE, LOAN, DISTRIBUTE OR SELL COPIES OF HIS/HER THESIS BY ANY MEANS AND IN ANY FORM OR FORMAT, MAKING THIS THESIS AVAILABLE TO INTERESTED PERSONS.
L'AUTEUR A ACCORDE UNE LICENCE IRREVOCABLE ET NON EXCLUSIVE PERMETTANT A LA BIBLIOTHEQUE NATIONALE DU CANADA DE REPRODUIRE, PRETER, DISTRIBUER OU VENDRE DES COPIES DE SA THESE DE QUELQUE MANIERE ET SOUS QUELQUE FORME QUE CE SOIT POUR METTRE DES EXEMPLAIRES DE CETTE THESE A LA DISPOSITION DES PERSONNE INTERESSEES.
THE AUTHOR RETANNS OWNERSHIP

OF THE COPYRIGHT IN HIS/HER THESIS. NEITHER THE THESIS NOR SUBSTANTIAL EXTRACTS FROM IT MAY BE PRINTED OR OTHERWISE REPRODUCED WITHOUT HIS/HER PERMISSION.
L'AUTEUR CONSERVE LA PROPRIETE DU DROIT D'AUTEUR QUI PROTEGE SA THESE. NI LA THESE NI DES EXTRAITS SUBSTANTIELS DE CELLECI NE DOIVENT ETRE IMPRIMES OU AUTREMENT REPRODUITS SANS SON AUTORISATION.

ISBN $\quad 0-315-98510-0$ 
Name

MADSEN

Dissernation Abstracts Internotional is arranged by broad, general subject categories. Please select the one subject which most nearly describes the content of your dissertation. Enter the corresponding four-digit code in the spaces provided.

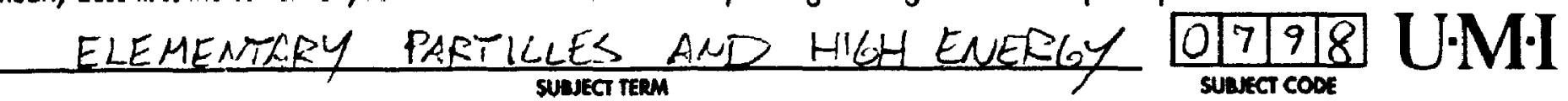

\section{Subject Colegories}

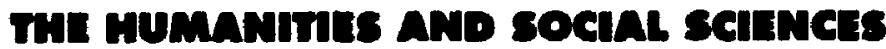

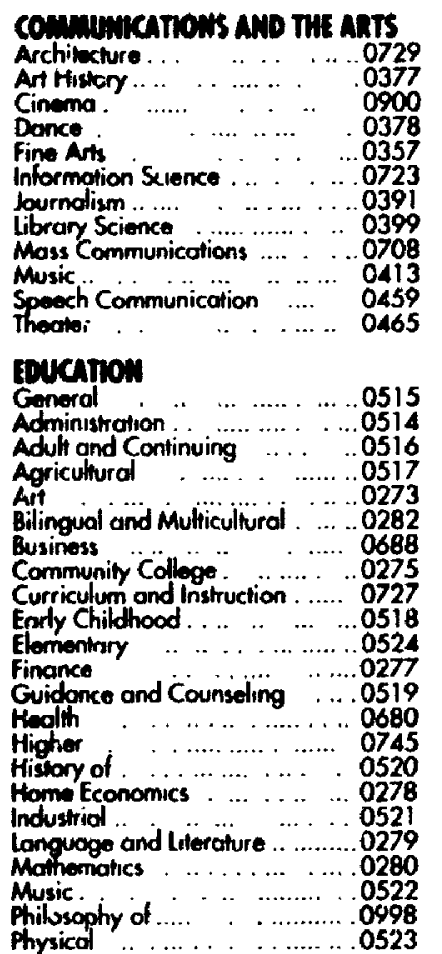

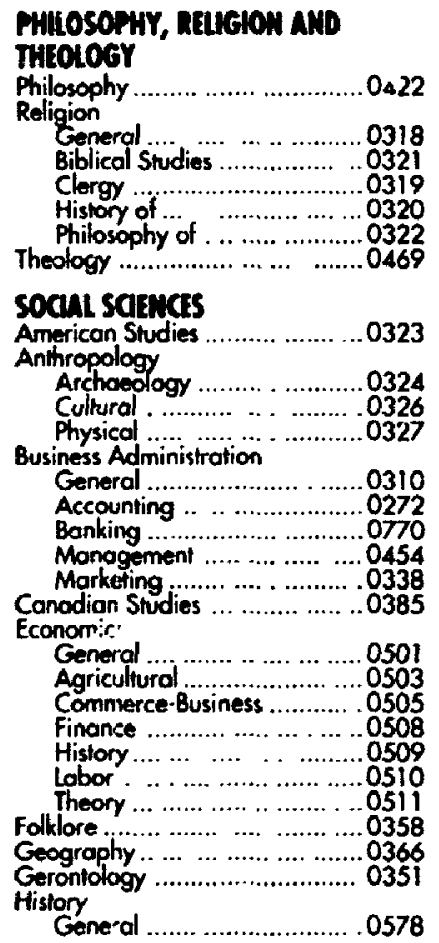

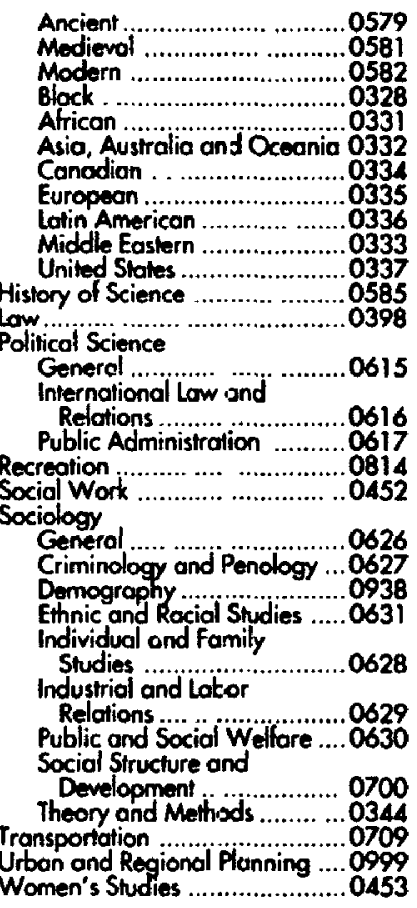

Engineering

Generd ........................0537 Aerospace .......................... 0538 Agricultural .......................... 0539 Automotive ........................ 054 Chemical ........................0542 Civil ... .......................... 0543 Electronics and Electrical ...... 0544 Heat and Thermodynamics... 0348 Hydraulic ........................ 0545 Industrinl ....................... O546

Marine ........................... 0794

Mechanical .......................... O548

Mechanical ..................... 0548

Mining ............................0551

Nucleor ............................. 0552

Pockgging .....................0549

Petroleum ....................0765

Sanitary and Municipal ......0554

System Science ... ... .............0790

Geotechnology .........................0428

Operations Research ...................0796

Plastics Jechnology .................... 0795

Textile Technology

0994

\section{PSYchotegY}

Generd ..........................0621

Behovioral ............................. 038

Clinical ...............................0622

Developmental .....................0620

Experimental .. ......................0623

Personality..............................0625

Personality ............................... 0989

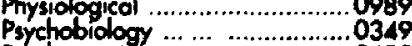

Psychometrics ............................. 0632

Socid ..................................0451
Ginith satinas

Gochemestry
Applied Sciences

Applied Mochanics ........... .....0346

Computer Science ....................0984 
The undersigned hereby recommend to the Faculty of Graduate Studies and Research acceptance of the thesis

\section{"PROBING ANOMALOUS COUPLINGS TO TRIPLE BOSON COUPLINGS" submitted by \\ PAUL EDWARD MADSEN, B.Sc., M.Sc.}

in partial fulfilment of the requirements

for the degree of Doctor of Philosophy
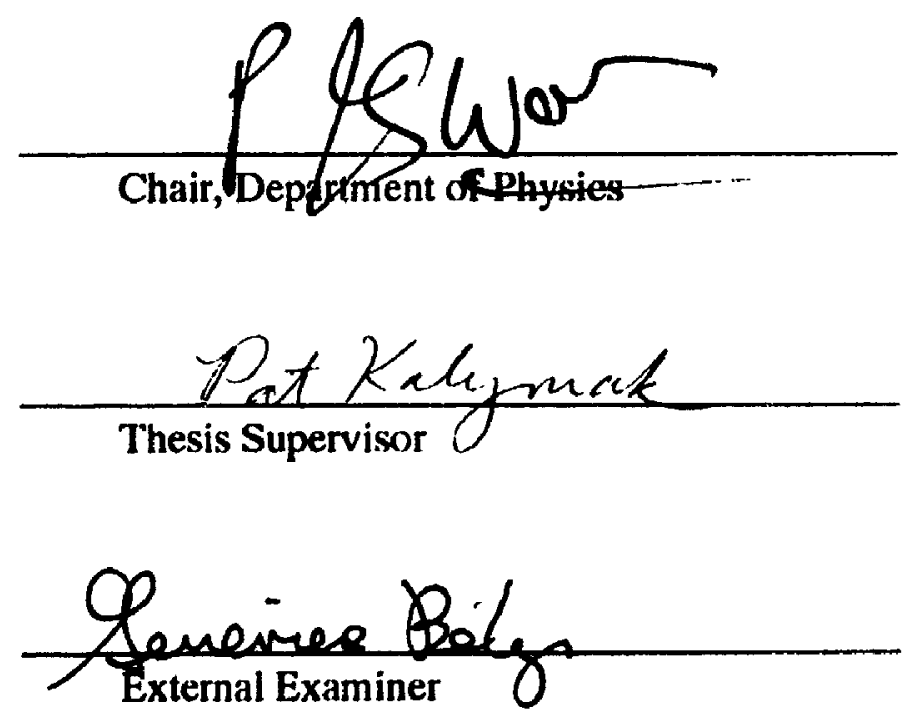

\section{Carleton University}

November 2, 1994 


\section{Abstract}

The Standard Model of the unified electroweak interactions (SM) has been confirmed by all experiments to date. This empirical evidence for the validity of the theory has been almost entirely restricted to the interactions between the rnatter particles, the fermions, and the forcemediating gauge particles, the bosons. However, a fundamental prediction of the SM is that the gauge bosons should also interact amongst themselves; both trilinear and quartic couplings are predicted. These boson self-interactions are a consequence of the non-abelian nature of the electroweak gauge group and are essential for both the unitarity and renormalizability of the theory. Future colliders like LEP2, $e^{+} e^{-}$at $\sqrt{s}=190 \mathrm{GeV}$ and the $\mathrm{NLC}, e^{+} e^{-}$at $\sqrt{s}=500$ $\mathrm{GeV}$, will make possible direct tests of the gauge self-couplings by the measurement of processes which get tree level contributions from these vertices.

This thesis describes the investigation of the triple gauge boson couplings $W W \gamma$ and $W W Z$ through the four lepton production processes

$$
e^{+} e^{-} \rightarrow l^{+} \nu l^{-} \bar{\nu}
$$

where we consider all possible final state lepton configurations. The analysis is performed at the energies of $\sqrt{s}=500 \mathrm{GeV}$ and $\sqrt{s}=1 \mathrm{TeV}$. By examining the sensitivity of the total crosssections and experimental distributions of the above processes to variations in the triple boson vertex parameters, in particular the anomalous magnetic moment parameter $\kappa_{V}$ and the $C P$ violating $\bar{\kappa}_{v}$ and $\bar{\lambda}_{v}$, we determine the detection potential of these processes for such deviations.

Assuming an integrated luminosity of $50 \mathrm{fb}^{-1}$ for these future colliders, we find that the annmalous magnetic moment parameter $\kappa_{V}$ could be measured to within the order of a few percent, $\pm(2-3) \%$ of its Standard Model value; the higher energy of $1 \mathrm{TeV}$ can improve the constraints by a factor of 2 for certain of the processes. Limits of this level are a significant imprcivement on the bounds of $\sim \pm 10 \%$ predicted for LEP2 and probe both the loop corrections to the vertices as well as the corrections predicted by certain extensions to the Standard Model. Independent constraints on $\kappa_{\gamma}$ and $\kappa_{z}$ might be determined by the implementation of suitable phase space cuts; these independent limits are of the order of $\pm 20 \%$ for $\kappa_{Z}$ and $\pm 10 \%$ for $\kappa_{\gamma}$. A polarized beam facility would access the rich helicity structure of the processes; with such 
information we could improve both the limits on $\mathrm{Ar}$ as well as the ability to disicriminate herwen $\kappa_{\gamma}$ and $\kappa_{z}$.

The CP violating couplings $\dot{\kappa}_{y}$ and $\dot{\lambda}_{y}$. could be measured to within limits of the ordier of \pm() . 15 and \pm 0.005 respectively. The "purest" signature of such $(P$ violation would be asymmetriess in event rates between CP conjugate states; we find that, for $\dot{n}_{1} \cdot \dot{\lambda}_{1}=1$, the oxpected magnitule for such asymmetries in the total cross section is below the statistiral significance lew of $1-2 \%$. The asymmetries in the individual helicity amplitude contributions to the total cross section call however exceed this significance level; polarized $r^{+} e^{-}$beams would be necessary to explore this effect. 


\section{Acknowledgements}

Sincere thanks go to my supervisor Pat Kalyniak for her guidance and friendship throughout my years at Carleton. For her patience, both when I missed NSERC deadlines and in thoroughily editing this manuscript, and for teaching me, amongst other things, that $\Lambda$ and $\Gamma$ are different, I am grateful.

I would have been lost along the way were it not for the Physics Department secretaries to guide me. They led me through the maze of paperwork, copiers, and fax machines that "higher education" seems to consist of. Thanks to Teresa Buckley, Eva Lacelle, and especially the late Roselyn Tighe; every grad student should have someone like Roz looking out for them.

To my parents, for their encouragement, both in word and deed, I will be forever in debt (emotionally if not financially).

To my wife Julie, for her curiosity, and for learning to hide her smile when I said that I would be finished in a few months, my love and gratitude. 


\section{Contents}

List of Tables $\quad$ vi

List of Figures $\quad$ vii

1 Introduction to Electroweak Interactions 2

1.1 Historical Development of Electroweak Theory . . . . . . . . . . . . . 2

1.2 Symmetry and Gauge Theories . . . . . . . . . . . . . . . 4

$1.3 S U(2)_{L} \times U(1)_{Y}$ as a local symmetry $\ldots \ldots \ldots \ldots \ldots$ is

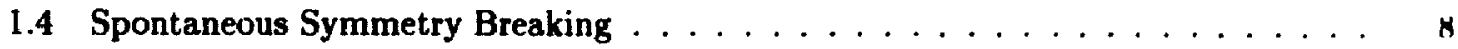

1.5 Spontaneous Breaking of $S U(2)_{L} \times U(1)_{\gamma} \ldots \ldots \ldots \ldots \ldots$

1.6 Problems with the Standard Model . . . . . . . . . . . . . . I4

2 Theoretical Background 17

2.1 Triple Boson Vertex $\ldots \ldots \ldots \ldots \ldots \ldots \ldots \ldots$

2.2 Previous Research $\ldots \ldots \ldots \ldots \ldots \ldots \ldots$

2.3 Motivation for Studying $e^{+} e^{-} \rightarrow t^{+} \nu l^{-}-\bar{\nu} \ldots \ldots \ldots \ldots \ldots$

3 Four Lepton Production Processes 26

3.1 Feynman Diagrams . . . . . . . . . . . . . . . . . . 26

3.2 Helicity Amplitudes $\ldots \ldots \ldots \ldots \ldots \ldots \ldots$

3.3 The Calculation $\ldots \ldots \ldots \ldots \ldots \ldots \ldots \ldots \ldots \ldots \ldots \ldots$

4 Deriving Limits for $\kappa_{V}$

4.1 Limits from total cross-section measurements $\ldots \ldots \ldots \ldots \ldots$

4.1.1 Effect of Including Systematic Uncertainties . . . . . . . . . . . . . . 49 
4.1 .2 Scaling Behaviour of Sensitivities . . . . . . . . . . . 50

4.2 Distributions as Indicators of Non-Standard Vertex Couplings . . . . . . . . . 52

4.3 Improving Sensitivity Through Cuts . . . . . . . . . . . . 58

4.4 Discriminating Between $\gamma \mathrm{WW}$ and $\mathrm{zWW} \ldots \ldots \ldots \ldots \ldots$

4.4 .1 Independent $\kappa_{z}$ Limits. . . . . . . . . . . . . . 66

4.4 .2 Independent $\kappa_{\gamma}$ Limits . . . . . . . . . . . . . 69

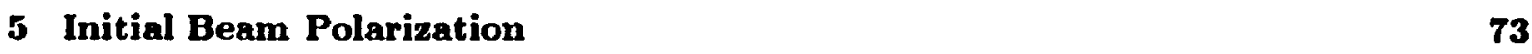

5.1 Improving $\kappa_{v}$ bounds Through Polarization Studies . . . . . . . . . 74

5.2 Polarization as means of Differentiating between $\kappa_{y}$ and $\kappa_{Z} \ldots \ldots \ldots \ldots$

6 Form Factor Modifiration of Anomalous Couplings $\quad 87$

7 CP Violating Triple Vertex Contribution $\quad 94$

7.1 CP violation in Triple Gauge Boson Vertices . . . . . . . . . . . . . . . 96

7.2 Sensitivity Limits on $\tilde{\kappa}_{V}$ and $\tilde{\lambda}_{V} \ldots \ldots \ldots \ldots$

7.2.1 Total Cross-Section Limits $\ldots \ldots \ldots \ldots \ldots . \ldots . \ldots . \ldots$

$7.2 .2 x^{2}$ Analysis . . . . . . . . . . . . . . . 100

7.3 Distributions as Indicators of CP violation . . . . . . . . . . . 103

7.4 Asymmetries as Indicators of CP Violation . . . . . . . . . . . . . 105

7.4 .1 CP-odd variables . . . . . . . . . . . . . . . . 107

7.4.2 Asymmetries in $S$ and $Q \ldots \ldots \ldots \ldots \ldots$

$\begin{array}{lr}\text { A Feynman Rules } & 115\end{array}$

B Full Set of Contributing Diagrams $\quad 117$

$\begin{array}{ll}\text { C CALKUL Formalism } & 122\end{array}$

$\begin{array}{ll}\text { D Amplitude Expressions } & 126\end{array}$

E Monte Carlo Algorithm 


\section{List of Tables}

4.1 $95 \%$ C.L. bounds on non-standard couplings $\kappa_{v}$ about Standard Model $\kappa_{v}=1.0$

4.2 Cross-sections and ratios relative to the $\mathrm{SM}$ for different angular cuts for $\mu \tau$ proress at $500 \mathrm{Gev} \ldots \ldots \ldots \ldots \ldots \ldots \ldots$. . . . . . . . . . . . . .

$4.32 \sigma$ limits on $\Delta \kappa_{V}$ for various angular cuts for $\mu \tau$ process at 500 ( iaV. . . . . . (i)

$4.42 \sigma$ limits for various cut combinations for $\mu^{+} \tau^{-}$process at $500 \mathrm{GeV} \ldots \ldots \ldots$

$7.195 \%$ C.L. bounds on non-standard couplings $\tilde{n} v \ldots \ldots \ldots$

$7.295 \%$ C.L. bounds on non-standard couplings $\bar{\lambda}_{V} \ldots \ldots \ldots$

$7.3 x^{2} 95 \%$ C.L. bounds on non-standard couplings $\tilde{k}_{V}$. Limits are taken from $l^{2}$ as

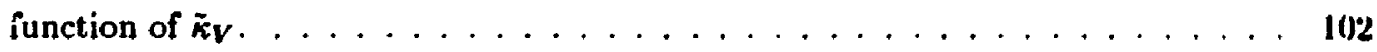

$7.4 \chi^{2} 95 \%$ C.L. bounds on non-standard couplings $\bar{\lambda}_{v}$. Limits are taken from $\gamma^{2}$ as function of $\tilde{\lambda}_{v} \ldots \ldots \ldots \ldots \ldots \ldots$ 10s

7.5 Asymmetries $A_{C P}^{S}\left(\times 10^{3}\right)$ in Tctal Cross-Section $\ldots \ldots \ldots \ldots$ 


\section{List of Figures}

1.1 Potential as function of $\phi$ for $\boldsymbol{\mu}^{2}>0 \ldots \ldots \ldots$

1.2 Potential as function of $\phi$ for $\mu^{2}<0 \ldots \ldots \ldots \ldots$

1.3 Triple Gauge Boson Vertex $\ldots \ldots \ldots \ldots \ldots$

2.1 The tree level Feynman diagram for $Z$ boson coupling to fermions (a), and the loop diagram involving the $\mathrm{ZWW}$ vertex (b) . . . . . . . . . . . . . 20

2.2 Feyman diagrams that contribute to $W$-pair production. The triple boson vertex is indicated by the shaded circle. . . . . . . . . . . . . . . . . .

3.I Triple boson vertex dependent diagrams that contribute to all pcssible four lepton final state configurations. . . . . . . . . . . . . . . . 27

3.2 Vertex independent diagrams that contribute to all four possib! four lepton final state configurations . . . . . . . . . . . . . . . . 27

3.3 Vertex dependent diagrams that contribute to (a) $\mu^{+} e^{-}$and $e^{+} e^{-}$configurations and (b) $e^{t} e^{-}$configuration. . . . . . . . . . . . . . . .

3.4 Vertex independent diagrams that contribute to the $\mu^{+} e^{-}$and $e^{+} e^{-}$final state configurations . . . . . . . . . . . . . . . . . 28

3.5 Vertex dependent diagrams that contribute to the $\mu^{+} \mu^{-}$and $e^{+} e^{-}$states. . . . 29

3.6 Vertex independent diagrams that contribute to $\mu^{+} \mu^{-}$and $e^{+} e^{-}$states. . . . . 29

3.7 Vertex independent diagrams that contribute only to the $e^{+} e^{-}$final state configuration. . . . . . . . . . . . . . . . . . . . 30

4.1 Cross-section as function of center-of-mass energy $\ldots \ldots \ldots \ldots \ldots$

4.2 Ratio of non-standard to standard cross-section as function of $\kappa_{\gamma}$ and $\kappa_{Z}$ at 500 GeV. . . . . . . . . . . . . . . . . . . . 
4.3 Ratio of non-standard to standard cross-section as function of $x$, and $n$ \% at llle(l)

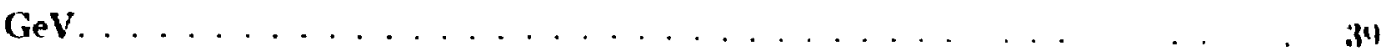

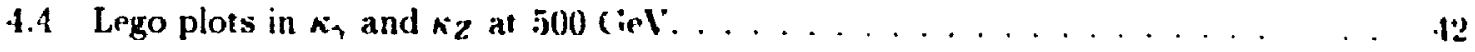

4.5 Lego plots in $\kappa_{\gamma}$ and $\kappa_{z}$ at 1 TeV . . . . . . . . . . . . . .

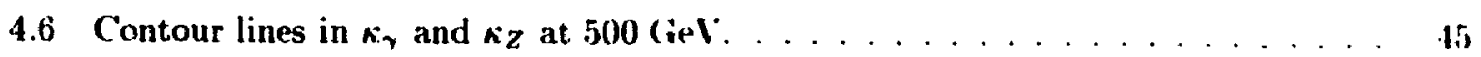

4.7 Contour lines in $\kappa_{\gamma}$ and $\kappa_{z}$ at $1 \mathrm{TeV} . \ldots \ldots \ldots \ldots$

4.8 Differential cross-sections with respert to (a) $\cos \theta_{r-},(b) \cos \theta_{\mu+}$, and (c) coss $\theta_{\mu r}$. 5.1

4.9 Differential cross-section with respect to $\cos \theta_{r-}$ for $\mu^{+} r^{-}$procens at. 500 (ieV. . 5.4

4.10 Differestial cross-section with respect to $x_{-}$for the $\mu^{+} \tau^{-}$process at 5010 (ieV. . 55)

4.11 Differential cross-section with respect to $M_{\mu}$ for the $\mu^{+} \tau^{-}$prowess att 5010 (ieV . 51i

1.12 Differential cross-section with respect to $E_{\text {vis }}$ for the $\mu^{+} r^{-}$proress at 5010 (ieV . 5,i

413 Differential cross-section with respect to $M_{\mu \mu}$ for the $\mu^{+} \mu^{-}$process at. 500 ( $\mathrm{irV}$. 57

4.14 Differential cross-section with respect to $P_{T}$ for the $\mu^{+} \mu^{-}$channels al. 500 (irV . 5,

4.15 Distributions in invariant mass $M_{\mu \tau}$ for $\mu^{+} \tau^{-}$channel at 500 (ieV for dilferent. angular cuts. ......................

4.16 Sensitivity Ratios for $\mu^{+} \mu^{-}$process at $500 \mathrm{GeV}$. when $M_{\mu \mu}$ restricted $\alpha M_{z} \pm 5$

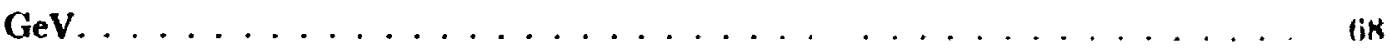

$4.172 \sigma$ contour for $\mu^{+} \mu^{+}$process at $500 \mathrm{GeV}$ when $M_{\mu \mu}$ restricted to $M_{\%} \pm 5$ (ir.V. . (ij)

4.18 Cross-section as function of $\kappa_{V}$ for $\mu^{+} e^{-}$channel at 500 ( ieV with $-0.9<\cos 00_{c-}<$

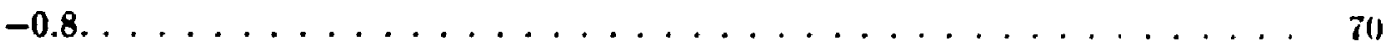

$4.192 \sigma$ contours in $\kappa V$ for the $\mu^{+} e^{-}$channel at 500 ( GeV with $-0.9<\cos \theta_{e-}<-0.8 . \quad 71$

5.1 Cross-section as function of $\kappa_{\gamma}$ and $\kappa_{z}$ for $(a)(+-+-)$ and (b)(-++-) hrelirity an1-

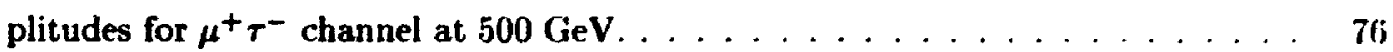

5.2 Helicity amplitude contour lines for $\mu^{+} \tau^{-}$channel at $500 \mathrm{GeV} . \ldots \ldots 7$

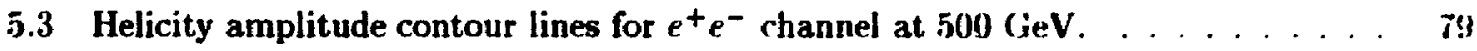

5.4 Differential cross section with respect to $P_{T}$ for the $\mu^{+} \tau^{-}$process at $\mid$'I $+V . \ldots$. .

5.5 Percent contributions of $(-++-)$ helicity amplitude for the $\mu^{+} \tau^{-}$proresss at $50(1)$

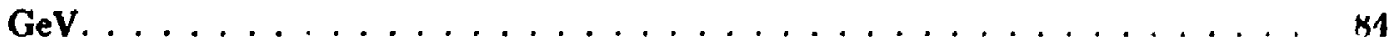

5.6 Percentage contributions of $(-++-)$ amplitude superimposed on $2 \sigma$ contcur for

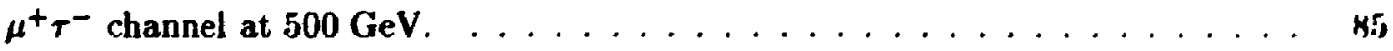

6.1 Cross-section as function of beam energy for the $e^{+} e^{-} \rightarrow W^{+} W^{-}$process. . . . . xx 
6.2 Cross-section as function of energy for $\mu^{+} \tau^{-}$process with varions choices for formfactor parameters $\Lambda$ and $n . \ldots \ldots \ldots \ldots$. . . . . . . . .

7.1 Differential cross-section with respect to $\cos \theta_{7}$ - for the two non-standard cases of CP-even $\kappa_{,}=\kappa_{Z}=0.9$ and CP-odd $\tilde{\kappa}_{Z}=1.0$ for the $\mu^{+} \tau^{-}$channel at $500 \mathrm{GeV} .104$

7.2 Differential cross-section with respect to $M_{\mu \tau}$ for the two non-standard cases of CP-even $\kappa_{\gamma}=\kappa_{z}=0.9$ and CP-odd $\bar{\kappa}_{z}=1.0$ for the $\mu^{+} \tau^{-}$channel at $500 \mathrm{GeV} .104$

7.3 Distributions in $\chi(S)$ for the $\mu^{+} \tau^{-}$process at $500 \mathrm{GeV}$ with $\bar{\kappa}_{Z}=1.0 \ldots \ldots . .112$

7.4 Distributions in $\chi(\mathrm{Q})$ for the $e^{+} e^{-}$process at $1000 \mathrm{GeV}$ with SM couplings. . . 113

B.1 9 diagrams that contribute to the $\mu^{+} \tau^{-}$final state $\ldots \ldots \ldots \ldots$

B.2 9 diagrams extra to those of the $\mu^{+} \tau^{-}$channel that contribute to the $\mu^{+} e^{-}$final state . . . . . . . . . . . . . . . . . . . 119

B.3 19 diagrams extra to those of the $\mu^{+} \tau-$ channal that contribute to the $\mu^{+} \mu^{-}$final state . . . . . . . . . . . . . . . . . 120

B.4 19 diagrams extra to those of the $\mu^{+} \tau^{-}, \mu^{+} e^{-}$, and $\mu^{+} \mu^{-}$channels that contribute to the $e^{t} e^{-}$final state . . . . . . . . . . . . . . . 121

E.1 Schematic Diagram of Four Lepton Production Process $\ldots \ldots \ldots \ldots$ 
This thesis is organized as follows: Chapter 1 contains an introduction to gauge theories and spontaneous symmetry breaking, and describes how these two concepts give rise to the $S C^{\prime}(2)_{L} \times U(1)$ electroweak theory, and how the properties of the $W$ bosons. specitically their couplings to $\gamma$ and $Z$, are uniquely prescribed by the gauge group structure. The most general Lorentz and $U(1)$ gauge invariant triple vertex (of which the $S M$ is a special (ass), is introctuced in Chapter 2; as well, the previous research on the subject of possible anomalous triple boson couplings is summarized there. We consider the processes $e^{+} e^{-}+l^{-}-\overline{\nu l} l^{\prime} \nu$, with all possible lepten configurations, as an indicator of anomalous contributions to the triple boson couplings. 'The' details of the calculation are described in Chapter 3, with a discussion of the helicity amplitudici that contribute to each of the four processes. In Chapter 4, the limits on the coupling paraineters $\kappa_{\gamma}$ and $\kappa_{z}$ from total cross-section measurements are determined. Also in this ('hapter, we discuss how the bounds on the $\kappa_{V}$ parameters might be improved, as well as the possibiblity of discriminating between the effects of the $\gamma$ and $Z$ vertices, through appropriate experimental cuts. In Chapter 5, the possibilities for more tightly constraining the couplings, as well as an alternative means of discriminating betweer: $W W \gamma$ and $W W Z$ effects at a polarized $e^{+} p-$ farility are examined. In Chapter 6 , the effect of introducing unitarity respecting form factors for the $n v$ is investigated. The limits on a CP-violating contribution to the triple vertices are determined in Chapier 7; also, the asym.metries in event rates such a CP-violating coupling would produro. are explored here. Chapter 8 summarizes the results and their possible significance. 


\section{Chapter 1}

\section{Introduction to Electroweak}

\section{Interactions}

In this Chapter, the $S U(2)_{L} \times U(1)$ electroweak theory will be briefly introduced. The historical developmert of the theory is discussed in Section 1.1, with the key concepts of gauge symmetry, the $S U(2)_{L} \times U(1)$ symmetry, and Spontaneous Symmetry Breaking reviewed in Sections 1.2 , 1.3, and 1.4, respectively. In Section 1.5, we give a brief overview of how the electroweak sector of the SM arises from these concepts, and discuss in Section 1.6 the concerns that suggest that the theory is not complete.

\subsection{Historical Development of Electroweak Theory}

The first attempts at a phenomenology of weak interactions were motivated by analogy with the vector currents of the very successful Quantum Electrodynamics (QED). Fermi proposed a model [1] of the weak force involving an effective four-fermion point-interaction. For instance, his model explained beta decay as a process analogous to an electromagnetic transition, but with an electron-neutrino pair playing the role of the emitted photon. The transition from neutron to proton and the emission of the $e-\nu$ pair was assumed to occur at a single point in space-time.

Lee and Yang [2] proposed that invariance with respect to space inversion (parity) might not be respected in the weak interactions. The discovery of parity violation through observation of the fore-aft asymmetry in $\beta$ emission by $W u$ et al. [3] confirmed their ideas. To account for the 
parity violation. Feynman and Gell-Mann [4] and Sudarshan and Marshak [5] modified Vermi's theory. The parity violation was implemented through the introduction of the term $(1-96)$ ints the weak Lagrangian, (the matrix $\gamma_{5}$ is constructed such that the above term projerts out only the left-handed component of a fermion spinor it acts on). The Feynman-( iell-Mams scheme provided an excellent phenomenological account of the observed charged weak interactions. However. it suffered from the same problems as the original Fermi theory, namely, violation of unitarity and non-renormalizability.

The successes of QED are due to the introduction of the concept of a mediating gauge field, the photon. The phaton, through its exchange between charged particles, is believed to comprnsatir for localized phase (or gauge) transformations of the matter fields such that the symmetry of the Lagrangian under such transformations is not lost. This "gauge principle" , that the interations between the fundamental particles are a consequence of maintaining certain symmetries in the universe, is key to the success of QED. Motivated by the success of the gauge principle in QED, we might attempt to construct an analagous model for the weak interaction. However, if we were to build on the existing structure of Feynman's modified four-point theory, the role of the "gauge particle" would be filled by the electron- neutrino pair. To avoid such a situation, the Intermediate Vector Boson (IVB) model [6] was developed. In this model, although not a trur gauge theory (the interactions are not a consequence of maintaining a weak symmetry), the' weak interartions are supposed to be mediated by IVB's, just as electromagnetic ones are tyy $\gamma$ exchange. The observed short range of the weak interaction required the postulated IVB's to br" quite massive; charge conservation could only be satisfied if they carried electromagnetic: charge", they are thus denoted by $W^{ \pm}$.

The 'VB was still both unrenormalizable and violated unitarity for some processses. Thess' problems arose because, although the IVB model was created in analogy with the gauge theory of QED, it was not a gauge theory. The $W^{ \pm}$are not introduced to ensure gauge invariance of the weak Lagrangian, but we:e inserted "by hand". The observed short range of the weak force and strong experimental lower limits on the masses of the $W^{ \pm}$prevent us from imr!ediately "gauging" the theory and having the $W^{ \pm}$play the role of the mediators. This is because the cunanta of an unbroken gauge theory must be massless, in direct contrast with the massive mediators suggested by the short ranged weak force. It was the concept of Spontaneous Symmetry Breakisug(SSBB), developed from the work of Anderson [7], Higgs [8], and Englert, Brout [9], that provided a means for obtaining masses for the IVB's while retaining senormalizability and unitarity of the mestel.

Glashow, Salam, and Weinberg [10] were able to incorporate SSB into a single gauge throry 
which accounted for both the weak interactions and electromagnetism; this unification of two apparently disparate forces became known as the electroweak theory. The observed transitions of the weak force suggested that the matter particles arranged themselv es into doublets and singlets under the weak interaction. The simplest possibility for "gauging" the weak interactions was therefore to assume that the gauge symmetry responsible was a weak $\mathrm{SU}(2)_{L}$ group called "weak isospin", (the subscript " $L$ " indicates that only the left-handed parts of the matier wavefunctions enter into these weak interactions). If we require this $\mathrm{SU}(2)_{L}$ to be a local gauge invariance, we introduce three gauge fields, two with charge \pm 1 to be associated with the $W^{ \pm}$and one electrically neutral, the $W^{0}$. If we were to associate this $W^{0}$ with the mediator of the neutral current processes, then the neutral currents would have the same pure V-A structure as the charged currents; the neutral currents are however not pure $\mathrm{V}$-A. The $\mathrm{SU}(2)_{L}$ group is therefore insufficient on its own to adequately describe the full range of weak interactions. We can however do so, as well as unifying the weak and electromagnetic interactions, by enlarging the $S U(2)_{L}$ group structure to include an Abelian U(1) group called "weak hypercharge" (associated with quantum number $\mathrm{Y}$ ), thus forming the $S U(2)_{L} \times U(1)_{Y}$ electroweak group. Associated with the generators of the $S U(2)$ and $U(1)$ groups were, respectively, three gauge fields $W^{i \mu}(i=1,2,3)$ and a single $B^{\mu}$.

The observed physical mediators of the weak and electromagnetic sectors were then assumed to be suitable combinations of the unphysical $S U(2)_{L} \times U(1)_{Y}$ gauge fields. The charged vector bosons $W^{ \pm}$appear as before, but now with their masses provided in a gauge invariance respecting manner. A massless neutral vector boson appears and is associated with the photon $(\gamma)$ but also there arises a weakly interacting massive neutral vector boson, the $Z$. In a series of papers, ' $t$ Hooft proved both the renormalizability and unitarity of the new theory [11]. What follows is a brief summary of the gauge principle and its inplementation in the electroweak sector.

\subsection{Symmetry and Gauge Theories}

Gauge invariance is the popular candidate for the physical principle which governs the interactions between the elementary particles. The essential concept is that conservation of some charge in a dynamical system implies the existence of a symmetry for the system's Lagrangian, and vice-verss. For instance, the conservation of electric charge is related to the invariance of the QED Lagrangian under global phase transformations of the type $\psi \rightarrow e^{i Q 0} \psi$ for fields $\psi$ with charge $Q$. The corverse is also true: if the Lagrangian is invariant under some continuous trans- 
formation then there will exist a conserved charge associated with this invariance. The above phase transformation is described as global because the $t$ field is transformed in the same manner for all space-time points, ie., the phase parameter $\theta$ is space-time independent. If wo consider the more general case and allow the parameters to be space-time dependent, $\theta \rightarrow \theta(x)$, then to preserve the Lagrangian's invariance or symmetry, we must introduce additional fields. ('onsider the Dirac Lagrangian for a free electron field $\psi(x)$ :

$$
\mathcal{L}=\bar{\psi}\left(i \gamma^{\mu} \partial_{\mu}-m\right) \psi
$$

The Lagrangian Eq. (1.1) is invariant under the transformation

$$
\psi(x) \rightarrow \exp [\operatorname{ie} \theta(x)] \psi(x)
$$

only if we also introduce the field $A_{\mu}(x)$ through the generalized or "covariant" derivative

$$
\partial_{\mu} \rightarrow D_{\mu}=\partial_{\mu}-i e A_{\mu}
$$

where $A_{\mu}(x)$ transforms as

$$
A_{\mu}(x) \rightarrow A_{\mu}+\partial \theta(x) / \partial x_{\mu}
$$

The field $A_{\mu}$ has just the transformation properties required such that it can compensate for the transformed fermion fields; the revised Lagrangian including the $A_{\mu}$ field is

$$
\mathcal{L}=\bar{\psi}\left(i \gamma^{\mu} \partial_{\mu}-m\right) \psi+e \bar{\psi} \gamma^{\mu} A_{\mu} \psi-\frac{1}{4} F_{\mu \nu} F^{\mu \nu}
$$

and this Lagrangian is invariant under the combined transformations of Eq. (1.4) and Eq. (1.3). A mass term for the field $A_{\mu}$ such as

$$
m^{2} A_{\mu} A^{\mu}
$$

explicitly violates the invariance of the Lagrangian of Eq. (1.5). The field $A_{\mu}$ is therefore massless and, if we associate the constant $e$ in Eq. (1.3) with the fermionic electric charge, a reasonable assumption is to associate the field $A_{\mu}$ with the photon. The second term in the Lagrangian Eq. (1.5) is then seen to represent the interaction between the fermion field and the photon, with coupling strength $\mathrm{e}$. The third term is the kinetic energy term of the photon field.

The interaction between any charged particle and the photon is therefore seen as a consequence 
of requiring the Lagrangian to be invariant under the group of local U(1) charge transformations. Similarly gauging the weak isospin and weak hypercharge with their appropriate transformation groups will determine the interactions of the unified electroweak theory.

\section{$1.3 S U(2)_{L} \times U(1)_{Y}$ as a local symmetry}

The observed weak transitions motivate the pairing of the left-handed electron and neutrino together, they form a doublet under the weak isospin $S U(2)_{L}$ group. The right-handed electron stands alone as a singlet under this same group. We therefore define $\chi_{L}$

$$
\chi_{L}=\left(\begin{array}{l}
\nu_{L} \\
e_{L}
\end{array}\right)
$$

and so the free Lagrangian for the electron and its neutrino can be written as

$$
\mathcal{L}=\bar{x}_{L}\left(i \gamma^{\mu} \partial_{\mu}\right) \chi L+\bar{e}_{R}\left(i \gamma^{\mu} \partial_{\mu}\right) e_{R}
$$

To ensure that the abnve Lagrangian remains unchanged under the action of $S U(2)_{L} \times U(1)_{Y}$ at various space-time points, we must introduce four compensating gauge fields. Three of the four, the $W^{i \mu}(i=1,2,3)$, are associated with the generators of the $S U(2)_{L}$ group and the fourth, $B^{\mu}$, with the generator of $U(1)_{Y}$. Just as the gauge field of QED, the photon, is said to couple to electric charge, the $W^{i \mu}$ and $B^{\mu}$ are said to couple to weak isospin and weak hypercharge respectively.

The gauge fields are introduced into the Lagrangian through the covariant derivative $D^{\mu}$ via the replacement:

$$
\partial^{\mu} \rightarrow D^{\mu}=\partial^{\mu}+i g \vec{t} \cdot \vec{W}^{\mu}+i g^{\prime} \frac{Y}{2} B^{\mu}
$$

The gauge fields $W^{i \mu}$ and $B^{\mu}$ must transform under $S U(2)_{L} \times U(1)_{Y}$ in the following manner

$$
\begin{gathered}
B^{\mu} \rightarrow B^{\mu}-\frac{1}{g^{\prime}} \partial^{\mu} \theta(x) \\
W_{i}^{\mu} \rightarrow W_{i}^{\mu}-\frac{1}{g} \partial^{\mu} \theta_{i}(x)-\epsilon_{i j k} \theta_{j}(x) W_{k}^{\mu}
\end{gathered}
$$


The Lagrangian becomes

$$
\mathcal{C}=\bar{\gamma}_{L} \gamma^{\mu}\left(i \partial_{\mu}+g \bar{t} \cdot \vec{W}_{\mu}+g^{\prime} \frac{Y}{2} B_{\mu}\right) \gamma L+\bar{e}_{R} \gamma^{\mu}\left(i \partial_{\mu}+g^{\prime} \frac{Y}{2} B_{\mu}\right) e_{R}-\frac{1}{4} \vec{W}_{\mu \nu} \cdot \vec{i} \vec{i}^{\mu \nu}-\frac{1}{4} H_{\mu \nu} B^{\mu \nu}
$$

where we define the field strength tensors that appear in the kinetic energy terms for the gauge particles as:

$$
\begin{gathered}
W_{\mu \nu}^{i}=\partial_{\mu} W_{\nu}^{i}-\partial_{\nu} W_{\mu}^{i}+g \epsilon^{i j k} W_{j \mu} W_{k \nu} \\
B_{\mu \nu}=\partial_{\mu} B_{\nu}-\partial_{\nu} B_{\mu}
\end{gathered}
$$

The left-handed fields are doublets, so an appropriate representation of the $S U(2)_{L}$ generators are the Pauli matrices $t_{i} \rightarrow r_{i} / 2(i=1,2,3)$. The hypercharge quantum number $Y$ satisfies the relation

$$
Q=T_{3}+\frac{Y}{2}
$$

which indicates how the electromagnetic and weak interactions are linked. Putting $T_{3}= \pm 1 / 2$. and 0 for the doublet and singlet respectively, and inserting charges, the hypercharges are seen to be $Y=-1$ and $Y=-2$ for $X_{L}$ and $e_{R}$, respectively. Thus the Lagrangian becomes

$$
\mathcal{L}=\bar{\chi}_{L} \gamma^{\mu}\left(i \partial_{\mu}-g \frac{\vec{\tau}}{2} \cdot \vec{W}_{\mu}+\frac{g^{\prime}}{2} B_{\mu}\right) \chi_{L}+\bar{e}_{R} \gamma^{\mu}\left(i \partial_{\mu}+g^{\prime} B_{\mu}\right) e_{R}-\frac{1}{4} \vec{W}_{\mu \nu} \cdot \vec{W}^{\mu \nu}-\frac{1}{4} B_{\mu \nu} B^{\mu \nu}
$$

We see that by introducing the gauge fields $W_{\mu}^{i}$ and $B_{\mu}$ to preserve local gauge invariance, we construct terms in the modified Lagrangian which represent interactions between the fermions and these gauge fields. Therefore, interartions between the fermions and the physical $W^{+}, W^{-}, Z$, and $\gamma$, which are composed of combinations of the unphysical $W^{i}$ and $B$, are a consequence of our requirement of local gauge invariance. As given, this Lagrangian predicts that both the elementary fermions and the mediating gauge particles are massless. Inserting mass-terms into this Lagrangian "by hand" would destroy the gauge invariance so carefully constructed. To give the particles masses, and do so in a manner which does not violate the gauge symmetry, we appeal to the mechanism of Spontaneous Symmetry Breaking (SSB) [12]. 


\subsection{Spontaneous Symmetry Breaking}

Bufore attempting to implement SSB in the electroweak Lagrangian of Eq. (1.16), we will demonstrate the principle in a simpler scalar field theory. Consider the Lagrangian for a scalar field $\phi$.

$$
\begin{aligned}
\mathcal{L} & =T-V \\
\mathcal{L} & =\frac{1}{2}\left(\partial_{\mu} \phi\right)\left(\partial^{\mu} \phi\right)+\frac{1}{2} \mu^{2} \phi^{2}-\frac{1}{4} \lambda \phi^{4}
\end{aligned}
$$

where $\lambda>0$ to ensure positive energy density and the term in $\lambda$ represents a self-interaction of the $\phi$ field. In the usual scalar field theory, quantisation would yield particles of mass $\mu$, but here $\mu^{2}$ is regarded only as a parameter.

It is apparent that this Lagrangian is invariant under the transformation $\phi \rightarrow-\phi$. We will explore the consequences of breaking this symmetry on the mass of the scalar field (represented by the term $\left.+1 / 2 \mu^{2} \phi^{2}\right)$. We must consider the two cases

$$
\mu^{2}<0 \text { and } \mu^{2}>0
$$

CASE 1: $\quad \mu^{2}<0$

The Lagrangian is simply that of a scalar field $\phi$ with mass $\mu$. We sketch the potential in Figure (1.1). The potential minimum occurs at $\phi=0$. If we take the potential minimum to define the vacuum, then we have a vacuum expectation value (VEV) for CASE 1 of

$$
\langle\phi\rangle_{\circ}=0
$$




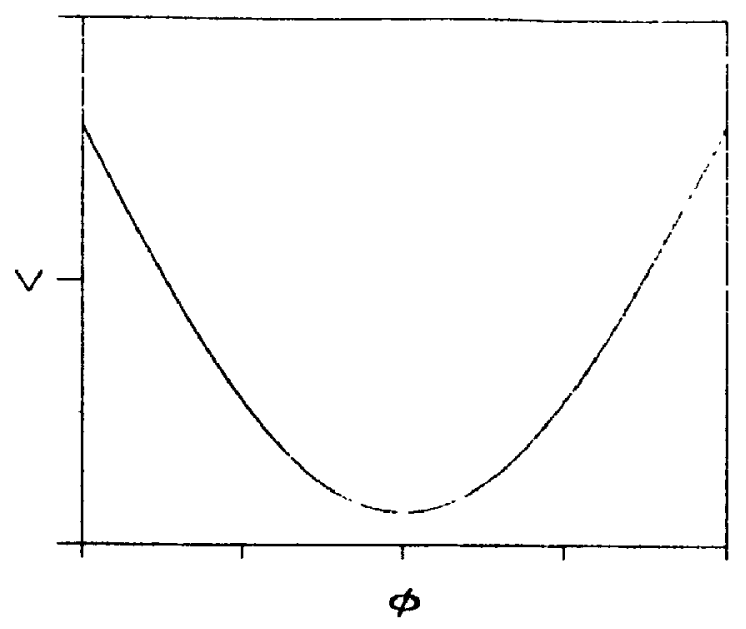

Figure 1.1: Potential as function of $\phi$ for $\mu^{2}>0$

CASE 2: $\mu^{2}>0$

The Lagrangian now has a mass term of the "wrong"sign (the kinetic energy and mass terms should have a relative minus sign). The potential in this case now appears as Figure (1.2).

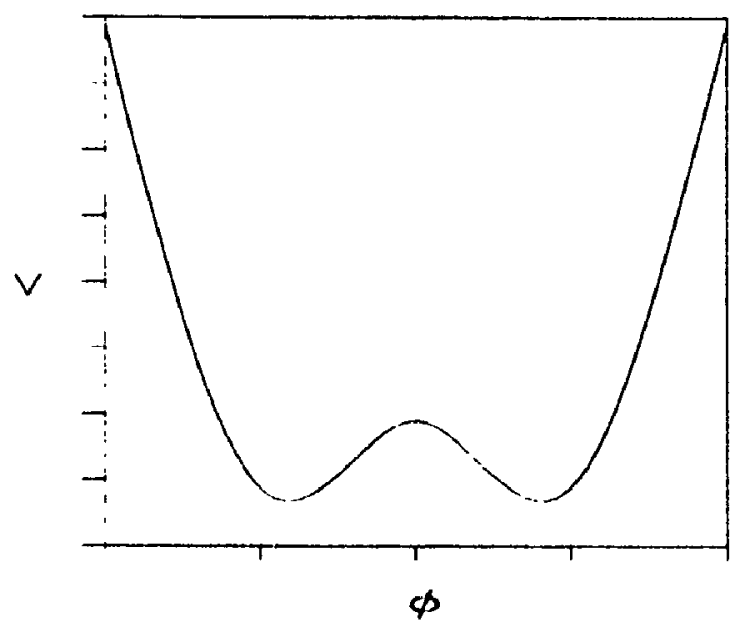

Figure 1.2: Potential as function of $\phi$ for $\mu^{2}<0$

The potential now has 2 minima occurring at

$$
\frac{\partial V}{\partial \phi}=\phi\left(-\mu^{2}+\lambda \phi^{2}\right)=0
$$


solving, we get for these minima

$$
\phi= \pm \sqrt{\frac{\mu^{2}}{\lambda}}
$$

Using the previous definition of the vacuum as occurring at the potential minimum, the field $\phi$ now has a non-zero vacuum expectation value defined as:

$$
\langle\phi\rangle_{o}= \pm v
$$

If we require our vacuum to choose one of the two expectation values, then the syinmetry of the Lagrangian $\phi \rightarrow-\phi$ is lost, or "spontaneously broken". It should be noted that we have not made any changes to the original symmetrical Lagrangian, but merely allowed for the possibility of the vacuum state of the system not explicitly exhibiting this synmetry.

We can define a shifted field $\phi^{\prime} \equiv \phi-v$. With this transformation, we recover a zero VEV for the shifted field.

$$
\left\langle\phi^{\prime}\right\rangle_{0}=0
$$

Making the substitution $\phi=v+\phi^{\prime}$ into the Lagrangian Eq. (1.17), we obtain:

$$
\mathcal{L}^{\prime}\left(\phi^{\prime}\right)=\frac{1}{2}\left(\partial_{\mu} \phi^{\prime}\right)\left(\partial^{\mu} \phi^{\prime}\right)-\lambda v^{2} \phi^{2}-\lambda v \phi^{\prime 3}-\frac{1}{4} \lambda \phi^{\prime 4}
$$

The field $\phi^{\prime}$ has a mass term of the correct sign and a value for the mass of $m=\sqrt{2 \lambda v^{2}}$. The presence of the cubic term means that this transformed Lagrangian does not exhibit the reflection symmetry of the original Lagrangian. It seems that, with such a "wrong-sign" mass term, we must choose between having a symmetrical Lagrangian and a non-symmetrical vacuum state, or vice-versa. We say that the reflection symmetry of the original Lagrangian is spontaneously broken because it is not respected by the vacuum.

It was choosing one of the two possible vacuum states that destroyed the symmetry. It turns out that whenever a system has a degenerate set of possible vacua, we (or nature) must make a choice, and this will destroy the invariance. The example just considered was for the simple case of reflection symmetry but the principle is the same for more complicated continuous symmetries. However, when we spontaneously break a continuous symmetry, there will be massless scalar fields introduced as a by-product of the breaking mechanism. These so called Goldstone bosons appear due to Goldstone's Theorem, which states that a massless scalar field will appear for every continuous symmetry broken. These undesirable (in that thete are no physical massless 
scalars unaccounted forj Goldstone bosons will eventually provide the means for giving the gauge bosons of the electroweak theory their masses when we break the continuous sI ${ }^{\prime}(2)_{f} \times l^{\prime}(1)$ y symmetry. The Goldstones introduced into the theory through the addition of an SSH or Higgs Lagrangian will conspire with the masisless gauge bosons to produce matsive gatuge particles, ats required.

\subsection{Spontaneous Breaking of $S U^{\top}(2)_{L} \times L^{\top}(1)_{Y}$}

We introduce a complex scalar field $\phi$ as before, but now propose that it transform as a douldet under the electroweak symmetry group of $S U(2)_{L} \times U(1)_{Y}$. Therefore, we write this so-c alliul Higgs field as

$$
\phi=\left(\begin{array}{c}
\phi^{+} \\
\phi^{0}
\end{array}\right)
$$

The Higgs Lagrangian is therefore

$$
\mathcal{L}_{H i g g s}=\left(D_{\mu} \phi\right)^{\dagger}\left(D^{\mu} \phi\right)+\mu^{2}\left(\phi^{\dagger} \phi\right)-\lambda\left(\phi^{\dagger} \phi\right)^{2}+\text { gauge kinetic energy terms }
$$

Since our goal is to include this Higgs Lagrangian as part of the locally gauged rertroweak Lagrangian, Eq. (1.16), we introduce the covariant derivative for the Higgs field $D_{\mu}$

$$
D_{\mu} \phi=\left(\partial_{\mu}-i g \frac{\vec{r}}{2} \cdot \vec{W}_{\mu}-i \frac{g^{\prime}}{2} B_{\mu}\right) \phi
$$

We have used the fact that the Pauli matrices are the $S U(2)_{L}$ doublet generators and the hypurcharge of the Higgs field of Eq. (1.19), as defined, is $Y=1$.

If we have the $\mu^{2}>0 \mathrm{SSB}$ case, then we have seen that the physical varuum need not exhibit. the full $S U(2)_{L} \times U(1)_{Y}$ symmetry of the original theory. However, since we require that thr. physical vacuum have zero charge, the vacuum must still respect the $U(1)$ of electromagnetisin Therefore, we must break the full $S U(2)_{L} \times U(1)_{Y}$ down to $U(1)_{E M}$.

The breaking is accomplished by choosing a physical vacuum from the continuum of prosssbilities. As in the simple reflection symmetry example, the SSB case is typified by a non-z/ru 
varuum expectation value for the neutral component of the Higgs field.

$$
\begin{aligned}
& \{\phi\rangle_{n}=\left(\begin{array}{c}
0 \\
\frac{v}{\sqrt{2}}
\end{array}\right) \\
& \text { where } v^{2}=\frac{\mu^{2}}{\lambda}
\end{aligned}
$$

We define a shifted field $\phi^{\prime}$

$$
\phi^{\prime}=\phi-<\phi>\text {, }
$$

We can write the field $\phi^{\prime}$ as

$$
\phi^{\prime}=\frac{1}{\sqrt{2}}\left(\begin{array}{c}
\zeta^{1}+i \zeta^{2} \\
\eta+i \zeta^{3}
\end{array}\right)
$$

and therefore

$$
\phi=\frac{1}{\sqrt{2}}\left(\begin{array}{c}
\zeta^{1}+i \zeta^{2} \\
\eta+v+i \zeta^{3}
\end{array}\right)
$$

which can be rewritten as

$$
\phi=e^{i \frac{.2}{v}}\left(\begin{array}{c}
0 \\
\frac{v+\eta}{\sqrt{2}}
\end{array}\right)
$$

The last form is highly indicative of a gauge transformation on the field $\phi$. We are free to perform another transformation

$$
\phi \rightarrow \phi^{\prime}=e^{-i \frac{r \varepsilon}{*} \phi}
$$

thus

$$
\phi^{\prime}=\left(\begin{array}{c}
0 \\
\frac{n+y}{\sqrt{2}}
\end{array}\right)
$$

Making the above substitution in the Higgs Lagrangian, the total spontaneously broken electroweak Lagrangian is $\mathcal{C}_{H i g g s}$ added to the previous massless gauge boson Lagrangian Eq. (1.16), 
giving the full electroweak Lagrangian

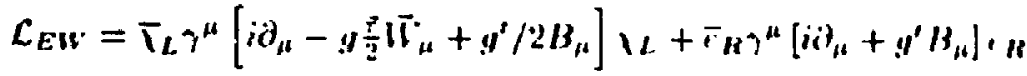

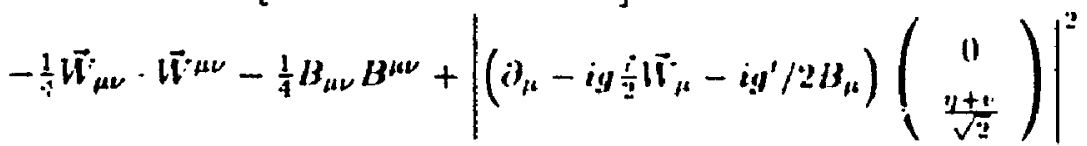

$$
\begin{aligned}
& -\mu^{2} \eta^{2}-\lambda\left(\eta^{3}-\lambda \eta^{3} / 1\right.
\end{aligned}
$$

where $g$ and $g^{\prime}$ are the coupling constants for the two symmetry groups $S\left(t^{\prime}(2)_{l}\right.$, and $/(1)$ introduced in Eq. (1.9).

The particle content and the couplings can now be read off from this Langrangiatl. (If the fonr scalar fields introduced through the SSB process, only the Higgs field $\eta$ remains, with a misss of $m_{H}=\sqrt{2 \mu^{2}}$. Because $\mu$ was a free parameter of the SSB potential, we are unible Io pridict, lih. mass of the Higgs particle. The Higgs has so far escaped detertion, the curront lower limit on the mass is $58.4 \mathrm{GeV}$ [13].

The other three introduced fields, $\zeta^{-1}, \zeta^{-2}$, and $\zeta^{-3}$, which are the previously mentioned cioldstone bosons, were gauged away; see Eq. (1.27). We know that such a gauging requircs a comprinsating transformation of the gauge boson fields, see Eqs. (1.10,1.11). The uature of 1:|s. (1.27) and $(1.10,1.11)$ means that the transformation of the gauge fields will involve the (ioldistuncs, and it is these new transformed fields that are given masses. The (ioldstones, through the gange transformation, provide the longitudinal polarization necessary for a massive vertor field. "The massless gauge fields are said to "eat" the massless Goldstones to become massiver.

The masses of the physical gauge bosons (taken as the following combinations of the $W^{*}$ ind B.)

$$
\begin{gathered}
W^{ \pm}=\frac{1}{\sqrt{2}}\left(W^{1} \mp i W^{2}\right) \\
Z=W^{3} \cos \theta_{W}-B \sin \theta_{W} \\
A=W^{3} \sin \theta_{W}+B \cos \theta_{W}
\end{gathered}
$$

are found to be

$$
\begin{gathered}
M_{w}=\frac{v g}{2} \\
M_{Z}=\frac{v}{2} \sqrt{g^{2}+g^{\prime 2}} \\
M_{\gamma}=0
\end{gathered}
$$


with the electroweak mixing angle $\theta_{W}$ defined through the relations

$$
\begin{aligned}
& \cos \theta_{W}=\frac{g}{\sqrt{g^{2}+g^{\prime 2}}} \\
& \sin \theta_{W}=\frac{g^{\prime}}{\sqrt{g^{2}+g^{\prime 2}}}
\end{aligned}
$$

With the above substitutions for the physical gauge bosons made into the electroweak Lagrangian, the Feynman rule for the couplings between two W's and either a $\gamma$ or $Z$ can be read off from Eq. (1.29). With the momenta of the incoming $V, W^{+}$, and $W^{-}$defined as $p, k$, and $q$ respectively, the Feynman rule for the trilinear couplings, as shown in Figure (1.3), is as follows:

$$
\Gamma_{\mu \nu \lambda}=i g_{V}\left[g_{\nu \lambda}(p-q)_{\mu}+g_{\lambda \mu}(q-k)_{\nu}+g_{\mu \nu}(k-p)_{\lambda}\right]
$$

where $g_{V}$ equals $e(\gamma)$ or $e \cot \theta_{W}(Z)$.

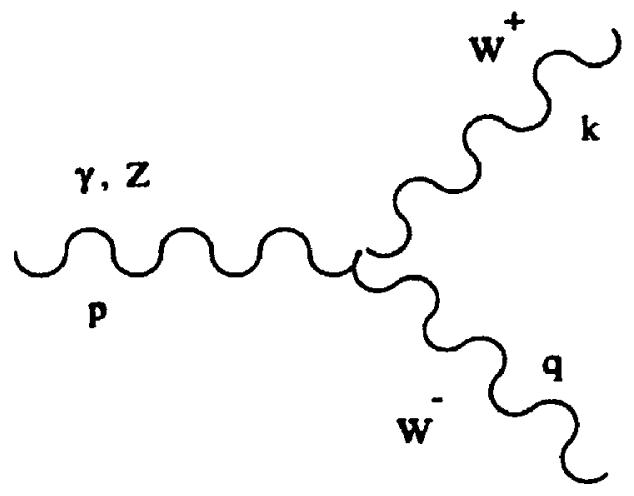

Figure 1.3: 'Triple Gauge Boson Vertex

\subsection{Problems with the Standard Model}

If we combine the electroweak group of $S U(2)_{L} \times U(1)_{Y}$ with the symmetry group of the strong interaction, $S U(3)$, then the result is known as the Standard Model [SM]. The SM has been extremely successful in both explaining observed phenomena and in predicting new physics. The 
fact that the SM describes the basic forces through the local gauge invariance principle ensures that the infinities that occur in higher order calculations can be handled in a consistent manner. Despite its successes, the SM is not presently believed to be the ultimate theory of the particle interactions. There remain both real and philosophical problems with the present SM framewurk. These problems include the following

1. It does not explain the repetition of fermion families

(the $\mu$ and $\tau$ appear extra to the electron)

2. Model has too many free parameters

(6 lepton masses, 6 quark masses, 4 lepton mass mixing angles, 4 quark mass mixing angles, 3 electroweak parameters, 1 Higgs mass, $1 \mathrm{CP}$ violating phase, and 1 QCD coupling constant)

3. Does not incorporate gravity

(a quantum description of gravity, a necessary step in its unification with the SM, is presently missing)

4. The question of $\mathrm{CP}$ violation

(in the SM, CP violation is introduced through a complex phase in the CKM matrix, there are however other possibilities)

5. Why are weak interactions different from those of electromagnetism?

(why are the $W$ 's and $Z$ so massive)

We are therefore mot:vated to consider the possibility that the SM will turn out to be only a low energy limit of a deeper, more fundamental theory. There are essentially four main theoretical ideas for physics beyond the SM; supersymmetry, technicolour, extended gauge theories, and composite models. Each of these extensions to the SM predict a wide range of new particles and phenomena. The challenge facing physicists is to discover evidence of this possible new physics and, if detected, to determine its origin. To probe for new physics we haye a number of experimental approaches to choose from; we can go to higher and higher energies in the hope that there will be sufficient energy to produce the exotic particles, we can search for processes which are forbidden in the Standard Model, or we can compare the results of precision measurements to their SM predictions.

In the last approach, we can parametrize any deviation of a precision measurement from the SM prediction by allowing the Feynman rules for the couplings of the elementary particles to 
vary from their SM values. New physics, as indicated by disagreement between measurement and prediction, would then be evidence for deviations of these couplings from their SM expressions. By modelling the sensitivity of physical observables such as total and differential cross-sections to any such variations in the SM couplings, we can determine what processes would be most suitable for searching for such new physics.

As stated before, the electroweak $S U(2)_{L} \times U^{\prime}(1)_{Y}$ model has been confirmed by all experiments up to now, no significant discrepancy between precision measurement and SM prediction has yet been discovered [14]. This empirical evidence has been almost exclusively restricted to boson-fermion interactions. The gauge-boson self-interactions, Eq. (1.32), which are a consequence of the non-abelian natura of the electroweak gauge group, are essential to both the renormalizability and unitarity of the theory and so are key aspects of the SM. These boson self-couplings are precisely predicted by the SM and their measurement will be an important test of the theory.

Future colliders like LEP 11 ( $e^{+} e^{-}$at $\left.\sqrt{s} \simeq 200 \mathrm{GeV}\right)[15,16]$ and the proposed Next Linear Collider (NLC) $\left(e^{+} e^{-}\right.$at $\left.\sqrt{s}=500 \mathrm{GeV}\right)[17,18,19]$ will make possible direct tests of the gauge boson self interactions through the examination of processes which get tree level contributions from these self-couplings. The measured observables of such processes will therefore be indicators of new physics in the gauge self-interactions; a discrepancy between an experimental cross-section and the SM prediction can be interpreted as evidence of a non-standard coupling in the triple boson vertices.

In this thesis, the sensitivity of the four lepton production processes $e^{+} e^{-} \rightarrow l^{+} \nu l^{\prime-} \bar{\nu}$ to deviations of the triple boson vertices from their Standard Model axpressions will be explored. The more sensitive are the physical observables of the processes to such deviations, the smaller are the deviations that would be measurable, and the better the discovery potential of the process. As a realistic experiment will have limited measurement precision due to statistics and systematics, a measurement of an observable such as a total cross-section, with its error, will not uniquely determine the 3-boson couplings, but will determine a range of passibilities. The measure of the discovery potential will therefore be how stringent are the bounds on this range, ie. how tightly we can constrain any such deviation. 


\section{Chapter 2}

\section{Theoretical Background}

In this Chapter, I will discuss the theoretical background for the calculation. In Section 2.1 a general parametrization of the Triple Boson Vertices and theoretical estimates of possible nonstandard contributions are listed. A summary of the previous research is given in Sertion 2.2, Section 2.3 is a discussion of our motivation for choosing the four lepton production processes over other possibilities.

\subsection{Triple Boson Vertex}

The Feynman rule for the triple boson vertices, Eq. (1.32), was derived from the bosonic sector of the Standard Model Lagrangian, Eq. (1.29). The portion of Eq. (1.29) which determines the Feynman rules for the $\gamma \mathrm{WW}$ and $\mathrm{ZWW}$ vertices has its structure uniquely determined by the symmetry requirements of the Lagrangian under $S U(2)_{L} \times U(1)_{Y}$. We could also conjecture a more general Lagrangian that respects only more basic symmetries such as Lorentz invariance and $U(1)_{E M}$ gauge invariance; the SM would then be a special case of this more general Lagrangian. An effective Lagrangian, that respects gauge and Lorentz invariance, for the triple boson vertices is often parametrized as [20] 


$$
\begin{aligned}
L_{v W W} / g_{V W W} & =i g_{1}^{V}\left(W_{\mu \nu}^{\dagger} W^{\mu} V^{\nu}-W_{\mu}^{\dagger} V_{\nu} W^{\mu \nu}\right)+i \kappa v W_{\mu}^{\dagger} W_{\nu} V^{\mu \nu} \\
& +\frac{i \lambda_{V}}{m_{W}^{2}} W_{\lambda \mu}^{\dagger} W_{\nu}^{\mu} V^{\nu \lambda}-f_{4}^{V} W_{\mu}^{\dagger} W_{\nu}\left(\partial^{\mu} V^{\nu}+\partial^{\nu} V^{\mu}\right) \\
& +f_{5}^{V} \epsilon^{\mu \nu \rho \sigma}\left(W_{\mu}^{\dagger} \bar{\partial}_{\rho} W_{\nu}\right) V_{\sigma}+i \bar{\kappa}_{\nu} W_{\mu}^{\dagger} W_{\nu} \tilde{V}^{\mu \nu} \\
& +\frac{i \bar{\lambda}_{V}}{m_{W}^{2}} W_{\lambda \mu}^{\dagger} W_{\nu}^{\mu} \dot{V}^{\nu \lambda}
\end{aligned}
$$

In the above, $V$ stands for either the photon or $\mathrm{Z}$ field, $W^{\mu}$ is the $W-$ field, $W_{\mu \nu}=\partial_{\mu} W_{\nu}-$ $\partial_{\nu} W_{\mu}, V_{\mu \nu}=\partial_{\mu} V_{\nu}-\lambda_{\nu} V_{\mu}, \check{V}_{\mu \nu}=\frac{1}{2} \epsilon^{\mu \nu \rho \sigma} V_{\rho \sigma}$, and $\left(A \bar{\partial}_{\mu} B\right)=A\left(\partial_{\mu} B\right)-\left(\partial_{\mu} A\right) B$ The overall couplings are $g_{\gamma}=\mathrm{e}$ and $g_{z}=\mathrm{e} \cot \theta$. This effective Lagrangian determines the effective three boson vertex as

$$
\begin{aligned}
\Gamma_{V}^{\alpha \beta \mu}(q, \bar{q}, P) & =\left(g_{1}^{V}+\frac{s}{2 m_{W}^{2}} \lambda_{V}\right)(q-\bar{q})^{\mu} g^{\alpha \beta}-\frac{\lambda_{V}}{m_{W}^{2}}(q-\bar{q})^{\mu} P^{\alpha} P^{\beta} \\
& +\left(g_{1}^{V}+\kappa V+\lambda_{V}\right)\left(P^{\alpha} g^{\mu \beta}-P^{\beta} g^{\mu \alpha}\right)+i f_{4}^{V}\left(P^{\alpha} g^{\mu \beta}+P^{\beta \beta} g^{\mu \alpha}\right)+i f_{5}^{V} \epsilon^{\mu \alpha \beta \rho}(q-\bar{q})_{\rho} \\
& -\bar{\kappa}_{V} \epsilon^{\mu \alpha \beta \rho} P_{\rho}-\frac{\bar{\lambda}_{V}}{m_{W}^{2}}\left(q^{\omega} g_{\alpha}^{\gamma}-q^{\gamma} g_{\alpha}^{\omega}\right)\left(\bar{q}^{\gamma} g_{\beta}^{\rho}-\bar{q}^{\rho} g_{\beta}^{\gamma}\right)\left(P^{\sigma} g_{\mu}^{\tau}-P^{\tau} g_{\mu}^{\sigma}\right) \epsilon_{\alpha W \sigma r}
\end{aligned}
$$

where $\mathrm{P}, \mathbf{q}$, and $\bar{q}$ are the momenta of the incoming neutral vector boson, $W^{+}$, and $W^{-}$, respectively.

The -ze-level Standard Model values of the parameters of Eq. (2.2) are

$$
g_{1}^{V}=\kappa_{V}=1, \quad \lambda_{V}=f_{4}^{V}=f_{5}^{V}=\bar{\kappa}_{V}=\tilde{\lambda}_{V}=0
$$

With these substitutions, the SM expression for the vertex Eq. (1.32) is recovered from Eq. (2.2).

The parameters $\kappa_{\gamma}$ and $\lambda_{\gamma}$ are related to the static magnetic dipole and electric quadrupole moments of the $\mathrm{W}$ boson as follows

$$
\begin{aligned}
\mu_{W} & =\frac{e}{2 M_{W}}\left(1+\kappa_{\gamma}+\lambda_{\gamma}\right) \\
Q_{w} & =-\frac{e}{2 M_{W}}\left(\kappa_{\gamma}-\lambda_{\gamma}\right)
\end{aligned}
$$

Of the seven triple boson vertex parameters, (or form-factors if we allow for energy dependence), $g_{1}^{v}, \kappa_{V}, \lambda_{V}$, and $f_{5}^{v}$ parametrize terms of the effective Lagrangian that respect CP symmetry: $f_{4}^{V}, f_{6}^{V}$, and $\tilde{\lambda}_{V}$, terms that violate CP invariance. Because CP violation is intro- 
duced in the SM through phase effects in the quark-sector CKM matrix, the ('P violating form factors are zero even at one loop in the SM. I'pper bounds on the one loop correttions to the ('P' respecting form factors are at the percent level in the SM. For instance, the limits on $\kappa_{\gamma}$ and $\lambda_{\gamma}$ have been calculated at the one-loop !evel in the SM [21].

$$
\begin{aligned}
& \left(\Delta \boldsymbol{\kappa}_{\gamma}\right)_{\max }=1.5 \% \\
& \left(\Delta \lambda_{\gamma}\right)_{\max }=0.25 \%
\end{aligned}
$$

In extensions of the Standard Model, such as those with extra Higgs or extra heavy fermions [21], or Supersymmetry (SUSY) [22], the expected limits on the deviations of the ('P-rvell couplings from their tree level Standard Model values are the same order of magnitude as these SM loop corrections, $\sim 1 \%$. If however the $\mathbf{W}$ bosons are composite objects, then very large deviations of the form factors from their SM values are possible. For instance, kv has been shown to exceed 3 in one model [23].

The CP-odd couplings can appear at one loop level in extensions to the SM such as those listed above, and Left-Right Symmetry models, but tend to be smaller than the CP-even ones due: to the loop suppression. For instance, in a Left-Right Symmetric model where C:P violation arises in the mixing of the $S U(2)_{L}$ and $S U(2)_{R}$ gauge bosons [24], approximate values at $\sqrt{\kappa}=200$ $\mathrm{GeV}$ are $f_{4}^{Z}=10^{-4}, \tilde{\kappa}_{Z}=10^{-4}$, and $\tilde{\lambda}_{\gamma}=10^{-3}$. In general, $\tilde{\lambda}_{V}$ is the least likely of the CP-odd form factors to be generated at one loop in non-standard models [25].

\subsection{Previous Research}

Various approaches have been proposed for measuring the WWV vertices. Belanger et al. [26] suggested that precision measurements of the $\mathrm{Z}$ boson couplings to fermions could constrain the WWZ vertex. The WWZ vertex contributes via the looo diagram given in Figure (2.1b). 


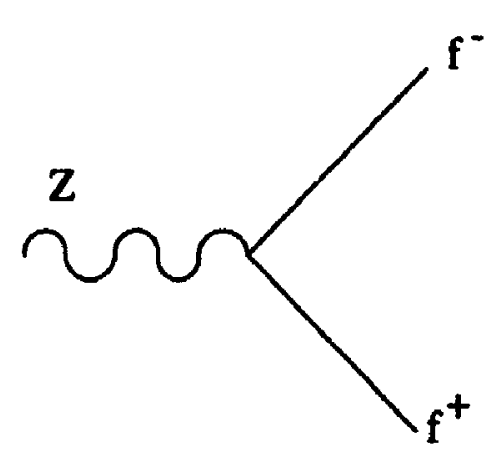

(a)

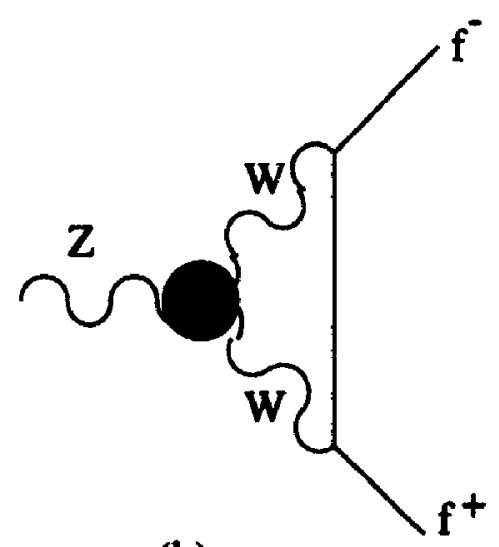

(b)

Figure 2.1: The tree level Feynman diagram for $\mathbf{Z}$ boson coupling to fermions (a), and the loop diagram involving the $Z W W$ vertex (b).

This loop correction results in a modification of the left handed fermion couplings to

$$
C_{L}=I_{3 L}-Q \sin ^{2} \theta_{\omega}+\delta
$$

where

$$
\delta=\frac{\alpha}{8 \pi \sin ^{2} \theta_{\omega}}\left[-\frac{4}{3} g^{2}-1+\lambda Z-\frac{1}{2} \delta \kappa_{z}\right] \log \left(\Lambda^{2} / M_{W}^{2}\right)
$$

We restrict ourselves to the CP-even set of vertex form factors. This shift in $C_{L}$ will result in a change in the partial widths of the $Z^{0}$;

$$
\frac{\Delta \Gamma_{f}}{\Gamma_{f}}=\frac{2 \delta C_{L}^{f}}{\left[\left(C_{L}^{f}\right)^{2}+\left(C_{R}^{f}\right)^{2}\right]}
$$

By comparing the experimental measurements of the partial widths to the above expression, we can constrain the anomalous WWZ couplings. For example, an experimental error on $\Gamma_{b}$ of $1 \%$ gives the constraints $\left|\lambda_{z}\right| \leq 0.34, \quad \delta \kappa_{z} \mid \leq 0.68$. These constraints approach the predicted "interesting" level of precision. However, because it is a loop calculation, the derived limits are indirect.

The prospect of directly measuring the triple boson vertices at future high luminosity, high energy colliders, through production of real gauge bosons, has motivated numerous authors to 
study the subject. Gaemers and Gounaris gave general parametrizations for the couplings of the electroweak bosons [27]. Chen and Renard [28] and also Robinett [29] discussed the general coupling parameters in the context of composite models. Most of these early studies of thr. anomalous couplings were restricted to those parameters that satisfied C,P, and I invariance. separately. Bilchak and Stroughair [30] examined the sensitivity of the angular distributions of the decay leptons from $e^{+} e^{-}$production of W's to non-standard values of $\kappa^{2}$. At LEP2 energies. they found that these couplings could be determined to within approximately $50 \%$ for $\mathrm{n}_{2}$ and $35 \%$ for $\kappa_{Z}$. Much better constraints could be obtained through studies at higher energies, or if the incoming electron and positron were polarized. They also compared the predicted corrections from the one loop contributions, calculated by Lemoine and Veltman [31] and Philippe [32], 10 those from anomalous couplings. The radiative corrections were generally of the opposite sign to the non-standard coupling effects, and showed very different angular behaviour.

Hagiwara et al. [20] performed an extensive analysis of $e^{+} e^{-} \rightarrow W^{+} W^{-}$at LEP2 energies, with a systematic study of observable experimental distributions to determine those most sensitive to the various anomalous couplings. They found that, with a sample of $10^{4} \mathrm{~W}$ 's, they could constrain $\kappa-1$ and $\lambda$ to within $10 \%$. For couplings such as $\kappa$ and $\lambda$, which parametrize effertive Lagrangian terms that respect $\mathrm{CP}$, the most sensitive experimental measure was determined to be the differential angular distribution of the produced W's. For the CP-odd parameters, the most sensitive distributions were those involving combinations of the polar and azimuthal angless of the $\mathbf{W}$ decay products.

To differentiate between the effects of the $\gamma \mathrm{WW}$ and $\mathrm{ZWW}$ vertices, Couture, (iodfrey, and Lewis [33] studied the process $e^{+} e^{-} \rightarrow \mu^{+} \mu^{-} \nu \bar{\nu}$. By cutting on the invariant mass of the $\mu^{+} \mu^{-}$ pair the $Z$ couplings can be determined to $\Delta \kappa_{Z}=0.4$ and $\Delta \lambda_{Z}=0.4$ at $95 \%$ C.L., independent of the $\gamma$ couplings. Grosse-Knetter and Schildknecht [34] studied the vector boson production processes, $e^{+} e^{-} \rightarrow W^{+} W-Z$ and $e^{+} e^{-} \rightarrow W^{+} W^{-} \gamma$ to independently measure $\kappa_{\gamma}$ and $\kappa_{Z}$. They found, at $\sqrt{8}=500 \mathrm{GeV}$, detection limits of

$$
\begin{array}{cc}
e^{+} e^{-} \rightarrow W^{+} W^{-} Z & 0.95<\kappa_{Z}<1.06 \\
e^{+} e^{-} \rightarrow W^{+} W^{-} \gamma & 0.98<\kappa_{\gamma}<1.09
\end{array}
$$

and that these limits could be improved by an order of magnitude if the energy was increassed to $\sqrt{s}=2000 \mathrm{GeV}$. Baur and Zeppenfeld [35] applied partial wave unitarity to the proresws $e^{+} e^{-} \rightarrow W+W-$ to place limits on the possible deviations of the $y W W$ and $Z W W$ verticess from 
their SM predictions. The derived limits depend strongly on the proposed scale at which the new physics becomes operative.

Gounaris, Schildknechht, and Renard [36] examined the sensitivity of the angular distributions of the decay products of $e^{+} e^{-} \rightarrow W^{+} W^{-}$to CP violating contributions in the triple boson vertices. Chang, Keung, and Phillips [37] investigated various charge energy asymmetries and azimuthal asymmetries as tests of CP-odd couplings, once again through study of the process $e^{+} e^{-} \rightarrow W^{+} W^{-}$. More recently, Likhoded et al. [38] studied the role of initial beam polarization in $W$ pair production in improving the bounds obtained from the unpolarized case. They also investigated restricting the allowed range of phase space, in order to optimize sensitivity to the vertex parameters by excluding the dominant non-dependent $t$-channel neutrino exchange contribution.

The processes of associated $W$ production $p \bar{p} \rightarrow W \gamma X ; W \rightarrow l \nu$ and radiative $W$ decay $p \bar{p} \rightarrow W X ; W \rightarrow \gamma l \nu$ have been proposed as means of examining the $W W \gamma$ vertex independently $[39,40,41,42,43,44]$. Associated $W$ production, although very sensitive to $\kappa$, has a relatively small cross-section and a large background from the process $p \bar{p} \rightarrow W$ jet $X$, where the jet is misidentified as a photon. Cortes et al. [43] estimate a measurement of $-1.0<\kappa_{\gamma}<3.0$ might be possible at Tevatron energies. Baur and Zeppenfeld [40], examining the same associated production process but at LHC and SSC energies, predict limits of $0.82<\kappa_{\gamma}<1.18$. The radiative $W$ decay process was consitered by Cortes et al. [43]. This process has a relatively large cross section but has only moderate $\kappa_{\gamma}$ dependence and is expected to give only a $100 \%$ measurement of $\kappa_{\gamma}$.

The possibility of obtaining intense photon beams from beamstrahlung or backscattered laser beams has motivated consideration of the processes $e \gamma \rightarrow \nu W$ and $\gamma \gamma \rightarrow W^{+} W^{-}$as a testing ground for the triple boson couplings $[45,46,47,48,49,50]$. Couture, Godfrey, and Kalyniak [48], studying the er $\rightarrow \nu W$ process, predicted bounds of $0.8<\kappa_{\gamma}<1.2$ with a integrated luminosity of $500 \mathrm{pb}^{-1}$ at $\sqrt{s}=120 \mathrm{GeV}$. Yehudai [49] suggested that bounds of $\sim 5 \%$ are achievable through the same process at a $1 \mathrm{TeV} e^{+} e^{-}$collider. Choi and Schrempp [50], in their analysis of the $\gamma \gamma \rightarrow W^{+} W^{-}$process with laser photons, conclude that significant constraints are possible, $0.98<x_{\gamma}<1.04$.

The photoproduction of a single $W$ at ep colliders has been proposed as a precise test of the $W W \gamma$ vertex. The situation at an ep collider is much cleaner than in $p \bar{p}$ colliders, where $a W$ and a photon have to be identified in the final state. Future measurements of $W \gamma$ at the DESY ep collider HEHA through single $W$ production ep $\rightarrow e W+X[51]$, limited by poor statistics, are 
not expected to give limits better than $50 \%$. Kim $e t$ al. [52] examined the processes ep $\rightarrow W, Z$. taking the ratio of $W$ and $Z$ production cross-sections, and predicted bounds of $0.8<\kappa_{\gamma}<1.2$ for LEP × LHC energies.

There are recent experimental observations of the $\mathrm{WW}_{\gamma}$ couplings reported from measurements of $\sigma\left(p \bar{p} \rightarrow W^{ \pm} \gamma\right)[53,54,55]$ The UA2 collaboration [53] placed 95\% C.L. limits of $-3.5<\kappa_{\gamma}<5.9$. A combined limit from the D0 [54] and CDF [55] collaborations has been given as $-1.3<\kappa_{\gamma}<3.2$. Also, the CDF collaboration has reported relatively stringent $95 \%$ C.L. bounds of $0.1<\kappa_{V}<2.0$ from their measurement of the processes $p \bar{p} \rightarrow W^{+} W^{-}, W^{ \pm} Z, W^{ \pm} \gamma$. Mckellar and He [56], in their analysis of recent CLEO data [57] on $b \rightarrow s \gamma$. obtain bounds on $\kappa_{\gamma}$ of $-1.5<\kappa_{\gamma}<1.44$. While these experimental bounds are compatible with the SM, they are still too weak to be considered a precision test of the theory.

\subsection{Motivation for Studying $e^{+} e^{-} \rightarrow l^{+} \nu l^{\prime-} \bar{\nu}$}

From this summary of previous research, it is apparent that much attention has been focussed on the process $e^{+} e^{-}+W^{+} W^{-}$as a testing ground for the triple boson vertices at an $e^{+} e^{-}$collider. The popularity of the process is due to its relative sensitivity to deviations of the boson couplings from the SM. The enhanced sensitivity reflects the precise cancellation that occurs among the three, individually $\sqrt{S}$-diverging diagrams, Figure (2.2). If the couplings are allowed to deviate from their SM values, this cancellation is destroyed, and consequently the cross-section increases with $\sqrt{8}$. Thus, a small deviation of a coupling parameter from its gauge value can have a large effect on the experimental observables at sufficiently high energies.

Because the W's decay before being directly detected, it is their decay products which are experimentally observed. The momentum and polarizations of the parent $W$ 's must then be reconstructed from the observed decay products. We here concentrate on the purely leptonic $W$ decays. For this case of both $W$ Łosons decaying to a pair of leptons, eg. $e-D$, the undetected neutrinos prevent the unambiguous reconstruction of the $W$ 's. Thus, although it is appropriate to study W production at LEP2 energies, (where the processes are dominated by the nearly on-shell $W$ propagators), at higher energies, we must examine the complete production procews for the purely leptonic case. The difference between the two posible procesues, 


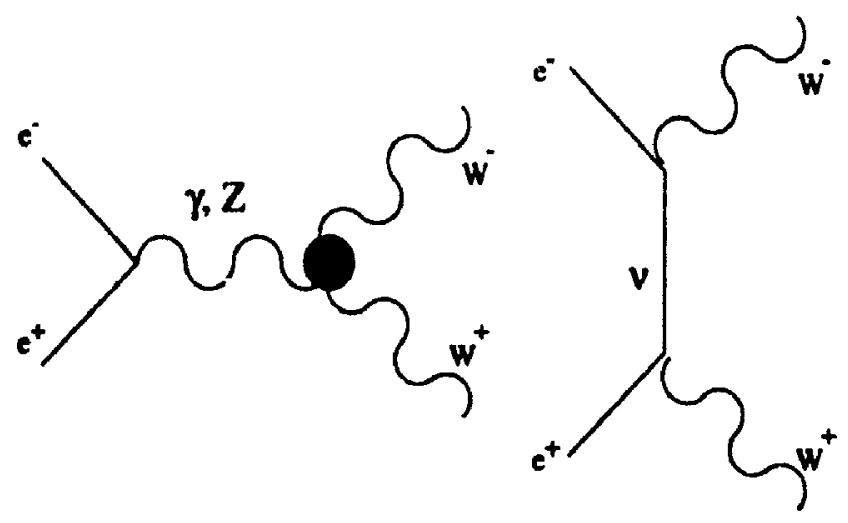

Figure 2.2: Feyman diagrams that contribute to $\mathrm{W}$-pair production. The triple boson vertex is indicated by the shaded circle.

$$
\begin{aligned}
& e^{+} e^{-} \rightarrow W^{+} W^{-} \rightarrow l^{+} \nu l^{-} \bar{\nu} \\
& e^{+} e^{-} \quad \rightarrow \quad l^{+} \nu l^{\prime-} \bar{\nu}
\end{aligned}
$$

becomes substantial as we exceed the $W$ pair threshold. We can more rigorously justify the need to examine the full process, $\mathrm{e}^{+} e^{-} \rightarrow l^{+} \nu l^{-} \bar{\nu}$, ( which has as a subset of its diagrams the process $\left.e^{+} e^{-} \rightarrow W^{+} W^{-} \rightarrow l^{+} \nu l^{-} \bar{\nu}\right)$ by examining the process, $e^{+} e^{-} \rightarrow \mu^{+} \nu e^{-} \bar{\nu}$, at $\sqrt{s}=500 \mathrm{GeV}$, subject to a variety of cuts on the invariant masses of the $\mu^{+} \nu$ and $e^{-\bar{\nu}}$ pairs. Of course, the undetected neutrinos prevent the experimental implementation of such a cut. Nevertheless, by constraining, either singly, or in combination, the lepton pair invariant masses to lie within \pm 5 Gev of the $W$ boson mass, we effectively reduce the full process to single $W$, or $W$ pair production. By requiring both invariant masses to lie in the given range, we exclude the contributions to the full process that are extra to $e^{+} e^{-} \rightarrow W^{+} W^{-} \rightarrow l^{+} \nu l^{\prime-} \bar{\nu}$, and so are effectively left with this $W$ pair production process. For the following cuts:

$$
\begin{array}{lcc}
\text { 1) } M_{W}-5 \mathrm{GeV} & <M_{\mu \nu}< & M_{W}+5 \mathrm{GeV} \\
\text { 2) } M_{W}-5 \mathrm{GeV} & <M_{\bar{e}}< & M_{W}+5 \mathrm{GeV} \\
\text { 3) } M_{W}-5 \mathrm{GeV} & <M_{\bar{e}}, M_{\mu \nu}< & M_{W}+5 G e V
\end{array}
$$


the cross-sections, relative to that of the full process. were found to be in the ratio $1: 0.84: 0.5 .4$ : 0.44 , respectively. Clearly, $W$ production is unable to sufficiently approximate the full four lepton production process, it accounts for less than half of the total cross-section in this example. As well, approximating the complete four lepton production process by $W$ pair production neglents the extra triple boson vertex dependent diagrams involved in the full four lepton production process. The s-channel $\gamma$ and $Z$ diagrams of $W$ pair production are augmented by, depending on the specific final state, t-channel $W$ 's, a $\gamma, Z$ and $W$,or a $W$ and $\gamma, Z$. These "extra" vertex dependent diagrams can produce very different sensitivity to the coupling deviations thall do the $W$-pair s-channel $\gamma$ and $Z$ diagrams. Thus, in order to more realistically examine the sinsitivity to the couplings of the triple boson vertex at the higher energies accessed by future colliders, we must calculate the full $e^{+} e^{-} \rightarrow l^{+} \nu l^{\prime-} \bar{\nu}$ production process. 


\section{Chapter 3}

\section{Four Lepton Production}

\section{Processes}

We describe in this Chapter the types of four lepton production processes, the Feynman diagrams that contribute to the four different final state lepton configurations, Section 3.1 , and the different helicity amplitudes that contribute to the different final state processes, Section 3.2. In Section 3.3 and Appendix $E$ the details of the calculation, including possible backgrounds and cuts, are discussed.

\subsection{Feynman Diagrams}

We consider the processes $e^{+} e^{-}+l^{+} \nu l^{-} \bar{\nu}$ as tests of the triple boson vertices. We examine all possible charged lepton combinations, specifically the final state pairings of $\mu^{+} \tau^{-}, \mu^{+} e^{-}\left(\tau^{+} e^{-}\right)$ . $\mu^{+} \mu^{-}\left(\tau^{+} \tau^{-}\right)$, and $e^{+} e^{-}$. At the high energies of $\sqrt{s} \geq 500 \mathrm{GeV}$ considered, the mass difference between the $\mu$ and the $\tau$ is insignificant, so the channels in parentheses are equivalent to their unparenthesized partners. For the first two channels, $\mu^{+} \tau^{-}$and $\mu^{+} e^{-}$, the charge conjugate processes, $\mu^{-} \boldsymbol{r}^{+}$and $\mu^{-} e^{+}$, serve to double the total cross-section. The complete set of Feynman diagrams that contribute to each of the different four lepton production processes is given in Appendix $B$; we describe here the general classes of contributing diagrams and helicity amplitudes for the different channels.

The $\mu^{+} \tau^{-}$state has 9 different contributing diagrams, two of which involve the triple boson 
vertex and thus show sensitivity to the gWW and $Z W W$ couplings. These two diagrants are the s-channel $\gamma$ and $Z, W$ pair production, with subsequemt W decaly intu $\mu^{+} y$ and $r^{-} \bar{t}$ pairs, their form is illustated in Figure (3.1).

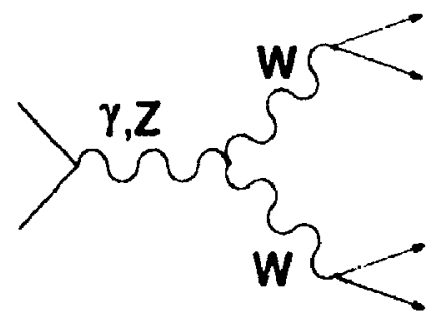

Figure 3.1: Triple boson vertex dependent diagrams that contribute to all possible four lepton final state configurations.

Of the 7 parameter independent diagrams, the dominant contributor is t-chanmel meutrimos exchange, Figure (3.2a), the other 6 are represented in Figure (3.2b). The s-chaunel nerutral boson couples to charged leptons, the s-channel $Z$ couples to either charged leptons or neutrimos. and the $W$ can be produced from either fermion leg.

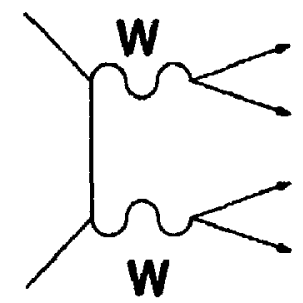

(a)

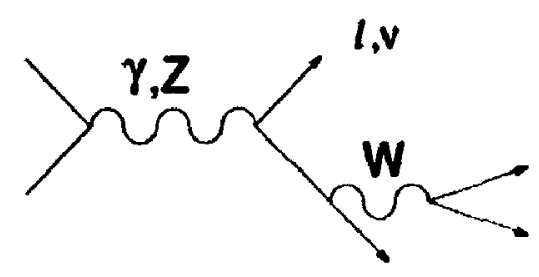

(b)

Figure 3.2: Vertex independent diagrams that contribute to all four pestible four lepesu finial state configurations

The $\mu^{+} e^{-}$final state proceeds via 18 diagrans, 4 of which contain the verticus in cinest Jont Two of these four are the W-production processes. Figure (3.1), the additional contribulticns as". from two diagrams with $a \gamma(Z)$ and a $W$ in the t-rhannel; their form is shown in Figure (3.3a) 


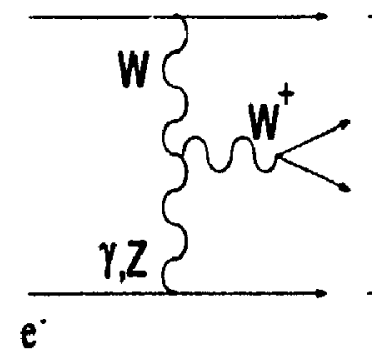

(a)

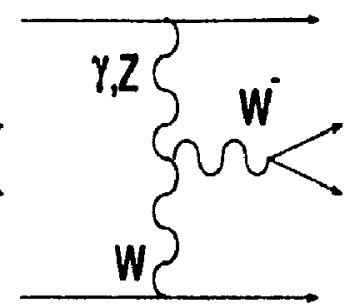

(b)

Figure 3.3: Vertex dependent diagrams that contribute to (a) $\mu^{+} e^{-}$and $e^{+} e^{-}$configurations ant (b) $e^{+} e^{-}$configuration.

For the $14 \kappa v$-independent contributing diagrams, 7 are the same as for :he $\mu^{+} \tau^{-}$state; the extra contributions are shown in Figure (3.4).

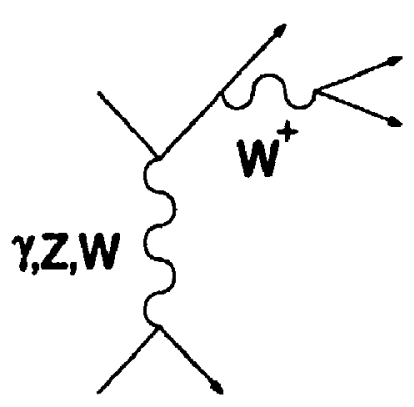

(a)

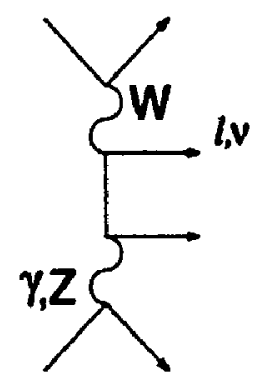

(b)

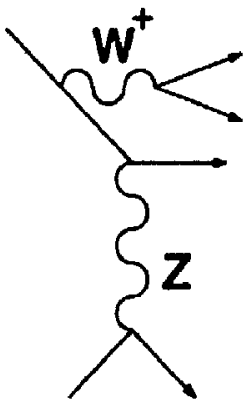

(c)

Figure 3.4: Vertex independent diagrams that contribute to the $\mu^{+} e^{-}$and $e^{+} e^{-}$final state configurations

Each diagram in Figure (3.4) represents a set of possibilities. For example, in Figure (3.4a), the $W$ boson can be emitted from either of the two top fermion lines (our labelling convention is that the incoming positron is the "top" fermion line) and the t-channel boson is then chosen accordingly. 
For the process $e^{+} e^{-} \rightarrow \mu^{+} \nu \mu^{-} \bar{\nu}, 4$ of the total 28 diagrams are sensitive to the triple boson vertices. In addition to the two s-channel $\mathrm{W}$ pair diagrams of Figure (3.1), there are two diagrams with $\mathrm{W}$ bosons in the t-channel coupled to a photon or $\mathrm{Z}$ decaying to charged leptons. 'I'hese diagrams are shown in Figure (3.5).

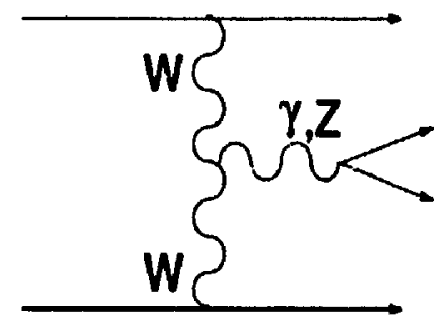

Figure 3.5: Vertex dependent diagrams that contribute to the $\mu^{+} \mu^{-}$and $e^{+} e^{-}$states.

The remaining 24 parameter independent diagrams consist of those seven from Figure (3.2), and the extras mainly being $\gamma$ or $\mathrm{Z}$ bremssirahlung from the initial or final state leptons, shown in Figure (3.6).

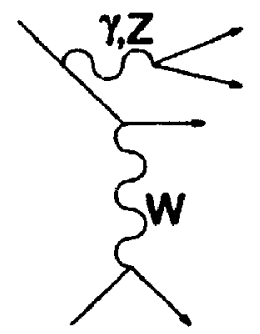

(a)

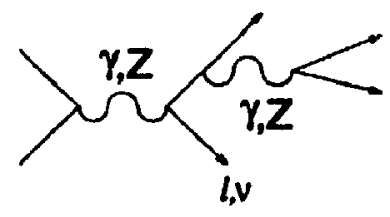

(b)

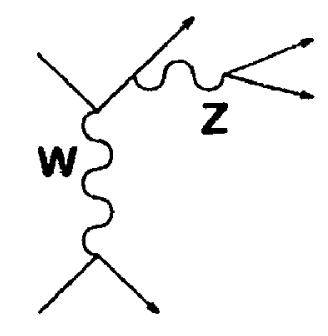

(c)

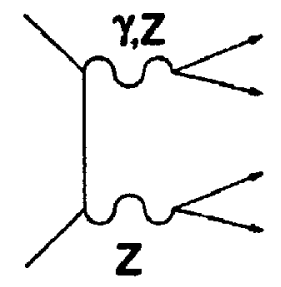

(d)

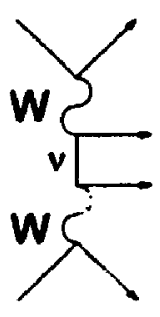

(e)

Figure 3.6: Vertex independent diagrams that contribute to $\mu^{+} \mu^{-}$and $e^{+} e^{-}$states. 
For a diagram like Figure (3.6d), where one or the other of the $\mathrm{Z}$ bosons can decay to a neutrino-antineutrino pair, all three $\nu$ flavours could appear and would be experimentally indistinguishable. These extra neutrino diagrams are added incoherently to the squared matrix element.

There are a total of 56 diagrams that contribute to the $e^{+} e^{-}$final state, 8 of which include the vertices $\gamma \mathrm{WW}$ or $\mathrm{ZWW}$. These parameter sensitive diagrams include the $\mathrm{W}$ pair production, Figure (3.1) the t-channel $\gamma(Z)$ coupled to the incoming $e^{-}$, Figure (3.3a), the 7-channel $W$ bosons, Figure (3.5), and also the two diagrams of Figure (3.3b), where the t-channel $\gamma(\mathrm{Z})$ couples to the incoming $e^{+}$. The non-sensitive contributions to this $e^{+} e^{-} \rightarrow e^{+} \nu e^{-} \bar{\nu}$ process are those previously described for the simpler final states, Figures (3.2), (3.4), and (3.6), as well as some diagrams, shown in Figure (3.7), that contribute only to the $e^{+} e^{-}$channel.

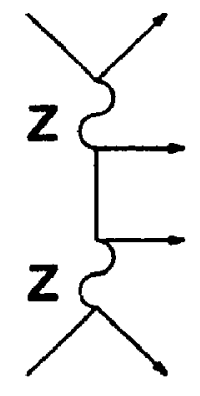

(a)

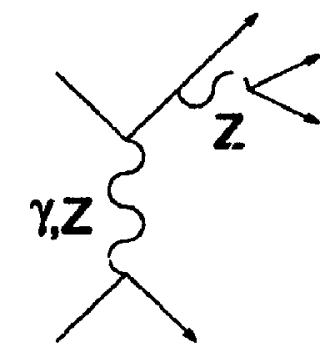

(b)

Figure 3.7: Vertex independent diagrams that contribute only to the $e^{+} e^{-}$final state configuration.

\subsection{Helicity Amplitudes}

Once the contributing diagrams for each final state are known, the contributing helicity amplitudes for each of the four processes can be determined. Our labelling convention is to denote the helicities of the incoming positron, incoming electron, outgoing charged antilepton, and outgoing charged lepton as $(\bar{\alpha} \beta \bar{p} \psi)$, respectively. Since the $\gamma(Z)$ couples to botin left and right handed fermions, and the $W$ to only the left handed variety, we see that the $W$ pair production diagram of Figure (3.1) has both $(+-+-)$ and $(-++-)$ helicity amplitudes. For the remaining 7 
vertex independent diagrams, Figure (3.2), we need tot introduce any other helicity amplitudes; thus the $\mu^{+} \tau^{-}$process has only these two contributing helicity amplitudes. Berause the $W$ pair production contributes to all tour processes, these two helicity amplitudes contribute to all four. Indeed, because $(+-+-)$ includes the cortribution from the t-channel neutrino exchange. Figure (3.2a), it is the dominant helicity amplitude in all cases. The additional diagrams for $\mu^{+} e^{-}, \mu^{+} \mu^{-}$, and $e^{+} e^{-}$provide contributions to helicity amplitudes other than these two.

The $\mu^{+} e^{-}$process includes the t-channel $\gamma(\mathrm{Z})$ diagram of Figure (3.3a) which has contributions from $(+-+-)$ and $(++++)$ amplitudes. No other amplitudes are introduced for the nondependent contributions to $\mu^{+} e^{-}$, Figure (3.4); thus there are three contributing helicity amplitudes for that process.

Figure (3.5) contributes helicities $(+-+-)$ and $(+--+)$ to the $\mu^{+} \mu^{-}$final state. In addition, the amplitude $(-+-+)$ receives contributions from some of the independent diagrams, Figure (3.6). The $\mu^{+} \mu^{-}$process therefore has four contributing amplitudes.

The $e^{+} e^{-}$process has the vertex sensitive contributions of Figure (3.3b), to the $(+-+-)$ and (- - - ) amplitudes. Because the $e^{+} e^{-}$state includes all the diagrams that contribute to the other processes, it goes via all six possible helicity amplitudes. We summarize the helicity amplitudes that contribute to the four different processes below

$$
\begin{array}{ll}
\mu^{+} \tau^{-} & (+-+-),(-++-) \\
\mu^{+} \varepsilon^{-} & (+-+-),(-++-),(++++) \\
\mu^{+} \mu^{-} & (+-+-),(-++-),(+--+),(-+-+) \\
e^{+} e^{-} & (+-+-),(-++-),(++++),(+--+)(-+-+),(----)
\end{array}
$$

The $(-+-+)$ amplitude, present for the $\mu^{+} \mu^{-}$and $e^{+} e^{-}$cases, contains no vertex sensitivity; it arises only from the vertex independent diagrams of Figures (3.6) and (3.7).

\subsection{The Calculation}

In this calculation, for each of the four different processcs, the ccitributing diagrams' amplitudes were expressed in the CALKUL, formalism [58]. In this formalism. the individual diagram amplitudes are each exn:esed as combinations of momerıta scalar products; this simple form is conducive $t_{1}$, omputatınal ease. Also, the LALKUL method retains helicity information; the 
separation of a diagrams' contribution into different helicity amplitudes is explicitly expressed and the relative contributions of these amplitudes to the total cross-section can be directly investigated. In Appendix (C) we discuss in detail the CALKUL formalism and, as an example, provide a complete calculation of an amplitude. In determining the amplitude expressions, we retain fermion masses in the propagators but neglect terms proportional to fermion masses $\boldsymbol{m}_{\boldsymbol{f}}$ in the numerators. At the high energies to be considered, this is a valid approximation. To determine the total cross-section and distributions, the matrix element squared was embedded in an adaptive Monte Carlo algorithm, VEGAS [59], for integration over the final state four-body phase space. We discuss the Monte Carlo method and the details of the VEGAS algorithm in Appendix (E). We use as input parameter values $M_{Z}=91.196 \mathrm{GeV}, \Gamma_{Z}=2.534 \mathrm{GeV}, M_{W}=$ $80.6 \mathrm{GeV}, \Gamma_{W}=2.25 \mathrm{GeV}, m_{e}=0.511 \mathrm{MeV}, m_{\mu}=0.1057 \mathrm{GeV}, m_{\tau}=1.784 \mathrm{GeV}$, and $\sin ^{2} \theta_{w}=0.23$.

The experimental signal for these processes consists of an energetic oppositely charged lepton pair plus missing transverse momentum and energy due to the undetected neutrinos. One potential background is the leptonic decay of $\tau^{\prime}$ 's produced by the $e^{+} e^{-} \rightarrow \tau^{+} \tau^{-}$process. At $\sqrt{s}=200 \mathrm{GeV}$, the four lepton processes and the $\tau$ pair production, multiplied by the branching ratios of $\tau$ into $e$ or $\mu$ of $17.8 \%$ each [60], yield approximately the same rate. At higher energies however, the $\tau$ pair production cross-section falls like $1 / \mathrm{s}$ while the cross-sections for our four lepton processes remain sizeable. Additionally, with four final state neutrinos, this process should be distinguishable by its greater amounts of missing energy and transverse momenta, compared to our processes of interest.

Also, the leptonic decay of one or more $\tau^{\prime}$ in the final states $\mu^{+} \tau^{-}$and $\tau^{+} \tau^{-}$could simulate the occurence of one of the other processes, such as $\mu^{+} e^{-}$or $\mu^{+} \mu^{-}$. However, the lepton branching ratio of $17.8 \%$ for the $\tau$, and the greater missing energy and transverse momenta from the extra neutrinos, prevent this background from being problematic.

The "like-lepton" final states $\mu^{+} \mu^{-}$and $e^{+} e^{-}$have a possible background from two photon processes where the electron and positron are undetected along the beam. With no final state neutrinos, there should be no characteristic missing transverse momentum. Thus, we can minimize these two photon events as a background source by requiring a minimum amount of missing transverse momentum. This is, of course, equivalent to requiring a minimum for the visible transverse momentum. Therefore, for the final states $\mu^{+} \mu^{-}$and $e^{+} e^{-}$, a cut requiring total visible momentum $p_{T}>10 \mathrm{GeV}$ was implemented. Also, we required for these "like-lepton" processes that each charged lepton have a minimum energy, $E_{l}>10 \mathrm{GeV}$, and that the invariani 
mass of the charged lepton pair exceed $25 \mathrm{GeV}$. This cut serves to regulate the low invariant mass leptons arising from the photon pole in these processes.

In addition to these process specific cuts, we also made simple cuts to account for limitations on detector acceptance, such as beam pipe restrictions. To more realistically describe the experimentally accessible phase space, we cut on the angle of each of the charged leptons relative to the beam line such that $-0.95<\cos \theta_{l}<0.95$. This is equivalent to requiring the charged leptons to be emitted with an angle with respect to the beam greater than approximately $18^{\circ}$. Although motivated by experimental considerations, this cut also serves to regulaie the t-channel photon pole from the diagrams of Figures (3.3a) and (3.3b). Including the fermion masses, the photon propagator from these diagrams can be written as

$$
\frac{1}{(k-p)^{2}}=\frac{1}{m_{k}^{2}+m_{p}^{2}-2 E_{k} E_{p}\left(1-\cos \theta_{k p}\right)}
$$

where $k$ is the momentum of the incoming electron(positron) and $p$ that of the outgoing lepton(antilepton). The cut $-0.95<\cos \theta_{l}<0.95$, by preventing the propagator from "blowing-up" when $\cos \theta_{k p}=1.0$, allows us to neglect the fermion masses throughout our calculation.

In addition to determining total cross-sections, we generated various distributions. These include variables such as the angles of the charged leptons relative to the beam $\theta_{ \pm}$, the opening angle between the charged leptons $\theta_{+-}$, the energies, individual and total, of the visible leptons, $E_{ \pm}$and $E_{T}$, the total visible transverse momenta $p_{T}$, and the invariant mass of the charged lepton pair $M_{l_{+} l_{-}}$. As well as these distributions, we generated others which, although not experimenially observable, provided important checks on the consistency of our results. For instance, because of the neutrinos, the angular and invariant mass distributions of the reconstructed W's are not experimentally accessible. Nevertheless, by cutting on these invariant masses for the $\mu^{+} \tau^{-}$channel, we can effectively generate results for $e^{+} e^{-} \rightarrow W^{+} W^{-}$. The $\mu^{+} \tau^{-}$process includes the three $W$ pair production diagrams; by requiring the invariant masses of the two lepton-neutrino pairs to approximate the $\mathrm{W}$ boson mass, $M_{W}-5 \mathrm{GeV}<M_{\mu r}<M_{W}+5$ (ieV. the contributions extra to $W$-pair production are largely excluded. Our $\mu^{+} \tau^{-}$results, with these. restrictions, do reproduce thoee of $W$-pair production, $[20,30,61]$.

The requirements imposed by charge conjugation symmetry provided a further check on the self consistency of our results. The channels with $\mu^{+} \tau^{-}$(neglecting the mass difference), $\mu^{+} \mu^{-}$, and $e^{+} e^{-}$in the final state are all individually charge conjugate symmetric. The $\mu^{+} e^{-}$ channel differentiates between the $\mu$ and the $e$, so charge symmetry requires both $\mu^{+} e^{-}$and 
$\mu^{-} e^{+}$channels. To verify this symmetry, various redundant distributions and charge asymmetries were generated for the positively and negatively charged leptons. These ideas are discussed in Appendix (E).

In Chapter 4, we investigate the sensitivity of the total cross-section and the vario's distributions of the four processes to the CP respecting couplings $\kappa_{V}$. Motivated by the theoretical predictions for the relative smallness of the deviation $\Delta \lambda$, we reglect it, and concentrate on the couplings $\kappa_{\gamma}$ and $\kappa_{z}$. We leave for Chapter $\vec{i}$ the possibility of a CP violating contribution in the triple boson vertex. We perform our investigation of the $\kappa_{V}$ dependence at two center-of-mass energies, $\sqrt{s}=500 \mathrm{GeV}$ and $\sqrt{8}=1 \mathrm{TeV}$. The theoretical predictions for the expected deviations are typically at the percent level [2i, 22], thus we vary $\kappa_{\gamma}$ and $\kappa_{z}$, both singly and together, within $10 \%$ of 1 . We repeat this analysis for each of the four channels of four lepton production at both of the two energies. 


\section{Chapter 4}

\section{Deriving Limits for $\kappa_{V}$}

In this Chapter we examine the sensitivity of the total cross-sections and the observable distributions to variations in the triple boson couplings $\kappa_{\gamma}$ and $\kappa_{z}$. We make our study at the two center-of-mass energies, $500 \mathrm{GeV}$ and $1 \mathrm{TeV}$. In Figure (4.1) we show the cross-sections as functions of $\sqrt{s}$ for the $\mu^{+} \tau^{-}, \mu^{+} e^{-}, \mu^{+} \mu^{-}$, and $e^{+} e^{-}$channels respectively.
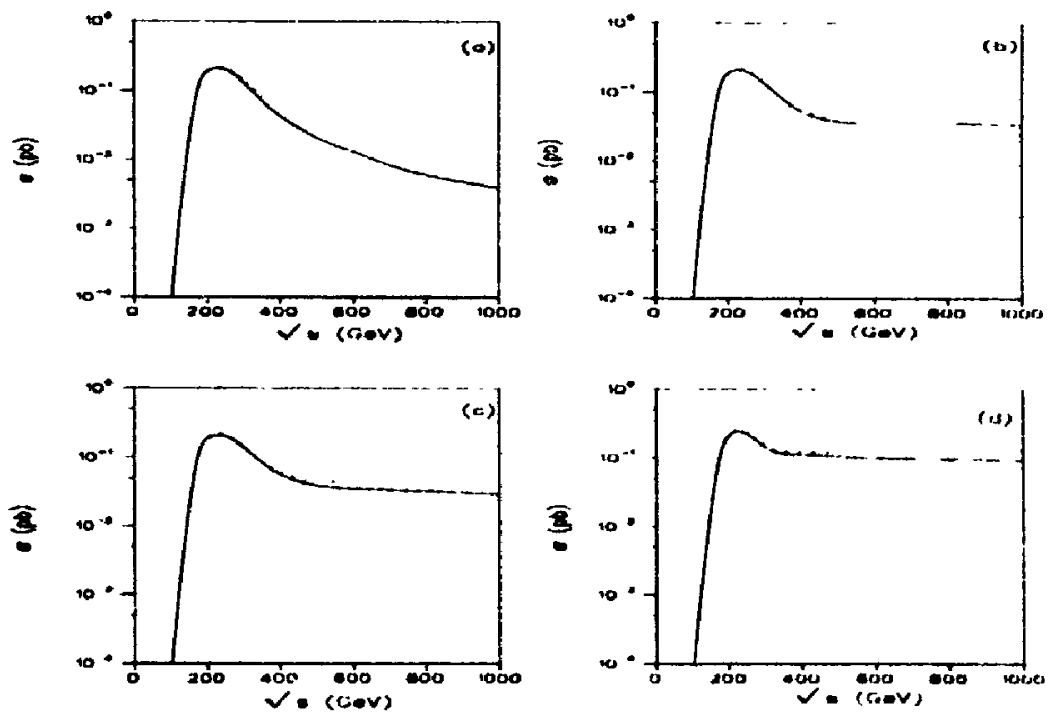

Figure 4.1: Cross-section as function of center of mass energy for (a) $\mu^{+} \tau^{-},(b) \mu^{+} \mu^{-},(c) \mu^{+} \mu^{-}$, and (d) $e^{+} e^{-}$channels. The solid line corresponds to the Standard Model, the dotted line to $\kappa_{\gamma}=\kappa_{z}=0.9$ 
The standard model cross-section exhibits the unitarity respecting high energy behaviour rharacteristic of gauge theories. The precise cancellations that produce this decrease in crosssertion with $\sqrt{s}$ are however, destroyed when the couplings take values other than those prescribed by the gauge structure. The result is a cross-section that rises with center of mass energy, as is visible from the curves for $\kappa_{\gamma}=\kappa_{Z}=0.9$. When one or the other of $\kappa_{\gamma}$ and $\kappa_{Z}$ is kept at its SM value, and the second is allowed to vary independently, the cross-section exhibits the same behaviour, although not as markedly. The advantage in going to higher center of mass energies is apparent from the curves; the discrepancy between the SM cross-section, $\sigma_{S M}$, and that obtained with non-standard couplings, $\sigma_{N S M}$, increases with $\sqrt{8}$. Thus, although the smaller $\sigma_{S M}$ at higher energies means fewer expected events, and consequently poorer statistics; the increased sensitivity to the $\alpha V$ couplings makes studies at higher energies worthwhile.

Although the $\mu^{+} \boldsymbol{r}^{-}$process has the smallest cross-section of the four channels at high energies, it shows the greatest sensitivity to $\mathrm{KV}$. With 2 of its 9 diagrams containing the triple boson vertex, compared to, for instance, 8 of 56 for the $e^{+} e^{-}$channel, this is as might be expected. The $e^{+} e^{-}$ channel, with the greatest cross-section at higher energies and hence best statistics, exhibits the least sensitivity. The smaller cross-section for $\mu^{+} \tau^{-}$at high energies is also indicative of greater simplicity; the sensitivity to $\kappa_{V}$ arises from the two s-channel $\gamma$ or $Z$ diagrams. The $\mu^{+} \tau^{-}$channel therefore offers the clearest window into $\kappa V$ effects. The $\mu^{+} e^{-}$process, with crose-sections and sensitivities between the extremes of $\mu^{+} \boldsymbol{r}^{-}$and $e^{+} e^{-}$, is the first to include the t-channel $\kappa$-dependent diagrams of Figure (3.3a). The $\mu^{+} \mu^{-}$process offers the possibility of isolating the ZWW vertex by cutting on the invariant mass of the $\mu^{+} \mu^{-}$pair. Therefore, it is useful to consider all four processes; they each access different information on the triple boson vertices.

In Section 4.1 we determine limits from total cross-section measurements, with a discussion of the effect of including systematic errors and the scaling behaviour of the sensitivity to nonstandard couplings. Various differential cross-sections are examined in Section 4.2, motivating the ides of improving the detection limits by excluding the non $\kappa_{V}$-dependent regions of phase space, examined in Section 4.3. In Section 4.4 we explore the possibility of disentangling the effects of the WWy and WWZ vertices. 


\subsection{Limits from total cross-section measurements}

For each pairing of non-standard $\left(\kappa_{\gamma}, \kappa_{z}\right)$ values, we are concerned with the ratio of the nonstandard total cross-section to that of the Standard Model. With both $\kappa_{r}$ and $\kappa_{z}$ ranging from 0.9 to 1.1 , we determine a grid of these non-standard cross-section ratios for each of our four processes, and at the center of mass energies of $500 \mathrm{GeV}$ and $1 \mathrm{TeV}$. To determine the complete grid, it was sufficient to examine the following subset; the three cases where

$$
\begin{aligned}
& \text { 1) } \kappa_{\gamma}=\kappa_{z} \quad \text { ranging from } 0.9 \rightarrow 1.1 \\
& \text { 2) } \kappa_{\gamma}=1.0, \kappa_{z} \text { ranging from } 0.9 \rightarrow 1.1 \\
& \text { 3) } \kappa_{z}=1.0, \kappa_{\gamma} \text { ranging from } 0.9 \rightarrow 1.1
\end{aligned}
$$

For each of these three cases, the amplitude for a given process can be written as the sum of two pieces; one proportional to $\kappa_{V}$, and a $\kappa_{V}$ independent piece.

$$
M=a \kappa_{v}+b
$$

The cross-section, proportional to the the square of the amplitude $M$, will then be a parabolic function of $\kappa_{V}$. In Figures (4.2) and (4.3) we show the ratio $R=\sigma / \sigma_{S M}$ for each of the four processes at $\sqrt{s}$ of $500 \mathrm{GeV}$ and $1 \mathrm{TeV}$, respectively, for the three cases listed above. In earh diagram, the solid line corresonds to $\kappa_{\gamma}=\kappa_{z}$, the dashed line to $\kappa_{z}=1.0$, and the dotted line to $\kappa_{\gamma}=1$.0. For reference, the approximate standard model cross-section is given for each of the processes. 

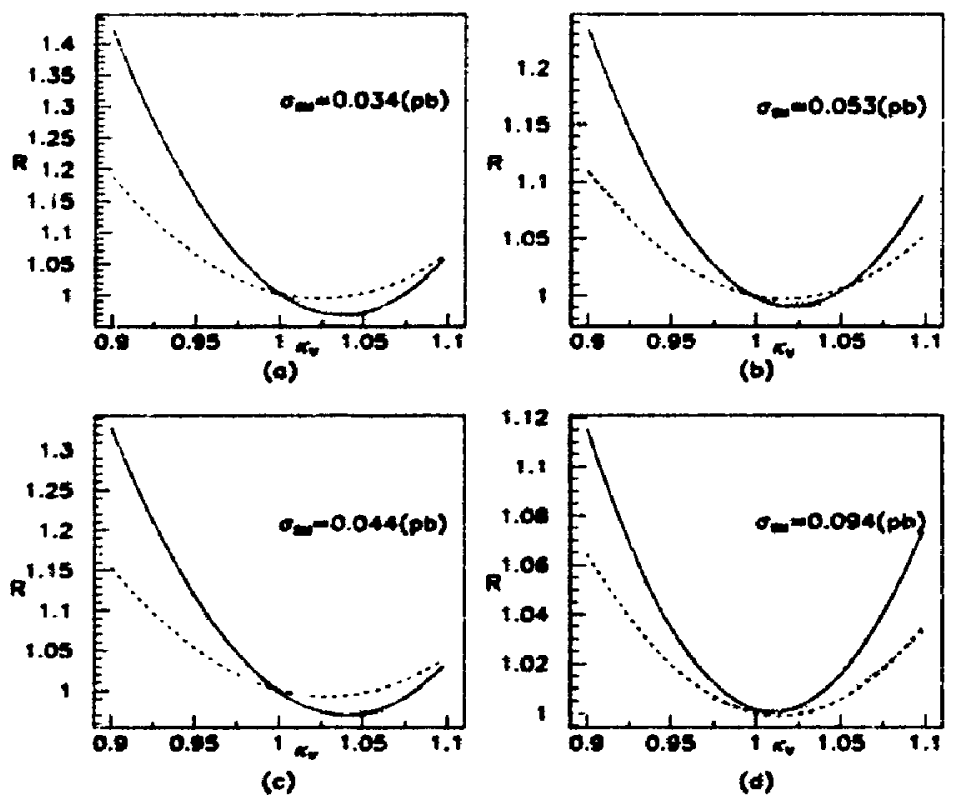

Figure 4.2: Ratio of non-standard to standard crose-section as function of $\kappa_{\gamma}$ and $\kappa_{z}$ at $500 \mathrm{GeV}$ for (a) $\mu^{+} \tau^{-}$, (b) $\mu^{+} e^{-}$, (c) $\mu^{+} \mu^{-}$, and (d) $e^{+} e^{-}$channels. The solid line is $\kappa_{\gamma}=\kappa_{z}$, the dashed line is $\kappa_{z}=1.0$, and the dotted line is $\kappa_{\gamma}=1.0$. The standard model crose-section is given for reference. 


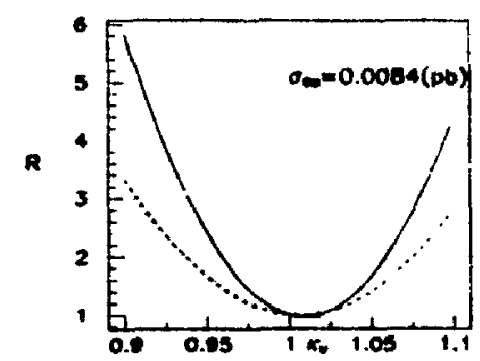

(a)

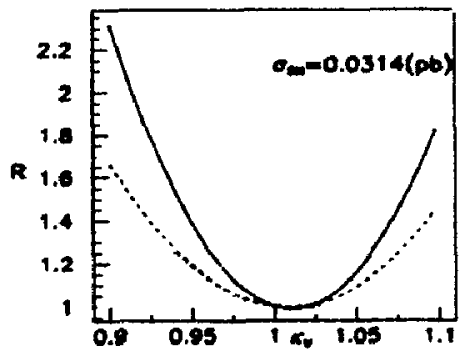

(c)

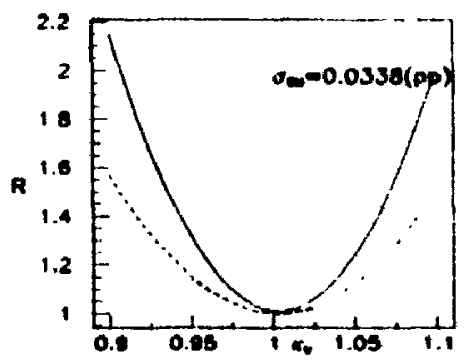

(b)

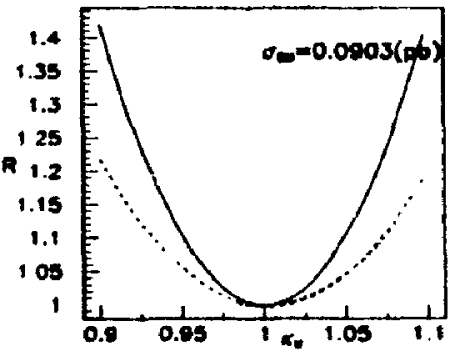

(d)

Figure 4.3: Ratio of non-standard to standard cross-section as function of $\kappa_{\gamma}$ and $\kappa_{z}$ at 1000 $\mathrm{GeV}$ for (a) $\mu^{+} \tau^{-},(\mathrm{b}) \mu^{+} e^{-},(\mathrm{c}) \mu^{+} \mu^{-}$, and (d) $e^{+} e^{-}$channels. The solid line is $\kappa_{\gamma}=\kappa_{Z}$, the dashed line is $\kappa_{z}=1.0$, and the dotted line is $\kappa_{\gamma}=1.0$. The standard model cross-section is given for reference. 
We from these curves that the case of $\kappa_{\gamma}=\kappa_{z}$ displays the greatest sensitivity to deviations of $\kappa_{V}$ from unity. This is not surprising; with one or the other of $\kappa_{\gamma}$ and $\kappa_{z}$ kept at its SM value, we expect less of a deviation from the SM cross-section. Another general feature is that, at the lower energy of $500 \mathrm{GeV}$, each process shows greater sensitivity to variations $\Delta \kappa_{V}=\kappa_{V}-1<0$ than to $\Delta \kappa_{V}>0$. For $\kappa_{\gamma}$ or $\kappa_{Z}$ varied individually we can parametrize the deviation of the non-standard cross-section from the SM value by

$$
\begin{aligned}
& \Delta \sigma=\sigma-\sigma_{S M} \\
& \Delta \sigma=a+b x+c i^{2}-(a+b+c) \\
& \Delta \sigma=a+b(\Delta x+1)+c(\Delta \kappa+1)^{2}-(a+b+c) \\
& \Delta \sigma=(b+2 c) \Delta \kappa+c(\Delta \kappa)^{2}
\end{aligned}
$$

The observed asymmetries in $\Delta \sigma$ at $\sqrt{s}=500 \mathrm{GeV}$ can only arise from the term linear in $\Delta \kappa v$, and we must have $b+2 c<0$. At the higher center of mass energy of $1 \mathrm{TeV}$, the sensitivity is. much more symmetrical about 1 . The symmetry of the curves of Figure (4.3) imply that, at 1 $\mathrm{TeV}, b+2 c \sim 0$, and the term quadratic in $\Delta \boldsymbol{k}_{\mathrm{V}}$ dominates.

The cross-section, expressed as a multivariable function of $\kappa_{\gamma}$ and $\kappa_{z}$, without the previous restrictions, takes the form

$$
\sigma \sim|M|^{2}=a+b \kappa_{\gamma}+c \kappa_{Z}+d \kappa_{\gamma} \kappa_{Z}+e \kappa_{\gamma}^{2}+f \kappa_{Z}^{2}
$$

The coefficients $a, b, c, d, e$, and $f$ are determined by fitting parabolas to the three special cases, $\left(\kappa_{\gamma}=\kappa_{z}, \kappa_{\gamma}=1\right.$, and $\left.\kappa_{z}=1\right)$, and then solving the system of equations. For instance, the case of $\kappa_{\gamma}=\kappa_{Z}=\kappa_{V}$ corresponds to the diagonal of the full 3-dimensional surface where

$$
\sigma_{\gamma=Z}=a+(b+c) \kappa_{V}+(d+e+f) \kappa_{V}^{2}
$$

By equating the parameters of the curve fit to this parabola with the corresponding coefficients in the above expreseion, we obtain relations between $a, b, c, d, e$, and $f$. Similarly for the other two cases:

$$
\begin{aligned}
& \sigma_{\gamma=1}=(a+b+e)+(c+d) \kappa_{Z}+f \kappa_{Z}^{2} \\
& \sigma_{z=1}=(a+c+f)+(b+d) \kappa_{\gamma}+e \kappa_{\gamma}^{2}
\end{aligned}
$$


We obtain a set of linear equations, the solution of which gives values for the coefficients $a, b, c, d, e$, and $f$. Thesr values, substituted into the expression for the cross-sertion as a function of $\kappa_{\gamma}$ and $\kappa_{z}$, see Eq.(4.1), determine the rross-spetion as a function of $\kappa_{2}-\kappa_{2}$. Wr :vere consequently able to generate cross-section values over the full $\kappa_{2}-\kappa_{z}$ gricl, without the use of prohibitive amounts of computing time. As a check on our technique, we compared these. generated cross-sections with those obtained by direct Monte Carlo evaluation, the diserepallicins were generally of the order of $0.1 \%$. For instance, setting $\alpha_{\gamma}=\kappa_{z}=0.9$ for the $\mu^{i} r^{-}$.lianurl at $\sqrt{s}=500 \mathrm{GeV}$, the total cross-sections from direct Monte (ario and the fitting proctess are $0.0486(\mathrm{pb})$ and $0.0487(\mathrm{pb})$, respectively; a difference of $0.2 \%$. Such small discrepanciesi will mot. effect our results for the detection bounds on $\kappa_{\gamma}, \kappa_{z}$ to any significant level; our approximation therefore seems valid. The resulting surface plots for the cross-sections of the $\mu^{+} \tau^{-}, \mu^{+} \mu^{-}$, $\mu^{+} \mu^{-}$, and $e^{+} e^{-}$channels are shown in Figures (4.4) and (4.5), for $\sqrt{s}$ of 5 (J) (ieV and $I T \cdot V$ respectively. 

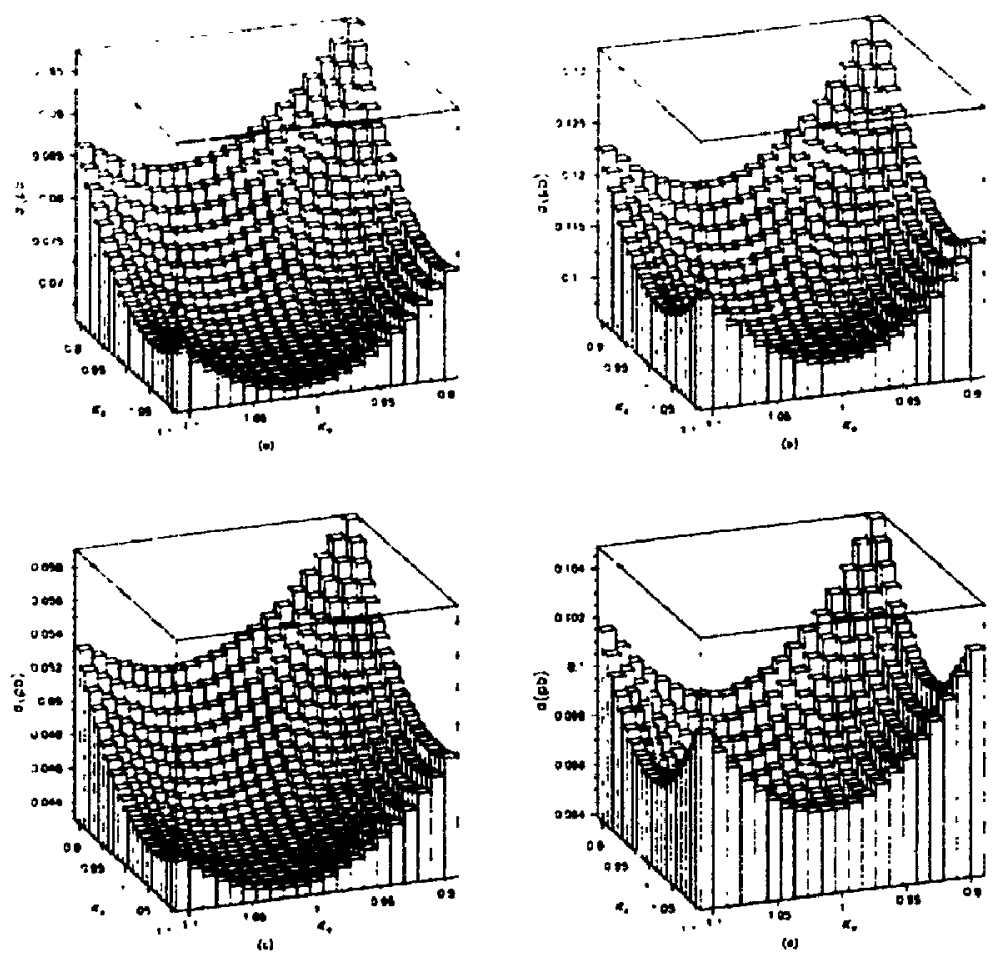

Figure 4.4: Lego plots in $\kappa_{\gamma}$ and $\kappa_{Z}$ for (a) $\mu^{+} \tau^{-}$, (b) $\mu^{+} e^{-}$, (c) $\mu^{+} \mu^{-}$, and (d) $e^{+} e^{-}$channels at $\sqrt{s}=500 \mathrm{GeV}$ 

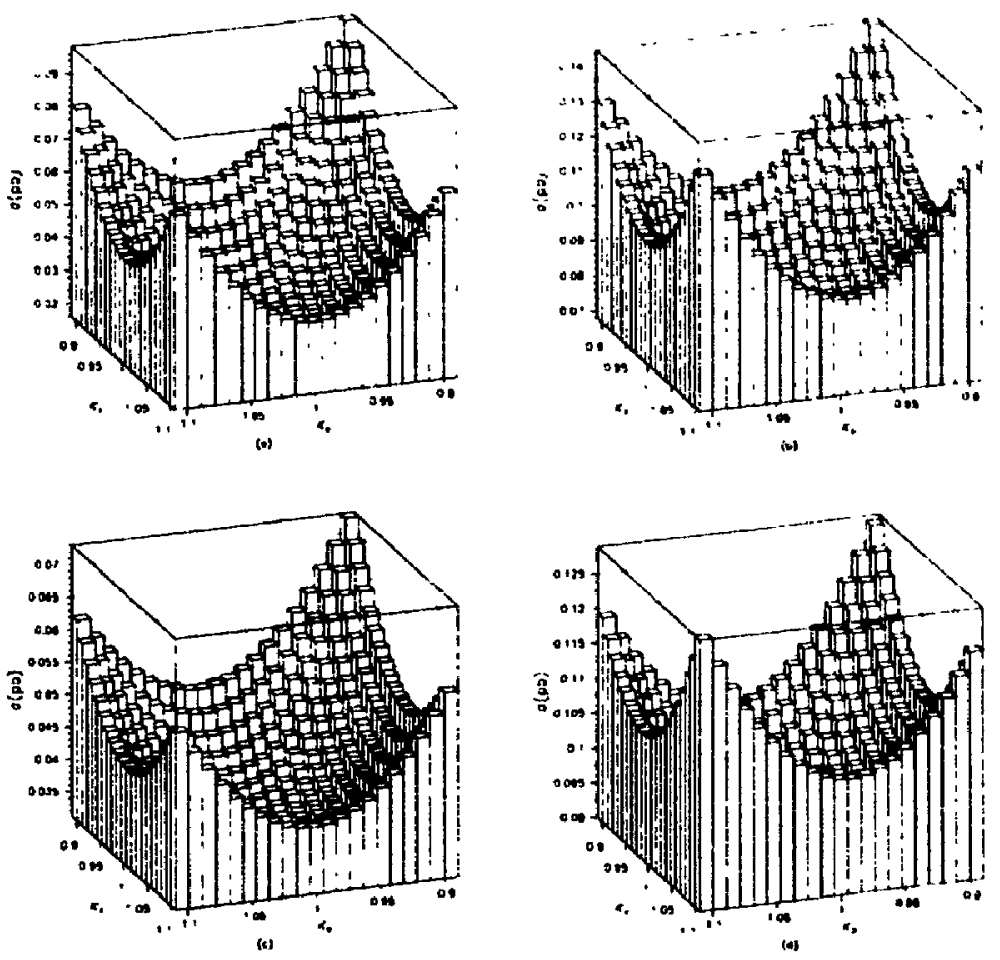

Figure 4.5: Lego plots in $\kappa_{\gamma}$ and $\kappa_{Z}$ for (a) $\mu^{+} \tau^{-}$, (b) $\mu^{+} e^{-}$, (c) $\mu^{+} \mu^{-}$, and (d) $e^{+} e^{-}$shannels at $\sqrt{s}=1000 \mathrm{GeV}$ 
We again observe the sensitivity asymmetry for the lower energy; it is most marked for the $\mu^{+} \tau^{-}$plot. There, the difference in sensitivities between the quadrants $(0.9 \rightarrow 1.0,0.9 \rightarrow 1.0)$ and $(1.0 \rightarrow 1.1,1.0 \rightarrow 11)$ is extreme. In the former quadrant, the cross-section is significantly enhanced relative to $\sigma_{S M}$; in the latter, it shows little sensitivity at all. In part of this quadrant the cross-section is slightly smaller than that of the SM. This fact could prove useful in identifying the source of a new physics signal; a cross-section below the standard model value would indicate values for the $\kappa_{V}$ 's in the fourth quadrant. At the higher energy of $1 \mathrm{TeV}$ any non-standard pairing results in an enhanced cross-section, relative to that of the SM. Thus, $\sigma_{S M}$ lies at the minimum of the surface plot, and no such discrimination is possible.

Also apparent from the surface plots is the approximate symmctry about the diagonal $\kappa_{\gamma}=\kappa_{Z}$. Because, for every diagram involving the $\gamma \mathrm{WW}$ vertex, there is a partner with the $\mathrm{ZWW}$ vertex, the only variables that differentiate between $\kappa_{\gamma}$ and $\kappa_{Z}$ are the overall relative couplings and mass differences. This diagonal symmetry is improved at $1 \mathrm{TeV}$, where the photon- $\mathrm{Z}$ mass difference becomes less significant.

We derive limits on the deviations $\Delta \kappa_{\gamma}$ and $\Delta \kappa_{Z}$ by determining the statistical $2 \sigma$ range about the standard model cross-section. This value, multiplied by the integrated luminosity (we assume $L=\int \mathcal{C} d t=50 \mathrm{fb}^{-1}$ ), determines the "expected" number of events, $N_{S M}$. By assuming a statistical error of $\sqrt{N_{S M}}$ about this number, we determine the $2 \sigma$ range for the "expected" number of events, $N_{S M} \pm 2 \sqrt{N_{S M}}$. From this, we work backwards to determine the $2 \sigma$ range about the standard model cross-section, $\sigma_{S M} \pm 2 \sigma_{\sigma_{S M}}$. We interpret this as the range of crosssections that are "statistically indistinguishable" from the standard model value. Thus, for a non-standard pairing of $\kappa_{\gamma}$ and $\kappa_{z}$ to give a statistically significant signal, it must result in a cross-section outside the given range. This required minimum cross-secticn deviation determines limits on the deviations $\Delta \kappa_{y}$ and $\Delta \kappa_{Z}$. We determine these limits by plotting the $\pm 2 \sigma_{\sigma_{S M}}$ values against the 3-D surface plots of Figures (4.4) and (4.5). These cross-section extremes determine $2 \sigma$ contcurs, as shown in Figures (4.6) and (4.7), for the two center of mass energies respectively. We show also in Figures (4.6) and (4.7) the $1 \sigma$ contours, the plots with less than four contours are those in which the $-2 \sigma$ or $-1 \sigma$ cross-sections were below the minimum of the 3-D surface, and so were irrelevant. In deriving these limits, we multiplied the standard model cross-section for the $\mu^{+} \tau^{-}$and $\mu^{+} e^{-}$processes by a factor of two to account for the charge conjugate processes. 

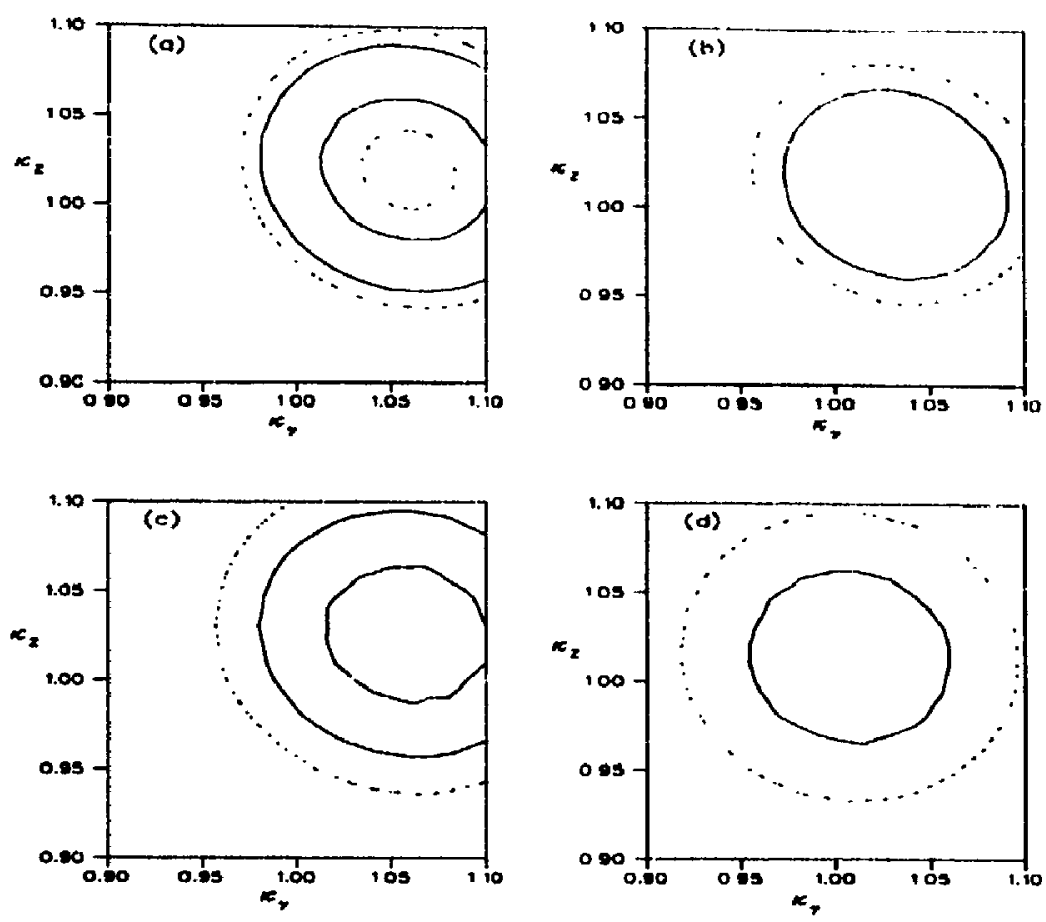

Figure 4.6: $1 \sigma$ (solid lines) and $2 \sigma$ contours (dot-dashed lines) in $\kappa_{\gamma}$ and $\kappa_{Z}$ for (a) $\mu \tau$, (b) $\mu c^{\prime}$, (c) $\mu^{+} \mu^{-}$, and (d) $e^{+} e^{-}$channels at $\sqrt{s}=500 \mathrm{GeV}$. 

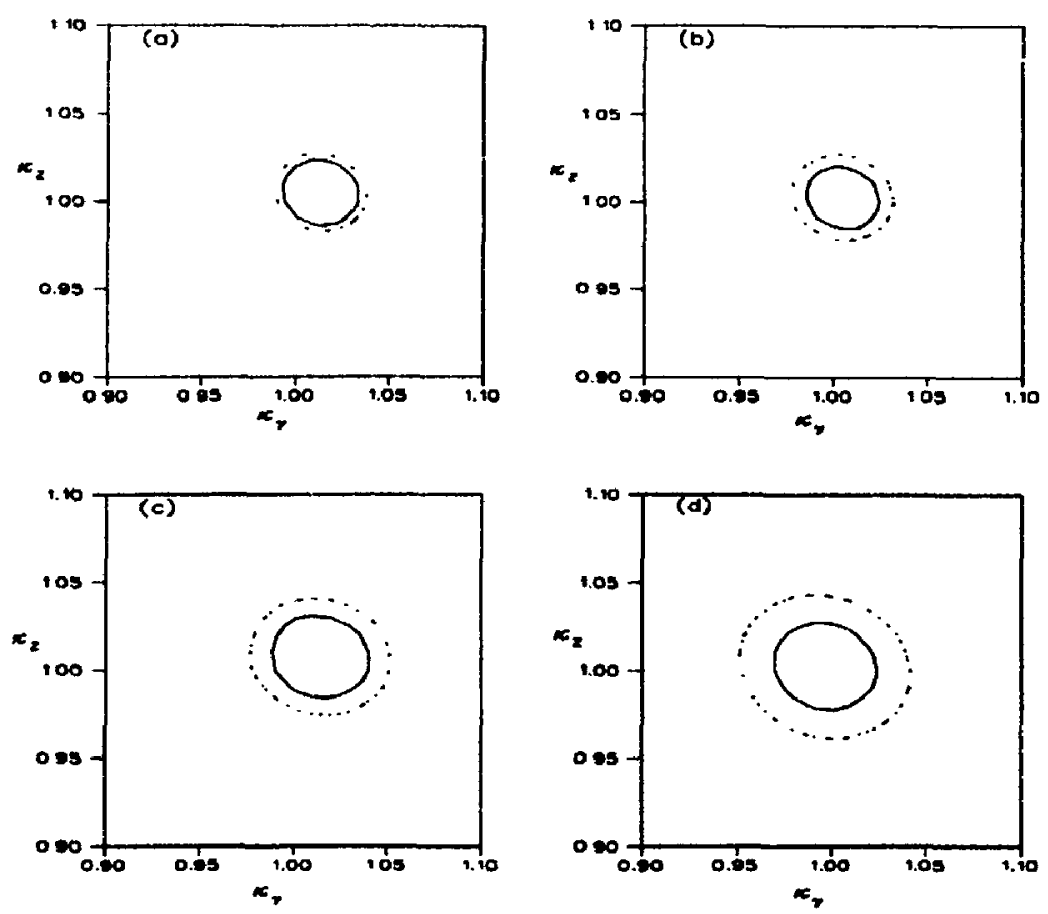

Figure 4.7: $1 \sigma$ (solid lines) and $2 \sigma$ contours (dot-dashed lines) in $\kappa_{\gamma}$ and $\kappa_{Z}$ for (a) $\mu \tau$, (b) $\mu e$, (c) $\mu^{+} \mu^{-}$, and (d) $e^{+} e^{-}$channels at $\sqrt{s}=1 \mathrm{TeV}$. 
We list in Table 4.1 the limits deternined from the $+2 \sigma$ contour plots of Figures $(4.6)$ and (4.7).

Table 4.1: $95 \%$ C.L. bounds on non-standard couplings $\mathrm{Ni}_{\mathrm{i}}$ about Standard Model $\mathrm{ki}_{\mathrm{V}}=1.0$

\begin{tabular}{|c|c|c|c|}
\hline \multirow[t]{4}{*}{$\mu^{+} \tau^{-}$} & \multirow[t]{2}{*}{$500 \mathrm{GeV}$} & $n_{\gamma}$ & $0.975 \leftrightarrow 1.15$ \\
\hline & & $\kappa_{z}$ & $0.94 \leftrightarrow 1.1$ \\
\hline & \multirow[t]{2}{*}{$1000 \mathrm{GeV}$} & $n_{i}$ & $0.99 \leftrightarrow 1.035$ \\
\hline & & $\kappa_{z}$ & $0.98 \leftrightarrow 1.025$ \\
\hline \multirow[t]{4}{*}{$\mu^{+} e^{-}$} & \multirow[t]{2}{*}{$500 \mathrm{GeV}$} & $\kappa_{\gamma}$ & $0.955 \leftrightarrow 1.1$ \\
\hline & & $\kappa_{Z}$ & $0.955 \leftrightarrow 1.08$ \\
\hline & \multirow[t]{2}{*}{$1000 \mathrm{GeV}$} & $k_{\gamma}$ & $0.975 \leftrightarrow 1.03$ \\
\hline & & $\kappa_{Z}$ & $0.975 \leftrightarrow 1.03$ \\
\hline \multirow[t]{4}{*}{$\mu^{+} \mu^{-}$} & \multirow[t]{2}{*}{$500 \mathrm{GeV}$} & $\kappa_{\gamma}$ & $0.965 \leftrightarrow 1.15$ \\
\hline & & $\kappa_{Z}$ & $0.94 \leftrightarrow 1.11$ \\
\hline & \multirow[t]{2}{*}{$1000 \mathrm{GeV}$} & $\kappa_{\gamma}$ & $0.98 \leftrightarrow 1.05$ \\
\hline & & $\kappa_{Z}$ & $0.975 \leftrightarrow 1.035$ \\
\hline \multirow[t]{4}{*}{$e^{+} e^{-}$} & \multirow[t]{2}{*}{$500 \mathrm{GeV}$} & $\kappa_{\gamma}$ & $0.90 \leftrightarrow 1.08$ \\
\hline & & $\pi z$ & $0.93 \leftrightarrow 1.095$ \\
\hline & \multirow[t]{2}{*}{$1000 \mathrm{GeV}$} & $\kappa_{\gamma}$ & $0.95 \leftrightarrow 1.04$ \\
\hline & & $\kappa_{Z}$ & $0.97 \leftrightarrow 1.055$ \\
\hline
\end{tabular}

From Table 4.1 we extract the "best" limits, expressing them as percent deviations. At $\sqrt{s}=500 \mathrm{GeV}$, we found

$$
\begin{aligned}
& -2.5 \%(\mu \tau)<\Delta \kappa_{\gamma}<+8.0 \%(e e) \\
& -4.5 \%(\mu e)<\Delta \kappa_{Z}<+8.0 \%(\mu e)
\end{aligned}
$$

and at $\sqrt{s}=1 \mathrm{TeV}$,

$$
\begin{array}{r}
-1.0 \%(\mu \tau)<\Delta \kappa_{\gamma}<+3.5 \%(\mu \tau, \mu e) \\
-1.5 \%(\mu \tau)<\Delta \kappa_{z}<+2.5 \%(\mu \tau)
\end{array}
$$


The channels given in parentheses with each limit indicate which of the processes supply the tightest constraint. These bounds assume that non-standard physics in the triple boson vertices will manifest itself as an enhancement of the standard model cross-section; we account only for the $+2 \sigma$ contour. This is justified at $1 \mathrm{TeV}$; there we saw that the $\sigma_{S M}$ lay at the minimum of the cross-section surface plot, thus any non-standard cross-section was greater than $\sigma_{S M}$. However, we saw that at the lower energy of $\sqrt{\delta}=500 \mathrm{GeV}$, there were possible combinations of $\kappa_{\gamma}$ and $\kappa_{Z}$ that resulted in a smaller than standard cross-section. The $\sigma_{S M}$ is not the smallest therefore, and the $\sigma_{S M}-2 \sigma_{\sigma S M}$ contour is not automatically irrelevant. We see this effect in the contours of Figure (4.6a), corresponding to the $\mu^{+} \tau^{-}$channel. The $\pm 2 \sigma$ contours define an annulus, the points within statistically indistinguishable from the standard model cross-section, the points outside represent those pairings of $\kappa_{\gamma}$ and $\kappa_{Z}$ which give a statistically significant deviation. If we were to consider only the $+2 \sigma$ contour in determining our bounds, we would neglect the potentially important region inside the annulus. Accounting for this region, we would give the $\pm 2 \sigma$ limits on $\Delta \kappa v$ as

$$
\begin{array}{r}
0.975<\kappa_{\gamma}<1.03, \quad 1.08<\kappa_{\gamma}<1.15 \\
0.94<\kappa_{Z}<0.99, \quad 1.05<\kappa_{Z}<1.1
\end{array}
$$

where the limits obtained by accounting only for the $+2 \sigma$ contour would be

$$
\begin{array}{r}
0.975<\kappa_{\gamma}<1.15 \\
0.94<\kappa_{Z}<1.1
\end{array}
$$

Apparent from the contours and the derived limits is the advantage in going to higher energies. especially to probe values of $\kappa_{V}$ larger than 1 . The loose bounds on positive $\Delta \kappa_{V}$ at $\sqrt{s}=500$ $\mathrm{GeV}$ are a consequence of the asymmetrical shape of the 3-D cross-section surfaces at this energy. The more symmetrical situation at $1 \mathrm{TeV}$, with the $\sigma_{S M}$ at the minimum of the cross-section surface, requires less of a deviation in $\kappa_{V}$ to give the $+2 \sigma$ cross-section, and consequently tighter constraints on $+\Delta x v$ are obtained.

We could potentially improve our bounds by combining all the four lepton processes. With a total of nine channels, $\mu^{+} \tau^{-}\left(\mu^{-} \tau^{+}\right), \mu^{+} e^{-}\left(\mu^{-} e^{+}\right), \tau^{+} e^{-}\left(\tau^{-} e^{+}\right), \mu^{+} \mu^{-},\left(\tau^{+} \tau^{-}\right)$, and $e^{+} e^{-}$, we expect a large number of events and improved statistics. For instance, combining the standard model cross-sections to account for the equivalent (in the massless limit the $\boldsymbol{\tau}^{+} e^{-}$channel is 
equivalent to the $\mu^{+} e^{-}$channel) and charge conjugate channels, we get for the total four lepton production cross-section

$$
\sigma=2 \sigma_{\mu \tau}+4 \sigma_{\mu \mathrm{e}}+2 \sigma_{\mu \mu}+\sigma_{\mathrm{ee}}
$$

which, at $\sqrt{s}=500 \mathrm{GeV}$, gives a total cross-section of $\sigma=0.4794 \mathrm{pb}$. Multiplying this hy the integrated luminosity of $50 \mathrm{fb}^{-1}$, we expect a large number of events, $N=23,970$. This corresponds to a $+2 \sigma$ critical sensitivity of approximately $1 \%$. This critical sensitivity is significantly lower than those of the individual processes, eg. $3.5 \%$ for $\mu^{+} \tau^{-}$at $500 \mathrm{GeV}$. Of course, by combining the processes in this way, each of which with different "physical" sensitivities, the combined physical sensitivity can be less than that of the most sensitive, the $\mu^{+} \tau^{-}$channel. Also, we lose some of the discriminatory ability provided by analyzing the processses individualiy, eg. the below standard $\sigma$ effect seen in $\mu^{+} \tau^{-}$would be swamped by the higher numbers from the other channels. Additionally, although our discussion has dealt only with statistical error, naively combining the channels in this manner would not account for the different systematic errots the individual processes would have.

We note that, even the bounds listed above for the individual processes, are approarhing the few percent realm for $\Delta \kappa_{V}$, the scale of deviation predicted by "new physics" theories. The limits at $1 \mathrm{TeV}$ are particularly interesting because they are of the same order as the standard model radiative corrections.

\subsubsection{Effect of Including Systematic Uncertainties}

The limits for $\Delta \kappa_{V}$ were extracted from the statistical $\pm 2 \sigma$ contour lines and did not account for any systematic uncertainty in the cross-section measurement. Such systematic error is of course unavoidable in a real experimental situation, it comes from uncertainties in luminowity determination, background subtraction, detector acceptance, and other experimental limitations. If we make rough estimates of the uncertainties for the above factors of $\delta L \sim 1 \%, \delta_{\text {back }} \sim 1 \%$, and $\delta_{\text {det }} \sim 1 \%$ [38], then we obtain an overall systematic uncertainty of $\sim 2 \%$. The $2 \sigma$ statistical uncertainty goes like $2 \sqrt{N} / N$, or equivalently, $2 / \sqrt{\sigma L}$, where $\sigma$ is the cross-section. 'Thus, assuming an integrated luminosity of $\mathrm{L}=50 \mathrm{fb}^{-1}$, and for cross-sections of the order $\sigma \sim 0.2 \mathrm{pb}$, the systematic uncertainty is comparable to that from the statistics. For smaller crows-sections, the statistical error will dominate over the systematic. At $\sqrt{8}=500 \mathrm{GeV}$, the Standard Model cross-sections ranged from $0.034 \mathrm{pb}\left(\mu^{+} \tau^{-}\right)$to $0.1 \mathrm{pb}\left(e^{+} e^{-}\right)$, so it seems that we border on the scale at which systematic uncertainty might become comparable to that from the statistics. 
A rross-section of $0.1 \mathrm{pb}$ produces a statistical uncertainty of $\sim 2.8 \%$. Not accounting for the systematic error of $\sim 2 \%$ would therefore underestimate the overall cross-section uncertainty of $\sim 3.4 \%$. With the uncertainty in $\sigma$ underestimated, the resultant limits on $\Delta \kappa_{V}$ will be overestimated, $\kappa_{\gamma}$ and $\kappa_{z}$ unrealistically constrained. However, the sensitivity to $\Delta \kappa_{V}$ is such that loosening the error on $\sigma$ from $2.8 \%$ to $3.4 \%$ will not significantly alter the resultant $\kappa V$ limits, eg. they loosen from $\sim \pm 10 \%$ to $\sim \pm 11 \%$. For the other channels, and at the higher energy of $\sqrt{s}=1 \mathrm{TeV}$, the cross-sections are smaller than $0.1 \mathrm{pb}$, and so the statistical uncertainty can be assumed to dominate the systematic error. Overall then, accounting for systematic uncertainties from realistic experimental limitations would loosen the determined bounds on $\kappa_{V}$, but not to a significant extent.

\subsubsection{Scaling Behaviour of Sensitivities}

We have seen that the sensitivity to the non-standard couplings is improved with higher energy, this is a consequence of the increasing non-standard cross-section at asymptotic energies. The . discrepancy between the Standard Model cross-section $\sigma_{S M}$ and the non-standard $\sigma$, and the resultant sensitivity to the couplings, thus grows with increasing $\sqrt{s}$. We can derive a simple relation approximating this energy dependence of the $\kappa_{V}$ sensitivity, temporarily ignoring differences between $\kappa_{\gamma}$ and $\kappa_{z}$. We saw previously that the functional dependence of the cross-sections on $\Delta$ niv was parabolic, see Figures (4.4) and (4.5). Therefore, we write for the non-standard ross-section

$$
\sigma=a(s)+b(s) \Delta \kappa v+c(s)(\Delta \kappa v)^{2}
$$

where $a(s), b(s)$, and $c(s)$ are energy dependent factors.

The discrepancy between this cross-section and that of the Standard Model, $\sigma_{S M}=a(s)$, is then

$$
\begin{aligned}
& \Delta \sigma=\sigma-\sigma_{S M} \\
& \Delta \sigma=b(s) \Delta \kappa_{V}+c(s)(\Delta \kappa V)^{2}
\end{aligned}
$$

The number of non-standard events, excess to those of the Standard Model,is then

$$
\Delta N=L\left(b(s) \Delta \kappa_{V}+c(s)(\Delta \kappa V)^{2}\right)
$$


These excess events will only be significant if their number exceeds the statistical error on the number of events expected from the standard cross-section. which we denote as $\delta . N$. The critical relation is therefore

$$
\begin{aligned}
\Delta N & =\delta N \\
L\left(b(s) \Delta \kappa_{v}+c(s)\left(\Delta \kappa_{v}\right)^{2}\right) & =\sqrt{L \sigma_{s M}} \\
\sqrt{\frac{L}{\sigma_{S M}}\left(b(s) \Delta \kappa_{V}+c(s)\left(\Delta \kappa_{V}\right)^{2}\right)} & =1
\end{aligned}
$$

The rough symmetry of the surface plots, Figures (4.4) and (4.5), and the contour plots, Figures (4.6) and (4.7), about the origin $\left(\Delta \kappa_{\gamma}=0, \Delta \kappa_{z}=0\right)$ at $\sqrt{s}=1 \mathrm{TeV}$ impiies that. the quadratic term in the non-standard cross-sections dominates at asymptotic energies, and $c(s)>b(s)$ for $s>>M_{2}^{2}$. The approximate high energy behaviour of the nonstandard crosssections is to scale with energy such that $c(s) \approx s$, as can be verified from a plot of $\sigma$ vs. $s$ on a linear scale. Also, the standard cross-section $\sigma_{S M}$ scales like $\ln s / s \sim 1 / s$ (this behaviour is verified by the Standard Model cross-sections of $0.034 \mathrm{pb}$ and $0.009 \mathrm{pb}$ for the $\mu^{+} \tau^{-}$, whannel at $\sqrt{s}=500 \mathrm{GeV}$ and $1 \mathrm{TeV}$ respectively). We therefore find the following relation

$$
\begin{aligned}
\sqrt{L s} \cdot s \cdot\left((\Delta \kappa V)^{2}\right) & =1 \\
s^{3 / 2} \sqrt{L} & =\frac{1}{(\Delta \kappa v)^{2}} \\
\left(s^{3} L\right)^{1 / 4} & =\frac{1}{(\Delta \kappa V)}
\end{aligned}
$$

The significance of this expression is that as $\sqrt{5}$ and $L$ increase, the minimum significantly determinable $\Delta \kappa_{v}$ decreases. If we define the sensitivity for the measurement of $\Delta \boldsymbol{k}_{v}$ as the inverse of the magnitude of $\Delta \kappa v$. Eq. (4.4) implies that the sensitivity scales approximately as $\left(s^{3} L\right)^{1 / 4}$. This simple relation gives quite good agreement with the explicitly determined sensitivities of Table 4.1. For instance, doubling the energy from $\sqrt{s}=500$ GeV to $1 \mathrm{TeV}$, we expect to improve our sensitivity to $\Delta \kappa_{V}$ by a factor $\left(2^{6}\right)^{1 / 4} \sim 2.8$. By examining the limits of Tr.sle 4.1, we see that the sensitivities increased on average by a factor of 2.6 in going to the higher energy.

In general, the sensitivity relation, Eq. (4.4), tended to underestimate the improvernent in sensitivity for the simpler proccesses, eg. $\mu^{+} \tau^{-}$, and overestimate for the more complicated, such as $e^{+} e^{-}$. Overall though, it was able to reasonably approximate the explicit numerical 
dependence of the $\Delta \kappa_{V}$ sensitivity on energy. Similarly, the dependence of the $\kappa_{V}$-sensitivity on luminosity was examined. For instance, doubling luminosity improves the percentage statistical error by a factor of $\sqrt{2}$, evidenced by a smaller $2 / \sqrt{L \sigma_{S M}}$. The limits on $\Delta \kappa_{V}$ however, are determined from the intersection of this improved $95 \%$ C.L. cross-section with the $\Delta \kappa v$ dependent parabola. Effectively then, improving $2 / \sqrt{L \sigma_{S M}}$ by a factor $\sqrt{2}$ will improve the resultant limit on $\Delta \kappa_{V}$ by a factor of $2^{1 / 4}$, as predicted. The $L^{1 / 4}$ dependence for the sensitivity, as opposed to the more intuitive $L^{1 / 2}$ (as determined by Bilenky et al. [62], in their analysis of scaling behaviour for $e^{+} e^{-} \rightarrow W^{+} W^{-}$), is thus seen to be a consequence of the dominance of the quadratic term $c(s)\left(\Delta \kappa_{V}\right)^{2}$ to the anomalous cross-section. If the linear term was assumed dominant, then the expected scaling behaviour of the $\kappa_{V}$ sensitivity would be $\sqrt{s^{3} L}$. This however gives poor agreement with the explicitly determined limits of Table 4.1).

In principle, once the sensitivity to $\Delta \kappa_{v}$ is known for a given combination of energy and luminosity, Eq. (4.4) could be used to roughly predict the sensitivity for any other pairings of $s$ and $L$. It can also give estimates of the required scales for energy and luminosity necessary to achieve a specified level of sensitivity.

\subsection{Distributions as Indicators of Non-Standard Vertex Couplings}

In addition to total sross-section values, we generated a number of distributions, both those that would be experimentally observable, and others, used to check the consistency of our results. We discuss these checks in detail in Appendix E. The experimentally observable distributions studied include the differential cross-section with respect to the opening angle between the charged leptons,$\theta_{+-}$, the angle of each charged lepton with the incoming $e^{-}, \theta_{l+}$ and $\theta_{l-}$, the energy, $E_{l}$, and transverse momentum, $p_{\boldsymbol{r}}^{f}$, of each charged lepton, the total visible energy, $E_{\mathrm{vis}}$, and transverse momentum, $P_{T}$, and the invariant mass of the charged lepton pair, $M_{t+t^{\prime}-\text {. }}$

We show in Figure (4.8) the distributions for the three angular variables, $\theta_{+-}, \theta_{1+}$, and $\theta_{1-}$, respectively, for the $\mu^{+} r^{-}$process at $\sqrt{s}=500 \mathrm{GeV}$. For each plot, the solid line corresponds to the Standard Model values, the dashed line to $\kappa_{\gamma}=\kappa_{Z}=0.9$, and the dotted line to $\kappa_{\gamma}=\kappa_{Z}=$ 1.1 . 

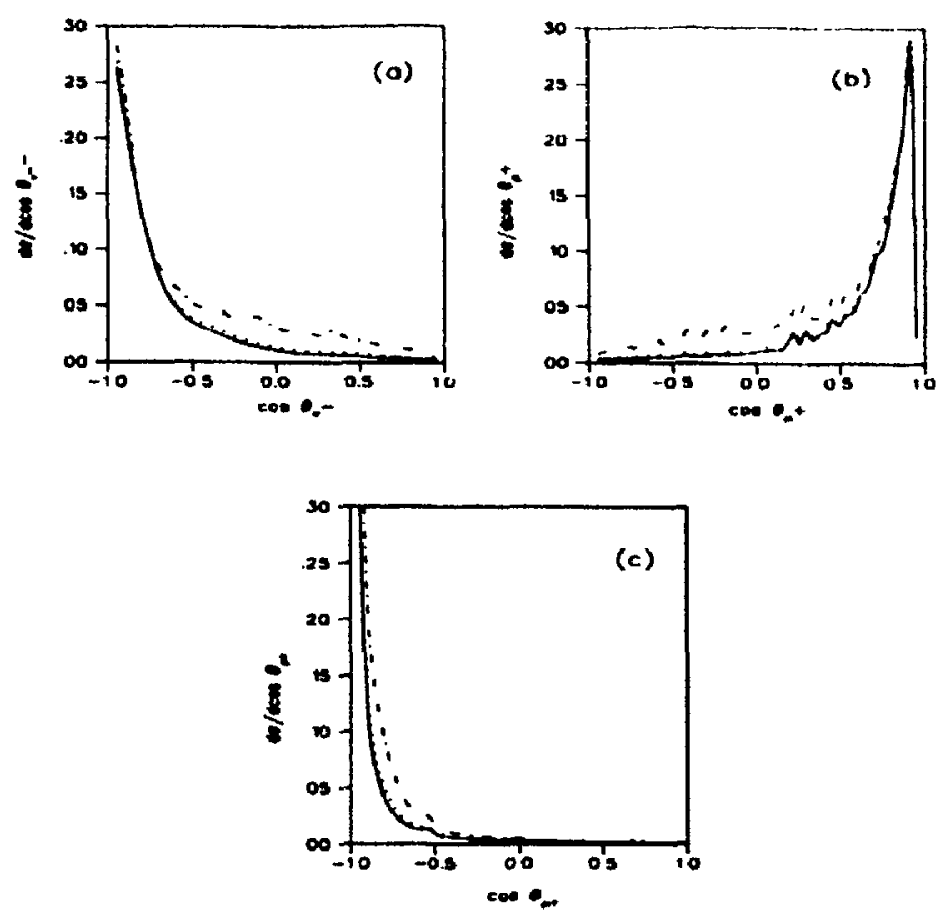

Figure 4.8: Differential cross-sections with respect to (a) $\cos \theta_{r_{-}}$, (b) $\cos \theta_{\mu^{+}}$, and (c) $\cos \theta_{\mu \tau}$ for Standard Model(solid line), $\Delta \kappa_{\gamma}=\Delta \kappa_{z}=-0.1$ (dash-dotted line), and $\Delta \kappa_{\gamma}=\Delta \kappa_{z}=$ 0.1 (dotted line).

The strong peaks in the individual beam angle distributions, and consequently in that of the opening angle between them, are due to the t-channel neutrino exchange diagram of $W$ pair production, Figure (3.2a). The neutrino propagator for these diagrams has the form, neglerting lepton masses,

$$
\frac{1}{(k-p)^{2}}=-\frac{1}{-2 E_{k} E_{p}\left(1-\cos \theta_{k p}\right)}
$$

where $k$ is the momentum of the incoming electron(positron) and $p$ the momentum of its like sign outgoing charged $W$. The propagator pole drives the $W$ 's to be emitted in the direction of thrir same sign initial $e^{+}$or $e^{-}$, such that $\cos \theta_{k p} \simeq 1$. The $W$ decay charged leptons then get boosted in the same direction as their parents, and so are preferentially emitted along the beasi line. We: define the positive beam direction as that of the incoming positron, and so the positively c:targerl lepton's distribution peaks at $\cos \theta_{k p} \simeq 1$ and that of the negatively charged at $\cos \theta_{k p} \simeq-1$. This behaviour is repeated for the other three processes, but is less marked. There, the many 
extra diagrams dilute the effect of the t-channel neutrino diagram.

For non-standard pairings of $\kappa_{\gamma}$ and $\kappa_{\Sigma}$, these angular distributions are generally somewhat rnhanced in the regions of phase space away from these peaks. This is understandable; the tchannel neutrino diagram does not contain the triple boson vertex, and the peak therefore should be relatively insensitive to the vertex couplings. We will explore in the next Section the possibility of excluding through cuts the large $\kappa$ independent peak regions to improve our sensitivity to $\kappa v$.

We saw from the total cross-sections of the previous section that, generally, non-standard pairings with $\Delta x>0$ show less deviation than those with $\Delta x<0$. The curves of Figure (4.8) of course exhibit the same behaviour; the case of $\kappa_{\gamma}=\kappa_{z}=0.9$ shows a greater deviation from the SM than does $\kappa_{\gamma}=\kappa_{Z}=1.1$, and this pattern holds true over the whole phase space. The extra $\kappa$ dependent diagrams that contribute to the $\mu^{+} e^{-}$and $e^{+} e^{-}$processes complicate the situation. For these processes, there are regions of the angular phase space in which the $\Delta x>0$ case is more sensitive than $\Delta x<0$. For example, in Figure (4.9), part of the distribution in the angle $\theta_{e}-$, for the $\mu^{+} e^{-}$process at $500 \mathrm{GeV}$ is shown. Here we concentrate on the peak region of the full phase space, restricting phase space to $-0.85<\cos \theta_{e}<-0.75$. The three curves are those of the SM, $\kappa_{\gamma}=\kappa_{Z}=0.9$, and $\kappa_{\gamma}=\kappa_{Z}=1.1$.

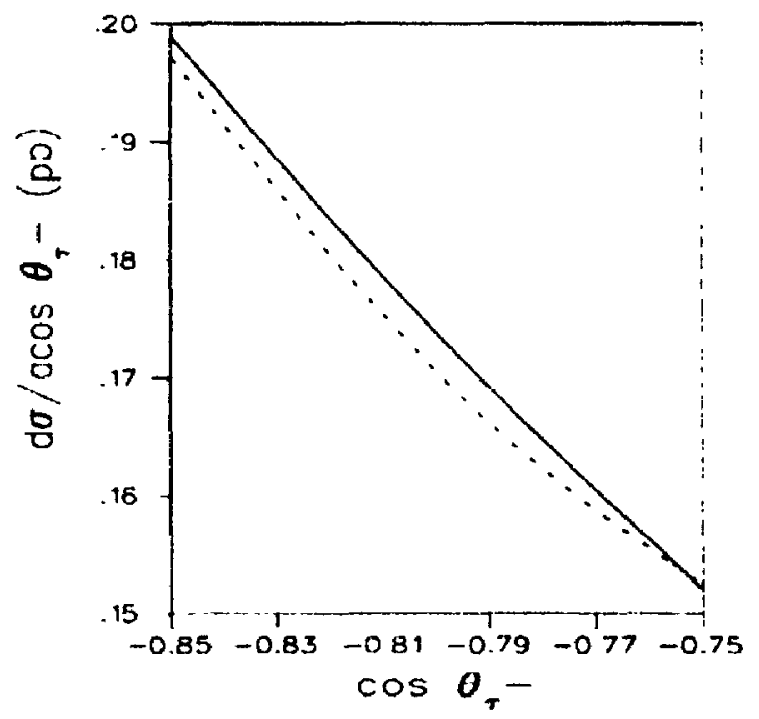

Figure 4.9: Differential cross-section with respect to $\cos \theta_{e}$ for the $\mu^{+} e^{-}$channel at $\sqrt{s}=500$ GeV. The curves correspond to Standard Model (solid line), and non-standard $\kappa_{\gamma}=\kappa_{Z}=0.9$ (dash-dotted line), and $\kappa_{\gamma}=\kappa_{Z}=1.1$ (dotted line). 
It is apparent that, over this small portion of the full phase space, the $\mu^{+} e^{-}$provess is mowt sensitive to variations where $J_{\kappa}>0$ although. upon integrating over the full angular range we find greater sensitivity to $\Delta x<0$. In addition, over this limited phase spater region. a deviation $\Delta x<0$ would manifest itself in a below standard integrated cross-section: this is the oppewile of what occurs when $\Delta \kappa>0$. Such a cross-section is a signature of the interference elfects betwern the standard and non-standard amplitudes; the non-standard contributions, taken on their own, can only increase the total cross-section.

The individual energy and transverse momentum distributions are generally 'mbamcerl quile evenly over their range. We show in Figure (4.10) the differential cross-ser'tion with respent to the normalized energy of the negatively charged lepton, $x_{-}$, for the $\mu^{+} T^{-}$pror(sis at. $\left.\sqrt{s}=500\right)$ $\mathrm{GeV}$. The solid line corresponds to the standard model, the dash-dotted line to $\kappa_{\mathrm{\gamma}}=\kappa \%=0.9$. and the dotted line to $\kappa_{\gamma}=\kappa_{Z}=1.1$.

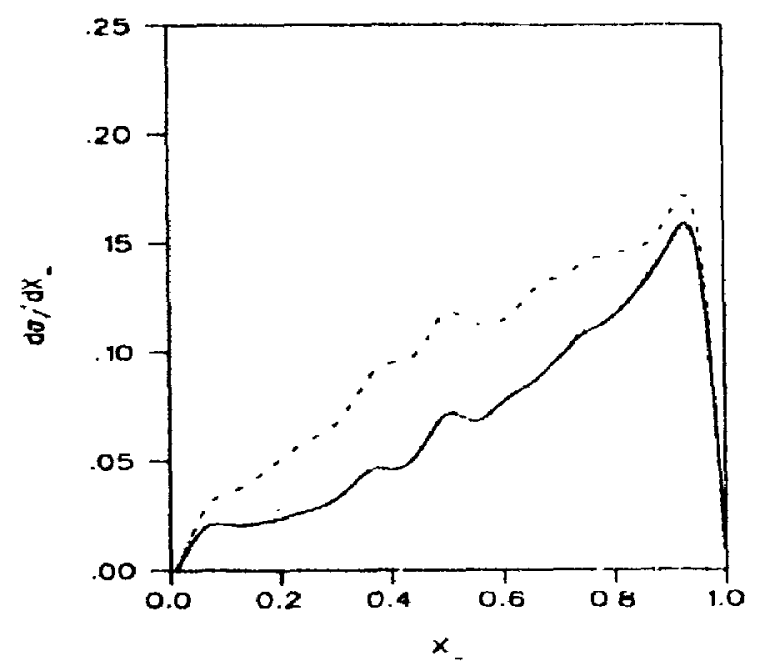

Figure 4.10: Differential cross-section with respect to $x_{-}$, the energy of the outgoing leptron, normalized to that of the incoming $e^{-}$, for Standard Model (solid line), and non-standard $\kappa_{\gamma}=\kappa_{Z}=0.9$ (dash-dotted line), $\kappa_{\gamma}=\kappa_{Z}=1.1$ (dotted line).

The distributions in the invariant mass end total energy of the outgoing lepton-antilepten pair demonstrate the same type of behaviour, the non-standard couplings can product qualitative its well as quantitative changes. Figures (4.11) and (4.12) are the distributions in the charged leptron pair invariant mass and total energy for the $\mu^{+} \tau^{-}$process at $\sqrt{5}=500$ GeV. 'The nort-staurlard values of $\kappa_{\gamma}=\kappa_{Z}=0.9$ produce a markedly different overall appearance in the differrutual 
cross-section than that obtained for the standard model values.

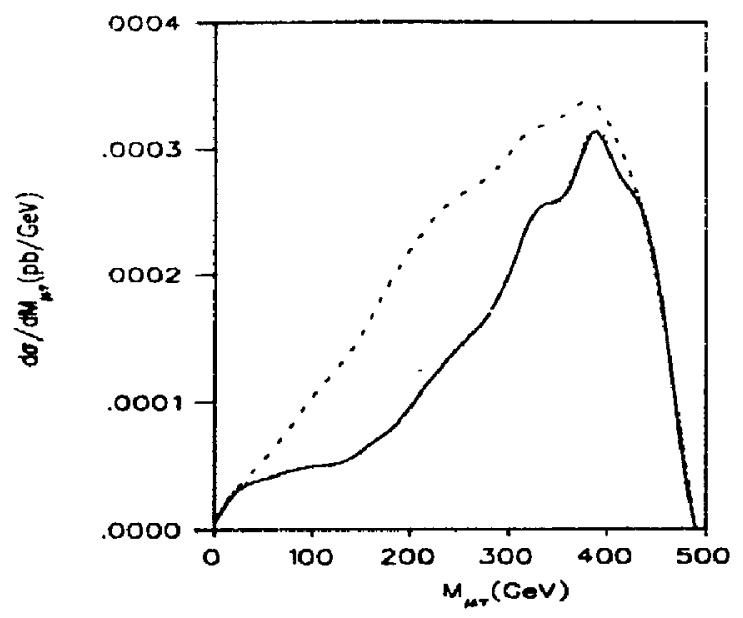

Figure 4.11: Differential cross-section with respert to $M_{\mu \tau}$, the invariant mass of the $\mu^{+} \tau^{-}$. pair, for Standard Model(solid line), and non-standard $\kappa_{\gamma}=\kappa_{Z}=0.9$ (dash-dotted line), and $\kappa_{\gamma}=\kappa_{Z}=1.1$ (dotted line).

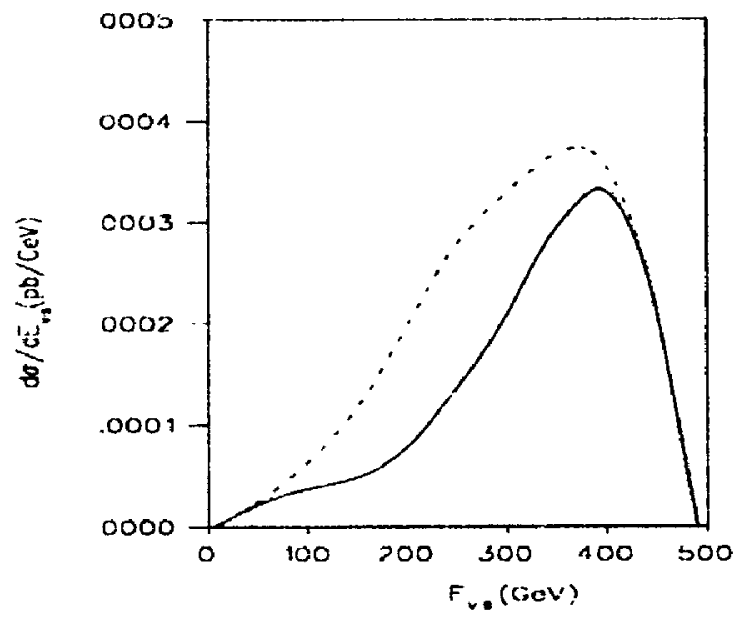

Figure 4.12: Differential cross-section with respect to $E_{v i s}$, the total energy of the $\mu^{+} \tau^{-}$pair, for Standard Model,(solid line), and non-standard $\kappa_{\gamma}=\kappa_{Z}=0.9$,(dash-dotted line), and $\kappa_{\gamma}=$ $\kappa_{Z}=1.1$ (dotted line). 
It is apparent from Figures (4.11) and (4.12) that there are significant contributions to the total cross-sectiou from regions. $M_{\mu \mathrm{r}}, E_{\mu \tau} \leq 100 \mathrm{GeV}$ and $M_{\mu \mathrm{r}}, E_{\mu \mathrm{T}} \geq 400 \mathrm{GeV}$, with very little $\kappa$ dependence. Such behaviour suggests the possibility of maximizing the sensitivity to the vertex couplings by excluding these non-dependent phase space regions through the implementation of suitable experimental cuts. Such a cut would be experimentally viable as the variables $M_{\mu r}$ and $\Sigma_{\mu \tau}$ have no dependence on the final state neutrinos. We explore this possibility in the following Section.

The irivariant mass distribution of the two muons in the $\mu^{+} \mu^{-}$process motivates another possible invesiigation; we show this distribution at the center of mass energy of 500 (irV in Figure (4.13).

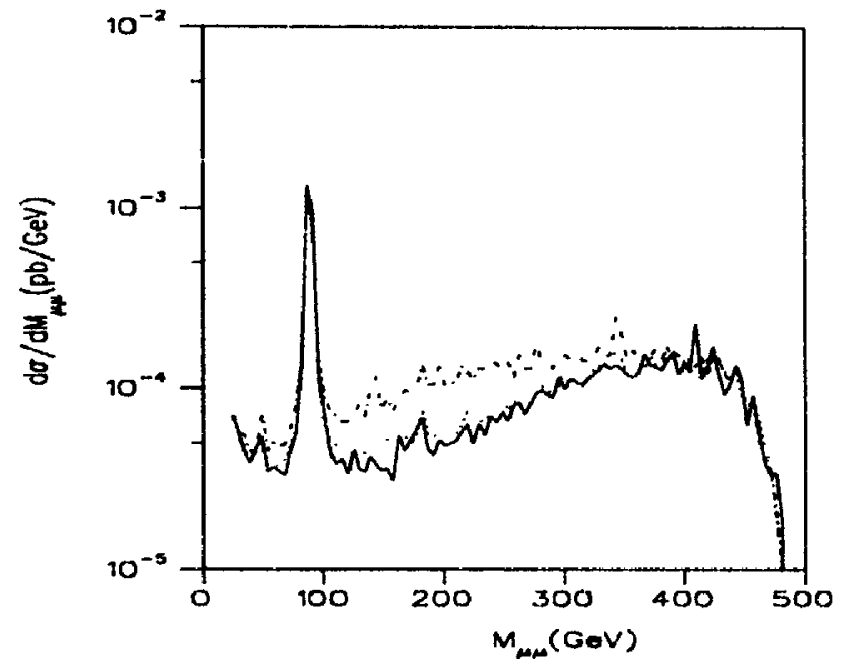

Figure 4.13: Differential cross-section with respect to $M_{\mu \mu}$, the invariant mass of the $\mu^{+} \mu^{-}$pair at $\sqrt{S}=500 \mathrm{GeV}$ for Standard Model(solid line) , and non-standard $\kappa_{\gamma}=\kappa_{z}=0.9$ (dash-dotted line), $\kappa_{\gamma}=\kappa_{Z}=1.1$ (dotted line).

The strong peak at the $\mathrm{Z}$ pole, $M_{\mu \mu}=M_{Z}$, part of which is from the $\kappa$-dependent contribution of Figure (3.5), the t-channel fusion of two $W$ bosons to a neutral boson $\gamma$ or $Z$, suggests the possibility of cutting on the invariant mass of the $\mu^{+} \mu^{-}$pair to isolate the $Z W W$ vertex, and explore the sensitivity to the coupling $\kappa_{z}$, independent of $\kappa_{\gamma}$. This possibility was discussed hy Godfrey et al. [33]. We will also explore this idea in Section 4.4.

The total visible transverse momentum distribution exhibits its greatest deviation from the standard model in the regions of phase space where the differential cross-section is itself Jargest. 
We illustrate this in Figure (4.14) for the $\mu^{+} \mu^{-}$channels at $\sqrt{s}=500 \mathrm{GeV}$.

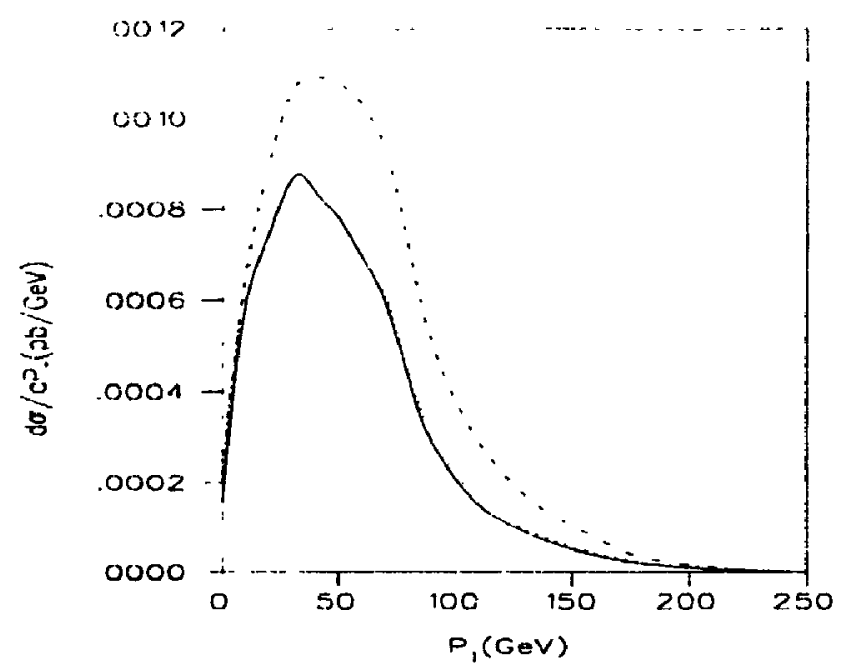

Figure 4.14: Differential cross-section with respect to $P_{T}$, the total visible transverse momentum, for Standard Model(solid line), and non-standard $\kappa_{\gamma}=\kappa_{Z}=0.9$ (dash-dotted line), $\kappa_{\gamma}=\kappa_{Z}=$ 1.1(dotted line) for the $\mu^{+} \mu^{--}$channels at $500 \mathrm{GeV}$

The various differential cross-sections contain a great deal of information, beyond that of merely integrating over them to obtain the total cross-sections. Because of the qualitative, and not just quantitative, changes in their form that occur when non-standard couplings are introduced, some distributions offer a more direct indicator of such couplings, additional to total cross-section measurements. As we have indicated, the "localization" of sensitivity to $\kappa$ to certain regions of phase space offers the prospect of cutting on certain experimental variables to isolate these sensitive regions, and in doing so, improve the limits on $\kappa_{V}$.

\subsection{Improving Sensitivity Through Cuts}

By examining the various distributions, it is apparent that the sensitivity to non-standard $\kappa v$ is not always uniform over the phase space range of a particular variable. For instance, we saw in the previous Section that the distributions in the angular variables $\theta_{l+}$ and $\theta_{l-}$, (the anti-lepton and lepton beam line angles respectively, defined with respect to the incoming electron), show little $\mathrm{nV}_{\mathrm{V}}$ sensitivity in the strong peaks at $\cos \theta_{l^{+}}=1$ and $\cos \theta_{l^{-}}=-1$, Figures (4.8a) and (4.8b). For the $\mu^{+} \tau^{-}$channel, these peaks are due to the $\kappa$-independent t-channel neutrino exchange 
diagram, Figure (3.2a); with consequently little sensitivity to anomalous vertex couplings in the peaks.

This localization of the $\kappa$ sensitivity to the "non-peak" regions of the $\theta_{l+}$ and $\theta_{l}$ - phase spare suggests the potential for maximizing the $\kappa$ sensitivity by cutting on these variables. laking the simplest $\mu^{+} \nu_{\mu} \tau^{-} \bar{\nu}_{T}$ process as an example, by requiring that the angular variables satisfy

$$
\begin{aligned}
& \cos \theta_{\tau-}>-0.9 \\
& \cos \theta_{\mu}+<+0.9
\end{aligned}
$$

we would effectively exclude the large non-sensitive peak contributions, and isolate the $n$ dependent plateau regions. Because, by making such a cut, we lose a significant portion of our total cross-section, we must distinguish between improving the physical sensitivity, as a \% deviation from the SM total cross-section, and the experimental sensitivity, defined as the potential for detection of anomalous couplings. We will see that increasing the physical $\kappa v$ sensitivity through cuts does not guarantee an improved limit on the parameters; the decrease in cross-section can be statistically prohibitive.

To simplify matters, we restrict our study to the case $\kappa_{\gamma}=\kappa_{Z}$. Any limits derived with the angular cuts in place must therefore be compared to the corresponding diagonal limits from the contour plots of Figures 4.6 and 4.7. Although more constrained than those obtained by making no assumptions about the relationship between $\kappa_{\gamma}$ and $n_{z}$, comparing these limits will satisfactorily demonstrate the scale of the improvements in sensitivity possible.

We define a cut parameter $\theta_{C}$

$$
\begin{aligned}
& \cos \theta_{\tau^{-}}>-\theta_{C} \\
& \cos \theta_{\mu^{+}}<+\theta_{C}
\end{aligned}
$$

Experimental beam-pipe detection limitations motivated our "weak" cut of $\theta_{C}=0.95$; w" here consider making "stronger" cuts, $\left|\theta_{C}\right|<0.95$. Choice of a cutoff $\theta_{C}$ is a compromise: between completely excluding the non-sensitive peak and maximizing cross-section; the first requirement of peak exclusion suggests a cutoff more extreme than that desirable for the second requirement of maximum cross-section. In Table 4.2, for the $\mu \tau\left(\mu^{+} \tau^{-}\right.$and $\left.\mu^{-} \tau^{+}\right)$channel at $\sqrt{s}=500 \mathrm{GeV}$, we show the cross-sections for $\kappa_{\gamma}=\kappa_{Z}=1.0,0.9$ and 1.1 for the three cases of angular cuts $\theta_{C}=0.95, \theta_{C}=0.9$, and $\theta_{C}=0.7$. The choices of $\theta_{C}=0.95$ and $\theta_{C:}=0.9$ 
wrre taken as representing the extremes of optimizing the two opposing requirements. $\theta_{C}=0.9$ maximizes statistics and $\theta_{C}=0.7$ excludes essentially all of the peak contribution. The ratio $R$ of thon-standard cross-section to $\sigma_{S M}, R=\sigma / \sigma_{S M}$, is listed to allow comparison of the physical sensitivity for the three cases. The cross-sections have been multiplied by 2 to account for the charge conjugate process.

Table 4.2. Cross-sections and ratios relative to the SM for different angular cuts for $\mu \tau$ process at $500 \mathrm{GeV}$.

\begin{tabular}{|l|ll|ll|ll|}
\hline & \multicolumn{2}{|c|}{$\theta_{C}=0.95$} & \multicolumn{2}{c|}{$\theta_{C}=0.9$} & \multicolumn{2}{c|}{$\theta_{C}=0.7$} \\
\hline$\kappa_{\gamma}=\kappa_{Z}$ & $\sigma(\mathrm{pb})$ & $\mathbf{R}$ & $\sigma(\mathrm{pb})$ & $\mathbf{R}$ & $\sigma(\mathrm{pb})$ & $\mathbf{R}$ \\
\hline 0.9 & 0.097 & 1.41 & 0.064 & 1.60 & 0.039 & 2.30 \\
1.0 & 0.069 & 1.00 & 0.040 & 1.00 & 0.017 & 1.00 \\
1.1 & 0.073 & 1.06 & 0.043 & 1.08 & 0.020 & 1.18 \\
\hline
\end{tabular}

The decrease in total cross-section for the strong angle cuts is apparent, as is the improved physical sensitivity, indicated by the larger values for the ratio $R$ with the stronger cuts implemented. To compare the achievable experimental bounds for the different cuts, we assume an integrated luminosity of $50 \mathrm{fb}^{-1}$ and determine the $2 \sigma$ confidence interval about the expected number of events $N$. We list these $2 \sigma$ limits in Table 4.3.

Table 4.3: $2 \sigma$ limits on $\Delta \kappa v$ for various angular cuts for $\mu \tau$ process at $500 \mathrm{GeV}$.

\begin{tabular}{|l|l|l|}
\hline Angular Cut & $\Delta \kappa<0$ & $\Delta \kappa>0$ \\
\hline$\theta_{C}=0.95$ & $1.5 \%$ & $9.3 \%$ \\
$\theta_{C}=0.9$ & $1.2 \%$ & $9.3 \%$ \\
$\theta_{C}=0.7$ & $0.8 \%$ & $9.0 \%$ \\
\hline
\end{tabular}

We see that making a cut on the angular variables can significantly improve the limits for $\Delta \kappa<0$, but has little effect for $\Delta \kappa>0$. Also, the more restrictive cut of $\theta_{C}=0.7$, despite a sizable decrease in cross-section, gives a tighter constraint on $\Delta \kappa$ than does the choice of $\theta_{C}=0.9$. It seems that the improvement in physical sensitivity more than compensates for the loss of statistics. 
While the peak regions of the angular distributions for the $\mu^{+} \tau^{-}$process are, for the most part. insensitive to the triple boson vertices, these same regions can have ni dependence for the $\mu^{+} e^{-}$and $e^{+} e^{-}$channels. For these two final state configurations, the t-channel TuV dependent. diagrams of Figure (3.3), gives a $n$-dependent contribution to these regions. 'lhus, for the $\mu^{+} .^{-}$ and $e^{+} e^{-}$channels, a cut to exclude the peak regions can result in a loss of both statistics and sensitivity, with consequently weaker bounds on anomalous couplings. Because this t-channel diagram shows greatest sensitivity to deviations $\Delta n_{V}>0$; it is the limits on such deviations that are loosened by such a cut. The limits on deviations $\Delta \alpha_{V}<0$, because the sensitivity to such anomalous couplings is not concentrated in the peak regions can, however, be intproved. For instance, for the $\mu e$ channel at $\sqrt{s}=500 \mathrm{GeV}$, we compare $2 \sigma$ linits for the two cases of $\theta_{C}=0.95$ and $\theta_{C}=0.7$.

$$
\begin{gathered}
\theta_{C}=0.95-2.7 \%<\Delta \kappa_{V}<+7.0 \% \\
\theta_{C}=0.7-1.1 \%<\Delta \kappa_{V}<+8.0 \%
\end{gathered}
$$

The angular variables are not unique in their localization of sensitivity to nv. Another experimental observable is $M_{l+l^{\prime}}$, the invariant mass of the outgoing lepton-antilepton pair. Figure (4.11) illustrated how the sensitivity to non-standard $\kappa v$ is located predominantly in the middle region of the phase space for the $\mu^{+} r^{-}$process at $\sqrt{s}=500 \mathrm{GeV}$. A cut

$$
30<M_{\mu \tau}<430
$$

would exclude the $\kappa$-insensitive contributions from the extreme low and high invariant mass regions of phase space. We have found that such a cut, on its own, is unable to significantly improve the limits on $\kappa_{V}$, the improved physical sensitivity is insufficient to account for the loss of statistics. For instance, with the above cut in place, the achievable limits on $\kappa_{V}$ for the $\mu^{+} \tau^{-}$ channel at $\sqrt{8}=500 \mathrm{GeV}$ are

$$
-2.4 \%<\Delta \kappa v<7.5 \%
$$

which are not significantly improved relative to the "no-cut" case for $\Delta \kappa_{v}<0$ and slightly loosened for $\Delta \kappa v>0$. We can, however, consider making combined cuts, both an angular and invariant mass cut. Figure (4.15) shows the invariant mass distribution for the $\mu^{+} T^{-}$pair $^{\text {ut }}$ $\sqrt{s}=500 \mathrm{GeV}$ for the two cases of $\theta_{C}=0.95$, and $\theta_{C}=0.7$. The solid line corresponds to the Standard Model case; the dotted line to $\kappa_{\gamma}=\kappa_{Z}=0.9$. 

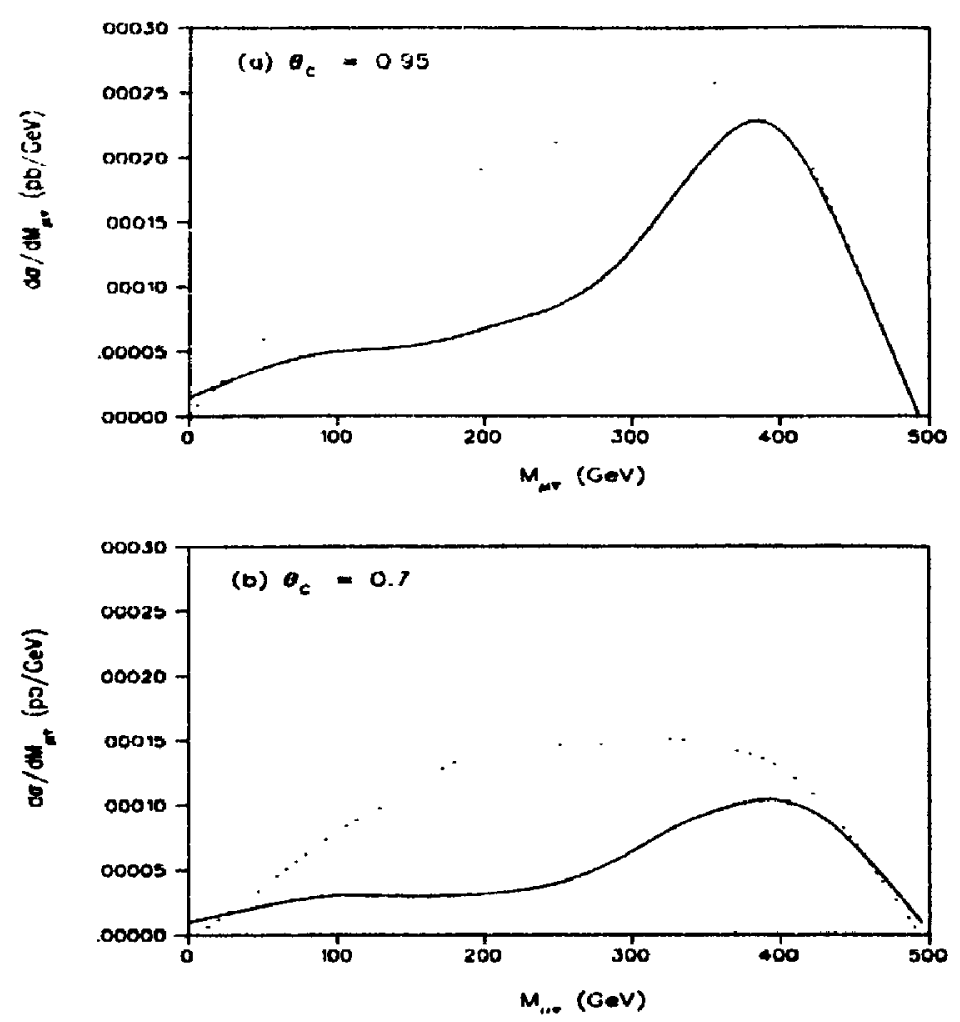

Figure 4.15: Distributions in invariant mass $M_{\mu r}$ for $\mu^{+} \tau^{-}$channel at $\sqrt{s}=500 \mathrm{GeV}$ for (a) $\theta_{C}=0.95$ and (b) $\theta_{C}=0.7$. For both plots, the solid line corresponds to $\kappa_{\gamma}=\kappa_{Z}=1.0$ and the dotted line to $\kappa_{\gamma}=\kappa_{z}=0.9$.

It seems that the tighter angular cut somewhat exaggerates the tendency of the $\kappa$ sensitivity to be localized in the middle region of the invariant mass phase space; thereby making an invariant mass cut more effective at isolating these regions. We therefore investigated various combinations of angular cuts $\theta_{C}$ and invariant mass cuts. We list in Table 4.4 the $2 \sigma$ limits for certain combinations of cuts. 
Table 4.4: $2 \sigma$ limits for various cut combinations for $\mu^{+} \tau^{-}$process at $\sqrt{\mathrm{s}}=500(\mathrm{ieV}$.

\begin{tabular}{|l|l|l|}
\hline Cuts & $\Delta_{\kappa}<0$ & $\Delta_{\kappa}>0$ \\
\hline$\theta_{C}=0.95$ & $1.5 \%$ & $9.3 \%$ \\
$\theta_{C}=0.9$ & $1.2 \%$ & $9.3 \%$ \\
$\theta_{C}=0.7$ & $0.8 \%$ & $9.0 \%$ \\
$\theta_{C}=0.7,30<M_{\mu r}<430$ & $1.0 \%$ & $8.5 \%$ \\
$\theta_{C}=0.7,100<M_{\mu r}<350$ & $0.79 \%$ & $9.0 \%$ \\
\hline
\end{tabular}

The tightest limits are obtained with cuts chosen as follows; $\theta_{c}=0.7$ implemented alone, and in combination with the invariant mass cut, $100<M_{\mu r}<350$. The bounds on $\Delta x<0$ can be improved by almost a factor of two relative to the "weak" angle cut: the bounds for $\Delta x>0$ are not significantly improved. Both these cuts were chosen as optimizing the physical sensitivity at the cost of loss of total cross-section, as opposed to the other extreme of maximizing crosssection by including regions of lower physical sensitivity. Such seemingly overly restrirtive cuts, excluding more of phase space than is perhaps indicated by the shape of the diatributions, are thus suggested for optimizing the bounds on anomalous couplings.

The previous limits are "diagonal", that is, they were determined on the assumption that $\kappa_{\gamma}=\kappa_{Z}$. If we relax this requirement, and do a complete $2 \sigma$ contour analysis, as in the previous Section, we can compare the more general limits obtained for the two couplings. We repeat the full contour analysis for the case of the combined cuts, $\theta_{C}=0.7$ and $100<M_{\mu r}<350$. We extract limits for $\kappa_{\gamma}$ and $\kappa_{Z}$ from the $\pm 2 \sigma$ curves and list them below. For comparison, we: show also the limits derived from the $2 \sigma$ contour for the case of no sensitivity enhancing cut, as listed in Table (4.1). 


$$
\begin{gathered}
\theta_{C}=0.7,100<M_{\mu r}<350 \\
0.98>\kappa_{\gamma}, \quad 1.01<\kappa_{\gamma}<1.1, \quad \kappa_{\gamma}>1.13 \\
0.95>\kappa_{z}, 0.98<\kappa_{z}<1.07, \quad \kappa_{z}>1.09 \\
\text { No Cuts } \\
0.975>\kappa_{\gamma}>1.15 \\
0.94>\kappa_{z}>1.1
\end{gathered}
$$

The limite are seen to be significantly improved. The improvements, for the most part, are a consequence of the annular shape determined by the $\pm 2 \sigma$ contours for the "cut" case. The $-2 \sigma$ contour was not relevant (being below the parabola minimum) for the "no-cut" case, and the disc defined by the $+2 \sigma$ contour is far less constraining.

As was described in Chapter 4, the individual energy and transverse momentum distributions, unlike the angular and invariant mass distributions, are generally enhanced evenly over most of their phase space. These distributions therefore do not provide a clear opportunity for optimizing the sensitivity through simple cuts.

The previous investigations have been at the center-of-mass energy of $500 \mathrm{GeV}$. The sensitivity to $\kappa$ is generally more evenly distributed over the available phase space at the higher energy of $1 \mathrm{TeV}$. For instance, although the effect of the neutrino propagator in driving the $\kappa_{V}$-insensitive diagram of Figure (3.2a) to contribute to the angular regions $\cos \theta_{l \pm}= \pm 1$ is enhanced at higher energies, the contribution of this di: ram becomes less significant as $\sqrt{8} \gg 2 M W$. There is therefore less motivation for excluding these angular regions to hopefully improve the limits on the $\kappa$ couplings. Also, we have found that the other channels, $\mu^{+} e^{-}, \mu^{+} \mu^{-}$, and $e^{+} e^{-}$, because of the extra complexity due to the additional diagrams, both $\kappa v$ dependent and independent, do not as cleanly provide the possibility of sensitivity enhancing cuts. The additional $\kappa_{V}$ dependent diagrams of Figures (3.3) and (3.5) can contribute to the very regions of phase space that we previously considered excluding. For instance, we saw that Figure (3.3a), driven by the phoion propagator, contributes to the angular regions $\cos \theta_{l \pm}= \pm 1$ for both the $\mu^{+} e^{-}$and $e^{+} e^{-}$channels; a cut to exclude these regions of phase space can therefore be counter productive for these processes. 
We have seen that by suitably restricting the full phase space, we can optimize the sensitivity to the vertex couplings, and in some cases significantly improve the limits on these parameters. In general, there will be an optimum cut, maximizing both the, sometimes opposing.requirements of physical sensitivity and total cross-section. This cut is not always immediately apparent; a seemingly over-restrictive cut, thus decreasing cross-section and statistics, call still result in improved limits if the physical sensitivity is increased sufficiently.

\subsection{Discriminating Between $\gamma \mathrm{WW}$ and $\mathrm{ZWW}$}

For each of the four lepton production processes we consider, for every contributing diagram that involves the $\gamma \mathbf{W W}$ vertex, there is a partner, identical but for interchange of $Z$ for $\gamma$. This oneto-one correspondence between $\gamma$ and $\mathrm{Z}$ contributions makes difficult the task of independently constraining the couplings $\kappa_{\gamma}$ and $\kappa_{z}$. We have seen that measurements of total crows-sertion alone, whilst sensitive to variations of $\kappa_{\gamma}$ and $\kappa_{Z}$ away from their SM values, are unable to distinguish between the effects of varying one or the other, or both. This is clear from the contour plots of Chapter 4, Figures (4.6) and (4.7). We saw that the parabolic shape of the full three dimensional surface is such that a horizontal slice, equivalent to a specific non-standard crosssection measurement, determines a contour, or locus of non-standard $\left(\kappa_{\gamma}, \kappa_{z}\right)$ pairs. Any onf choice of $a\left(\kappa_{\gamma}, \kappa_{2}\right)$ pair from this infinite set would give the same total cross-section. We notick also that there are possible pairings that reproduce the suandard model cross-section predictions, thus mimicking the SM pairing of $(1,1)$. Thus, while simple cross-section measurements can , within the limits given previously, determine deviations from the SM, they are unable to pinpoint the values of $\kappa_{\gamma}$ and $\kappa_{z}$ individually.

Various approaches have been proposed that would offer independent information on the two triple boson vertices. One is to investigate processes which involve only one or the other of the two vertices. The associated production of a $W$ with either a gamma or a $Z[39,40,41,42,43]$, radiative $W$ decay $[44,43]$, and ey processes, $[48,49,45,50]$ are some of the poussibilitien. For processes such as we examined, where both of the vertices contribute, the possibility of infplp. menting experimental cuts to isolate one of the two vertices has been examined. For instanir. Godfrey, Couture, and Lewis, [33] examined the $\mu^{+} \mu^{-}$production process as a means of isolating the effects of the $Z W W$ vertice. By requiring the invariant mass of the final $\mu^{+} \mu^{-}$pair to lir within $5 \mathrm{GeV}$ of the $\mathrm{Z}$ mass, the photon contribution is largely excluded, and any evidence of new physics can be attributed to the $\mathrm{ZWW}$ vertex. This idea of making an experimental cut to 
distinguish between the two vertices might also be used to provide limits on $\kappa_{\gamma}$, independent of $\kappa_{Z}$. We will see that by suitable cuts on the angular variable $\cos \theta_{e}-$ in the $\mu^{+} e^{-}$channel, the contributions of the $\mathrm{ZWW}$ vertex can be mimimized, and any limits determined for $\kappa_{\gamma}$ will be independent of $\kappa_{Z}$.

We investigated both these ideas as possible means of independently constraining $\kappa_{\gamma}$ and $\kappa_{Z}$. The results of these studies are presented in the following two Sections. Also, we will see that helicity considerations might provide an additional method of disentangling the effects of the two vertices; we discuss this possibility in Chapter 5.

\subsubsection{Independent $\kappa_{Z}$ Limits}

In this section, we investigate the prospect of placing independent constraints on $\kappa_{\boldsymbol{Z}}$ through the implementation of invariant mass cuts. The $\mu^{+} \mu^{-}$process (as does the $e^{+} e^{-}$process) includes the diagrams of Figure (3.5), with two t-channel W's fusing to a $\gamma(Z)$ decaying to the $\mu^{+} \mu^{-}$pair. These diagrams will be driven by their $\gamma(Z)$ propagators to contribute to different regions of the invariant mass $M_{\mu+\mu^{-}}$phase space. The $\mu^{+} \mu^{-}$and $e^{+} e^{-}$processes thereby afford the prospect of separating the effects of the $\gamma$ and $Z$ contributions.

With no cuts at al! on the $\mu^{+} \mu^{-}$pair invariant mass, the cross-section for the $e^{+} e^{-} \rightarrow \mu^{+} \mu^{-}-\bar{\nu}$ process is dominated by low invariant mass events arising from diagrams involving the photon propagator. This pole appears in a number of Feynman diagrams, both those containing the $\gamma \mathrm{WW}$ vertex and those of photon bremsstrahlung. As was previously mentioned, to regulate these bremsstrahlung events, we imposed a cut requiring that the invariant mass of the $\mu^{+} \mu^{-}$ pair exceed $25 \mathrm{GeV}$. We therefore lose some of the photon contribution and consequently, some sensitivity to the $r W W$ vertex, as the cost of controlling the bremsstrahlung pole. We can even more completely cut out the photon contribution by specifically isolating the $\mathbf{Z}$ diagrams. Referring to Figure (4.13), the differential cross-section as a function of the invariant mass $M_{\mu \mu}$ at $\sqrt{s}=500 \mathrm{GeV}$, we notice that the distribution is dominated by the strong peak at the $Z$ pole. If we restricted phase space to this peak, then we would effectively prevent the photon diagrams from contributing, and thereby isolate the contribution of the $\mathrm{ZWW}$ vertex.

However, in isolating the $Z$ contribution by cutting on the $Z$ peak, we lose the sensitivity to $\kappa_{Z}$ provided by the regions of phase space at higher invariant masses. Figure $(4.13)$ illustrates how the $Z$ peak shows little sensitivity to non-standard $\kappa_{Z}$, it is the plateau region beyond the peak that exhibits the sensitivity. In addition, a cut stringent enough to exclude the photon con- 
tributions will significantly decrease the total cross-section, with consequemly poorer statisticy. With decreased physical sensitivity to $\mathrm{k}_{2}$ and diminished statistics, we should expect. a lows bound on $\mathrm{i}_{\mathrm{Z}}$ than that obtained fron: the full phase space. The advantage is, of course, that the limits placed on $\kappa_{z}$ through studying the restricted phase space are essentially imlependent of variations in $\kappa_{\gamma}$. Thus, any signal of non-standard physics can be attributed to non-standard $\kappa_{Z}$ alone, and not some combination of $\kappa_{\gamma}$ and $\kappa_{Z}$.

To isolate the $Z$ peak, we cut on the invariant mass of the $\mu^{+} \mu^{-}$pair, requiring that it rallge within $\pm 5 \mathrm{GeV}$ of $M_{Z}$. This cut roughly corresponds to $M_{Z} \pm 2 \Gamma_{Z}, \Gamma_{Z}$ being the $Z$ wilth. With this cut in place, we determined the total cross-section for the following three crases of $n$ variation; $\kappa_{\gamma}=\kappa_{Z}, \kappa_{\gamma}=1.0$, and $\kappa_{z}=1.0$. The analysis was performed at the conter-of-Inthss energy of $500 \mathrm{GeV}$. Although we expect no sensitivity for the case of $\kappa_{z}=1.0$, we included it to verify that expected behaviour, ie. relative lack of dependence. Also, to arcount for the experited looser bounds on $\kappa_{Z}$, we allow for $30 \%$ deviation, therefore $\kappa_{v}$ ranges from $0.7 \rightarrow 1.3$. We show in Figure (4.16) the sensitivity ratios, non-standard cross-sections over $\sigma_{S M}$, for the three casses described above. For reference, the standard model cross-section is $\sigma_{S M}=0.0093 \mathrm{pb}$.

We see that the $\kappa_{Z}=1$ case does indeed exhibit the expected insensitivity to variations in $i_{y}$. as exemplified by the relative flatness of its corresponding ratio parabola. "The fact that there is some sensitivity to $k_{\gamma}$ is evidence that the $Z$ peak is not completely free of $\gamma$ contributions.

We obtain limits on $\Delta \kappa_{z}$ as we did in Chapter 4. The equations of the the three parabolats of Figure (4.16) allow us to fit the full 3-D surface plot for total cross-section as a function of $\kappa_{\gamma}$ and $\kappa_{z}$. We show in Figure (4.17) the $2 \sigma$ contour, determined with the assumption of an integrated luminosity of $50 \mathrm{fb}^{-1}$.

The relative insensitivity to $\kappa_{\gamma}$ is apparent from the asymmetric contour shape. The limit.s on $\Delta \kappa_{Z}$ can be read from Figure (4.17); they are approximately

$$
0.82<\kappa_{Z}<1.4
$$

If we compare these bounds to those of Table 4.1 , where the full invariant mass phase spast: was integrated over, we see that, by implementing the cut, we significantly lousen the constraints on variations $\Delta \kappa_{Z}$. The cut $M_{Z} \pm 5 \mathrm{GeV}$ severely reduces both total cross-section and spensitivity to $\mathrm{kZ}_{Z}$, the determining factors for the bounds we obtain. In the hope of tighiening the given limits on $\kappa_{Z}$, we might be tempted to experiment with loosening the cut. However, as we saw, there is, even with the severe $M_{Z} \pm 5$ cut, a slight dependence on the photon diagrams still 


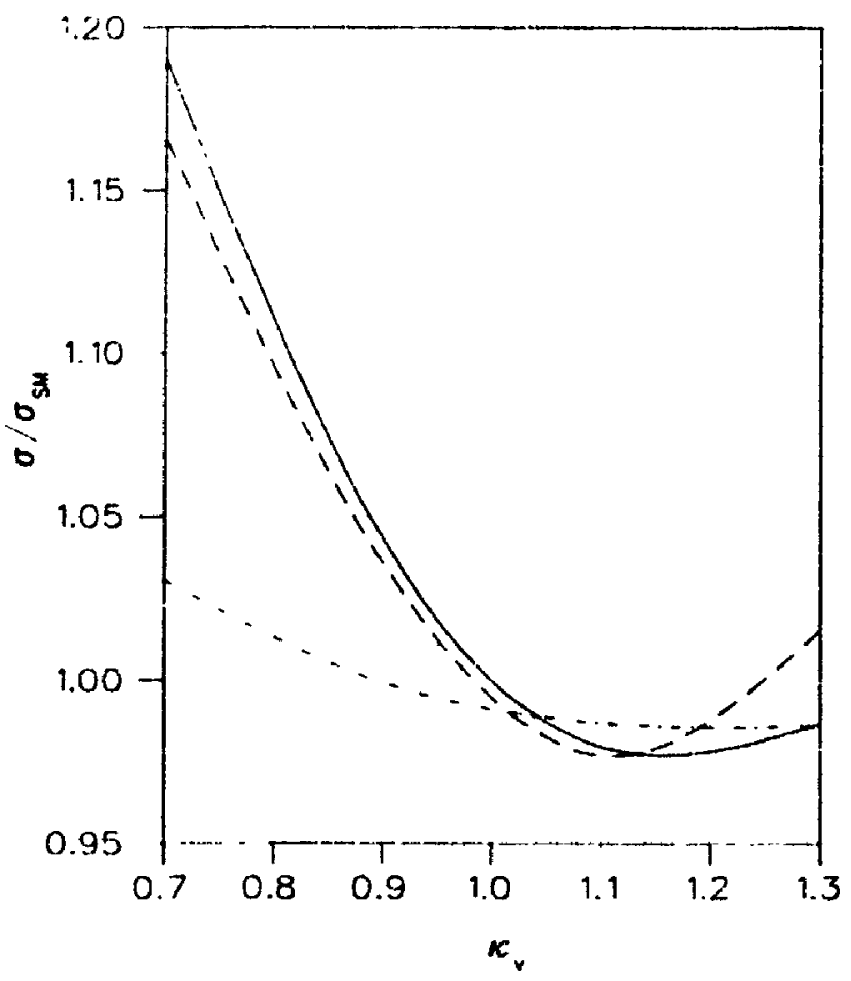

Figure 4.16: Sensitivity Ratios for $\mu^{+} \mu^{-}$process at $\sqrt{5}=500 \mathrm{GeV}$ when $M_{\mu \mu}$ restricted to $M_{Z} \pm 5$ GeV. Solid line corresponds to $\kappa_{\gamma}=\kappa_{Z}$, dashed line to $\kappa_{\gamma}=1.0$, and the dot-dashed line to $\kappa_{Z}=1.0$

contributing to the $Z$ peak region. Loosening the cut would therefore include more of these $\gamma$ contributions, and defeat the original purpose of the cut. It seems, therefore, that the previous limits in $\Delta \kappa_{z}$ could be improved only at the cost of ineffectively excluding the contributions from the $\gamma W W$ vertex.

The final state $e^{+} e^{-}$process also includes the diagrams of Figure (3.5), and so provides the same opportunity for cutting on the $\mathrm{Z}$ peak to isolate the $\mathrm{ZWW}$ vertex. However, not only does $e^{+} e^{-}$have the diagrams of Figures (3.1) and (3.5), (as $\mu^{+} \mu^{-}$does), it also includes those of Figure (3.3). The $e^{+} e^{-}$process is therefore even more likely to have "remnant" photon dependence in the $Z$ peak. We have found that the $e^{+} e^{-}$process is unable to provide independent bounds on $\Delta \kappa_{z}$ that are an improvement on those obtained through the $\mu^{+} \mu^{-}$channel. 


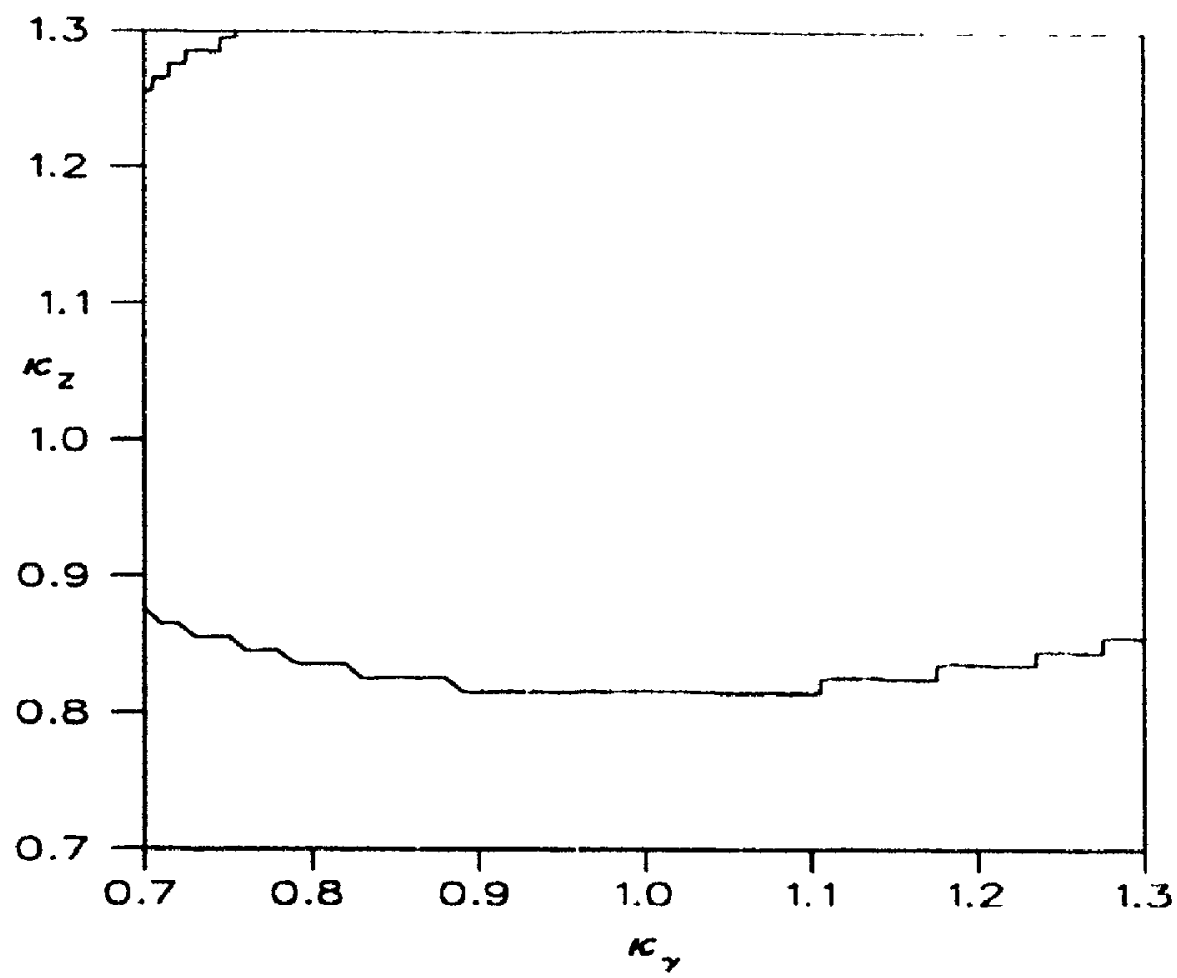

Figure 4.17: $2 \sigma$ contour for $\mu^{+} \mu^{-}$process at $\sqrt{s}=500 \mathrm{GeV}$ when $M_{\mu \mu}$ restricted to $M_{Z} \pm 5$ (ir V.

\subsubsection{Independent $\kappa_{\gamma}$ Limits}

The photon propagator drives the diagram of Figure $(3.3 a)(\gamma)$ to contribute to the angular peak at $\cos \theta_{e}-\sim-1$. If, instead of excluding this peak region as a means of improving overall sensitivity to anomalous couplings, we specifically isolate it, then we minimize the contribution of Figure (3.3a)(Z), and isolate that of Figure $(3.3 \mathrm{a})(\gamma)$. Thus, any of the channels that include Figure (3.3a), $\mu^{+} e^{-}$being the simplest, offer the prospect of offering independent limits on $\kappa_{\gamma}$. As occurred for the case of isolating the ZWW vertex in the $\mu^{+} \mu^{-}$process, we expect lowsu: bounds on $\kappa_{\gamma}$ than those obtained from the full phase space. Our choice of a $\gamma W W$ iscolating cut. must be a compromise between the two opposing requirements of minimizing the ZWW vertex contribution and maximizing the total cross-section. The fact that now we isolate the doninant contribution to the total crosz-section means that we can make a comparatively ighter cul and not lose a prohibitive amount of total cross-section.

We investigated these ideas by examiniag the $\mu^{+} e^{-}$process at a center-of-mast energy of 500 
GeV. We restricted the phase space of the angle $\theta_{e-}$ about the peak such that $18^{\circ}<\theta_{e-}<36^{\circ}$. The left hand limit was motivated by probable detector limitations along the beam line, the right hand limit was arbitrarily chosen as doubling the previuus angle. Recalling that the direction of the incoming positron was defined as the positive z-axis, the above limits correspond to $-0.95<$ $\cos \theta_{e}-<-0.8$. With this cut in place, we determined total cross-sections, and show in Figure (4.18) the cross-section parabolas for the three cases of $\kappa_{\gamma}=\kappa_{Z}, \kappa_{\gamma}=1$, and $\kappa_{Z}=1$.

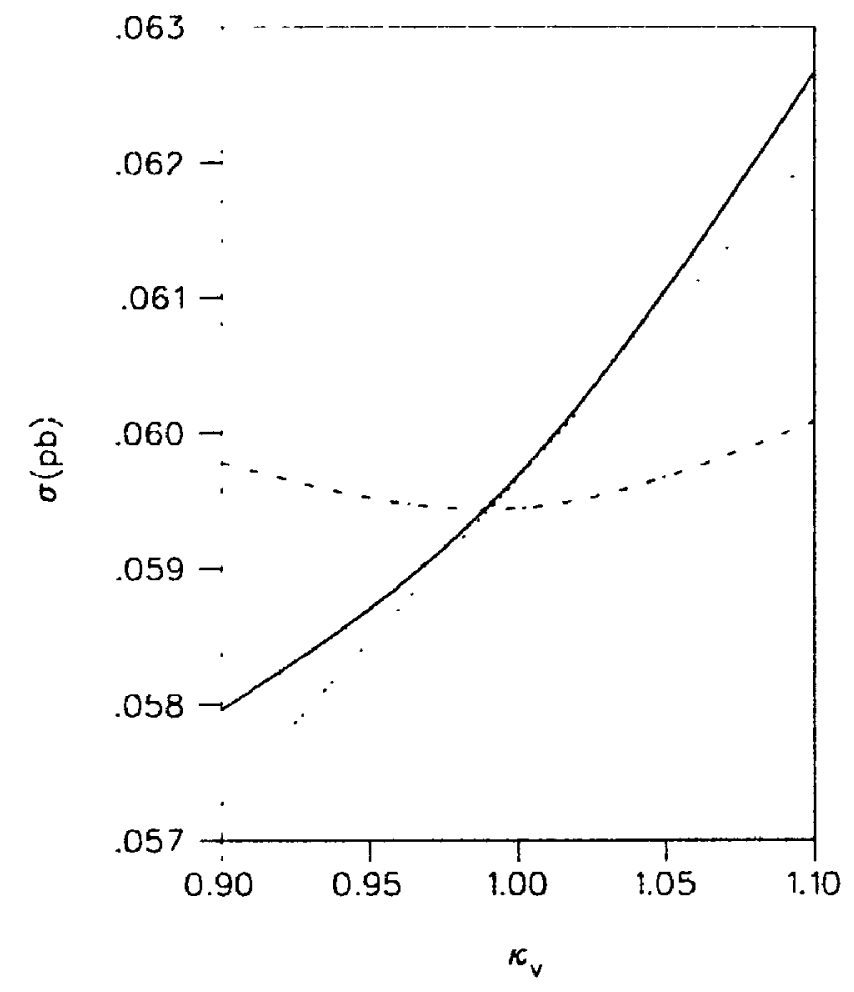

Figure 4.18: Cross-sections for $\mu^{+} \varepsilon^{-}$final state at $\sqrt{s}=500 \mathrm{GeV}$ with $\gamma \mathrm{WW}$ isolating cut $-0.9<\cos \theta_{e}-<-0.8$ implemented. The solid line corresponds to $\kappa_{\gamma}=\kappa_{Z}$, the dot-dashed line to $\kappa_{\gamma}=1$, and the dotted line to $\kappa_{Z}=1$.

As expected, the $\kappa_{\gamma}=1$ curve is relatively flat, reflecting the lack of sensitivity to variations in $\kappa_{z}$. The shapes of the other two curves are very different than those obtained by integrating over the full phase space, $-0.95<\cos \theta_{e-}<0.95$, see Figure (4.2b) These differences reflect how the sensitivities, whether to $\Delta \kappa_{\gamma}$ or $\Delta \kappa_{z}$, are not uniform over the entire range of angular phase space. Thus the ratio curves of the peak region indicate greatest sensitivity to $\Delta \kappa_{\gamma}>0$, those of the full phase space show greatest sensitivity to $\Delta \kappa_{z}<0$. Of course, this variability in "type" 
and degree of sensitivity reflects how the diagrams containing the $7 \mathrm{WW}$ and $2 \mathrm{ZW}$ contribute. in general, to different regions of phase space.

From curves fit to the three sensitivity parabolas of Figure $(4.18)$, the full $3 \mathrm{D}$ cross-section surface was determined. We show in Figure (4.19) the $\pm 2 \sigma$ contours on this 3-D surface.

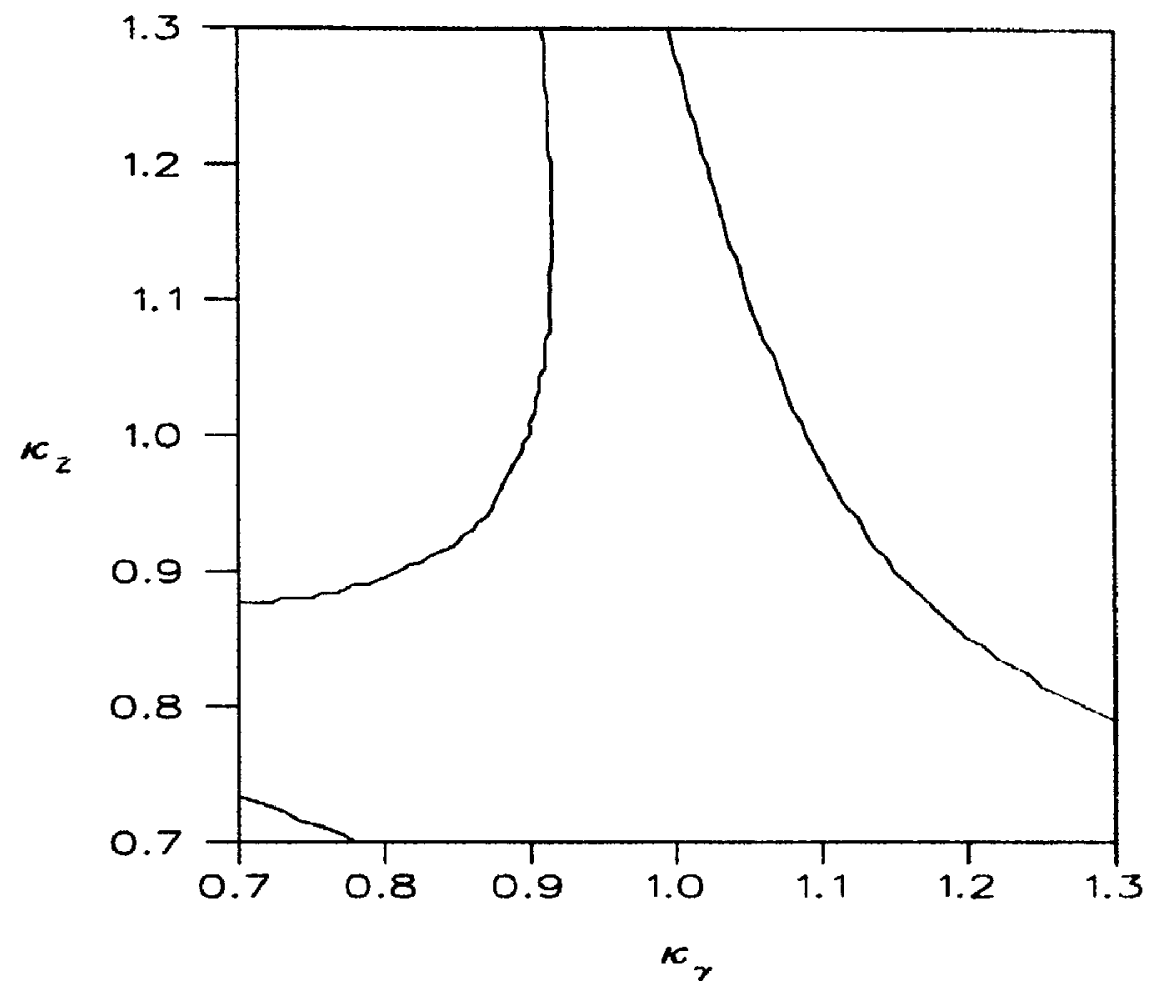

Figure 4.19: $\pm 2 \sigma$ contours in $\kappa_{\gamma}$ and $\kappa_{Z}$ for the case of the $\mu^{+} e^{-}$final state at $\sqrt{s}=500$ ( $\mathrm{reV}$, with the $e^{-}$angular phase space restricted such that $-0.9<\cos \theta_{e^{-}}<-0.8$. Pairings within $Y$-shaped region are indistinguishable from SM.

The difference between these contours and those obtained from integrating over the full phast: space, Figure (4.6b), is immediately apparent. The full phase space 3D surface, the cup of Figure (4.4b), when restricted to the "backwards" region of the $e^{-}$phase space, produces a saddleshaped 3-D surface. We extract independent limits on $\Delta \kappa_{\gamma}$, with $\kappa_{Z}$ restricted to is SM value, from these curves of

$$
-0.1<\Delta \kappa_{-,}<+0.1
$$

These independent $\kappa_{\gamma}$ limits are comparable to those of Couture and Godfrey [63]. They 
exanuined the process $e^{+} e^{-} \rightarrow \gamma \nu_{1} \bar{\nu}_{1}$ for its potential to constrain the WW $\gamma$ vertex independently and predicted bounds at $\sqrt{s}=500 \mathrm{GeV}$ of $-0.05<\Delta \kappa_{\gamma}<+0.05$ at the 95\%C.L. Abraham et al. [64] examined the same process as did Couture and Godfrey but obtain much weaker bounds due to less optimistic assumptions about the size of theoretical systematic errors. McKellar and He [56], in their analysis of recent CLEO data [57] on $b \rightarrow s \gamma$, obtain bounds on $\kappa_{\gamma}$ of $-1.5<\kappa_{\gamma}<1.44$. These bounds are a significant improvement on those predicted from analysis of associated $W$ production at Tevatron energies [43] and are comparable to limits predicted from the same process but at LHC energies [40]. They are comparable to the predicted limits from an $e \gamma$ collider at $\sqrt{s}=120 \mathrm{GeV}$ [48], but significantly weaker than those for a $1 \mathrm{TeV}$ eq collider [49].

We have seen that by making suitable cuts on the phase space of certain experimental observables, it might be possible to obtain independent limits on the couplings $\kappa_{\gamma}$ and $\kappa_{Z}$. The achievable limits, although weaker than those obtainable from the full cross-section, allow us to disentangle the effects of the two vertices. 


\section{Chapter 5}

\section{Initial Beam Polarization}

In the energy ranges of LEP and higher, in contrast with the left-right symmetric physics of the QED dominated lower energies, helicity effects are expected to be important. These polarization effects might prove useful in the measurement of the triple boson vertices $W W \gamma$ and $W W Z$. 'The possibility of using polarized $e^{+} e^{-}$beams to access the helicity information in a measurement of the TBV has been examined previously. Blondel et al. [65], in their analysis of the $e^{+{ }^{-}-} \rightarrow$ $W^{+} W^{-}$process at LEP2 energies, argued that polarized beams would not provide a substantial gain in sensitivity, but could help to disentangle different anomalous contributions. Perkins [66], in his review of the physics potential of LEP2, reaches the same conciusion; if measurable deviations from the SM are observed with unpolarized beams, polarized beams could be used to investigate the anomaly. Bilchak and Stroughair [30], in their study of $\mathrm{W}$ pair production at a $\sqrt{s}=260 \mathrm{GeV}$ polarized $e^{+} e^{-}$collider, conclude that much better limits could be achieved than at LEP200. Likhoded et al. [38], in examining the same process at the higher CM energies of $0.5-1 \mathrm{TeV}$, found that significantly improved limits on the couplings could be achieved with initial beam polarization. Philipsen [67] examined the $e \gamma \rightarrow W \nu$ process at a polarized et ${ }^{+}-$ collider and found that the cross-section was no more sensitive to $\kappa_{\gamma}$ for polarized beams than for unpolarized beams. Anomalous values for $\lambda_{\gamma}$ do however discriminate between opposite polarization states; polarization might therefore prove useful for such anomalous couplings.

In this Chapter, we investigate the potential for improving the previously determined detec:tion limits on $\kappa_{\gamma}$ and $\kappa_{z}$ of Chapter 4 through accessing the information contained in the helicity structure. The fact that the Neutral current couplings are different for left and right-handed fermions, and that the Feynman diagrams containing the triple boson vertex do not contributs: 
to all helicity amplitudes, ensures that the different helicity amplitudes can have very different dependence on $\kappa_{-}$and $\kappa_{Z}$, relative to each other and the total cross section. The contributions of these different helicity amplitudes to the total cross section can therefore provide constraints on $\kappa_{\gamma}$ and $\kappa_{z}$ that are complementary to those obtained from the total cross section. We will see that the ability to separately measure the contributing helicity amplitudes to a given process will improve both the overall detection limits for non-standard $\kappa_{\gamma}$ and $\kappa_{z}$ as well as our ability to pinpoint the origin of a non-standard cross section measurement.

In a realistic experiment, longitudinal polarization will not be perfect. In practice, we might achieve average degrees of polarization for the electron and positron characterized by $P_{1}$ and $P_{2}$ respectively. Thus, a "right-handed" positron and "left-handed" electron would be described by $P_{1}=-0.8$ and $P_{2}=0.8$, instead of the ideal -1 and 1 . The significance of these average polarizations is that the experimentally measured component helicity cross sections will also contain a contribution from the other helicity states. For instance, the two helicity amplitudes that contribute to the $\mu^{+} \tau^{-}$channel, $(+-+-)$and $(-++-)$, will not be perfectly resolvable experimentally. In practice then, the cross section will be

$$
\sigma=\frac{1}{4}\left[\left(1+P_{-}\right) \cdot\left(1-P_{+}\right) \sigma^{-+}+\left(1-P_{-}\right) \cdot\left(1+P_{+}\right) \sigma^{+-}\right]
$$

In addition to the limitations on polarization which prevent unambiguous identification of a specific helicity cross section, there will be uncertainty on the actual polarizations achieved, further confusing the issue. However, as we are more interested in determining the scale of the possible improved limits for $\kappa_{V}$. than deriving rigorous values for them, we neglect in the following these polarization limitations and uncertainties. We therefore assume $100 \%$ longitudinal polarization for the initial electron and positron, with zero uncertainty. We investigate in the following Sections the advantages offered by a polarized beam facility, both in improving the overall bounds on anomalous $\kappa_{V}$, Section 5.1, as well as distinguishing $\kappa_{\gamma}$ and $\kappa_{z}$ effects, Section 5.2.

\subsection{Improving $\kappa_{V}$ bounds Through Polarization Studies}

Thus far, the cross sections we have examined, both integrated and differential, have included all the component helicity amplitudes. A significant improvement on these limits might be possible if the information contained in the individual helicity amplitudes could be accessed, ie. if initial 
$e^{t} e^{-}$longitudinal polarization was available. With such initial state polarization. We could separately measure the cross sections for the contributing helicity amplitudes.

The individual amplitudes can differ in their dependence, both in form and magnitude, on the triple boson vertices; thus by separately measuring the component helicity cross swetions. we can optimize the overall sensitivity to $\mathrm{kV}$. For instance, the $\mu^{+} \tau^{-}$process has the two component amplitudes $(+-+-)$ and $(-++-)$, the first involves the neutrino exchange diagram of Figure (3.4a), the second does not. ' This diagram dominates the $(+-+-)$ amplitude is well as the total cross section but, unfortunately, does not contain the trilinear couplings. 'The neutrino exchange diagram can therefore be thought of as a $\mathrm{n}_{\mathrm{V}}$-independent "background" 1.0 the contributions of the triple boson vertex containing diagrams. The second amplitude, and resulting cross section, is free of the large $\kappa_{V}$-independent contribution of this diagram. and therefore has a relatively greater sensitivity to deviations $\Delta \kappa v$. Separately masusuing the two helicity contributions would effectively cut out the $\kappa$-independent background, with consequently improved sensitivity. Without the dominant t-channel neutrino contribution however, the crows section $\sigma_{-++-}$is much smaller than that of $\sigma_{+-+-}\left(\sigma_{-++-} \sim 10^{-2} \sigma_{+-+-}\right)$, with consequently poorer statistics. It therefore remains to be seen whether the improved sensitivity to $\Delta$ nit $^{\prime}$ of $\sigma_{-++-}$will be sufficient to counter the loss of statistics, and ultimately provide more stringent. limits.

To illustrate the scale of the possible improvements in the detection limits for the deviations $\Delta \kappa v$, we examine the individual helicity amplitude contributions to the total cross sections for the $\mu^{+} \tau^{-}$and $e^{+} e^{-}$channels at $\sqrt{s}=500 \mathrm{GeV}$. The $\mu^{+} \tau^{-}$channel is chosen because, with only two helicity amplitudes contributing, it provides the simplest demonstration of the ideass discussed; the $e^{t} e^{-}$channel, with all six possible helicity amplitudes present, demonstrates hirw the necessary summation over amplitudes can "blur" the well-defined individual amplitude limits. Without the ability to measure the polarization of the final state fermions, what would actually be measured are the four different possible combinations of initial polarizations,,,+++--+ . and -- , some of which would be sums over separate helicity amplitudes. Therefore, in the $t_{, \ldots}-$ channel, the amplitudes $(+-+-)$ and $(+--+)$ would be experimentally indistinguishable, thus they would both contribute to the combined cross section +- .

As before, we explore the sensitivity of the cross sections to variations in $\kappa_{\gamma}$ and $\kappa_{z_{1}}$, Int now also use our Monte Carlo program to determine the percentage contribution of each helicity

\footnotetext{
${ }^{1}$ We denote the helicities of the incoming positron, incoming electron, outgoing charged antilepton, and istgoing charged lepton as $(\bar{\alpha} \beta \bar{\rho} \psi)$, respectively.
} 
state to the total cross section. We then convert these percentages into effective component cross sections, from which helicity detection limits on $\kappa_{\gamma}$ and $\kappa_{z}$ can be determined. The Standard Model total cross section for the $\mu^{+} \tau^{-}$process at $\sqrt{s}=500 \mathrm{GeV}$, accounting for the charge conjugate channel $\mu^{-} \tau^{+}$, is $\sigma_{S M}=0.0684 \mathrm{pb}$. The individual helicity state contributions to this total are determined to be, $\sigma_{S M}^{(+-)^{+-}}=0.0676 \mathrm{pb}$ and $\sigma_{S M}^{(-++-)}=0.0008 \mathrm{pb}$. As for the total cross section, we fit the component cross sections $\sigma_{(+-+-)}$and $\sigma_{(-++-)}$as multivariable functions of $\kappa_{\gamma}$ and $\kappa_{Z}$, thus determining the equation of the 3-D surfaces for the component cross sections.

We show in Figure (5.1) the full 3-D lego plots for the cross sections $\sigma_{(+-+-)}$and $\sigma_{(-++-)}$.

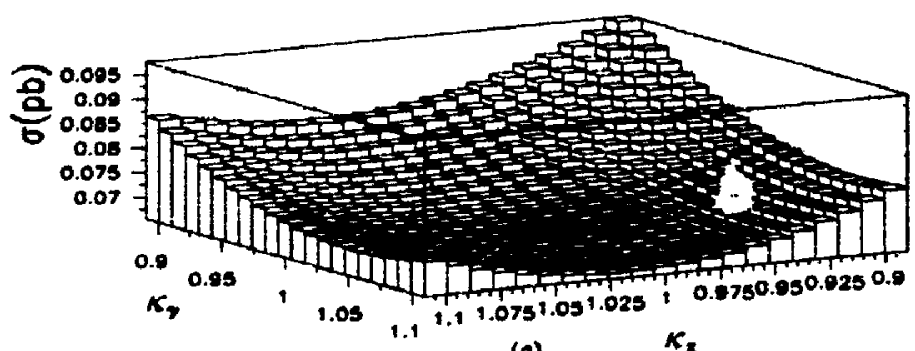

(a)

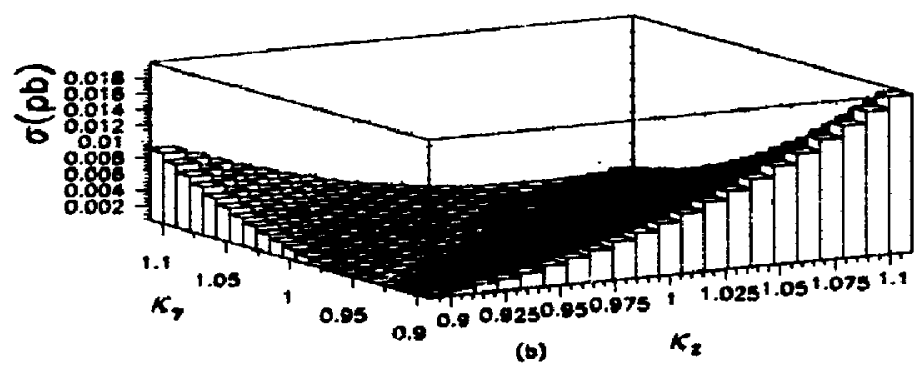

Figure 5.1: Cross-section as function of $\kappa_{\gamma}$ and $\kappa_{Z}$ for $(a)(+-+-)$ helicity amplitude and (b) $(-++-)$ helicity amplitudes of $\mu^{+} \tau^{-}$channel at $\sqrt{s}=500 \mathrm{GeV}$. The plot for the $(-++-)$ amplitude has been rotated $180 \mathrm{deg}$ for ease of viewing.

The shape of the surface for $\sigma_{(+-+-)}$, Figure (5.1a), is very sirnilar to that of the total crosssection, see Figure (4.4a). This is not surprising; the helicity state $(+-+-)$ dominates the total cross section and so should also govern the form of the dependence on $\kappa \mathrm{V}$. The surface for the $\sigma_{(-++-)}$cross-section, Figure (5.1b), appears very different from either that of $\sigma_{(+-+-)}$or the total cross-section. Instead of a parabolic shape about a unique mimimum, $\sigma_{(-++-)}$exhibits a "valley-like" shape about the minimum running roughly parallel to the $\kappa_{\gamma}=\kappa_{z}$ diagonal. We 
will see that this form for the surface is a result of the cancellations that oceur between the photon and $Z$ contributions to the $(-++-)$ amplitude for such equal pairings of $k$, and $k \%$.

We perform the statistical analysis to determine the $\sigma_{s}$ a $\pm 2 \sigma$ cross sections abum the Standard Model values, we show these contours in Figure (5.2). The concentric solid lines atre the $\pm 2 \sigma$ contours for the $(+-+-)$ helicity state: the "disk" they define is very similar to that of the unpolarized case, see Figure (4.6a). The four parallel diagonal dotted lines are the $\pm 2 \sigma$ contours corresponding to the $(-++-)$ amplitude. This distinctive form is determined by the shape of the 3-D surface, Figure (5.1b). The SM pairing is located on one "hank" of the valley of the 3-D surface plot, there are however additional pairings on the other "bank" that atr" also, statistically compatible with the SM pairing. The "allowed" regions for the couplings $\kappa_{\gamma}, \kappa_{Z}$, ins constrained by this helicity state, are thus the bands running parallel to the $\kappa_{\uparrow}=\kappa_{2}$ dingonnl.

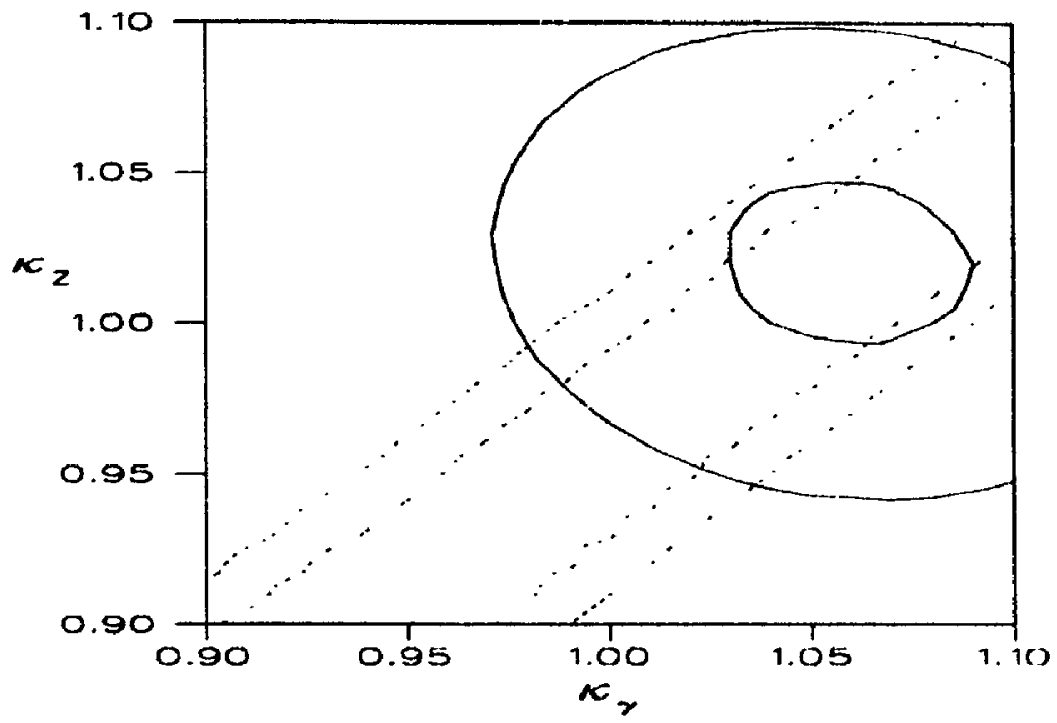

Figure 5.2: $\pm 2 \sigma$ contour plots for dominant $(+-+-)$ helicity amplitude (solid line) and $(-++-)$ helicity amplitude (dashed line) of $\mu^{+} \tau^{-}$channel at $\sqrt{\delta}=500 \mathrm{GeV}$.

Neither the $(+-+-)$ disk region or the $(-++-)$ bands independently offer significantly tighter constraints on $\kappa_{\gamma}$ and $\kappa_{Z}$ than did the unpolarized cross section; the intersection of thr. former with the latter does however severely restrict the allowed domain. What had bren a relatively large set of $\kappa_{\gamma}, \kappa_{Z}$ pairings that were statistically indistinguishable from the Standard Model prediction is reduced to a significanily smaller union of two separate resgions. Polarization measurements could therefore, in the specific case of the $\mu^{+} \tau^{-}$final state, provide signifirantly 
tighter constraints on $\kappa_{\gamma}$ and $\kappa_{z}$ than would the unpolarized cross section. For instance, if we vary the couplings individually, keeping the other at its SM value, we extract limits from the contours of Figure (5.2) at the 95\% C.L. of

$$
\begin{aligned}
& 0.9<\kappa_{Z}<0.93 \text { and } 0.99<\kappa_{Z}<1.01 \\
& 0.98<\kappa_{\gamma}<1.01 \text { and } 1.07<\kappa_{\gamma}<1.09
\end{aligned}
$$

which compare favourably with the bounds from the unpolarized analysis.

We repeated this polarization analysis for the $e^{+} e^{-}$channel at $\sqrt{8}=500 \mathrm{GeV}$. As was previously mentioned, although there are six different helicity amplitudes that contribute to the $e^{+} e^{-}$ channel, without the ability to measure the polarizations of the final state leptons, we have only four experimentally distinguishable helicity states,,,--+--+ and ++ . Thus -+ is the sum of the indistinguishable $(-++-)$ and $(-+-+),+-$ the sum of $(+-+-)$ and $(+--+)$, ++ and -- are composed of the individual amplitudes $(++++)$ and $(----)$ respectively. This summation over helicity states might complicate the effect observed in the simpler $\mu^{+} \tau^{-}$ situation. For instance, the distinctive shape of the $(-++-)$ 3-D surface, Figure (5.1b), in the $\mu^{+} \tau^{-}$case was that which determined the $\kappa_{V}$ constraining region of intersection. For the $e^{+} e^{-}$ process, the comparatively small contribution of the $(-++-)$ state is added to that of $(-+-+)$, its distinctive dependence on $\kappa_{\gamma}$ and $\kappa_{z}$ could thus be "swamped" by the larger contribution of $(-+-+)$.

We find that this is indeed the case. In Figure (5.3) we show, for the $e^{+} e^{-}$channel at $\sqrt{s}=500 \mathrm{GeV}$, the $\pm 2 \sigma$ contours in $\kappa_{\gamma}$ and $\kappa_{z}$ for the different helicity states +- and -+ . Not shown are the contours for the ++ and -- helicity amplitudes, their relative lack of sensitivity to $\mathrm{kV}$ prevented them from placing any significant constraints on the couplings additional to those from the +- and -+ helicity amplitudes. 


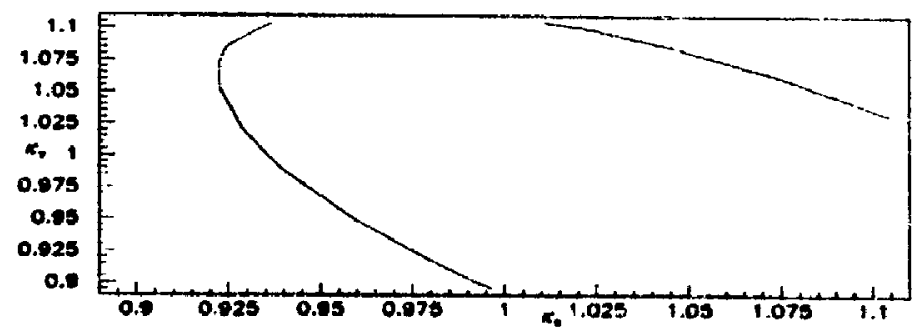

(a)

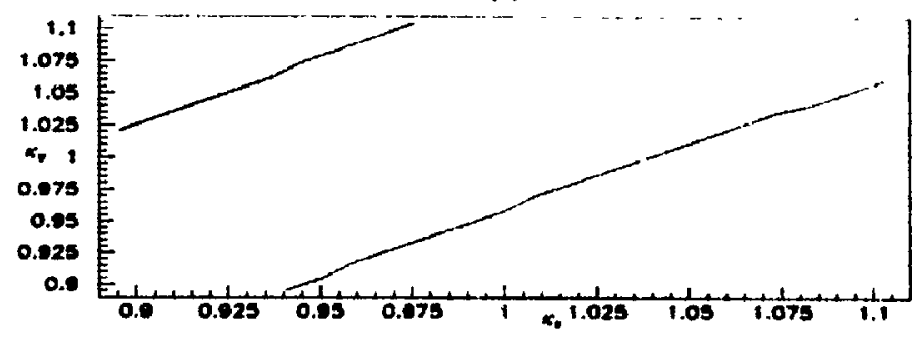

(b)

Figure 5.3: $\pm 2 \sigma$ contour plots in $\kappa_{\gamma}$ and $\kappa_{z}$ for $(a)+-$ and (b) -+ combined helicity amplitudes for $e^{+} e^{-}$channel at $\sqrt{s}=500 \mathrm{GeV}$.

The +- cross section contains the dominant contribution of the $(+-+-)$ amplitude, its corresponding $+2 \sigma$ contour, Figure (5.3a) is similar in shape to that of the total cross section, but less constraining. The $\pm 2 \sigma$ contours for the -+ cross section, Figure(5.3b), did not determine two "parallel" bands as in the $\mu^{+} \tau^{-}$analysis, see Figure (5.2). Thus, although the allowed pairinge of $\kappa_{\gamma}$ and $\kappa_{z}$ are constrained to the intersection of the regions defined by the contours of Figures (5.3a) and (5.3b), limits taken from the boundaries of this intersection region are no improvement on the unpolarized case. The distinctive shape of the $(-++-)$ 3-D surface, as seen in the simpler $\mu^{+} \tau^{-}$case, Fig. (5.1b), is somewhat degraded in the $e^{+} e^{-}$process. Here, the $(-++-)$ contribution must be added to that of $(-+-+)$, this extra piece having very different $\kappa_{V}$-dependence, in degree and form. Thus, the distinctiveness of the $(-++-)$ cross section surface, necessary for the constraining effect seen in the $\mu^{+} \tau^{-}$analysig, is lost; with a consequent lack of improvement in $\kappa_{V}$ limits. 


\subsection{Polarization as means of Differentiating between $\kappa_{\gamma}$ and $\kappa_{Z}$.}

We explored in Section 4.4 the idea of making suitable cuts to isolate the effects of either the $W W_{\gamma}$ or the WWZ vertex and, thereby, determine independent limits on $\kappa_{\gamma}$ and $\kappa_{z}$. The fact that the photon couples equally to both left and right handed fermions, and the $\mathrm{Z}$ boson couples differently to the two helicity states, might suggest an additional method of "untangling" the effects of the two different triple boson vertices. Because of this helicity asymmetry in the $Z$ couplings, cancellations in the component helicity amplitudes occur for certain combinations of $\kappa_{\gamma}$ and $\kappa_{z}$ thus produring a characteristic signal for these special pairings. Because it has only two contributing helicity amplitudes, we use the $\mu^{+} \boldsymbol{r}^{-}$production process to examine this poesibility. In principle, it is valid for the other channels, but, for these others, the additional helicity amplitudes can complicate the effect.

As described above, the $\mu^{+} \tau^{-}$channel has contributions from the $(+-+-)$and $(-++-)$. helicity amplitudes. The $(+-+-)$ amplitude corresponds to an incoming right-handed positron and left-handed electron producing a right and left-handed outgoing $\mu^{+}$and $\boldsymbol{r}^{-}$, respectively. Because all of the nine contributing diagrams have the outgoing fermions linked, either directly or indirectly, to a $\mathbf{W}$ boson, which couples only to left-handed leptons (or right-handed antileptons), the other possibility of left-handed $\mu^{+}$and right-handed $\boldsymbol{r}^{-}$does not occur. Similarly, $(-++-)$ corresponds to having the helicities of the incoming state flipped, thus a left-handed positron and right-handed electron produce the same (as the other helicity amplitude) final state.

The sensitivity to the two triple boson vertices in the $\mu^{+} T^{-}$channel comes from the two diagrams of Figure (3.1); they differ only in the two vertices, eeV and VWW ( $V$ being either $\gamma$ or Z), and the s-channel neutral boson propagator. Although it is the vertex VWW in which we are interested, it is the other vertex, between the incoming fermions and the s-channel boson, $\gamma$ or $\mathrm{Z}$, that could provide the means of further untangling the effects of the two triple boson vertices. The key is in how the $Z$ boson couples differently to left and right-handed fermions.

The Standard Model Feynman rules for the couplings of photons and $\mathrm{Z}$ bosons to massive leptons are respectively,

$$
\begin{gathered}
\Gamma_{\mu}^{\gamma}=-i e \gamma_{\mu}\left(P_{L}+P_{R}\right) \\
\Gamma_{\mu}^{Z}=\frac{-i e}{\sin \theta_{W} \cos \theta_{W}} \gamma_{\mu}\left(\left(-1 / 2+\sin ^{2} \theta_{W}\right) P_{L}+\left(\sin ^{2} \theta_{W}\right) P_{R}\right)
\end{gathered}
$$


where

$$
P_{L}=\frac{1-\gamma_{5}}{2} \quad P_{R}=\frac{1+\gamma_{5}}{2}
$$

These rules, plus the different coupling strengths of the two triple boson vertices $\gamma W W$ and ZWW, ensure that the $\kappa$-dependence of the $(-++-)$ amplitude can be schematically written as

$$
M_{(-++-)}=\left(\frac{\kappa_{y}-1}{s}-\frac{\kappa_{Z}-1}{s-M_{2}^{2}}\right)(A)+(B)
$$

Thus, if $s>M_{Z}^{2}$, as is true for the energies we consider, then for equal values of nonstandard $\kappa_{\gamma}$ and $\kappa_{z}$, the different contributions of the photon and $Z$ bosons will nearly rancel. The $(-++-)$ amplitude contribution will be consequently diminished, relative to pairings where $\kappa_{\gamma}$ and $\kappa_{Z}$ are unequal. This behaviour manifests itself in the shape of the 3-D surface for the dependence of the $\sigma^{-++-}$cross section on $\kappa_{\gamma}$ and $\kappa_{z}$, Figure (5.1b). The dominant $(+-+-)$ amplitude, because the coupling of the $Z$ boson to a left-handed lepton does not have the simple $\sin ^{2} \theta_{W}$ dependence of the right-handed lepton, does not exhibit such cancellation between the photon and $Z$ contributions for equal $\kappa_{\gamma}$ and $\kappa_{Z}$. With the $(-++-)$ anplitude relatively diminished for equal $\kappa_{\gamma}$ and $\kappa_{Z}$, and the $(+-+-)$ amplitude unaffected, such equality betwesu the couplings should manifest itself as a relatively small contribution of $(-++-)$ to the total cross section, compared to the case when $\kappa_{\boldsymbol{\gamma}} \neq \kappa_{z}$.

For large center-of-mass energies, the cancellation between the photon and $\mathrm{Z}$ terms whell $\kappa_{\gamma}=\kappa_{Z}$, either standard or non-standard, results in a $(-++-)$ helicity amplitude contribution of less than about $1 \%$ of the total cross section. When $\kappa_{\gamma} \neq \kappa_{z}$ however, this amplitude can contribute as much as $70 \%$ of the total, the above cancellation being destroyed. A small percentage contribution of $(-++-)$ to the total is therefore characteristic of equal values for $\kappa_{\gamma}$ and $\kappa_{z}$ and, thus, provides a characteristic "signature" of such pairings. Once again, because it offers the simplest example, we examine the $\mu^{+} \tau^{-}$channel in the following discussion.

We show in Figure (5.4) the differential cross section with respect to total visible transverse: momentum for various pairings of $\kappa_{\gamma}$ and $\kappa_{Z}$, determined at $\sqrt{8}=1 \mathrm{TeV}$. For each of the three pairings of $\left(\kappa_{\gamma}, \kappa_{z}\right):(1.0,1.0),(0.9,1.0)$, and $(0.9,0.9)$, the solid line represents the total unpolarized cross section, the dashed line corresponds to the dominant $(+-+-)$ contribution, and the dotted line to the amplitude of interest $(-++-)$. 

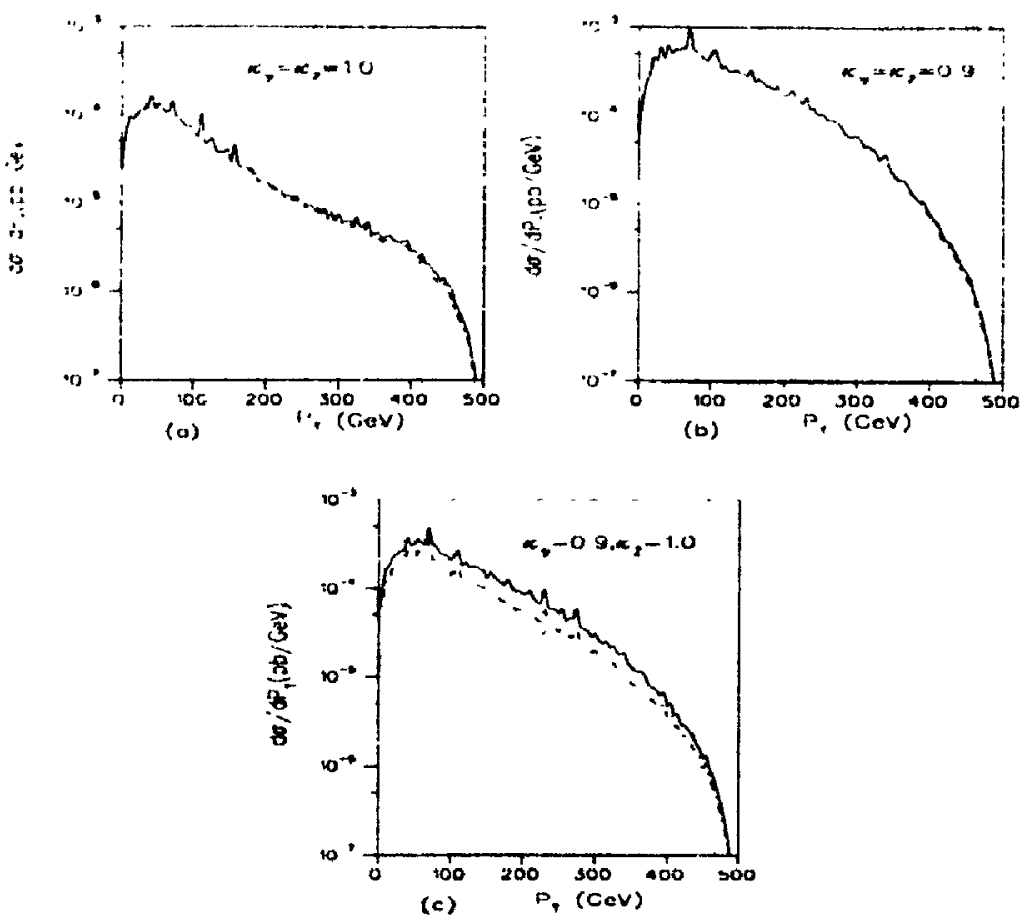

Figure 5.4: Differential cross section with respect to total visible transverse momentum for unpolarized cross section ( solid line ), $(+-+-)$ amplitude (dot-dashed line ), and $(-++-)$ amplitude (dotted line ) for the combinations of $\left(\kappa_{\gamma}\right.$ and $\kappa_{Z}:(1.0,1.0)(a),(0.9,1.0)(b)$, and $(0.9,0.9)(c)$ for the $\mu^{+} \tau^{-}$process at $\sqrt{s}=1 \mathrm{TeV}$.

The distributions, both total and individual helicity amplitudes, look similar for the two rases of equal $\kappa v$, Figures (5.4a) and (5.4b). There is an overall enhancement in $(+-+-1$, and consequently the total differential cross section for the non-standard pairing. The $(-++-)$ contribution appears almost identical in shape and magnitude for these equal $\kappa_{V}$ cases. We can understand this by referring to Eq. (5.4). When $\kappa_{\gamma}=\kappa_{Z}$, the first term, multiplying (A), will be very small; the $(-++-)$ contribution will therefore consist for the most part of the (B) term, with no $\kappa_{V}$ dependence. Therefore, this contribution is mostly unchanged for all equal $\kappa_{V}$ pairings, the sinall differences that do exist are due to imperfect cancellation of the (A) term.

When $\kappa_{\gamma} \neq \kappa_{Z}$, as in the case of Fig. (5.4c), no such cancellation occurs, and the $\kappa_{V}$ dependent (A) contribution of Eq. (5.4) can be significant. In Fig. $(5.4 \mathrm{c})$, the $(+-+-)$ distribution's appearance is not significantly different from the same helicity state for the other toon-standard, but equal, pairing of $\kappa_{\gamma}$ and $\kappa_{Z}$, Fig. (5.4b). The $(-++-)$ contribution is 
however very different; the uncancelled (A) term produces a significant contribution beyond that $\kappa_{1} \cdot$-independent (B) term. With such significant differences in the distributions of the cont. ponent helicity amplitudes for the two possibilities of equal or Honecpanl $\mathrm{si}_{\mathrm{i}}$, the pussibility of differentiating between these cases with polarized heams is suggestect.

More than merely helping to distinguish between the casses of equal or unequal $h$, and $n \%$. the relative contribution of the $(-++-)$ amplitude could potentially serve ats a chatrateristic "fingerprint" of a particular region of the $\kappa_{9}, k_{z}$ grid. To explote this tedea, we determined the percent contribution of the $(-++-)$ amplitude to the total cross section for values of $\mathrm{k}$, and $\kappa_{Z}$ ranging from 0.9 to 1.1. We continued with the $\mu^{+} \tau^{-}$channel but performud the athalysis it a center-of-mass energy of $\sqrt{s}=500$ GeV.

The contributions ranged from a low of $0.75 \%$ for the pairing of $\left(\kappa_{\gamma}=0.9, \alpha_{z}=0,4\right)$, tw it high of $29.3 \%$ for a pairing of $(0.9,1.1)$. As expected, the greatest contribution of the $(-++-1$ amplitude to the total occurs when the difference between $\kappa_{\gamma}$ and $\kappa_{z}$ is maximizarl. $A$ s we diul for the total cross section in Chapter 4. we can deternine the 3-D surface of percentage contribution as a function of $\kappa_{\gamma}$ and $\kappa_{z}$. Specific levels of percent contribution. when plotum on this 3 D surface, will determine contours; thus defining regions of the $\kappa_{\gamma}, \kappa_{Z}$ grid with iharateristive $(-++-)$ contributions. We show these contours in Figure (5.5). 

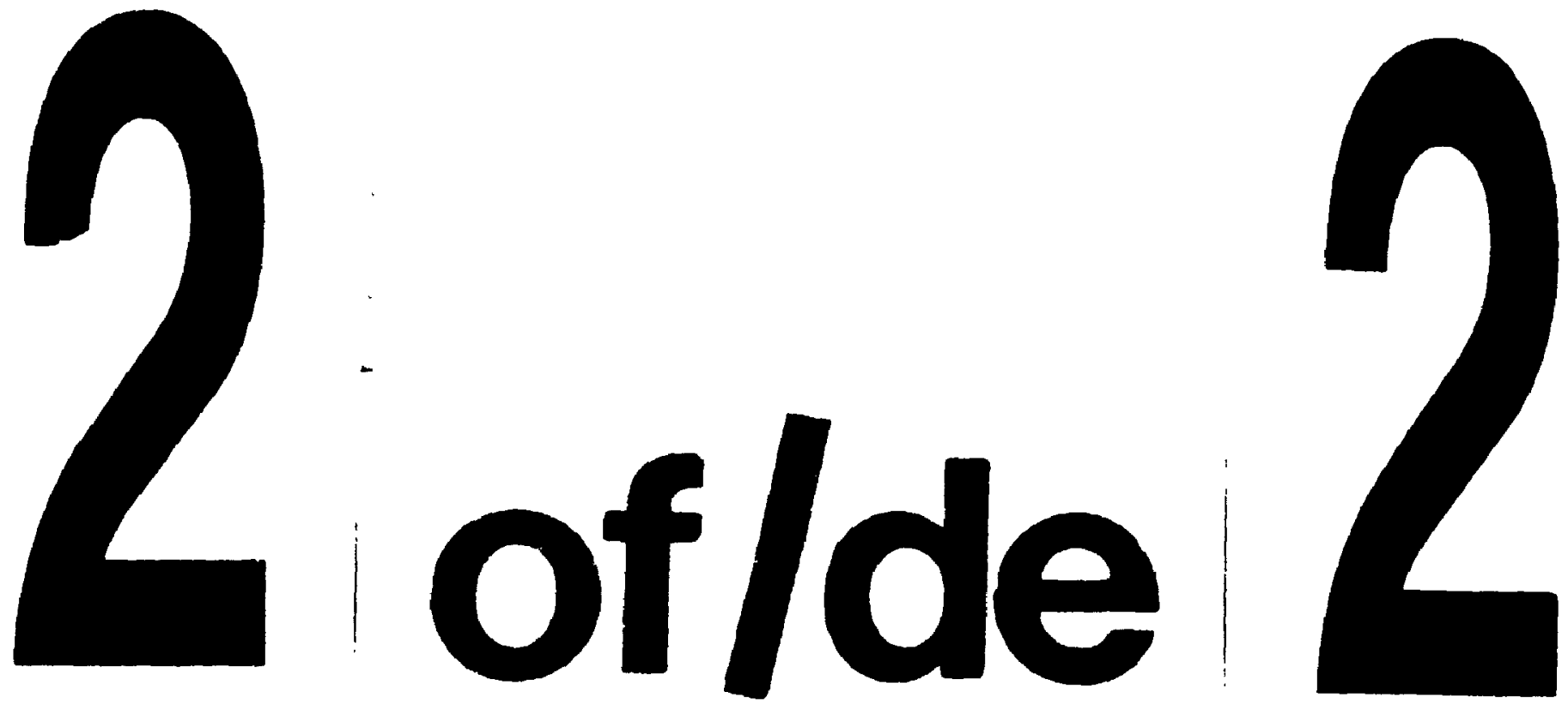

PM-1 31/2"X4" PHOTOGRAPHIC MICROCOPY TARGET NES 1010 a ANSI/ISO \#2 EOUIVALENT

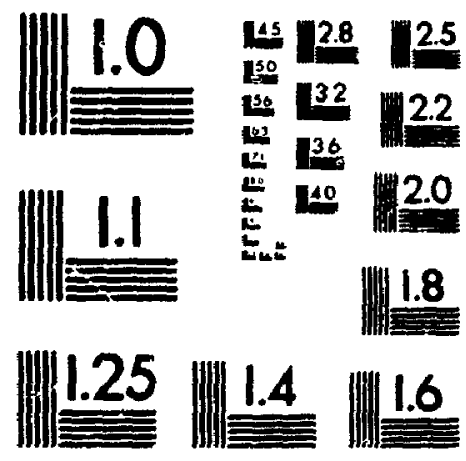

PAECISIONSW RESOLUTION TARGETS 


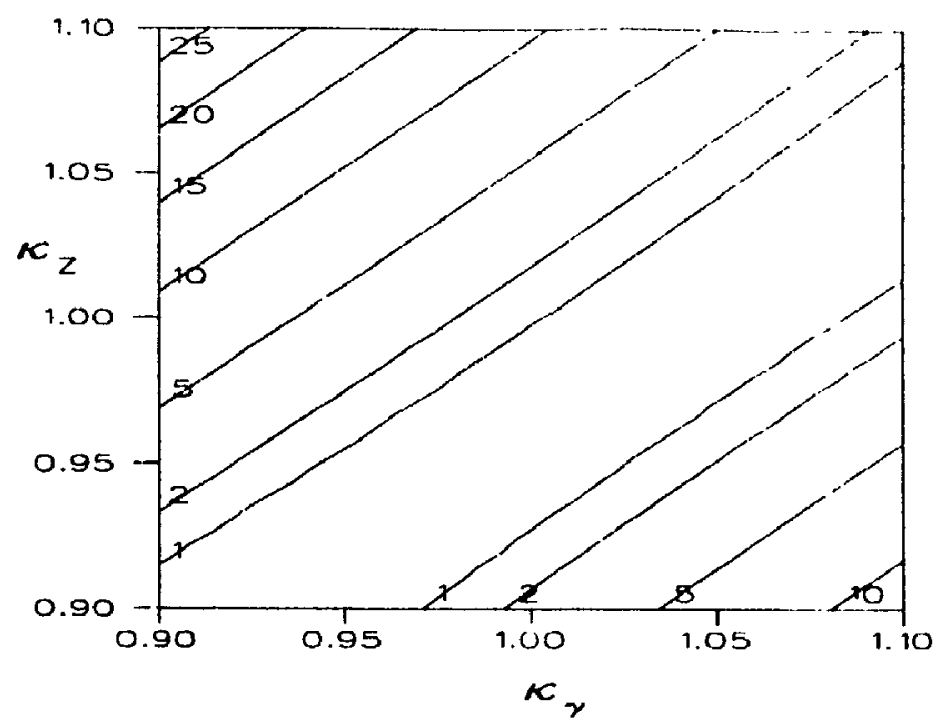

Figure 5.5: Percent contributions of $(-++-)$ helicity amplitude to total cross-section as function . of $\kappa_{\gamma}$ and $\kappa_{Z}$ for the $\mu^{+} \tau^{-}$process at $\sqrt{s}=500 \mathrm{GeV}$.

An experimental measurement of the $(-++-)$ percent contribution of, for example, s) $\mathrm{M}$, would indicate a very different locus of possible non-standard pairings than would, say, $0.5 \%$ or $10 \%$.

The predicted level of $(-++-)$ contribution in the Standard Model, with $\kappa_{\gamma}=\kappa_{Z}=1.0$, is approximately $1.2 \%$. By assuming a Confidence Level of $\pm 2 \sigma$ for the experimental determination of this value, we find, with $\sigma_{S M}=0.034 \mathrm{pb}$ and assuming an integrated luminosity of $50 \mathrm{sb}^{-}$: that the contribution might be measured within the approximate range $0.65 \% \rightarrow 1.4 \%$. 'That is, percent contributions outside this range would be inconsistent, at the given C.I., with thr. Standard Model, and could be interpreted as evidence of non-standard physics. However, the shape of the 3-D surface, Figure (5.1b), is such that the given limits for the percent contribution would define two bands of "allowed" pairings, running parallel to, and on either side of the minimum diagonal. Because these bands would contain a not insignificant portion of the $\kappa_{\gamma}, \kappa_{z}$ grid, an analysis of $(-++-)$ contributions would, on its own, be insufficient to tightly constrain any $\kappa$ deviations.

However, $(-++-)$ contributions, when used in conjunction with total cross section measurements, could provide important clarification of the source of a signal of non-standard physics. This is because the $(-++-)$ contribution information can provide a means of distinguishing 
between all the possible $\kappa_{\gamma}, \kappa_{Z}$ pairings that a total cross section measurement might indicate. In Chapter 4 we saw that a measurement of a non-standard cross sertion can, in general, be attributed to any of an infinite set of pairings of $\kappa_{\gamma}$ and $\kappa_{Z}$, these points lying on a contour of constant cross section. Although indistinguishable by a simple measurement of total cross section, these pairings will, in general, have very different characteristic helicity contributions. Thus, once a cross secticn measurement had indicated a non-standard $\kappa_{\gamma}, \kappa_{Z}$ pairing, the relative contributions of the helicity amplitudes to the total cross section could differentiate between the set of possible pairings responsible. We illustrate this idea in Figure (5.6). The $\pm 2 \sigma$ contour , corresponding to a cross section of $0.035 \mathrm{pb}$ ( compared to the Standard Model prediction $\sigma_{S M}=0.034 \mathrm{pb}$ ), is shown for the $\mu^{+} \tau^{-}$channel at $500 \mathrm{GeV}$. Superimposed on this contour are the contributions of the $(-++-)$ amplitude, in percent, to the total cross section, for selected pairings of $\kappa_{\gamma}$ and $\kappa_{z}$. These percentages exceed the approximate statistical level of uncertainty, $0.65 \rightarrow 1.4$, and so should be experimentally determinable.

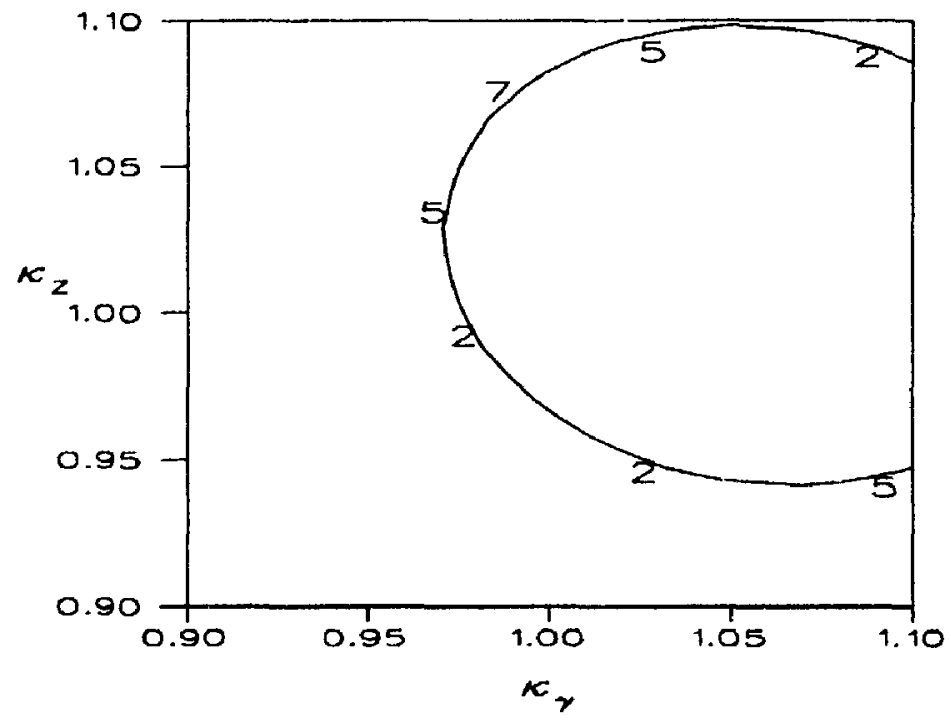

Tigure 5.6: Percentage contributions of $(-++-)$ amplitude to total cross section superimposed on $2 \sigma$ contour in $\kappa_{\gamma}$ and $\kappa_{Z}$ for $\mu^{+} \tau^{-}$channel at $\sqrt{s}=500 \mathrm{GeV}$.

Even with the additional information from the $(-++-)$ amplitude contributions, it will $n_{\sim} t$ be possible to uniquely determine the $\kappa_{\gamma}, \kappa z$ pairing responsible for a signal of non-standard total cross section. In general, there will still be four !:: sible pairings, determined by the intersection of the diagonal-iike helicity contribution contou.. .. ith the circular contours of the total cross 
section analysis.

We see that the shape of the 3-D surface for the contribution of the $(-++-)$ implitude, as it determines the nature of the contour lines, is critical for the differentiation of constant iross section pairings of $\kappa_{\gamma}$ and $\kappa_{z}$. More accurately. what is critical is that the contours it detines have a different topography than those of the total cross section, see Figures (4.6) ind (-1.7). If a helicity amplitudes' percent contribution contours followed the shape of the total iross section contours, then the pair discrimination ability would be lost: all pairings with constant rross section would have identical helicity contributions as well. It is the intersections of the contosurs that are essential; they reflect the fact that total and polarized cross section studies access very different information about the couplings $\kappa_{\gamma}$ and $\kappa_{z}$. By combining the constraints on $\kappa_{\gamma}$ and $\kappa_{Z}$ from both types of analysis, the sum, as reflected in the achievable limits on the couplings, is much greater than its parts. 


\section{Chapter 6}

\section{Form Factor Modification of Anomalous Couplings}

The high energy behaviour of the Standard Model cross sections for the four lepton production processes considered (cross section goes as $\frac{1}{3} \ln s$ as $s \rightarrow \infty$ ) is a consequence of the delicate cancellations that occur between the various contributions. For instance, for the process $e^{+} e^{-} \rightarrow$ $W^{+} W^{-}$(the three diagrams of which are a subset of those for the processes we consider), each of the six "component" cross sections (three diagrams plus interference terms) individually diverge at high energy. Their sum however, respects unitarity and is well behaved at asymptotic energies. The degree of precision necessary in the cancellation is demonstrated in Figure (6.1), where both the s-diverging neutrino exchange contribution from Figure (3.4a) and the asymptotically wellbehaved total cross section are shown as a function of energy.

The Standard Model cancellations are a physical manifestation of the underlying symmetry of the $S U(2)_{L} \times U(1)$ gauge structure. Indeed, the cancellations are destroyed if we allow the couplings of the theory to stray from their values prescribed by the gauge structure. Thus we saw how, for the four lepton production processes we have examined, non-standard pairings of the triple boson vertice couplings $\kappa_{\gamma}$ and $\kappa_{z}$ resulted in a cross-section that rose with energy (very slightly for the small deviations we consider here), see Figure (4.1).

It is precisely this behaviour that, at intermediate energies such as we considered in the previous Chapters, produces a discrepancy between the Standard Model cross section and that obtained with non-standard $\left(\kappa_{\gamma}, \kappa_{z}\right)$, and so provides a possible signal for just such "new physics". 


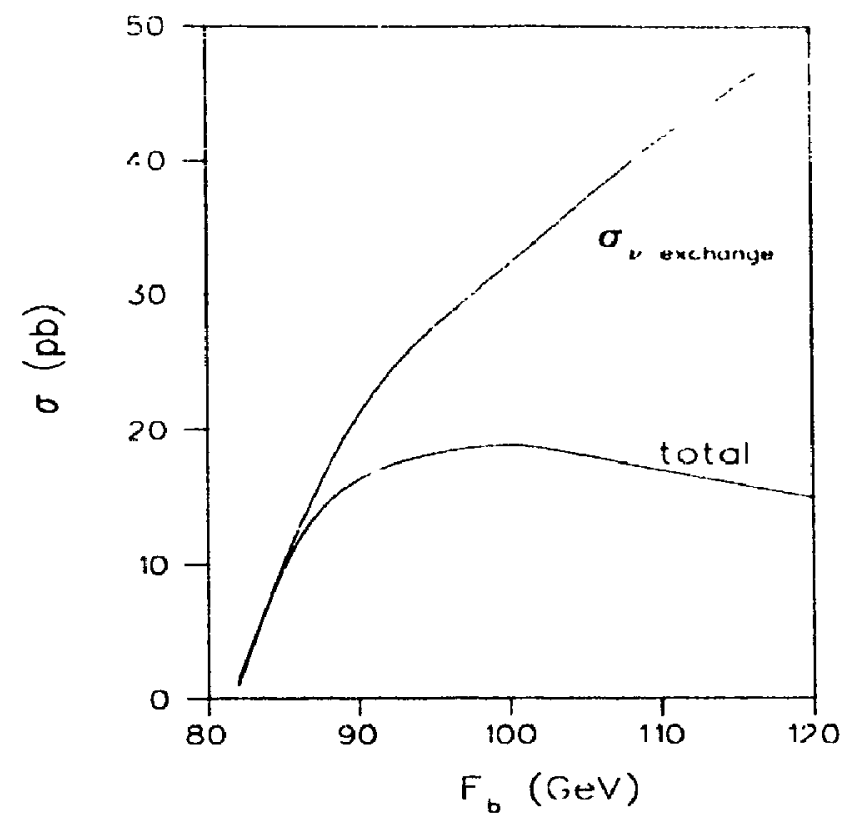

Figure 6.1: Cross-section as function of beam energy for the $e^{+} e^{-} \rightarrow W^{+} W^{-}$process. The total cross-section and neutrino contribution curves are shown. Figure is taken from "ECFA Workshop On LEP200", CERN 87-08.

If however, we require our cross-sections to satisfy the requirements of unitarity, then they must eventually "turn over" and, ultimately, decrease at asymptotic energies. This asymptotic requirement does not preclude the possibility of non-standard cross-seciions at intermediate energies, just that, as $s \rightarrow \infty$, we regain the Standard Model tehaviour.

More exactly, if we postulate that the deviations of $\kappa_{\gamma}$ and $\kappa_{Z}$ are caused by some new physics operating at an energy scale $\Lambda$, then we require that any deviation from the SM behaviour disappear as $s / \Lambda^{2} \rightarrow \infty$. Equivalently, we can require that the couplings $\kappa_{\gamma}$ and $\kappa_{Z}$ assume their gauge values of unity when $S / \Lambda^{2} \rightarrow \infty$. This was the approach taken by Baur and Doncheski [68] in their analysis of the $\gamma W W$ vertex through the processes $e^{ \pm} p \rightarrow \nu \gamma X$. To ensure trete-level unitarity, they parametrized the deviations $a=\Delta \kappa, \Delta \lambda$ as

$$
a\left(q_{W}^{2}, \bar{q}_{W}^{2}\right)=\frac{a_{0}}{\left(\left(1+q_{W}^{2} / \Lambda^{2}\right)\left(1+\bar{q}_{W}^{2} / \Lambda^{2}\right)\right)^{n}}
$$

where $a_{0}$ is considered to be the "low-energy" deviation, independent of energy and $q_{w}$ and $\bar{\eta}_{W}$ are the momenta of the two W's in their process. The exponent $n$ is a measure of how quickly 
$\Delta \kappa$ or $\Delta \lambda$ is suppressed; those authors assumed $n=1$. The scale $\Lambda$ represents the energy at which new physics becomes important in the weak boson sector and was set at $\Lambda=1 \mathrm{TeV}$. We see that the deviations $\Delta \kappa$ and $\Delta \lambda$ disappear for either or both of $\kappa, \lambda$ assuming their gauge values, or $q_{W}^{2}, \vec{q}_{W}^{2}>>\Lambda^{2}$. By parametrizing the deviations as energy dependent form factors in this manner, the authors ensured that, even for non-standard couplings $\kappa, \lambda$, they reproduce the required asymptotic energy behaviour.

The requirements of tree-level unitarity at asymptotic energies also place limits on possible deviations at lower energies, ie. for given values of $n$ and $\Lambda$, there will be a maximum possible $a_{0}$. Baur and Zeppenfeld [40], examined the constraints on the couplings of the most general three boson vertex from a partial wave unitarity analysis of the vector boson pair production amplitude. The limits they obtained for $\Delta \kappa_{\gamma}$ and $\Delta \kappa_{Z}$ were determined with the assumption that the deviations had the form $(\theta(x)$ is the step function)

$$
a\left(q^{2}, \bar{q}^{2}, P^{2}\right)=a_{0} \theta\left(\Lambda^{2}-q^{2}\right) \theta\left(\Lambda^{2}-\bar{q}^{2}\right) \theta\left(\Lambda^{2}-P^{2}\right)
$$

where $q, \bar{q}$, and $\mathbf{P}$ are the momenta of the $W^{+}, W^{-}$, and neutral vector boson, respectively. Their assumption is that the anomalous coupling is constant for $\sqrt{s}<\Lambda$, and then drops abruptly to zero at $\sqrt{s}=\Lambda$. Not surprisingly, bounds on $\Delta \kappa, \Delta \lambda$ derived from unitarity considerations depend explicitly on the assumptions made for the functional form and scale $\Lambda$ of the form factor. With the alternative form they assumed for the form factor, Eq. (6.1), Baur and Doncheski determined upper limits for the low-energy deviations of

$$
\begin{aligned}
& n=1 \quad\left|\Delta \kappa_{\gamma}\right| \leq 1.9 \mathrm{TeV}^{2} / \Lambda^{2} \\
& n=2 \quad\left|\Delta \kappa_{\gamma}\right| \leq 7.6 \mathrm{TeV}^{2} / \Lambda^{2}
\end{aligned}
$$

where $\Lambda$ is measured in TeV. With $n=2$, the deviations fall off faster with energy, thus the low energy deviations are less constrained.

Other authors have investigated such form factor corrections to the Standard Model gauge boson sector. For instance Rizzo [69] modified the propagators and triple boson vertices of the SM through inclusion of simple unitarity respecting form factors, as follows.

$$
\begin{aligned}
\frac{1}{p^{2}-M^{2}} & \rightarrow \frac{1}{p^{2}-M^{2}}\left(1+\frac{\lambda p^{2}}{\Delta^{2}}\right)^{-1} \\
\Gamma_{\mu \nu \gamma} & \rightarrow \Gamma_{\mu \nu \gamma}\left(1+\frac{s}{\Delta^{2}}\right)^{-1}
\end{aligned}
$$


where $\lambda= \pm 1$ such that $\lambda p^{2}>0$. Their interest was in the parameter 1 . the scale at which the gauge bosons might exhibit non-standard behaviour, attributed by the althors to possible boson compositeness. Their approach is fundamentally different from that outlined previously. Instead of attributing any signal of new physics to a specific origin. eg. non-standard $\mathrm{n}_{1}$, they simply parametrize any such signal as being due to some unknown source, operating at the sciale $\Lambda$. They therefore determine limits on $\boldsymbol{\Lambda}$, the scale of new physics, and not on any individual parameter that might signal such new dynamics.

We follow the approach of Baur and Doncheski; we attribute any non-standard cross-section to an intermediate energy effect of the unitarity respecting form factor $\Delta \mathrm{N} v$, whose form will be similar to Eq. (6.1). For low energies, if the scale $\Lambda$ is in the range $0.1<\Lambda<1.0$ TeV, the form factor correction would be neglible. At intermediate energies where $\sqrt{s} \sim \Lambda$, the "effective" deviation $\Delta \kappa_{V}$ could be substantially different than the "low energy" ( $\left.\kappa_{V}-1\right)$. The resultant non-standard cross-section could then be appreciably smailer (the form factor effect is to always give a smaller effective $\Delta \kappa v$, and thus a smaller deviation in the cross-section) than oblained without form factor correctons. Our aim in this Chapter is to determine how this proposed asymptotic behaviour will impact on the physics at intermediate energies, specifically whether or not the limits on $\Delta \kappa_{V}$ of the previous Chapters will be changed.

To demonstrate these ideas, and the nature of the results, we describe here how the form factor corrections were introduced into the $\mu^{+} \tau^{-}$channel, it being the simplest of the processes considered. For the $\mu^{+} \tau^{-}$channel, the sensitivity to $\kappa_{V}$ arises from the two s-channel neutral boson mediated $\mathrm{W}$-pair production diagrams of Figure (3.1), where the invariant masses of the two W's are $p^{2}$ and $q^{2}$. We parametrize the energy dependence of the deviations $\Delta \kappa v$ as

$$
\Delta \kappa V=\frac{(\kappa V-1)}{\left(1+p^{2} / \Lambda^{2}\right)^{n}\left(1+q^{2} / \Lambda^{2}\right)^{n}}
$$

With the deviations $\Delta \kappa_{V}$ thus modified, we ensure that they disappear for either or any of $\kappa_{V}=1, p^{2}, q^{2} \gg \Lambda^{2}$ ( $\Lambda$ is the scale at which the proposed non-standard physics will manifist itself). We explore the consequences of values for $\Lambda$ ranging from $0.5 \rightarrow 1 \mathrm{TeV}$ and allow for various values for the parameter $\mathrm{n}$. We show in Figure (6.2) the cross-section as a function of energy for various values of $\Lambda$ and $n$. The nor - standard curves all have $\kappa_{\gamma}=\kappa_{Z}=0.9$. 

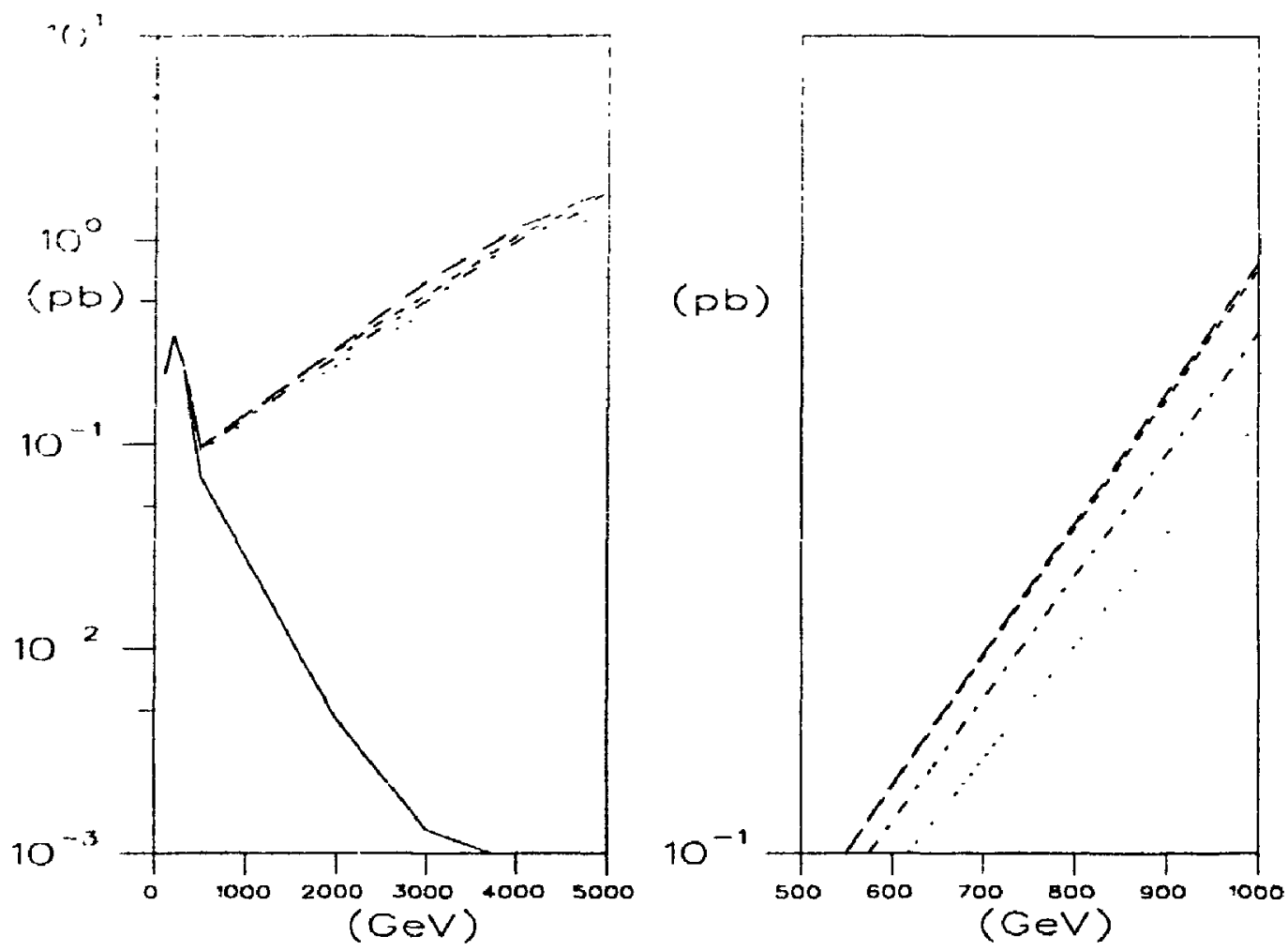

Figure 6.2: Gross-section as function of energy for $\mu^{+} \tau^{-}$process with various choices for $\Lambda$ and $n$. The right-hand figure is a magnified view of the energy range $500-1000 \mathrm{GeV}$. The solid line is that of the SM and the long dashed line that of $\kappa_{\gamma}=\kappa_{z}=0.9$, with no form factor modification. The other curves all have the same anomalous values for $\kappa_{\gamma}$ and $\kappa_{Z}$, but have, respectively, $\Lambda=1000 \mathrm{GeV}, \mathrm{n}=1$ (short dashed line), $\Lambda=500 \mathrm{GeV}, \mathrm{n}=1$ (dot-dashed line), and $\Lambda=500$ $\mathrm{GeV}, \mathrm{n}=2$ (dotted line).

The effect of finite, non-zero $\Lambda$ is to, over the energy zange shown, slow the rate of increase of cross-section with energy. Also, by comparing the two curves for $\Lambda=500 \mathrm{GrV}, \mathrm{n}=1$ and $n=2$, we verify that the effect of the larger exponent is to produce a more slowly rising cross-section. Not apparent from the limited scale of this plot is the ultimate "turn over" and eventually decreasing $\sigma$ at asymptotic energies. Although not explicitly demonstrated in the curves (the non-standard curves still show an increasing cross section) we verified that this did ultimately occur.

The three form-factor modified curves closely approximate the "no form factor" curve. At $\sqrt{s}=500 \mathrm{GeV}$, the cross-sections , . we different cases of form factor modification differ from the "no-form factor" anomalous value by approximately $1 \%$. Since the sensitivity to anomalous 
couplings is proportional to $\sigma^{-\frac{1}{2}}$. such a small modification of the tun-st andard cross-section will not change the previously deternined limits on $\kappa_{2}$ and $n z$. At the higher energy of $\sqrt{s}=1000$ $\mathrm{GeV}$, the form factor modifications can result in an anomalous cross-section approximately : \% lower than with no form factor modifiaction: this effect is however too small to appreriatly change the sensitivity to the vertex couplings.

At the center of mass energies we considered in the previous (hapters. 500 ( iev and 1 liev, we therefore see that, for larger values of $\Lambda$, the form factor modification will produce no signilicaut change in the non-standard cross-sections. Thus the limits we determined there for $\kappa_{\gamma}$ atul $\mathrm{n}_{2}$, which depend on the discrepancy between the non-standard and SM cross-sections, will not. be appreciably changed. For such values of $\Lambda$, the effect of varying the exponent $n$ is insigniticant.

We get similar results for the other channels, $\mu^{+} e^{-}, \mu^{+} \mu^{-}$, and $\rho^{+} p^{-}$. We modify the deviations $\Delta \kappa_{V}$ as before, but now account for the different momenta appearing at the triple boson vertex in the various channels. For instance, the $e^{+} e^{-}$process includes contributions from all the triple boson vertex diagrams, Figures 3.1, 3.3, and 3.5, in each of which the $W$ propagators have different momenta. It was generally observed that, if the new physics scalc parameter $\Lambda$ exceeded approximately $500 \mathrm{GeV}$, then the limits for $\Delta \kappa V$ previously obtained without accounting for possible form factor corrrcctions would be unchanged by doing so.

Barger and Reno [70] performed a similar form factor analysis of anomalous 'TBV contributions to $p p$ and $p \bar{p} \rightarrow W^{ \pm} \gamma X$ at the Tevatron $(\sqrt{s}=1.8 \mathrm{TeV})$ and LHC: $(\sqrt{s}=14 \mathrm{TrV})$. 'They found that, for the Tevatron, the event rates are nearly equal with and without form factors; the differences in number of events are not statistically significant in terms of placing limits on $\Delta \boldsymbol{r}$. At the LHC, the form factor results are measurably lower than those without the form fartor modification, with consequently looser bounds on $\kappa_{V}$ being predicted.

If we define $\Delta \kappa_{V}^{*}$ through the relation

$$
\Delta \kappa v=\frac{\Delta \kappa_{V}^{*}}{\left(1+p^{2} / \Lambda^{2}\right)\left(1+q^{2} / \Lambda^{2}\right)}
$$

It is apparent that we can achieve the same $\Delta \kappa v$, and corresponding total cross-scrtion, through combining a small $\left|\Delta \kappa_{V}^{*}\right|$ with a large $\Lambda$, or vice versa. 'Thus, for the $\mu^{+} \tau^{-}$channel at. $\sqrt{S}=1000 \mathrm{GeV}$, we get approximately the same cross-section for $\Delta \boldsymbol{x}_{V}^{*}=-0.1$ and $\Lambda=100$ $\mathrm{GeV}$ as we do for $\Delta \kappa_{V}^{*}=-0.01$ and no form factor $(\Lambda=x)$. This implies that, for intermediatr. energies, the requirements of unitarity at asymptotic energies place restrictions on low enrrgy $\Delta \kappa_{V}^{*}$ that scale with $\Lambda$; the greater the value for $\Lambda$, the stronger the restriction on possiblo 
$\left|\Delta x_{i}\right|$ at intermediate energies where $\sqrt{s}<\Lambda$. This is of course the significance of the limits of Baur and Doncheski, their limits for the deviations also imply that the larger $\Lambda$ is, the more constrained are the low energy deviations.

We ran also relate the specific unitarity limits of Baur and Doncheski to our results. We found that, if $\Lambda$ was equal to or greater than approximately $500 \mathrm{GeV}$, then the liugits on $\Delta \kappa V$ we determined in previous Chapters would not, be appreciably changed by the form factor corrections. We see, from the limits derived by Baur and Doncheski, that a value of $500 \mathrm{GeV}$ for $\Lambda$ corresponds to a limit on $\Delta \kappa_{V}$ of the order $7-8$, if $n=1$. The D0 collaboration [54] reports a value of $-2.3<$ $\Delta \kappa<2.2$, roughly corresponding to $\Lambda=900 \mathrm{GeV}$. This supports our assumption that the scale parameter $\Lambda$ is larger than $500 \mathrm{GeV}$.

The connection between $\Delta \kappa_{V}$ and $\Lambda$, visible in our results, and expressed more rigorously in the limits of Baur and Doncheski, could have important implications for the sensitivity requirements of future high energy facilities. If an experiments' expected level of precision for the deviations exceeds the unitarity upper limit, then no useful limits could be determined. Thus, an experiments' minimum uncertainty in $\Delta \kappa_{V}$ corresponds to a maximum "allowable" $\Lambda$, anything greater places too severe a constraint on low-energy $\Delta \kappa_{v}$. To probe the couplings $\kappa_{\gamma}$ and $\kappa_{Z}$ at the level of a few percent, the scale of precision predicted necessary by SM loop calculations and the various extensions to the SM, the unitarity limits suggest that $\Lambda$ must not exceed approximately $10-20 \mathrm{TeV}$. 


\section{Chapter 7}

\section{CP Violating Triple Vertex Contribution}

The Standard Model of the electroweak interactions is unable to satisfactorily explain the origin of CP violation. Symmetry under the CP operation assumes that the physics of a process should bu unchanged by simultaneous interchange of particle and antiparticle, and reversing the diruction of momenta. The fundamental nature of this symmetry, and the implications that any violation has, as a result of the CPT theorem, for the other fundamental symmetry of time reversal invariance, demand a greater understanding of the underlying mechanism. Our understanding is limitru, in part, by the lack of experimental information on the subjec.. Since it was first discovered [71] in 1964 in the neutral $K^{-0}-\bar{K}^{0}$ system, other searches have produced only negative constraints. For instance, upper bounds on the neutron electric dipole moment iraply that any (:P violating contribution to the WWy vertex should be of order $10^{-4}$ or less [72]. An electric dipole moment for the neutron would arise from an asymmetry between positive and negative charge clouds relative to the spin $\sigma$. Such a charge asymmetry violates ' $T$ reversal invariance, and by the (;P' $T$ theorem, CP invariance. The detection of new CP violating (CPV) effects in sectors baysud the neutral $\mathrm{K}$ system would provide exciting possibilities for new insights into the underlying dynamice of CP violation, just as a negative search could further constrain its existence in these different sectors. Future high-energy colliders, with their ability to probe these unexploresl regions, offer exciting potential for investigating possible new sources of CP violation. Berauss: the SM strongly limits any CPV in high-energy collisions, any evidence of such will be a window 
into the physies beyond the Standard Model.

Within the $S U(2)_{L} \times U(1)$ frameuork of the SM. CP violation is only present via the Kisbayashi-Maskawa phase [73]. which afferts $e^{+} e^{-}$annihiliation inio $W^{+} W^{-}$pairs at two-loop order and higher, (because the CKM matrix belongs to the quark sector, there must be at leasi two loops in the SM to allow the quarks to contribute to a leptonic process). One-loop effects are however possible in ron-siandard theories. Even in these non-standard models, such as LeftRight symmetry, Supersymmetry, or theories with extra Higgs, where CPV can appear at one loop, it manifests itself in form factors for the thiee gauge boson vertex that are $10^{-2}$ or smaller due to the loop suppression [24]. Additionally, with the CP contribution to the $W W_{\gamma}$ vertex so severely constrained by, amongst others, the neutron electric dipole moment measurements. there are theoretical arguments [74] that give reason to doubt the existence of a CP violating $Z$ interaction. If a CP violating electromagnetic interaction is excluded, then a CPV ZWW yertex contribution would imply that the vector boson interactions must violate $\mathrm{SU}(2)$ weak isospin symmetry. There is however good evidence for the validity of this SU(2) symmetry.

With Standard Model CP violating contributions severely constrained by experiment, and small theoretical expectations for any non-standard one-loop contributions, we must expect any CF violation in the triple boson vertices to be a small effect. Nevertheless, to rule out or further constrain the existence of such CP violation, it will still be invaluable to perform the search. Most previous work on the search potential of various processes to anomalous triple boson vertices, motivated by the stringent experimental bounds, has ignored the possibility of a CPV contribution. The research that has accounted for CPV has largely concentrated on the process $e^{+} e^{-} \rightarrow W^{+} W^{-}$, with subsequent leptonic decay of the $W$ bosons [20,37]. As previously determined, this approximation to the full four lepton production process quickly becomes unsatisfactory at energies beyond LEP2. We therefore believe that our treatment of the complete four lepton channels, including those contributions additional to $W$-pair production. will give more realistic limits on a CPV contribution to the triple boson vertices.

In Section 7.1 we discuss the restrictions on the CP-violating couplings that the CPT theorem imposes. We determine detection limits on the couplings $\tilde{\kappa}_{\gamma}$ and $\tilde{\boldsymbol{x}}_{Z}$ in Section 7.2, through both total cross-section measurements and $a x^{2}$ analysis of an angular distribution. We explore the sensitivity of certain experimental dist ributions to non-zero CP-violating couplings in Section 7.3. We examine asymmetries in certain CP-odd variables as an additional indicator of a CP-violating contribution in Section 7.4. 


\subsection{CP violation in Triple Gauge Boson Vertices}

If we allow for the possibility of ('PV' in the there boson vertex. the momentum spatio aflientive vertex ran be expressed as follows

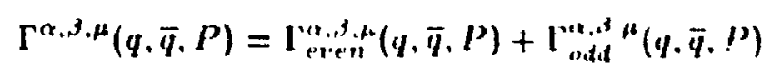

where $\Gamma_{\text {even }}$ contains the CP-even couplings and $\mathrm{I}_{\text {odd }}$ is the ( $\mathrm{P}$-odd pioce.

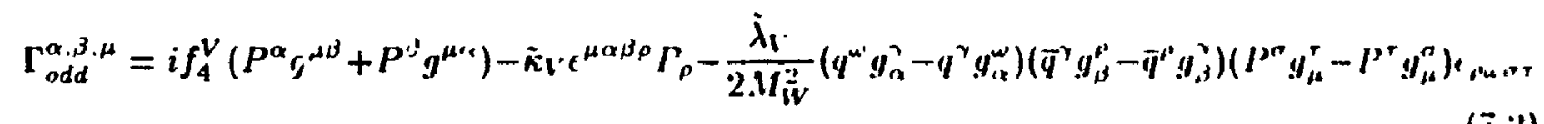

wher $\epsilon$ we follow tie conventions of Hagiwara et al. [20], $V^{\circ}=\gamma, Z$ and all the momenta are defined as entering the triple vertex. The Standard Mode! predicts for these ('P-odd form factors, at. tree and one-loop .evel,

$$
f_{4}=r i \cdot=\dot{\lambda v}=0
$$

For extensions to the SM, these three form factors $f_{4}, \kappa_{\bar{V}}$, and $\overline{\lambda_{i}}$ can, depending on the model and the kinematics, have both real and imaginary parts. The effects of the two different poisibilities can be separated by examining the consequences of the (PT theorem and the unitiarily condition. The CPT theorem postulates the invariance

$$
<f|M| i>=<C P T(i)|M| C^{\prime} P T(f)>
$$

where $\mid C P T(j)>$ represents the state $\mid j>$ with its quantum numbers and kinematic variabless transformed by CPT (the CP transformation with an additional flip of the time arrow). It is very difficult to directly check this symmetry because it requires the interchange of initial and final states. It is more convenient to define a pseudo time reversal transformation, $\tilde{T}$, that transforms the kinematic observables of the initial and final state as does ' $T$, but does not interchange thr. initial and final stotes, as $\mathrm{T}$ would.

In the Born ap sroximation, unitarity of the S-matrix implies that the transition matrix, $M$. is hermitian. Thus, in the Born approximation, M satisfies

$$
M_{s}=M_{f}^{*}
$$


And so, with a hermitian transition matrix, the CPT theorem reduces to

$$
<f|M| i>=<C P \tilde{T}(f)|M| C P \tilde{T}(i)>
$$

The CP'T theorem therefore provides a check on the hermiticity of the transition matrix $M$. Non-hermiticity of $\mathbf{M}$, which is due to contributions beyond Born in which intermediate states ran be on-shell, will manifest itself in violations of $C P \tilde{T}$.

We approximate our complete process of four lepton production by $\mathrm{W}$ pair production for the purpose of discussion. If we define $A_{\lambda, \bar{\lambda}}$ as the tree level SM contribution to the transition matrix (with basis $(-, 0,+)$, the helicities of the W's) and $\delta A_{\lambda, \bar{\lambda}}$ as the deviation due to the CP violating couplings, $\delta A_{\lambda, \bar{\lambda}}$ can be expressed ac [37]

$$
\delta A_{\lambda, \bar{\lambda}}=\left[\begin{array}{ccc}
-i\left(\beta^{-1} \tilde{\kappa}+4 \gamma^{2} \beta \tilde{\lambda}\right) & -i \gamma\left(f_{4}+\beta^{-1} \tilde{\kappa}\right) & 0 \\
-i \gamma\left(-f_{4}+\beta^{-1} \tilde{\kappa}\right) & 0 & i \gamma\left(f_{4}+\beta^{-1} \tilde{\kappa}\right) \\
0 & i \gamma\left(-f_{4}+\beta^{-1} \tilde{\kappa}\right) & i\left(\beta^{-1} \tilde{\kappa}+4 \gamma^{2} \beta \tilde{\lambda}\right)
\end{array}\right]
$$

The coefficients in the above are $\gamma=1 / 2 \sqrt{s} / m_{W}$ and $\beta=1-\gamma^{2}$. Under the $C P \tilde{T}$ transformation, we must have

$$
\delta A_{\lambda, \bar{\lambda}} \stackrel{C P \tilde{T}}{\rightarrow} \delta A_{-\bar{\lambda},-\lambda}^{*}
$$

'To check this, we look at $\delta A_{0,-}$, and demonstrate with the $f_{4}$ terms.

$$
\begin{array}{lll}
\delta A_{0,-} & = & i \gamma f_{4} \\
\delta A_{0,-} & \stackrel{C P \bar{T}}{\rightarrow} & \left(\delta A_{+, 0}\right)^{*} \\
\delta A_{0,-} & \stackrel{C P}{\rightarrow} & \left(-i \gamma f_{4}\right)^{*} \\
\delta A_{0,-} & \stackrel{C P}{\rightarrow} & i \gamma f_{4}^{*}
\end{array}
$$

$C P \dot{T}$ therefore requires

$$
i \gamma f_{4}=i \gamma f_{4}^{*}
$$

which requires $f_{4}$ to be real. An imaginary component of the $f_{4}$ form factor (and likewise for $\tilde{\kappa}$ and $\bar{\lambda}$ ) will therefore break the $C P \dot{T}$ symmetry, and so parametrizes the non-hermiticity of the transition matrix, the hallmark of beyond Born final state interactions. Such effects are small in a weakly coupled theory such as the SM, and so in what follows we concentrate on the case 
where all form factors are real.

Of the three CP-odd couplings, $\bar{k}$ and $\bar{\lambda}$ are C-even and P-odd, $f_{4}$ is P-pven and (c-odd. Thus a non-zero $f_{4}^{\gamma}$ is forbidden by electromagnetic gauge invariance. With $f_{A}^{\gamma}=0$, the existence of a non-zero $f_{4}^{Z}$ would imply that the $W$ boson interactions intrinsically violate the $S(I)$ weak-isospin symmetry. There is, however, good empirical evidence for the validity of this symmetry. Accordingly, we assume also that $f_{4}^{Z}=0$ and restrict ourselves to the C'P-odd couplings $\tilde{\kappa}_{\gamma}, \tilde{\kappa}_{z}, \bar{\lambda}_{\gamma}$, ard $\tilde{\lambda}_{z}$. Also, in the analysis of these CP-odd couplings, we restrict the (:P-even couplings to their SM values, $\kappa V=1$ and $\lambda_{V}=0$.

\subsection{Sensitivity Limits on $\tilde{\kappa}_{V}$ and $\bar{\lambda}_{V}$}

\subsubsection{Total Cross-Section Limits}

We determine limits on $\tilde{\kappa}_{V}$ and $\bar{\lambda}_{V}$ as we did for the CP-even $\kappa_{\gamma}$ and $\kappa_{z}$. Varying only one of the four CP-odd couplings at a time, we fit parabolas to the dependence of the total cross-section on each of the couplings. The critical cross-section, that determined by assuming a statistical error of $\sqrt{\sigma_{S M} / L}$ about $\sigma_{S M}$, once substituted into the equations of these parabolas, determined the corresponding values for the couplings $\tilde{\kappa}_{\gamma}, \tilde{\kappa}_{Z}, \tilde{\lambda}_{\gamma}, \tilde{\lambda}_{Z}$. This procedure was performed for pach of the couplings, through eacl, of the four lepton channels, at the center of mass energies of $\sqrt{s}=500 \mathrm{GeV}$ and $1 \mathrm{TeV}$. Since the CP-odd couplings will contribute for the most part to first order quadratically, we make the approximation that the limits $\Delta<0$ and $\Delta>0$ are equal. These limits are listed below in Tables 7.1 and 7.2. 
Table $7.1: 95 \%$ C.L. bounds on non-standard couplings $\tilde{\kappa}_{V}$

\begin{tabular}{|c|c|c|c|c|c|}
\hline Process & $\sqrt{s}$ & \multicolumn{4}{|c|}{ Sensitivity Limits } \\
\hline \multirow[t]{2}{*}{$\mu \tau$} & $500 \mathrm{GeV}$ & $\overline{\mid \tilde{\kappa}_{\gamma}}$ & $<0.19$ & $\overline{\mid \tilde{\kappa}_{Z}}$ & $<0.17$ \\
\hline & $1000 \mathrm{GeV}$ & $\overline{\boldsymbol{\kappa}}_{t}$ & $<0.13$ & $\overline{\bar{\kappa}_{Z}}$ & $<0.12$ \\
\hline \multirow[t]{2}{*}{$\mu e$} & $500 \mathrm{GeV}$ & $\mid \tilde{\boldsymbol{\kappa}}_{\gamma}$ & $<0.18$ & $\overline{\tilde{\kappa}_{Z}}$ & $<0.19$ \\
\hline & $1000 \mathrm{GeV}$ & $\overline{\bar{\kappa}_{\gamma}}$ & $<0.14$ & $\overline{\bar{\kappa}_{Z}}$ & $<0.15$ \\
\hline \multirow[t]{2}{*}{$\mu \mu$} & $500 \mathrm{GeV}$ & $\tilde{\kappa}_{\gamma}$ & $<0.26$ & $\tilde{\tilde{\kappa} z}$ & $<0.20$ \\
\hline & $1000 \mathrm{GeV}$ & $T \bar{\kappa}_{\gamma}$ & $<0.23$ & $\tilde{\boldsymbol{\kappa}}_{\boldsymbol{Z}}$ & $<0.10$ \\
\hline \multirow[t]{2}{*}{$\overrightarrow{e e}$} & $500 \mathrm{GeV}$ & $\overline{\boldsymbol{\kappa}}_{\gamma}$ & $<0.15$ & $\tilde{\boldsymbol{\kappa}}_{\boldsymbol{Z}}$ & $<0.16$ \\
\hline & $1000 \mathrm{GeV}$ & $\mid \tilde{\boldsymbol{\kappa}}_{\gamma}$ & $<0.14$ & $\overline{\tilde{\kappa} z}$ & $<0.12$ \\
\hline
\end{tabular}

Table 7.2: $95 \%$ C.L. bounds on non-standard couplings $\tilde{\lambda}_{V}$

\begin{tabular}{|c|c|c|}
\hline Process & $\sqrt{s}$ & Sensitivity Limits \\
\hline \multirow[t]{2}{*}{$\mu \tau$} & $500 \mathrm{GeV}$ & $\left|\lambda_{\gamma}\right|<0.0052 \quad\left|\lambda_{z}\right|<0.0047$ \\
\hline & $1000 \mathrm{GeV}$ & $\left|\lambda_{\gamma}\right|<0.00047 \quad\left|\lambda_{z}\right|<0.00043$ \\
\hline \multirow[t]{2}{*}{$\mu e$} & $500 \mathrm{GeV}$ & $\left|\hat{\lambda}_{y}\right|<0015$ \\
\hline & $1000 \mathrm{GeV}$ & $\left|\lambda_{y}\right|<0.0014$ \\
\hline \multirow[t]{2}{*}{$\mu \mu$} & $500 \mathrm{GeV}$ & $\left|\bar{\lambda}_{\gamma}\right|<0.0038$ \\
\hline & $1000 \mathrm{GeV}$ & $\left|\lambda_{z}\right|<0.9014$ \\
\hline \multirow[t]{2}{*}{$\overrightarrow{e e}$} & $500 \mathrm{GeV}$ & $\left|\lambda_{\gamma}\right|<0.004$ \\
\hline & $1000 \mathrm{GeV}$ & $\left|\hat{\lambda}_{y}\right|<0.0014$ \\
\hline
\end{tabular}

The achievabie limits on $\tilde{\kappa}_{V}$ from Table 7.1 are quite loose, especially compared to those evaluated for the CP-even $\kappa_{\gamma}$ and $\kappa_{Z}$, see Chapter 4. The differences in sensitivity can be attributed to the relative phase difference between the CP-odd and CP-even contributions. We write the modified amplitude as the sum of the Standard Model contribution and the nonstandard, itself composed of CP-even pieces and CP-odd pieces.

$$
M=M_{S M}+M_{N S M}^{C P \text { even }}+i\left(M_{N S M}^{C P-o d d}\right)
$$

The phase difference between the CP-even and CP-odd contributions ensures that interference between the two is minimal. Thus the CP-odd couplings contribute predominantly to first order 
quadratically. Neglecting the small interference effects, the total cross-section can be written as.

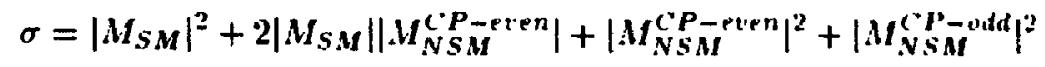

The contributions of the non-standard CP-even couplings can interfere with the Standard Model amplitude, thus a small coupling can have appreciable effects. 'The (P-odd couplings however, because they predominantly contribute to first order only quadratically, do not produce sizable interference effects. Thus, while $\kappa_{V}$ and $\tilde{\kappa}_{V}$ share the same asymptotic energy behaviour, contributions rise like $\sqrt{s}$, the interference between the $\kappa_{V}$ terms and the Standard Moctel contribution, missing for the $\bar{\kappa}_{V}$ contributions, ensures that the total cross-section is much more sensitive to the CP-even couplings. While the contributions of the $\bar{\lambda}_{V}$ terms also contribute to first order only quadratically, they have a much more promising high energy behaviour. This is because they rise with energy like $s$, and not $\sqrt{s}$ as does $\tilde{\kappa}_{v}$. The limits on $\dot{\lambda}_{v}$, as listed in Table 7.2, are consequently much tighter than those for $\tilde{\kappa}_{V}$, Table 7.1. Indeed, some of the limits on $\tilde{\lambda}_{V}$ from the different channels are of the same scale as predicted for these couplings by the various "beyond-Standard" models, specifically the $\mu^{+} \tau^{-}$process at the higher energy. Also, they approach the level of precision predicted necessary by neutron electric dipole moment measurements.

\subsection{2 $\chi^{2}$ Analysis}

The derived limits from Tables 7.1 and 7.2 are fully "integrated", ie. they are obtained from the sensitivity of the total cross-sections to non-standard couplings. However, we have sern previously that the sensitivity to non-standard couplings is generally not evenly distributed over the full phase space. Thus, any limits derived from total cross-section, with these different regions of sensitivity integrated over, are "blind" to this sensitivity localization. In Chapter 4 we explored the idea of taking advantage of this phenomena by making suitable cuts to mimimize the contribution of the non-sensitive regions, thereby hopefully improving the achievable limits on the non-standard couplings. However, as we saw, these regions of low sensitivity usually also contain the dominant contributions to the total cross-section; excluding them through ruts can seriously diminish the statistics and consequently, the derived limits. In addition, although these regions are relatively insensitive to the non-standard couplings, there is still "information" present, information that would be lost should the cuts be implemented. To prevent the loss of this information, and determine limits on the non-standard couplings that more arcurately 
acrount for the unevenly distributed sensitivity, we performed a $x^{2}$ analysis for these CP-odd rouplings.

To demonstrate the principle of a $x^{2}$ analysis, we consider a distribution where the sensitivity to $\tilde{n}_{V}$ and $\tilde{\lambda}_{V}$ is small where the differential cross-section is large, and large where the differential cross-section is small. The distributions in $\theta_{l-}, \theta_{l^{+}}$, the angle between the outgoing lepton(antilepton) and the beamline, are good examples. There, the dominant contribution of the t-channel neutrino exhange diagram, with no triple boson vertex dependence, makes the distributions peak at $\cos \theta= \pm 1$ respectively. We divide the phase space into bins, the choice of number and positioning of bins roughly determined by the regions of different sensitivity. We then define our $x^{2}$ test variable as

$$
x^{2}=\sum_{i}^{n}\left[\frac{\left(X_{i}-Y_{i}\right)^{2}}{\Delta_{i}^{2}}\right]
$$

where

$$
X_{i}=\left(\frac{d \sigma_{N S M}}{d \cos \theta_{l+}}\right)_{i}, Y_{i}=\left(\frac{d \sigma_{S M}}{d \cos \theta_{l+}}\right)_{i}
$$

and $\sigma_{N S M}$ and $\sigma_{S M}$ are the anomalous and standard cross-sections, respectively. $\Delta_{i}^{2}$ combines both statistical and systematic errors for the particular bin. ${ }^{1}$

$$
\Delta_{i}^{2}=\left(\Delta_{i}^{s t a t}\right)^{2}+\left(\delta^{s y z} Y_{i}\right)^{2}
$$

Where the cross-section is large, the sensitivity, and consequently the "variance" $\left(X_{i}-Y_{i}\right)^{2}$ is small. However, if $Y_{i}$ is large, then the statistics should be improved, and $\Delta_{i}^{2}$ will be relatively small. Conversely, for regions where the sensitivity is large, the variance $\left(X_{i}-Y_{i}\right)^{2}$ will be large, but so will the "error", due to the poorer statistics. By summing over these different regions, $\chi^{2}$ more democratically weights the contributions from regions of phase space with different levels of sensitivity, thus giving a truer estimate of the "deviation" of the non-standard cross-section

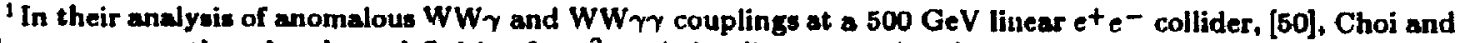
Schrempp argue that the above definition for $\chi^{2}$, as it implies uncorrelated systematic errors for the bins, is leas appropriate than their alternutive expresoion for $\chi^{2}$, involving a free normalization constant $f_{\text {norm }}$ and correlated bin by bin systematics. If we asume the simpler treatment of the systemmlics, and take the total crose-section for the $\mu \mathrm{T}$ channel at $500 \mathrm{GeV}$ of $0.068 \mathrm{pb}$ an example, the systematic error will dominate the atatistical if S.y' $2 \%$ or greater. Crude eatimates of the expected aystematics at the future high energy facilities vary from $2 \%$ (Choi and Schrempp's value ) to $5 \%$. It would therefore seem that proper treatment of these syatematica is crucial in determining realistic limits for the anomalous couplings. However, despite this, a comparison of ralculated $x^{2}$ for the two different treatments of the aystematics revealed insignificant differences between the two. Therefore, to simplify the analyais, we used the given expression for $x^{2}$, and not that of Choi and Sehrempp.
} 
from the standard.

The larger the magnitude of the non-standard couplings, the greater will be the terms $\left(F_{t}-Y_{i}\right)^{2}$, and consequently, the larger $y^{2}$ will be. By choosing a critical value for $l^{2}$, corresponding to a certain required Confidence Level, the magnitudes of non-standard couplings required to achieve this $\gamma_{C}^{2}$ can be determined. As was previously mentioned, the choire of the number of bins, and how phase space is apportioned amongst them.(the bins need not be the same width), is arbitrary. Because it is the $1^{2} / D . F$. that determines the form factor linits; and the Degrees of Freedom is given by D.F. = \# bins -1 , a large number of bins is not automatically preferred. We experimented with bin number and size, and generally found that, more important than the number of bins in optimizing the limits, was that the bins approximated the different. sensitivity regions, ie. a single bin shouldn't contain both high and low sensitivity regions of phase space.

$\chi^{2}$ was calculated from the $\theta_{l}$ - distribution as a sum over the following three bins, $(-.95 \rightarrow-.5),(-.5 \rightarrow 0)$ and $(0 \rightarrow .95)$. With 2 D.F, the $95 \%$ C.L. is $x^{2}=6.0$. We show in Tables 7.3 and 7.4 the limits on $\tilde{\kappa}_{V}$ and $\tilde{\lambda}_{V}$ corresponding to this $x^{2}$. The systematic error was assumed to be $5 \%$.

Table 7.3: $\chi^{2} 95 \%$ C.L. bounds on non-standard couplings $\tilde{\kappa}_{V}$. Limits are taken from $\gamma^{2}$ as function of $\tilde{\boldsymbol{\kappa}}_{\boldsymbol{V}}$.

\begin{tabular}{|c|c|c|c|}
\hline Process & $\sqrt{s}$ & \multicolumn{2}{|l|}{ Sensitivity Limits } \\
\hline \multirow[t]{2}{*}{$\mu \tau$} & $500 \mathrm{GeV}$ & $-0.17<\tilde{\kappa}_{\gamma}<0.16$ & $-0.14<\tilde{\kappa}_{Z}<0.12$ \\
\hline & $1000 \mathrm{GeV}$ & $-0.11<\tilde{\kappa}_{\gamma}<0.12$ & $-0.10<\tilde{\kappa}_{Z}<0.095$ \\
\hline \multirow[t]{2}{*}{$\mu e$} & $500 \mathrm{GeV}$ & $-0.19<\tilde{\kappa}_{\gamma}<0.18$ & $-0.16<\bar{\kappa}_{z}<0.15$ \\
\hline & $1000 \mathrm{GeV}$ & $-0.10<\bar{\kappa}_{y}<0.095$ & $-0.090<\tilde{\kappa}_{Z}<0.090$ \\
\hline \multirow[t]{2}{*}{$\mu \mu$} & $500 \mathrm{GeV}$ & $-0.22<\tilde{\kappa}_{\gamma}<0.21$ & $-0.18<\bar{\kappa}_{Z}<0.17$ \\
\hline & $1000 \mathrm{GeV}$ & $-0.21<\tilde{\kappa}_{\gamma}<0.19$ & $-0.090<\tilde{\kappa}_{Z}<0.085$ \\
\hline \multirow[t]{2}{*}{$\overline{e e}$} & $500 \mathrm{GeV}$ & $-0.13<\tilde{\kappa}_{\gamma}<0.12$ & $-0.12<\bar{\kappa}_{z}<0.12$ \\
\hline & $1000 \mathrm{GeV}$ & $-0.12<\vec{\kappa}_{\gamma}<0.11$ & $-0.10<\tilde{\kappa}_{Z}<0.09$ \\
\hline
\end{tabular}


Table 7.4: $\chi^{2} 95 \%$ C.L. bounds on non-standard couplings $\bar{\lambda}_{V}$. Limits are taken from $\chi^{2}$ as function of $\bar{\lambda}_{V}$.

\begin{tabular}{|c|c|c|}
\hline Process & $\sqrt{8}$ & Sensitivity Limits \\
\hline \multirow[t]{2}{*}{$\mu \tau$} & $500 \mathrm{GeV}$ & $-0.007<\lambda_{\gamma}<0.006 \quad-0.006<\lambda_{Z}<0.005$ \\
\hline & $1000 \mathrm{GeV}$ & $-0.00034<\lambda_{\gamma}<0.00031$ \\
\hline \multirow[t]{2}{*}{$\mu e$} & $500 \mathrm{GeV}$ & $-0.013<\bar{\lambda}_{y}<0.012 \quad-0.012<\bar{\lambda}_{z}<0.011$ \\
\hline & $1000 \mathrm{GeV}$ & $-0.001<\lambda_{\gamma}<0.0008 \quad-0.0008<\lambda_{z}<0.0007$ \\
\hline \multirow{2}{*}{$\mu \mu$} & $500 \mathrm{GeV}$ & $-0.0016<\bar{\lambda}_{\gamma}<0.0015 \quad-0.0014<\bar{\lambda}_{Z}<0.0013$ \\
\hline & $1000 \mathrm{GeV}$ & $-0.0013<\lambda_{z}<0.0011$ \\
\hline \multirow[t]{2}{*}{$e e$} & $500 \mathrm{GeV}$ & $-0.0035<\bar{\lambda}_{\gamma}<0.0036$ \\
\hline & $1000 \mathrm{GeV}$ & $-0.0014<\lambda_{\gamma}<0.0012$ \\
\hline
\end{tabular}

Comparing the limits from Tables 7.3 and 7.4 for the $\chi^{2}$ analysis to those limits from the analysis of the total cross-section, Tables 7.1 and 7.2 , we see that by accounting for the uneven sensitivity localization, the limits on $\tilde{\kappa}_{V}$ and $\tilde{\lambda}_{V}$ are improved by approximately $10 \%$, relative to those limits outained from a total cross-section analysis.

\subsection{Distributions as Indicators of CP violation}

A simple measurement of total cross-section will be unable to determine whether the source of an anomalous signal is CP respecting or CP violating. Perhaps the various experimental distributions can provide additional information on the source, either $\mathrm{CP}$ violating or respecting, of a new physics signal, once indicated by a non-standard cross-section. For the two cases of CPeven, $\kappa_{\gamma}=\kappa_{Z}=0.9$, and CP-odd, $\tilde{\kappa}_{Z}=1.0$, we show in Figures (7.1) and (7.2) the distributions in the angular variable $\theta_{\tau}$ - (where $\theta_{\tau-}$ is the angle between the outgoing $r$ - and the incoming $e^{-}$) and $M_{\mu \tau}$, the invariant mass of the outgoing lepton-antilepton pair, for the $\mu^{+} \tau^{-}$channel at $\sqrt{s}=500 \mathrm{GeV}$. 


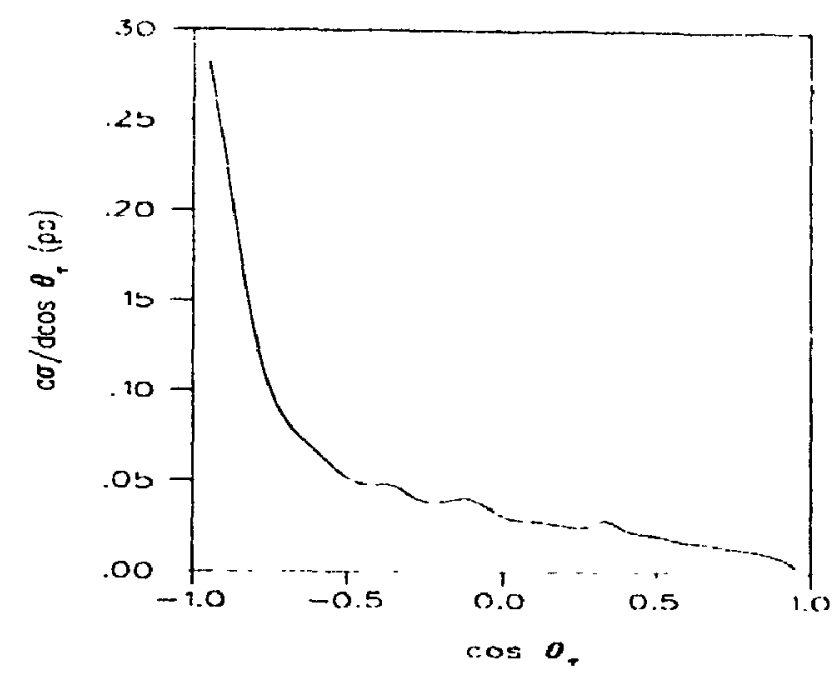

Figure 7.1: Differential cross-section with respect to $\cos \theta_{r-}$ for the two non-standard cases of CP-even $\kappa_{\gamma}=\kappa_{z}=0.9$ (solid line), and CP-odd $\tilde{\kappa}_{Z}=1.0$ (dotted line) for the $\mu^{+} \tau^{-}$channel at $\sqrt{s}=500 \mathrm{GeV}$. For comparison, the cross-sections for the two cases are $\sigma=0.11 \mathrm{pb}$ and $\sigma=0.14 \mathrm{pb}$ respectively.

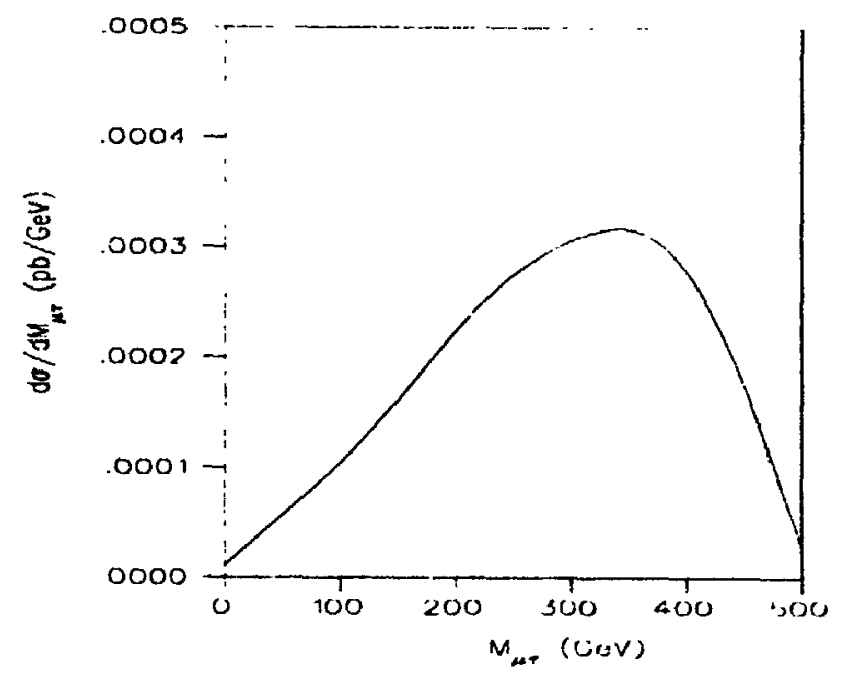

Figure 7.2: Differential cross-section with respect to $M_{\mu \tau}$ for the two non-standard cases of (:P. even $\kappa_{\gamma}=\kappa_{Z}=0.9$ (solid line), and CP-odd $\tilde{\kappa}_{Z}=1.0$ (dotted line) for the $\mu^{+} \tau^{-}$channel $u t$ $\sqrt{8}=500 \mathrm{GeV}$. For comparison, the cross-sections for the two cases are $\sigma=0.11 \mathrm{pb}$ and $\sigma=0.14$ $\mathrm{pb}$ respectively. 
The difference between the CP-odd and C.P-even cases is slight in the angular distribution, the ('P-odd distribution is enhanced relative to the CP-even case for the backward region of the angular phase space, ie. $\cos \theta_{\tau}>0$. The effect is small however and, given that it occurs where the differential cross-section is smallest, it is unlikely that it could be experimentally resolved. The invariant mass distributions might be more useful; the non-standard CPV case has a somewhat broader plateau and pupulates phase space more evenly than that of the CPeven case. However, because the differences are small, it is doubtful that these distributions could, once a non-standard cross-section indicated an anomalous coupling, distinguish the two possibilities of CP-even or CP-odd. In the following sectiın, we explore less ambiguous indicators of CP violation.

\subsection{Asymmetries as Indicators of CP Violation}

A "cleaner", and more intuitive, means of identifying a CP-violating contribution is to directly . search for evidence of the breaking of the symmetry. Even a small amount of CP violation in the triple boson vertex could in principle produce clear experimental signatures. These signatures would consist of asymmetries; for instance in the numbers of events between two CP-conjugate states. As an example, in the process $e^{+} e^{-} \rightarrow W^{+} W^{-}$, a difference between the numbers of $W^{+}$ bosons emitted in the "forward" direction, and the number of $W$ - bosons in the "backward" direction, as it distinguishes between these CP-conjugate states, would indicate the breaking of CP. Different types of observable asymmetries have been suggested as possible indicators of CPV [75], these include width asymmetries,

$$
A=\frac{\Gamma(i \rightarrow f)-\Gamma(\tilde{i} \rightarrow \tilde{f})}{\Gamma(i \rightarrow f)+\Gamma(\tilde{i} \rightarrow \tilde{f})}
$$

(where $\tilde{i}$ and $\tilde{f}$ are the CP-conjugate initial and final states), partial rate asymmetries such as energy and angular asymmetries,

$$
A=\frac{\sum_{f \in S} d \sigma(i \rightarrow f)-\sum_{f e \dot{S}} d \sigma(i \rightarrow f)}{\sum_{f \in S} d \sigma(i \rightarrow f)+\sum_{f \in \dot{S}} d \sigma(i \rightarrow f)}
$$


(where $S$ and $\tilde{S}$ are $C P$-conjugate kinematic ranges), and ('P-odd correlations

$$
<\Theta>=\frac{\int d \sigma(\Theta) \Theta(\vec{p}, \vec{s})}{\int d \sigma(\Theta)}
$$

where $\Theta(\vec{p}, \vec{s})$ is a CP-odd operator, ie. it changes sign under the (.P operation).

In our analysis we follow the last two approaches. We search for non-zero asymmetries ant expectation values in certain measurable observables as evidence for a ( $P V$ ('ontribution to the' TBV. The observables we consider are defined in terms of the final state lepton and anti-lepton momenta, and or, polar and azimuthal angles and thus avoid any ambiguity from neutrino nondetection.

We define the polar and azimuthal angles of the final state lepton and anti-lepton $(\theta, \phi)$ and $(\bar{\theta}, \bar{\phi})$ through the momentum parametrization,

$$
\begin{aligned}
& \vec{p}=|\vec{p}|(\sin \phi \sin \theta, \sin \phi \cos \theta, \cos \phi) \\
& \vec{q}=|\vec{q}|(\sin \bar{\phi} \sin \bar{\theta}, \sin \bar{\phi} \cos \bar{\theta}, \cos \bar{\phi})
\end{aligned}
$$

where $\vec{p}$ and $\vec{q}$ are the three-momenta of the outgoing lepton and antilepton, respertively. Sillce the initial state of $e^{+}$and $e^{-}$is CP invariant, the effect of the CP transformation is to reverss. all the momenta of final state particles and to interchange particles with antiparticles. With our parametrization of the angles, a flip in momenta is equivalent to $(\theta, \phi) \rightarrow(\pi+\theta, \pi-\phi)$. With the additional switch of particle and antiparticle, the CP operation is equivalent to the following transformation amongst the angular variables:

$$
(\theta, \phi, \bar{\theta}, \bar{\phi}) \stackrel{C P}{\leftrightarrow}(\pi+\bar{\theta}, \pi-\bar{\phi}, \pi+\theta, \pi-\phi)
$$

If the transition matrix $M$ is hermitian, then $C P \bar{T}$ invariance gives the following relation for $\mathbf{M}$

$$
M_{\sigma \bar{\sigma}, \lambda \bar{\lambda}}=M_{-\bar{\sigma},-\sigma,-\bar{\lambda},-\lambda}^{*}
$$

which is identical to the corresponding expression for $C P$ invariance but for the additional complex conjugation. The combined $C P \bar{T}$ is therefore equivalent to the following transformation amongst the angular variables

$$
(\theta, \phi, \bar{\theta}, \bar{\phi}) \stackrel{C}{\leftrightarrow} \dot{T}(\pi-\bar{\theta}, \pi-\bar{\phi}, \pi-\theta, \pi-\phi)
$$


We can now classify angular distributions according to their behaviour under C.P and CP $\tilde{T}$, any distribution that changes sign under the CP transformation is called CP-odd etc. $A$ violation of the (.P $\tilde{T}$ symmetry reflects the non-hermiticity of the transition matrix; the hallmark of final state interactions. To investigate the possibility of CPV in the gauge boson sector, whilst neglecting such final state interactions, we should concentrate on CP-odd and CP $\tilde{T}$-even variables.

The expectation value of any CP-odd variable, if CP is a good symmetry, must vanish. Accordingly, a non-zero expectation value for, or an asymmetry in the distribution of, a CPodd variable can be interpreted as evidence of $\mathrm{CP}$ violation in the underlying dynamics. In the following sections, we explore the expected magnitudes of such asymmetries and expectation values for non-zero values for the CPV couplings $\tilde{\kappa}_{V}$ and $\tilde{\lambda}_{V}$. For the sake of simplicity, we keep all other coupling parameters equal to their Standard Model values, eg. $\kappa_{\gamma}=\kappa_{Z}=1.0$, except to compare the cases of CP-even and CP-odd non-standard couplings. Since the imaginary parts of $\tilde{\kappa}_{V}$ and $\tilde{\lambda}_{V}$ break the CP $\tilde{T}$ symmetry, we restrict ourselves to the real parts of the coup.ings.

\subsubsection{CP-odd variables}

Hagiwara $e t$ al. [20] examined the inclusive angular distributions for the leptonic decay products of $e^{+} e^{-} \rightarrow W^{+} W^{-}$and classified them according to their symmetry properties under CP and C.P $\tilde{T}$ as even-even,even-odd,odd-even, and odd-cdd. They reported that the most sensitive oddeven angular variable was proportional to $S$, defined as:

$$
S=\sin \phi \sin \theta+\sin \bar{\phi} \sin \bar{\theta}
$$

It is a simple matter to verify that $S$ has the necessary symmetry properties.

$$
\begin{gathered}
S \stackrel{C P}{\rightarrow}-S \\
S \stackrel{C P \dot{T}}{\rightarrow} S
\end{gathered}
$$

It is possible to understand somewhat why $S$ should show greater sensitivity to non-zero CPV form factors compared to other odd-even distributions, eg. $\sin \theta \cos ^{2} \phi$. With only real pieces, the CPV form factors $\tilde{\kappa}_{V}, \tilde{\lambda}_{V}$ contribute imaginary parts to the ainplitude because of the phese difference in the effective vertex between the CP-odd terms and the CP-even terms. It has been shown previously that obearvation of $C P$ violation requires a source of complex phase in addition 
to the one due to the explicit C.PV form factors. The complex dependence of the amplitudes on the azimuthal angles $\phi, \bar{\phi}$ provides automatically the additional complex phase on requires. $A$ large sensitivity can therefore be easiest obtained by examining distributions involving sines of azimuthal angles, where the imaginary part from $C P$ violation interferes with phase differences amongst the helicity amplitudes.

Motivated by the analysis of Chang et al. [37], we also define a CP-odd, ('P'T-even variable $Q$ in terms of the momenta of the outgoing lepton and antilepton as follows:

$$
Q=\overrightarrow{k_{2}} \cdot\left(\overrightarrow{p_{1}} \times q_{1}\right)
$$

where $\overrightarrow{p_{1}}$ is the vector momenta of the outgoing lepton, and $\overrightarrow{q_{i}}$ that of the antilepton. The required symmetry properties under $\mathrm{CP}$ and $\mathrm{CP} \tilde{T}$ can be easily verified.

If $C P$ is a valid symmetry, then a differential cross-section in a C.P-odd variable such as $S$ (or $Q)$ would necessarily be symmetric about $S=0(Q=0)$.

$$
\frac{d \sigma}{d S} q P \frac{d \sigma}{d(-S)}
$$

The presence of CP violating couplings such as $\tilde{\kappa}_{V}, \tilde{\lambda}_{V}$ will manifest themselves in the loss of this equality. We can more rigorously define such an asymmetry by counting events where $S<0$, S $>0$ and evaluating the asymmetry

$$
A_{C P}^{S}=\frac{N(S>0)-N(S<0)}{N(S>0)+N(S<0)}
$$

A non-zero value for $A_{C}^{S}$ thus indicates the loss of the CP symmetry. This asymmetry in the $\mathrm{S}$ distribution should therefore be zero, both for the Standard Model and also for non-standari but CP-respecting triple vertex contributions, such as the couplings $\mathrm{kV}$.

In practice, in calculating the asymmetry as defined above, the limitations of Monte-Carlo round-off errors and finite distribution bin size prevent a precinion analysis. We instead calculate the asymmetry as follows. For every final state configuration of lepton and antilepton, we calculate an event weight for the given momenta configuration, as well as an event weight for the CP-conjugate final state configuration (particle interchanged with antiparticle, and momenta reversed). The difference between these two weights is therefore a measure of CP invariance [76]. 
An equivalent expression for $A_{r \cdot p}^{S}$ is then

$$
A_{C P}^{S}=\frac{\int \frac{d \sigma}{d|S|} d|S|-\int \frac{d \sigma^{C P}}{d|S|} d|S|}{\int \frac{d \sigma}{d|S|} d|S|+\int \frac{d \sigma^{C P}}{d|S|} d|S|}
$$

where $\sigma$ and $\sigma^{C P}$ correspond to the two different $C P$ conjugate events weights.

This formalism also allows us to easily determine the corrresponding asymmetries in the individual helicity amplitudes. The helicity asymmetries are defined exactly analagous to that in the total cross-section. As a check, we verified thai the component helicity asymmetries, when weighted by the relative contribution of their corresponding amplitude to the total cross-section, should combine to give the same total asymmetry as obtained from the total cross-section. For instance, the $\mu^{+} \tau^{-}$process has two contributing helicity amplitudes, $(+-+-)$and $(-++-)$. Defining $A_{+-+-}^{C P}$ and $A_{-++-}^{C P}$ just as we did previously for the total cross-section, we calculate

$$
A_{C P}^{\text {hel }}=\frac{\left(A_{ \pm-+-}^{C P}\right) *\left(\sigma_{+-+-}\right)+\left(A_{-++--}^{C P}\right) *\left(\sigma_{-++-}\right)}{2 \sigma_{\text {total }}}
$$

and require that $A_{C P}^{S}=A_{C P}^{\text {hel }}$. The factor of two takes into account that our distribution is in $|S|$, not $\mathbf{S}$. The form of this expression suggests that partial cancellation between helicity amplitudes might arise; two large asymmetries of opposite sign conbining to give a smaller net asymmetry in the total cross-section.

If, instead of binning in either of the two CP-conjugate weights, we bin in the difference between them, we can define a "differential asymmetry" variable $\chi(S)$ as

$$
X(S)=\frac{\left(\frac{d \sigma}{d(S)}-\frac{d \sigma}{d(-s)}\right)}{\sigma}
$$

and as a further check on the Monte Carlo algorithm, verified that

$$
A_{S}^{k}=\int x(S) d S
$$

returned the same value as $A_{C P}^{S}$. 


\subsubsection{Asymmetries in $S$ and $Q$}

For $\tilde{x}_{V}$ and $\tilde{\lambda}_{V}$ equal to 1 , we show in Table $\bar{t} .5$ the asymmet ries $A_{i}, p$ in the tot al arosi-sintion for the lepton channels $\mu^{+} r^{-}, \mu^{+} \mu^{-}$, and $e^{+} \rho^{-}$. (The $\mu^{+} c^{-}$channel. without, its charge eonjukate process $\mu^{-} e^{+}$, is not CP invariant, and so cannot be used to search for ('P). With our furmalisin of defining the asymmetries through the difference betwen event weights, the atsymumetru's in the total cross-section for the variable $A_{C P}^{Q}$ are the sane as $\mathcal{A}_{f}^{S} p$. The differences hetwern $S$ anml $Q$ will manifest themselves in the differential asymmetries. 'The ('P-odd couplings coutrihute' to first-order quadratically (for the most part); there is thus little difference in ibymum rios between negative and positive $\tilde{\kappa}_{V}$ and $\bar{\lambda}_{V}$. We therefore restrict out study to positive anomalous couplings $\tilde{\kappa}_{V}, \tilde{\lambda}_{V}=1.0$. For teference, when the couplings of the TBV were set at. their SM values, as well as for non-standard but CP-even coupleng deviations, the asymmetries were of the order $10^{-14}-10^{-18}$.

Table 7.5: Asymmetries $A_{C P}^{S}\left(\times 10^{3}\right)$ in Total ( Pross-Section

\begin{tabular}{|l|l|l|l|l|l|}
\hline & & $\bar{\kappa}_{\gamma}=1$ & $\bar{\kappa}_{Z}=1$ & $\bar{\lambda}_{\gamma}=1$ & $\bar{\lambda}_{Z}=1$ \\
\hline$\mu^{+} \tau^{-}$ & $500 \mathrm{GeV}$ & -2.1 & -3.3 & -1.9 & -1.8 \\
\cline { 2 - 6 } & $1 \mathrm{TeV}$ & 0.58 & 1.1 & -1.9 & -2.0 \\
\hline \multirow{2}{*}{$\mu^{+} \mu^{-}$} & $500 \mathrm{GeV}$ & -1.3 & -0.67 & 3.2 & 3.9 \\
\cline { 2 - 6 } & $1 \mathrm{TeV}$ & -0.14 & -1.7 & -1.1 & -1.3 \\
\hline \multirow{2}{*}{$e^{+} e^{-}$} & $500 \mathrm{GeV}$ & -2.2 & -2.5 & -3.7 & -4.1 \\
\cline { 2 - 6 } & $1 \mathrm{TeV}$ & 0.16 & 0.85 & -6.3 & -6.7 \\
\hline
\end{tabular}

We notice that the asymmetries are of the same magnitude for each of the different. final state configurations, and that the higher energy does not guarantee larger asymmetries. 'The'

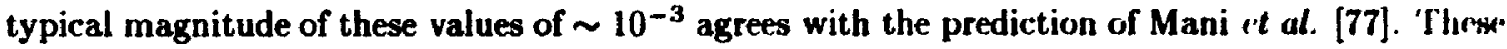
authors, instead of simple asymmetries, looked at expectation values of $(; \mathbf{P}$-odd variablers, and find $A \sim 10^{-3}$ for $\tilde{\kappa}$ or $\tilde{\lambda}=0.1$.

For an asymmetry in the total cross-section to be measurable, we require that the number of asymmetrical events $\Delta N$ exceed the fluctuations about the total number of events. 'I'hus our significance requirement for these asymmetries is, 


$$
\begin{aligned}
\Delta N & >\delta N \\
A \sigma L & >\sqrt{\sigma L} \\
A & >(\sigma L)^{-\frac{1}{2}}
\end{aligned}
$$

If we take a typical value for the cross-section of $\sigma \sim 0.1 \mathrm{pb}$, and with our assummed integrated luminosity $L=50 \mathrm{fb}-1$, this significance level is approximately $1.5 \%$. From this quick calculation, it seems that an asymmetry in the total cross-section will most likely be below the statistical significance level, and therefore unresolvable. The situation is even less encouraging if, instead of $\tilde{\kappa}_{V}=\tilde{\lambda}_{V}=1.0$, we explore the expected asymmetries for more realistic magnitudes for the anomalous couplings, ie. $\tilde{\kappa}_{V}$ and $\tilde{\lambda}_{V}$ at their detection limits.

Although it appears that any asymmetry in total cross-section will be below the level of statistical significance, asymmetries in the helicity amplitudes that contribute to the total crosssection might be detectable. Returning to Eq. (7.11), we see that an asymmetry in the total crosssection is a result of a combination of asymmetries in the contributing helicity amplitudes, each weighted by their appropriate helicity cross-sections. Since the different helicity amplitudes have contributions from different TBV diagrams, with consequently different sensitivity to anomalous couplings, the asymmetries in these helicity amplitudes can differ in magnitude and sign. In principle therefore, large but opposing asymmetries can cancel each other to produce a smaller resultant asymmetry in the total cross-section. We demonstrate this idea by examining the asymmetries in the CP-odd variable $Q$, both total and helicity, for the $\mu^{+} \mu^{-}$channel at $\sqrt{s}=1$ TeV.

$$
\begin{aligned}
& \text { Total } A=2.8 \times 10^{-4} \\
& (+-+) \quad A=1.4 \times 10^{-3} \\
& (-++-) \quad A=2.0 \times 10^{-4} \\
& (-+-+) \quad A=-2.1 \times 10^{-16} \\
& (+--+) \quad A=-8.1 \times 10^{-4}
\end{aligned}
$$

The very small asymmetry in the $(-+-+)$ amplitude reflects the fact that this amplitude has no dependence on the TBV; it is therefore unaffected by non-zero $\tilde{\kappa}_{V}$ and $\tilde{\lambda}_{V}$. The relatively 
large asymmetry in the $(+-+-)$ amplit ude is diminished by the oppositely signed asymmetry in (+ - + ) such that, in the total cross-section, the asymmetry is smaller than either of these "component" asymmetries. This effect can be more clearly demonstrated by considering the so-called "differential asymmetries" in the variable $x$, where $\downarrow$ is a function of either of the two CP-odd variables $S$ and $Q$. We show in Figure (7.3) the distributions in $X(S)$ for the $\mu^{+} T^{-}$ channel at $\sqrt{s}=500 \mathrm{GeV}$ for non-standard $\dot{\kappa}_{Z}=1.0$. We show also the approximate statistical error bounds about the SM expectation of $\chi(S)=0$, calculated from the differential cross-section $d \sigma / d S$

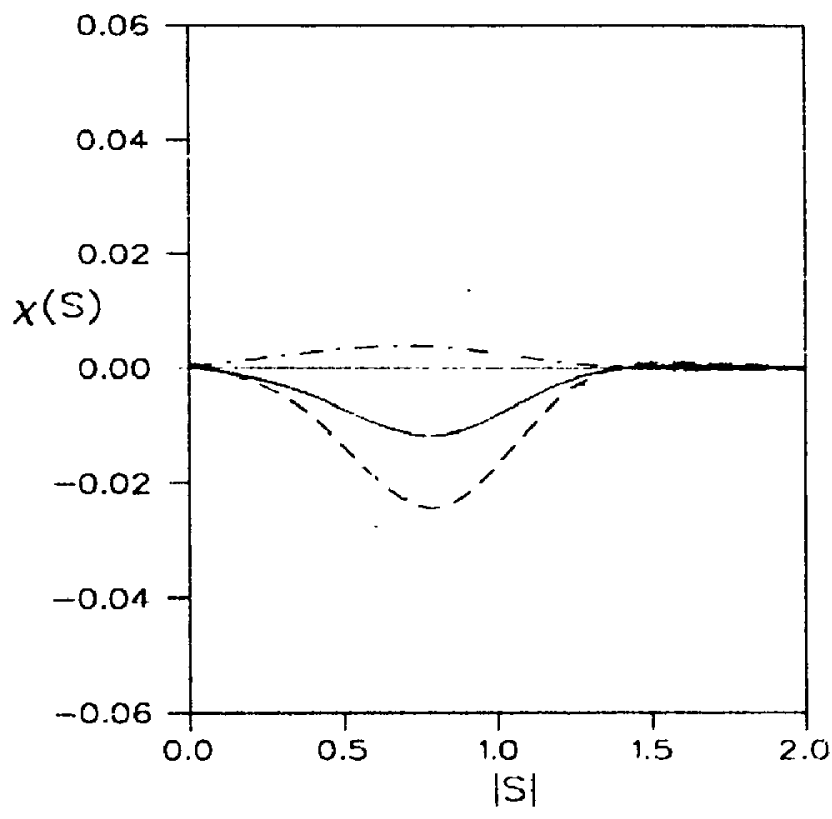

Figure 7.3: Distributions in $\chi(S)$ for the $\mu^{+} \tau^{-}$process at $\sqrt{s}=500 \mathrm{GeV}$ with $\tilde{\kappa}_{Z}=1.0$. The solid line corresponds to the total cross-section, the dot-dashed to the $(-++-)$ amplitude, and the dashed to the $(+-+-)$ amplitude. The asymmetries are divided by the appropriate cross-section, either total or component. The dotted line represents the statistical error bounds.

We see that while the asymmetry in the total cross-section, as it is bousded by the error bars, would be below the statistical significance level and so unresolvable; that in $\therefore i t(+-+-)$ helicity amplitude might be measurable. A polarized beam facility, by separately measuring the different helicity components, might be able to measure these helicity asymmetries.

The $e^{+} e^{-}$channel, with all 6 possible helicity amplitudes contributing to the total crostssection, demonstrates another interesting helicity eftect in the CP asymmetries. The two TBV 
dependent diagrams with a neutrai gauge boson and a $W$ in the t-channel, Figures (3.3a) and (3.3b), contribute to the $(++++)$ and $(----)$ a!nplitudes, respectively. Because these two diagrams are CP-conjugate, these helicity amplitudes, each containing the contribution of one or the other, separately violate the CP-symmetry even for standard model values for the couplings $\tilde{\kappa}_{V}$ and $\bar{\lambda}_{V}$. The total cross-section. with a contribution from each of the two separately CP violating helicity amplitudes, will however respect the CP symmetry. We demonstrate this phenomena by looking at the distribution in $x(Q)$ for the $e^{+} e^{-}$process at $\sqrt{s}=1 \mathrm{TeV}$. We have normalized $|Q|$ such that it ranges $0 \leftrightarrow 1$.

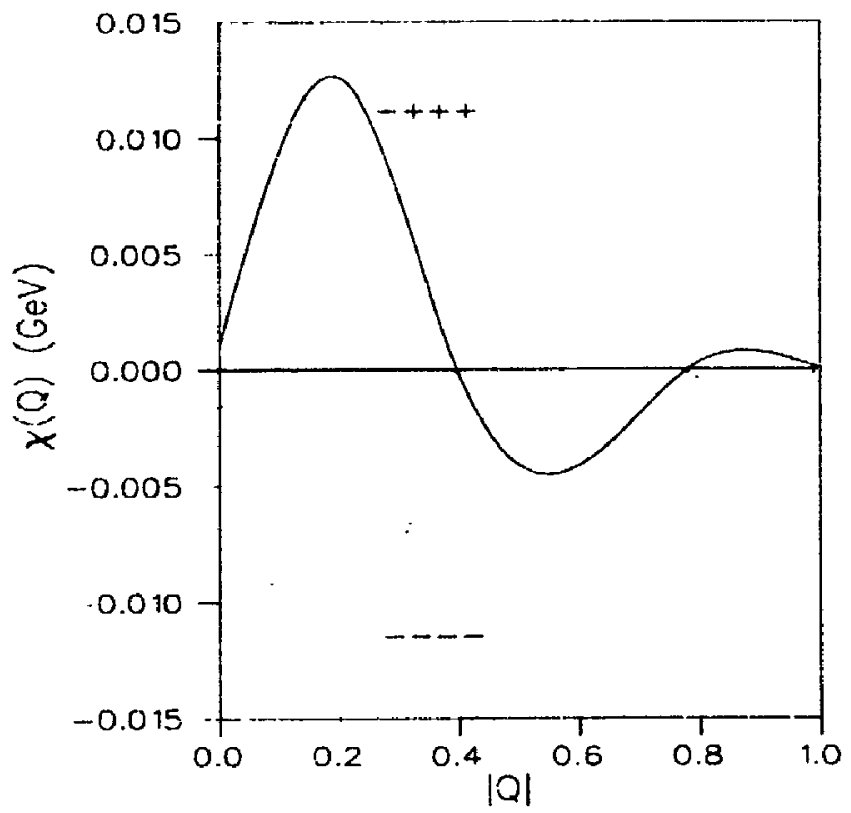

Figure 7.4: Distributions in $\chi(Q)$ for the $e^{+} e^{-}$process at $\sqrt{8}=1000 \mathrm{GeV}$ with SM couplings. The solid line corresponds to the $(++++)$ helicity amplitude and the dotted line to the (-- - ) amplitude. The asymmetry in the total cross-section and the other four helicity amplitudes are too small to be visible on this scale.

Although the differential asymmetries in the two helicity amplitudes $\chi(Q)_{(++++)}$as:d $\chi(Q)_{(----1}$ are both non-zero ; their stim respects CP invariance, as indicated by the visible cancellation that occurs between the two curves.

The nature of the curves of Figure (7.4) suggests an alternative method of resolving the CP asymmetries. The distributions in $\chi(S)$ or $\chi(Q)$, in either the total or component cross-sections, can in general have both "positive" and "negative" sectors. By summing over both types of 
regions, the net asymmetry is smaller than if the phase space was restricted to cither one or the other. By cutting on the CP-odd variables $S$ or $Q$ to restrict phase space to one or the other type of region, the net asymmetry can be maximized. For instance, restrieting $|Q|<0.5$ for the $(++++)$ helicity amplitude of Figure (7.4) excludes the negative asynumetry contribution; the net asymmetry is then larger. Since the differential cross-section $d \sigma / d Q$ is strongly peaked about $Q=0$; such a cut that excludes the relatively small contribution of the "tail" regions does not then significantly reduce the expected number of events. We thereby maximize asymmetries without significantly diminishing the cross-section and statistics. 


\section{Appendix A}

\section{Feynman Rules}

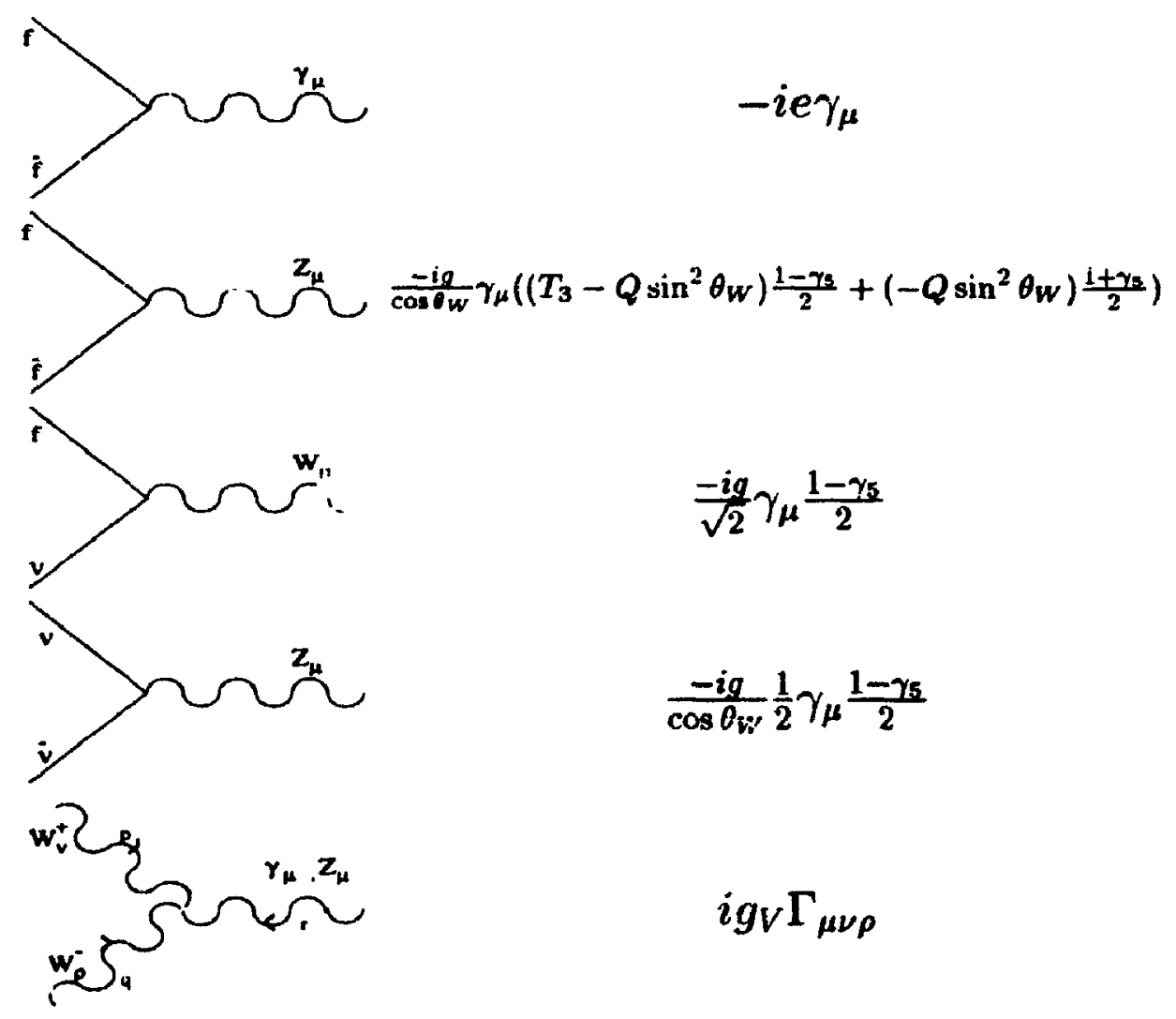




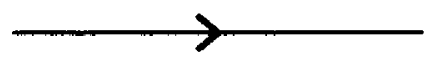

$\frac{i}{\not-m}$

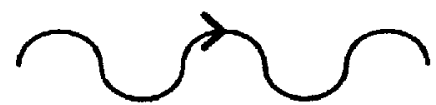

$\frac{i\left(-g^{\mu \nu}+k^{\mu} k^{\nu} / M_{V}^{*}\right)}{k^{2}-M_{V}^{2}}$ 


\section{Appendix B}

\section{Full Set of Contributing Diagrams}

This Appendix contains the full set of contributing Feynman diagrams, grouped according to the different types of the four lepton production processes. Figure (B.1) contains the 9 diagrams that contribute to the $\mu^{+} \boldsymbol{r}^{-}$channel. The $\mu^{+} e^{-}$channel includes contributions from the diagrams of Figure (B.1) as well as the 9 diagrams of Figure (B.2) for a total of 18 . The $\mu^{+} \mu^{+}$channel has contributions from the 19 diagrams of Figure (B.3), as well as from the same 9 diagrams of Figure (B.1) that contributed to both the $\mu^{+} \tau^{-}$and $\mu^{+} e^{-}$processes, for a total of 28 . The $e^{+} e^{-}$ channel has contributions from the 9 diagrams of Figure (B.1), the 9 of Figure (B.2), the 19 of Figure (B.3), and from an additional 19 diagrams of Figure (B.4).

In each diagram, the upper initial state fermion line corresponds to the positron, the lower initial state fermion line to the electron.

The labelling convention for the diagrams matches that of Appendix D where the expressions for the diagrams' amplitudes are given. 

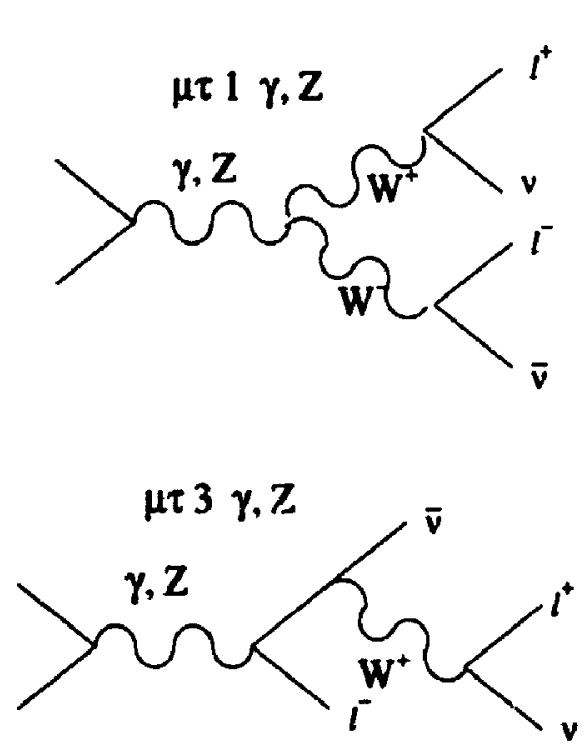

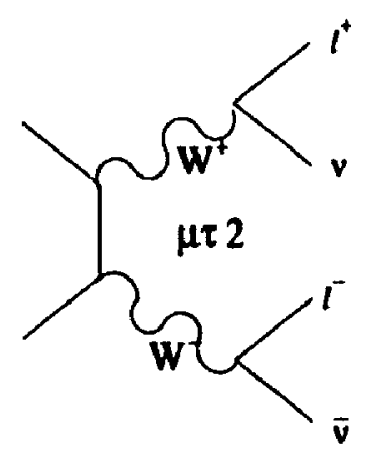

$\mu \tau 4 \gamma, 2$
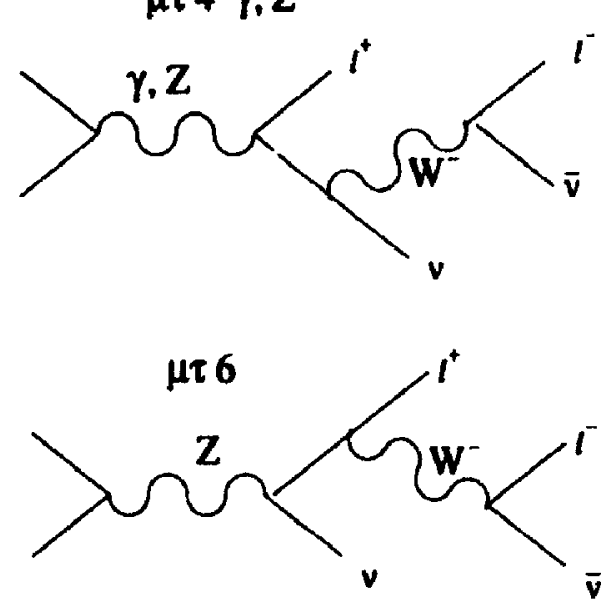

Figure B.1: 9 diagrame that contribute to the $\mu^{+} \tau^{-}$final state. 

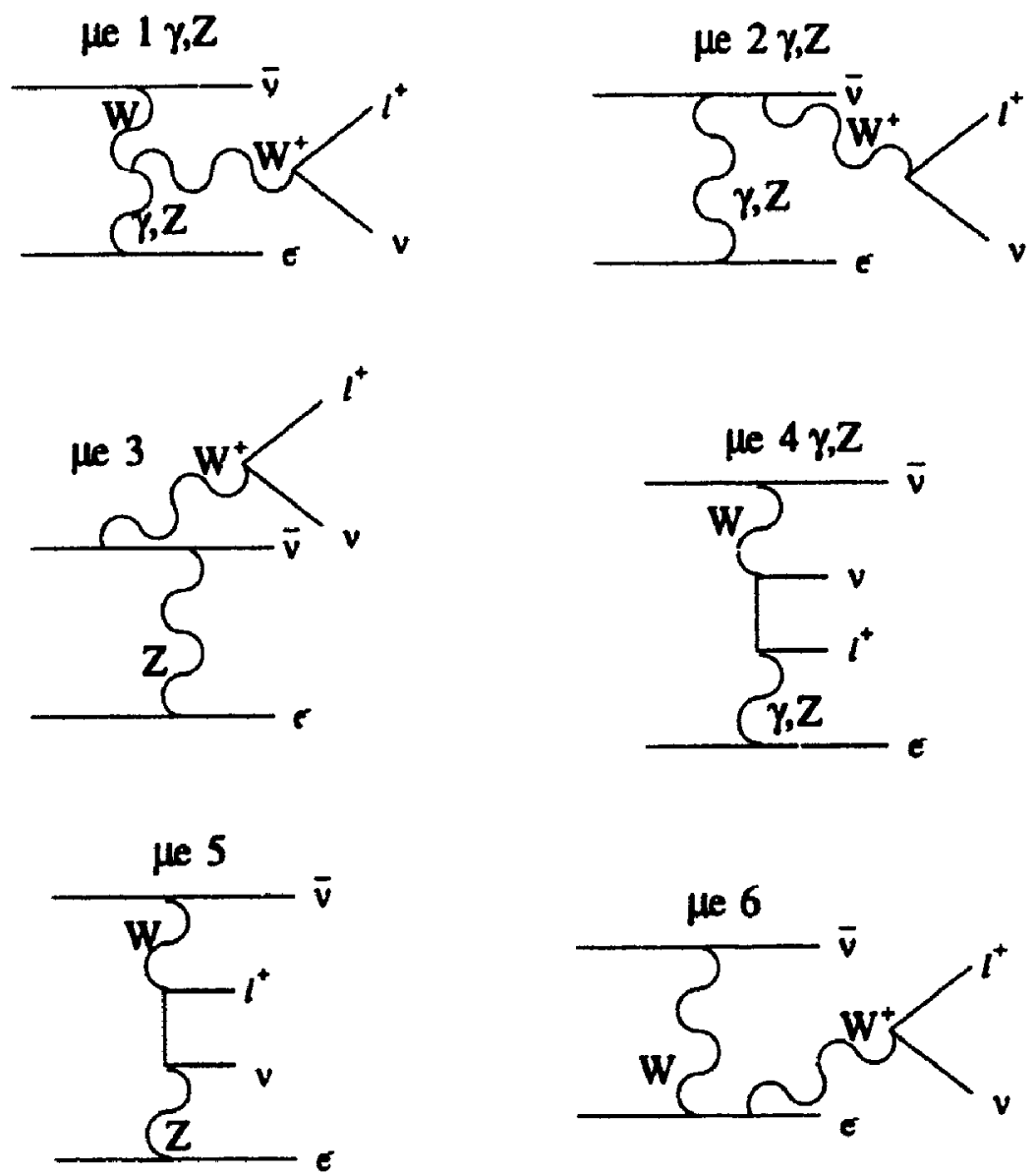

Figure B.2: 9 diagrams extra to those of the $\mu^{+} \tau^{-}$channel that contribute to the $\mu^{+} e^{-}$final state 

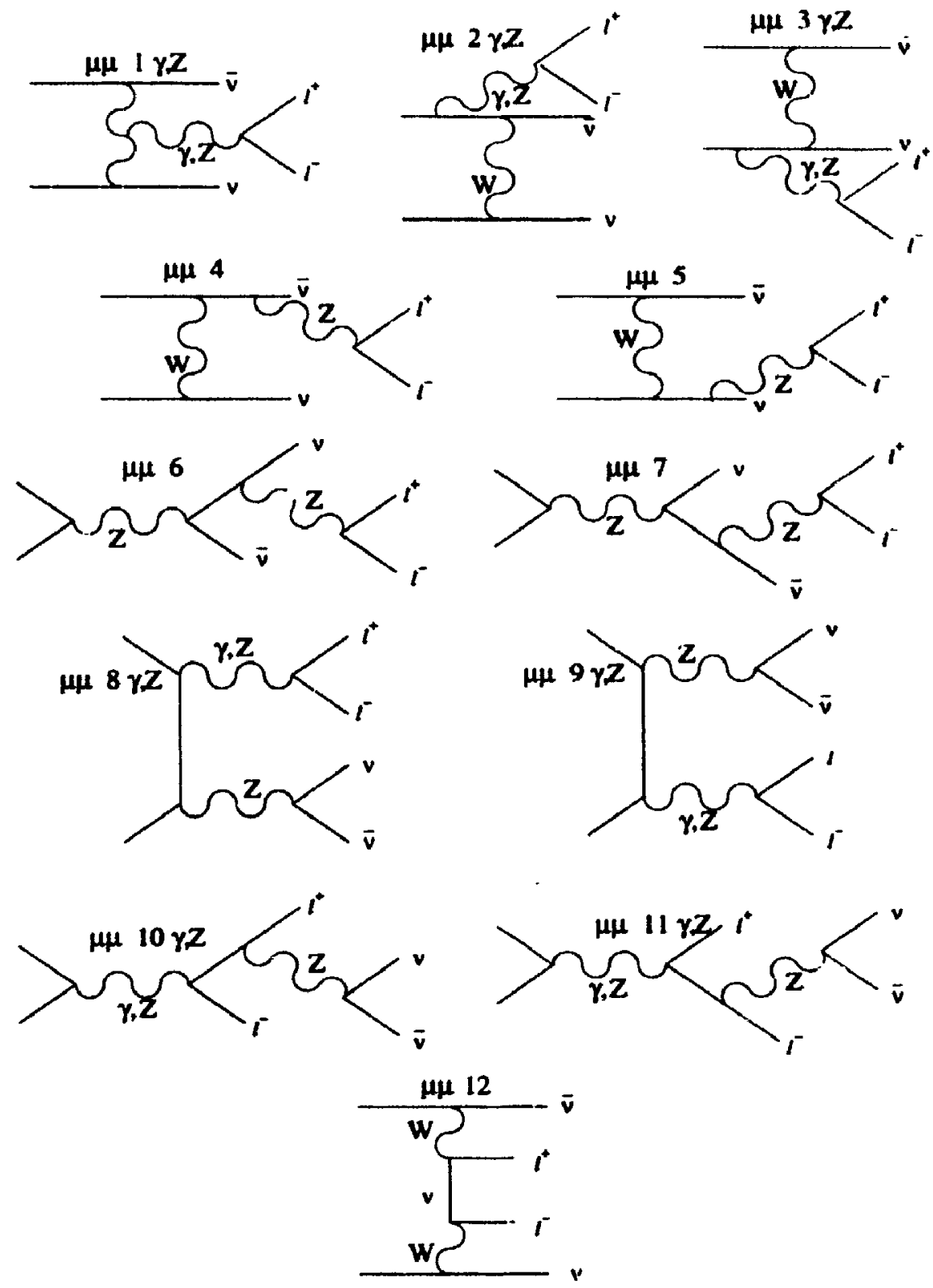

Figure B.3: 19 diagrams extra to those of the $\mu^{+} \tau-$ channel that contribute to the $\mu^{+} \mu^{-}$final state 

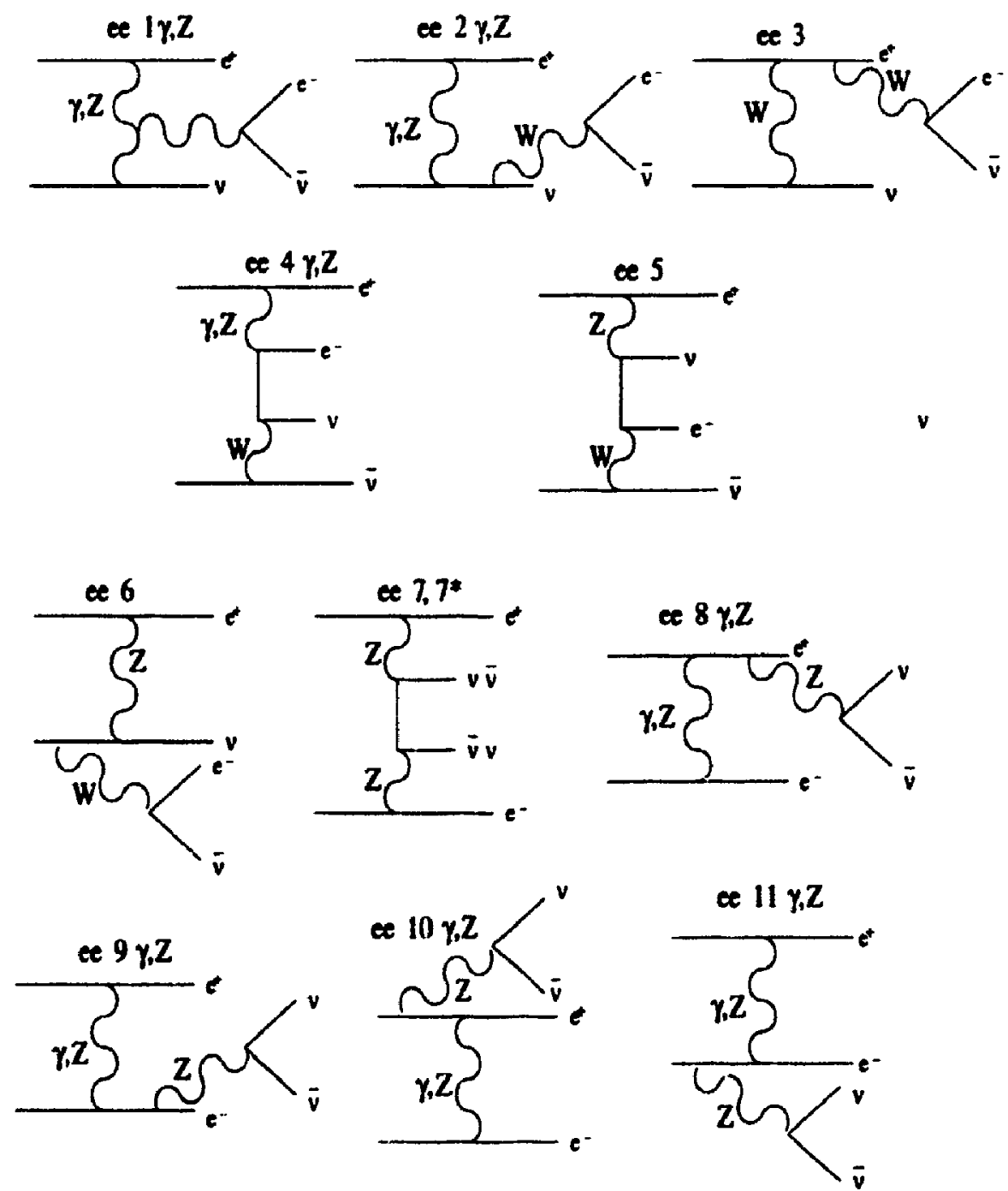

Figure B.4: 19 diagrams extra to those of the $\mu^{+} \tau^{-}, \mu^{+} e^{-}$, and $\mu^{+} \mu^{-}$channels that contribute to the $e^{+} e^{-}$final state 


\section{Appendix C}

\section{CALKUL Formalism}

The evaluation of higher order tree graphs through the standard algebraic techniquess beromess impractical when bcth the number of external lines and the number of diagrams involved are . large. The complexity is due to the fact that, in these standard terhniques, we must evaluate $|M|^{2}$, through determination of traces over strings of Dirac matrices. Because we evaluate $|M|^{2}$. the number and lengths of these traces increases very rapidly with the order of prerturbation theory and the number of contributing diagrams. Any technique which, instead of $|M|^{2}$, evaluaters $\mathrm{M}$, will be much less cumbersome, both analytically and computationally. The ('Al,KUI, [5K] formalism is such a method.

The technique consists of analytically reducing the strings of spinors and gamma matricess in the expressions of the amplitudes to products of sandwiches of spinors like $\bar{u}\left(p_{1}\right) u\left(p_{2}\right)$, threw: spinor sandwiches are then easily computed.

We introduce two four-vectors, $k_{0}^{\mu}$ and $k_{1}^{\mu}$, which satisfy the following relations:

$$
\begin{gathered}
k_{0} \cdot k_{0}=0 \\
k_{1} \cdot k_{1}=-1 \\
k_{0} \cdot k_{1}=0
\end{gathered}
$$

and also introduce the basic spinors

$$
u_{-}\left(k_{0}\right) \bar{u}_{-}\left(k_{0}\right)=w_{-} k_{u}
$$

where $\omega_{-}$is the projection operator 


$$
\omega_{ \pm}=\frac{1}{2}\left(1 \pm \gamma_{5}\right)
$$

and

$$
u_{+}\left(k_{0}\right)=k_{1} u_{-}\left(k_{0}\right)
$$

These two basic spinors, $u_{-}\left(k_{0}\right)$ and $u_{+}\left(k_{0}\right)$, allow us to construct spinors for any other lightlike momentum $\boldsymbol{\eta}$ as follows:

$$
u_{\lambda}(p)=\frac{p u_{-\lambda}\left(k_{0}\right)}{\sqrt{2 p \cdot k_{0}}}
$$

Two identities are essential for the reduction of the trace strings into spinor sandwiches; these are the spin sum, which in the massless limit becomes:

$$
\sum_{\lambda} u_{\lambda}(p) \bar{u}_{\lambda}(p)=p
$$

and the Chisholm identity:

$$
\bar{u}_{\lambda}\left(p_{1}\right) \gamma^{\mu} u_{\lambda}\left(p_{2}\right) \gamma_{\mu}=2 u_{\lambda}\left(p_{2}\right) \bar{u}_{\lambda}\left(p_{1}\right)+2 u_{-\lambda}\left(p_{1}\right) \bar{u}_{\lambda}\left(p_{2}\right)
$$

where $\lambda= \pm 1$ for the two different helicities. Only two of the four possible sandwiches are non-zero, we define them as follows:

$$
s\left(p_{1}, p_{2}\right)=\bar{u}_{+}\left(p_{1}\right) u_{-}\left(p_{2}\right)=-s\left(p_{2}, p_{1}\right)
$$

and

$$
t\left(p_{1}, p_{2}\right)=\bar{u}_{-}\left(p_{1}\right) u_{+}\left(p_{2}\right)=s\left(p_{2}, p_{1}\right)^{*}
$$

Once the amplitude has been expressed in terms of $s\left(p_{i}, p_{j}\right)$ and $t\left(p_{i}, p_{j}\right)$, it can be readily computed. By choosing $k_{0}$ and $k_{1}$ appropriately, the forms of $s$ and $t$ can be greatly simplified, maxing for easy programming. With the following choices for $k_{0}$ and $k_{1}$ :

$$
\begin{aligned}
& k_{0}^{\mu}=(1,1,0,0) \\
& k_{1}^{\mu}=(0,0,1,0)
\end{aligned}
$$


we obtain

$$
s\left(p_{1}, p_{2}\right)=\left(p_{1}^{y}+i p_{1}^{z}\right) \frac{\sqrt{p_{2}^{0}-p_{2}^{x}}}{\sqrt{p_{1}^{0}-p_{1}^{x}}}-\left(p_{2}^{y}+i p_{2}^{*}\right) \frac{\sqrt{p_{1}^{0}-p_{1}^{x}}}{\sqrt{p_{2}^{0}-p_{2}^{x}}}
$$

We illustrate the technique by evaluating the amplitude for the t-r haund neut rimo diagram that contributes to each of the final state contigurations. Our momenta are defincel as follows:

$$
e^{+}\left(l_{i_{1}}\right)+e^{-}\left(k_{2}\right) \rightarrow l^{-}\left(p_{1}\right) \bar{\nu}\left(p_{2}\right) l^{+}\left(q_{1}\right) \nu\left(y_{2}\right)
$$

The amplitude is then (in the massless fermion approximation)

$$
M=\frac{-i g^{4}}{64} \frac{\bar{u}\left(k_{1}\right) \gamma^{\mu}\left(1-\gamma_{5}\right)\left(k_{2}-\not p\right) \gamma^{\nu}\left(1-\gamma_{5}\right) u\left(k_{2}\right) \bar{u}\left(q_{2}\right) \gamma_{\mu}\left(1-\gamma_{5}\right) u\left(\psi_{1}\right) \bar{u}\left(p_{1}\right) \gamma_{\nu}\left(1-\gamma_{5}\right) u\left(p_{2}\right)}{\left(k_{2}-p\right)^{2}\left(q^{2}-M_{\bar{W}^{\mu}}^{2}\right)\left(p^{2}-M_{\bar{w}}^{2}\right)}
$$

Using the projection operators, and rearranging, we get

$$
M=\frac{-i g^{4}}{4} \frac{\bar{u}\left(k_{1}\right)\left(\bar{u}\left(q_{2}\right) \gamma_{\mu} u_{-}\left(q_{1}\right) \gamma^{\mu}\right)\left(k_{2}-p\right)\left(\bar{u}\left(p_{1}\right) \gamma_{\nu} u_{-}\left(p_{2}\right) \gamma^{\nu}\right) u_{-}\left(k_{2}\right)}{\left(k_{2}-p\right)^{2}\left(q^{2}-M_{W}^{2}\right)\left(p^{2}-M_{W}^{2}\right)}
$$

we use the spin summation identity to express $\left(k_{2}-\not p\right)$ as a sum of spinor products, and then the Chisholm identity to reduce the above to:

$$
M=-\frac{i g^{4}}{4} \frac{\bar{u}_{-}\left(k_{1}\right) u_{+}\left(q_{2}\right) \bar{u}_{+}\left(q_{1}\right)\left(u_{-}\left(k_{2}\right) \bar{u}_{-}\left(k_{2}\right)-u_{-}\left(p_{2}\right) \bar{u}_{-}\left(p_{2}\right)\right) u_{+}\left(p_{1}\right) \bar{u}_{+}\left(p_{2}\right) u_{-}\left(k_{2}\right)}{\left(k_{2}-p\right)^{2}\left(q^{2}-M_{W}^{2}\right)\left(p^{2}-M_{W}^{2}\right)}
$$

which, using the definition of $s$ and $t$, we write as:

$$
M=-\frac{i g^{4}}{4} \frac{t\left(k_{1}, q_{2}\right)\left(s\left(q_{1}, k_{2}\right) t\left(k_{2}, p_{1}\right)-s\left(q_{1}, p_{2}\right) t\left(p_{2}, p_{1}\right)\right) s\left(p_{2}, k_{2}\right)}{\left(k_{2}-p\right)^{2}\left(q^{2}-M_{W}^{2}\right)\left(p^{2}-M_{W}^{2}\right)}
$$

We then evaluate the amplitude numerically as a complex number, and squaring the sum of each contributing diagrams amplitude, we obtain the complete $|M|^{2}$. The diagram chowen ass the example, because it involved only $W$-couplings, contributes only to the (+- +-) anplitude. More generally, a diagram will contribute to more than one helicity amplitude. For instancen, the s-channel $\mathbf{W}$-pair production diagrams contribute to $(+-+-)$ as well as tos $(-++-)$. Thr. amplitude for this diagram can therefore be written as

$$
M=M_{(+-+-)}+M_{(-++-)}
$$

and each piece is added to the corresponding rontributions from other diagrams, thesse wurns ar. 
then squared. The total cross section can then be schematically written as:

$$
\sigma_{\text {total }}=\int\left(M_{(+-+-)}^{2}+M_{(-++-)}^{2}\right) d(L I P S)
$$

We see that the CALKUL formalism retains the helicity information in an easily accessible form. 


\section{Appendix D}

\section{Amplitude Expressions}

The notation for labelling the diagrams' amplitudes is as defined in Appendix B, where the corresponding Feynman diagrams are listed. The diagrams are classified as to which type of the four lepton processes they contribute to, thus we have classes of diagrams labelled as $\mu \tau, \mu e, \mu \mu$, and ee. The momenta are defined as follows

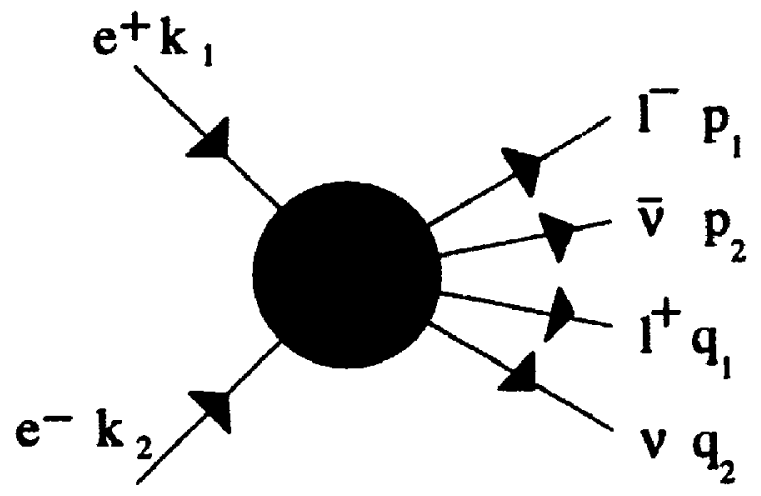

and $k=k_{1}+k_{2}, p=p_{1}+p_{2}, q=q_{1}+q_{2}$, and $k=q+k$. As a shorthand notation in the numerators of the amplitude expressions, we label the momenta $k_{1}, k_{2}, p_{1}, p_{2}, q_{1}$, and $q_{2}$ by the integers 1 through 6 , respectively. Thus $s(3,5)$ corresponds to the spinor product $s\left(p_{1}, q_{1}\right)$ etc. We define the Z-fermion coupling factors as

$$
A_{Z}=4 \sin ^{2} \theta_{w}-2 \text { and } B_{Z}=4 \sin ^{2} \theta_{w}
$$




$$
\begin{aligned}
M_{\mu+1 \gamma} & =\frac{i g^{4} \sin ^{2} \theta_{W}\left[T_{+-+-}^{\mu \tau 1}+T_{-++-}^{\mu \tau 1}\right]}{s\left(p^{2}-M_{W}^{2}-i \Gamma_{W} M_{W}\right)\left(q^{2}-M_{W}^{2}-i \Gamma_{W} M_{W}\right)} \\
M_{\mu \tau 1 Z} & =\frac{-i g^{4}\left[A_{Z} T_{+-+-}^{\mu+1}+B_{Z} T_{-++-}^{\mu+1}\right]}{4\left(s-M_{Z}^{2}\right)\left(p^{2}-M_{W}^{2}-i \Gamma_{W} M_{W}\right)\left(q^{2}-M_{W}^{2}-i \Gamma_{W} M_{W}\right)}
\end{aligned}
$$

$$
\begin{aligned}
T_{+-+-}^{\mu \tau 1}= & (\kappa v+1) t(1,3) s(4,2)(t(6,3) s(3,5)+t(6,4) s(4,5)) \\
& -2 t(3,6) s(5,4)(t(1,3) s(3,2)+t(1,4) s(4,2)) \\
& -(\kappa v+1) t(1,6) s(5,2)(t(3,5) s(5,4)+t(3,6) s(6,4)) \\
& +i \tilde{\kappa}_{v}(t(1,6) s(5,2)(t(3,1) s(1,4)+t(3,2) s(2,4)) \\
& -2 t(6,1) s(2,4)(t(3,1) s(1,5)+t(3,2) s(2,5)) \\
& +t(1,3) s(4,2)(t(6,1) s(1,5)+t(6,2) s(2,5))) \\
& +i \tilde{\lambda} v / 2 M{ }_{W}^{2}(2(t(3,1) s(1,4)+t(3,2) s(2,4))(t(6,1) s(1,3)+t(6,2) s(2,3)) t(3,1) s(2,5) \\
& +(t(6,1) s(1,4)+t(6,2) s(2,4)) t(4,1) s(2,5)) \\
& -2 p \cdot k(t(3,1) s(1,4)+t(3,2) s(2,4)) t(6,1) s(2,5)) \\
& -(t(3,1) s(1,4)+t(3,2) s(2,4))(t(6,1) s(1,5)+t(6,2) s(2,5)) \\
& (t(1,3) s(3,2)+t(1,4) s(4,2)) \\
& +p \cdot(p-k)(-4(t(3,1) s(1,5)+t(3,2) s(2,5)) t(6,1) s(2,4) \\
& +2(t(6,1) s(1,5)+t(6,2) s(2,5)) t(1,3) s(4,2)) \\
& +2(t(3,1) s(1,4)+t(3,2) s(2,4)) t(1,6) s(5,2)) \\
& +2(t(6,3) s(3,5)+t(6,4) s(4,5))(t(3,1)(s(1,3) t(3,1)+s(1,4) t(4,1)) s(2,4) \\
& +t(3,2)(s(2,3) t(3,1)+s(2,4) t(4,1)) s(2,4)) \\
& -2 p \cdot k(t(6,3) s(3,5)+t(6,4) s(4,5)) t(1,3) s(4,2) \\
& -(t(3,1) s(1,4)+t(3,2) s(2,4))(t(6,3) s(3,5)+t(6,4) s(4,5)) \\
& (t(1,3) s(3,2)+t(3,4) s(4,2))) \\
&
\end{aligned}
$$




$$
\begin{aligned}
& T_{-++-}^{\mu r 1}=\left(\kappa_{V}+1\right) s(1,4) t(3,2)(t(6,3) s(3,5)+t(6,4) s(4,5)) \\
& -2 t(3,6) s(5,4)(s(1,3) t(3,2)+s(1,4) t(4,2)) \\
& -\left(\kappa_{V}+1\right) s(1,5) t(6,2)(t(3,5) s(5,4)+t(3,6) s(6,4)) \\
& +i \bar{\kappa}_{V}(s(1,5) t(6,2)(t(3,1) s(1,4)+t(3,2) s(2,4)) \\
& -2 t(6,2) s(1,4)(t(3,1) s(1,5)+t(3,2) s(2,5)) \\
& +s(1,4) t(3,2)(t(6,1) s(1,5)+\ell(6,2) s(2,5))) \\
& +i \bar{\lambda}_{V} / 2 M_{W}^{2}(2(t(3,1) s(1,4)+t(3,2) s(2,4)) \\
& (t(6,1) s(1,3)+t(6,2) s(2,3)) t(3,2) s(1,5) \\
& +(t(6,1) s(1,4)+i(6,2) s(2,4)) t(4.2) s(1,5)) \\
& -2 p \cdot k(t(3,1) s(1,4)+t(3,2) s(2,4)) t(6,2) s(1,5)) \\
& -(t(3,1) s(1,4)+t(3,2) s(2,4))(t(6,1) s(1,5)+t(6,2) s(2,5)) \\
& (s(1,3) t(3,2)+s(1,4) t(4,2)) \\
& +p \cdot(p-k)(-4(t(3,1) s(1,5)+t(3,2) s(2,5)) t(6,2) s(1,4) \\
& +2(t(6,1) s(1,5)+t(6,2) s(2,5)) t(1,4) s(3,2)) \\
& +2(t(3,1) s(1,4)+t(3,2) s(2,4)) s(1,5) t(6,2))) \\
& +2(t(6,3) s(3,5)+t(6,4) s(4,5))(t(3,1)(s(1,3) t(3,2)+s(1,4) t(4,2)) s(1,4) \\
& +t(3,2)(s(2,3) t(3,2)+s(2,4) t(4,2)) s(1,4)) \\
& -2 p \cdot k(t(6,3) s(3,5)+t(6,4) s(4,5)) s(1,4) t(3,2) \\
& -(t(3,1) s(1,4)+t(3,2) s(2,4))(t(6,3) s(3,5)+t(6,4) s(4,5)) \\
& (s(1,3) t(3,2)+s(3,4) t(4,2))) \\
& M_{\mu+2}=\frac{-i g^{4} T_{+-+-}^{\mu \gamma^{2}}}{\left(p^{2}-M_{w}^{2}-i \Gamma w M w\right)\left(q^{2}-M_{w}^{2}-i \Gamma w\right.} \overline{\left.M u^{\prime}\right)\left(k_{2}-p\right)^{2}} \\
& T_{+-+-}^{\mu \tau 2}-t(1,6)(s(5,2) t(2.3)-s(5,4) t(4,3), s(4,2)
\end{aligned}
$$




$$
\begin{aligned}
& M_{\mu r 3 \gamma}=\frac{-2 i g^{4} \sin \theta_{W}\left[T_{+-+-}^{\mu+3}+T_{-++-1}^{\mu r 3}\right]}{s\left(q^{2}-M_{W}^{2}-i \Gamma_{W} M_{W}\right)\left(\left(q+p_{2}\right)^{2}-m_{e}^{2}\right)} \\
& M_{\mu \mathrm{r} 3 Z}=\frac{-i g^{4}\left[A_{Z}^{2} T_{+-+-}^{\mu \tau 3}+A_{Z} B_{Z} T_{-++-}^{\mu+3}\right]}{\left.8\left(s-M_{Z}^{2}\right)\left(q^{2}-M_{W}^{2}-i \Gamma_{W} M_{W}\right)\left(q+p_{2}\right)^{2}-m_{e}^{2}\right)} \\
& T_{+-+-}^{\mu r 3}=t(3,1)(s(2,5) t(5,6)+s(2,4) t(4,6)) s(5,4) \\
& T_{-++-}^{\mu+3}=t(3,2)(s(1,5) t(5,6)+s(1,4) t(4,6)) s(5,4) \\
& M_{\mu+4 \gamma}=\frac{-2 i g^{4} \sin \theta_{W}\left[T_{+-t-+}^{\mu \tau 4}+T_{-+t-}^{\mu \tau 4}\right]}{s\left(p^{2}-M_{W}^{2}-i \Gamma W M W\right)\left(\left(p+q_{2}\right)^{2}-m_{e}^{2}\right)} \\
& M_{\mu r 4 Z}=\frac{-i g^{4} \sin \theta_{W}\left[A_{Z}^{2} T_{+-+-}^{\mu r 4}+A_{Z} B_{Z} T_{-++}^{\mu T 4}\right]}{8\left(s-M_{Z}^{2}\right)\left(p^{2}-M_{W}^{2}-i \Gamma_{W} M_{W}\right)\left(\left(p+q_{2}\right)^{2}-m_{e}^{2}\right)} \\
& T_{+-+-}^{\mu r 4}=t(6,3)(s(4,3) t(3,1)+s(4,6) t(6,1)) s(2,5) \\
& T_{-++-}^{\mu+4}=t(6,3)(t(6,2) s(4,6)+t(3,2) s(4,3)) s(1,5) \\
& M_{\mu \tau 5}=\frac{-i g^{4}\left[A_{Z} T_{+-+-}^{\mu \tau 5}+B_{Z} T_{-+++-}^{\mu \tau 5}\right]}{4 \cos ^{2} \theta_{W}\left(s-M_{Z}^{2}\right)\left(q^{2}-1 i_{W}^{2}-i \Gamma_{W} M_{W}\right)\left(p_{1}+q\right)^{2}} \\
& T_{+-+-}^{\mu+5}=t(3,6)(s(5,6) t(6,1)+s(5,3) t(3,1)) s(2,4) \\
& T_{-++-}^{\mu+s}=t(3,6)(s(5,6) t(6,2)+s(5,3) t(3,2)) s(1,4)
\end{aligned}
$$




$$
\begin{gathered}
M_{\mu \tau 6}=\frac{-i g^{4}\left[A_{Z} T_{+-+-}^{\mu r 6}+B_{Z} T_{-++-}^{\mu r 6}\right]}{4 \cos ^{2} \theta_{W}\left(s-M T_{Z}^{2}\right)\left(p^{2}-M_{W}^{2}-i \Gamma_{W} M{ }_{W}\right)\left(q_{1}+p\right)^{2}} \\
T_{+-+-}^{\mu \tau 6}=t(6,1)(s(2,1) t(1,3)-s(2,6) t(6,3)) s(4,5) \\
T_{-++-}^{\mu \tau 6}=t(6,2)(s(1,2) t(2,3)-s(1,6) t(6,3)) s(4,5)
\end{gathered}
$$

$$
\begin{aligned}
M_{\mu e 1 \gamma} & =\frac{i g^{4} \sin ^{2} \theta_{W}\left[T_{+-++}^{\mu e 1}+T_{++++}^{\mu e 1}\right]}{\left(p_{1}-k_{2}\right)^{2}\left(\left(k_{1}-p_{2}\right)^{2}-M_{W}^{2}\right)\left(\left(q^{2}-M_{W}^{2}-i \Gamma_{W} M_{W}\right)\right.} \\
M_{\mu e 1 Z} & =\frac{-i g^{4}\left[A_{Z} T_{+-+-}^{\mu e 1}+B_{Z} T_{++++}^{\mu e 1}\right]}{4\left(\left(p_{1}-k_{2}\right)^{2}-M_{Z}^{2}\right)\left(\left(k_{1}-p_{2}\right)^{2}-M_{W}^{2}\right)\left(\left(q^{2}-M_{W}^{2}-i \Gamma_{W} M_{W}\right)\right.}
\end{aligned}
$$

$$
\begin{aligned}
T_{+-+-}^{\mu e 1}= & 2(t(3,5) s(5,2)+t(3,6) s(6,2)) t(1,6) s(5,4) \\
& -\left(\kappa_{V}+1\right) t(3,1) s(4,2)(t(6,1) s(1,5)-t(6,4) s(4,5)) \\
& -\left(\kappa_{V}+1\right) t(3,6) s(5,2)(t(1,5) s(5,4)+t(1,6) s(6,4)) \\
& -\tilde{\kappa}_{V}(t(6,3) s(2,5)(t(1,2) s(2,4)-t(1,3) s(3,4)) \\
& +t(3,1) s(4,2)(t(6,2) s(2,5)-t(6,3) s(3,5)) \\
& -2 t(6,3) s(2,4)(t(1,2) s(2,5)-t(1,3) s(3,5))) \\
& -\tilde{\lambda}_{V} / 2 M_{W}^{2}(2(t(1,5) s(5,4)+t(1,6) s(6,4)) \\
& (t(6,2)(s(2,4) t(4,3)-s(2,1) t(1,3)) s(2,5) \\
& +t(6,3)(s(3,1) t(1,3)-s(3,4) t(4,3)) s(2,5)) \\
& -2\left(p_{2}-k_{1}\right) \cdot\left(k_{2}-p_{1}\right)(t(1,5) s(5,4)+t(1,6) s(6,4)) t(3,6) s(5,2) \\
& -(t(1,5) s(5,4)+t(1,6) s(6,4))(t(6,2) s(2,5)-t(6,3) s(3,5)) \\
& (t(3,4) s(4,2)-t(3,1) s(1,2)) \\
& \left(p_{2}-k_{1}\right) \cdot q(4(t(1,2) s(2,5)-t(1,3) s(3,5)) t(6,3) s(2,4) \\
& -2(t(6,2) s(2,5)-t(6,3) s(3,5)) t(3,1) s(4,2) \\
& -2(t(1,2) s(2,4)-t(1,3) s(3,4)) t(3,6) s(5,2)
\end{aligned}
$$


$+2(t(6,4) s(4,5)-t(6,1) s(1,5))(t(1,2) s(2,4)-t(1,3) s(3,4)) t(4,3) s(2,4)$ $+2(t(6,4) s(4,5)-t(6,1) s(1,5))(t(1,3) s(3,1)-t(1,2) s(2,1)) t(1,3) s(2,4)$

$-2\left(p_{2}-k_{1}\right) \cdot\left(k_{2}-p_{1}\right)(t(6,4) s(4,5)-t(6,1) s(1,5)) t(3,1) s(4,2)$

$-(t(1,2) s(2,4)-t(1,3) s(4,3))(t(6,4) s(4,5)-t(6,1) s(1,5))$

$(t(3,4) s(4,2)-t(3,1)(s(1,2)))$

$$
\begin{aligned}
& T_{++++}^{\mu e t}=2(s(3,5) t(5,2)+s(3,6) t(6,2)) t(1,6) s(5,4) \\
& -\left(\kappa_{V}+1\right) s(3,4) t(1,2)(t(6,1) s(1,5)-t(6,4) s(4,5)) \\
& -\left(\kappa_{V}+1\right) s(3,5) t(6,2)(t(1,5) s(5,4)+t(1,6) s(6,4)) \\
& -\tilde{\kappa}_{V}(t(6,2) s(3,5)(t(1,2) s(2,4)-t(1,3) s(3,4)) \\
& +t(1,2) s(3,4)(t(6,2) s(2,5)-t(6,3) s(3,5)) \\
& -2 t(6,2) s(3,4)(t(1,2) s(2,5)-t(1,3) s(3,5))) \\
& -\tilde{\lambda}_{v} / 2 M_{W}^{2}(2(t(1,5) s(5,4)+t(1,6) s(6,4)) \\
& (t(6,2)(s(2,4) t(4,2)-s(2,1) t(1,2)) s(3,5) \\
& +t(6,3)(s(3,1) t(1,2) \cdots s(3,4) t(4,2)) s(3,5)) \\
& -2\left(p_{2}-k_{1}\right) \cdot\left(k_{2}-p_{1}\right)(t(1,5) s(5,4)+t(1,6) s(6,4)) t(3,5) s(6,2) \\
& -(t(1,5) s(5,4)+t(1,6) s(6,4))(t(6,2) s(2,5)-t(6,3) s(3,5)) \\
& (s(3,4) t(4,2)-s(3,1) t(1, z)) \\
& \left(p_{2}-k_{1}\right) \cdot q(4(t(1,2) s(2,5)-t(1,3) s(3,5)) t(6,2) s(3,4) \\
& -2(t(6,2) s(2,5)-t(6,3) s(3,5)) t(3,4) s(1,2) \\
& -2(t(1,2) s(2,4)-t(1,3) s(3,4)) t(3,5) s(6,2) \\
& +2(t(6,4) s(4,5)-t(6,1) s(1,5))(t(1,2) s(2,4)-t(1,3) s(3,4)) t(4,2) s(3,4) \\
& +2(t(6,4) s(4,5)-t(6,1) s(1,5))(t(1,3) s(3,1)-t(1,2) s(2,1)) t(1,2) s(3,4) \\
& -2\left(p_{2}-k_{1}\right) \cdot\left(k_{2}-p_{1}\right)(t(6,4) s(4,5)-t(6,1) s(1,5)) t(3,4) s(1,2) \\
& -(t(1,2) s(2,4)-t(1,3) s(3,4))(t(6,4) s(4,5)-t(6,1) s(1,5)) \\
& (s(3,4) t(4,2)-s(3,1) t(1,2)))
\end{aligned}
$$




$$
\begin{aligned}
& M_{\mu e 2 \gamma}=\frac{2 i g^{4} \sin ^{2} \theta_{W}\left[T_{+-+-}^{\mu e 2}+T_{++++}^{\mu e 2}\right]}{\left(p_{1}-k_{2}\right)^{2}\left(\left(q+p_{2}\right)^{2}-M_{W W}^{2}\right)\left(q^{2}-M_{W}^{2}-i\left[w \cdot I_{U} \cdot\right)\right.} \\
& M_{\mu e 2 Z}=\frac{i g^{4}\left[A_{Z}^{2} T_{+-+-}^{\mu e 2}+A^{z} B^{Z} T_{++4}^{\mu e 2}\right]}{8 \cos ^{2} \theta_{W}\left(\left(p_{1}-k_{2}\right)^{2}-M_{Z}^{2}\right)\left(\left(q+p_{2}\right)^{2}-M_{W}^{2}\right)\left(q^{2}-M_{W}^{2}-i \Gamma_{W} \cdot M_{W}\right)} \\
& T_{+-+-}^{\mu e 2}=t(1,3)(s(2,5) t(5,6)+s(2,4) t(4,6)) s(5,4) \\
& T_{++++}^{\mu \mathrm{e} 2}=t(1,2)(s(3,5) t(5,6)+s(3,4) t(4,6)) s(5,4) \\
& M_{\mu e 3}=\frac{-i g^{4}\left[A_{Z} T_{+-++-}^{\mu e 3}+B_{Z} T_{++++}^{\mu e 3}\right]}{4 \cos ^{2} \theta_{W}\left(\left(p_{1}-k_{2}\right)^{2}-M_{Z}^{2}\right)\left(q^{2}-M_{W}^{2}-i \Gamma W M_{W}\right)\left(k_{1}-q\right)^{2}} \\
& T_{+-+-}^{\mu e 3}=t(1,6) s(2,4)(s(5,6) t(6,3)-s(5,1) t(1,3)) \\
& T_{++++}^{\mu e 3}=t(1,6) s(3,4)(s(5,6) t(6,2)-s(5,1) t(1,2)) \\
& M_{\mu e 4 \gamma}=\frac{-2 i g^{4} \sin ^{2} \theta_{W}\left[T_{+-+-}^{\mu e 4}+T_{++++}^{\mu e 4}\right]}{\left(p_{1}-k_{2}\right)^{2}\left(\left(p_{2}-k_{1}+q_{2}\right)^{2}-m_{\mu}^{2}\right)\left(\left(k_{1}-p_{2}\right)^{2}-M_{W}^{2}\right)} \\
& M_{\mu \mathrm{e} Z}=\frac{-i g^{4}\left[A_{Z}^{2} T_{+-4}^{\mu \mathrm{e}}+A_{Z} B_{Z} T_{++++}^{\mu e 4}\right]}{8 \cos ^{2} \theta_{W}\left(\left(p_{1}-k_{2}\right)^{2}-M_{Z}^{2}\right)\left(\left(p_{2}-k_{1}+q_{2}\right)^{2}-m_{\mu}^{2}\right)\left(\left(k_{1}-p_{2}\right)^{2}-M_{W}^{2}\right)} \\
& T_{+-+-}^{\mu e 4}=t(6,1)(s(4,6) t(6,3)-s(4,1) t(1,3)) s(2,5) \\
& T_{++++}^{\mu e 4}=t(6,1)(s(4,6) t(6,2)-s(4,1) t(1,2)) s(3,5)
\end{aligned}
$$




$$
\begin{aligned}
& M_{\mu \mathrm{eS}}=\frac{-i g^{4}\left[A_{Z} T_{+-+-}^{\mu e 5}+B_{Z} T_{++++}^{\mu e 5}\right]}{4 \cos ^{2} \theta_{W}\left(\left(p_{1}-k_{2}\right)^{2}-M_{Z}^{2}\right)\left(\left(k_{1}-p_{2}\right)^{2}-M_{W}^{2}\right)\left(p_{1}-k_{2}+q_{2}\right)^{2}} \\
& T_{+-+-}^{\mu \mathrm{es}}=t(6,3)(s(2,3) t(3,1)+s(2,6) t(6,1)) s(4,5) \\
& T_{++++}^{\mu e 5}=t(6,2)(s(3,2) t(2,1)-s(3,6) t(6,1)) s(4,5) \\
& \begin{aligned}
M_{\mu e 6} & =\frac{-i g^{4} T_{+-+-}^{\mu e 6}}{\left(p_{1}+q\right)^{2}\left(q^{2}-M_{W}^{2}-i \Gamma_{W} M_{W}\right)\left(\left(k_{1}-p_{2}\right)^{2}-M_{W}^{2}\right)} \\
T_{+-+-}^{\mu e 6} & =t(3,6)(s(5,3) t(3,1)+s(5,6) t(6,1)) s(4,2)
\end{aligned} \\
& M_{\mu \mu 1 \gamma}=\frac{i g^{4} \sin ^{2} \theta_{W}\left[T_{+-+}^{\mu \mu 1}+T_{+--+}^{\mu \mu 1}\right]}{\left(q_{1}+p_{1}\right)^{2}\left(\left(k_{1}-p_{2}\right)^{2}-m_{W}^{2}\right)\left(\left(k_{2}-q_{2}\right)^{2}-M_{W}^{2}\right)} \\
& M_{\mu \mu 1 Z}=\frac{-i g^{4}\left[A^{z} T_{+-+-}^{3 \mu \mu 1}+B^{z} T_{+--+}^{\mu \mu 1}\right]}{\left(\left(q_{1}+p_{1}\right)^{2}-M_{Z}^{2}\right)\left(\left(k_{1}-p_{2}\right)^{2}-M_{W}^{2}\right)\left(\left(k_{2}-q_{2}\right)^{2}-M_{W}^{2}\right)}
\end{aligned}
$$

$$
\begin{aligned}
T_{+-+-}^{\mu \mu 1}= & 2 t(6,1) s(4,2)(t(3,1) s(1,5)-t(3,4) s(4,5)) \\
& -\left(\kappa_{V}+1\right)(t(6,3) s(3,2)+t(6,5) s(5,2)) t(3,1) s(4,5) \\
& +\left(\kappa_{V}+1\right) t(6,3) s(5,2)(t(1,3) s(3,4)+t(1,5) s(5,4)) \\
& -\tilde{\kappa}_{V}(t(3,6) s(2,5)(t(1,5) s(5,4)+t(1,3) s(3,4)) \\
& +t(3,1) s(4,5)(t(6,5) s(5,2)+t(6,3) s(3,2)) \\
& -2 t(6,3) s(5,4)(t(1,5) s(5,2)+t(1,3) s(3,2))) \\
& -\bar{\lambda}_{V} / 2 M_{W}^{2}(2(t(1,2) s(2,4)-t(1,6) s(6,4)) \\
& (t(6,5)(s(\dot{3}, 4) t(4,3)-s(5,1) t(1,3)) s(5,2)+t(6,3)(s(3,4) t(4,3)-s(3,1) t(1,3) s(5,2)) \\
& -2\left(p_{2}-k_{1}\right) \cdot\left(q_{1}+p_{1}\right)(t(1,2) s(2,4)-t(1,6) s(6,4)) t(3,6) s(2,5) \\
& -(t(1,2) s(2,4)-t(1,6) s(6,4))(t(6,5) s(5,2)-t(6,3) s(3,2))(t(3,4) s(4,5)-t(3,1) s(1,5)) \\
& +4\left(p_{2}-k_{1}\right) \cdot\left(k_{2}-q_{2}\right)(t(1,5) s(5,2)-t(1,3) s(3,2)) t(6,3) s(5,4)
\end{aligned}
$$


$-2\left(p_{2}-k_{1} j \cdot\left(k_{2}-q_{2}\right)(t(6,5) s(5,2)-t(6,3) s(3,2)) \dot{c}(3,1) s(4,5)\right.$

$-2\left(p_{2}-k_{1}\right) \cdot\left(k_{2}-q_{2}\right)(t(1,5) s(5,4)-t(1,3) s(3,4)) t(3,(i) s(2,5)$

$-2(t(6,4) s(4,2)-t(6,1) s(1,2))(t(1,5)(s(5,4) t(4,3)-s(5,1)(1,3)) s(5,4)$

$+t(1,3)(s(3,4) t(4,3)-s(3,1) t(1,3)) s(5,4))$

$+2\left(q_{2}-k_{1}\right) \cdot\left(q_{1}+p_{1}\right)(t(6,4) s(4,2)-t(6,1) s(1,2)) t(3,1) s(4,5)$

$+(t(1,5) s(5,4)+t(1,3) s(3,4))(t(6,4) s(4,2)-t(6,1) s(1,2))(t(3,4) s(4,5)-t(3,1) s(1,5)))$

$$
\begin{aligned}
& T_{+--+}^{\mu \mu 1}=2 t(6,1) s(4,2)(s(3,1) t(1,5)-s(3.4) t(4.5)) \\
& -(\kappa v+1)(t(6,3) s(3,2)+t(6,5) s(5,2)) s(3,4) t(1,5)) \\
& +(\kappa v+1) t(6,5) s(3,2)(t(1,3) s(3,4)+t(1,5) s(5,4)) \\
& -\tilde{\kappa}_{V}(s(3,4) t(6,5))(t(1,5) s(5,4)+t(1,3) s(3,4)) \\
& +s(3,4) t(1,5))(t(6,5) s(5,2)+t(6,3) s(3,2)) \\
& -2 t(6,5) s(3,4)(t(1,5) s(5,2)+t(1,3) s(3,2))) \\
& -\tilde{\lambda}_{V} / 2 M_{W}^{2}(2(t(1,2) s(2,4)-t(1,6) s(6,4)) \\
& (t(6,5)(s(5,4) t(4,5)-s(5,1) t(1,5)) s(3,2) \\
& +t(6,3)(s(3,4) t(4,5)-s(3,1) t(1,5)) s(3,2)) \\
& -2\left(p_{2}-k_{1}\right) \cdot\left(q_{1}+p_{1}\right)(t(1,2) s(2,4)-t(1,6) s(6,4)) s(3,2) t(6,5) \\
& -(t(1,2) s(2,4)-t(1,6) s(6,4))(t(6,5) s(5,2)-t(6,3) s(3,2)) \\
& (s(3,4) t(4,5)-s(3,1) t(1,5)) \\
& +4\left(p_{2}-k_{1}\right) \cdot\left(k_{2}-q_{2}\right)(t(1,5) s(5,2)-t(1,3) s(3,2)) t(6,5) s(3,4) \\
& -2\left(p_{2}-k_{1}\right) \cdot\left(k_{2}-q_{2}\right)(t(6,5) s(5,2)-t(6,3) s(3,2)) s(3,4) t(5,1) \\
& -2\left(p_{2}-k_{1}\right) \cdot\left(k_{2}-q_{2}\right)(t(1,5) s(5,4)-t(1,3) s(3,4)) t(3,6) s(2,5) \\
& -2(t(6,4) s(4,2)-t(6,1) s(1,2))(t(1,5)(s(5,4) t(4,5)-s(5,1) t(1,5)) s(3,4) \\
& +t(1,3)(s(3,4) t(4,5)-s(3,1) t(1,5)) s(3,4)) \\
& +2\left(q_{2}-k_{1}\right) \cdot\left(q_{1}+p_{1}\right)(t(6,4) s(4,2)-t(6,1) s(1,2)) s(3,4) t(1,5) \\
& +(t(1,5) s(5,4)+t(1,3) s(3,4))(t(6,4) s(4,2)-t(6,1) s(1,2))
\end{aligned}
$$




$$
(s(3,4) t(4,5)-s(3,1) t(1,5)))
$$

$$
\begin{aligned}
& M_{\mu \mu 2 \gamma}= \frac{-2 i g^{4} \sin ^{2} \theta_{W}\left[T_{+-+-}^{2}+T_{+--+}^{\mu \mu 2}\right]}{\left(\left(p_{1}-k_{1}+q_{1}\right)^{2}-m_{e}^{2}\right)\left(\left(k_{2}-q_{2}\right)^{2}-M_{w}^{2}\right)\left(q_{1}+p_{1}\right)^{2}} \\
& M_{\mu \mu 2 Z}= \frac{-i g^{4}\left[A_{Z}^{2} T_{+-+-}^{\mu \mu 2}+A_{Z} B_{Z} T_{+--+}^{\mu \mu 2}\right]}{\left.8 \cos ^{2} \theta_{W}\left(p_{1}-k_{1}+q_{1}\right)^{2}-m_{e}^{2}\right)\left(\left(k_{2}-q_{2}\right)^{2}-M_{w}^{2}\right)\left(\left(q_{1}+p_{1}\right)^{2}-M_{Z}^{2}-i \Gamma_{Z} M_{Z}\right)} \\
& T_{+-+-}^{\mu \mu 2}=t(1,3)(s(5,3) t(3,6)-s(5,1) t(1,6)) s(2,4) \\
& T_{+--+}^{\mu \mu 2}=t(1,5)(s(3,5) t(5,6)-s(3,1) t(1,6)) s(2,4)
\end{aligned}
$$

$$
\begin{aligned}
& M_{\mu \mu 3 \gamma}= \frac{-2 i g^{4} \sin ^{2} \theta_{W}\left[T_{+-+-}^{\mu \mu 3}+T_{+--+}^{\mu \mu 3}\right]}{\left(\left(p_{1}-k_{2}+q_{1}\right)^{2}-m_{e}^{2}\right)\left(\left(k_{1}-p_{2}\right)^{2}-M_{w}^{2}\right)\left(q_{1}+p_{1}\right)^{2}} \\
& M_{i \mu 32}= \frac{-i g^{4}\left[A_{Z}^{2} T_{+-+-}^{\mu \mu 3}+A_{Z} B_{Z} T_{+--+}^{\mu \mu 3}\right]}{8 \cos ^{2} \theta_{W}\left(\left(p_{1}-k_{2}+q_{1}\right)^{2}-m_{e}^{2}\right)\left(\left(k_{1}-p_{2}\right)^{2}-M_{w}^{2}\right)\left(\left(q_{1}+p_{1}\right)^{2}-M_{Z}^{2}-i \Gamma_{Z} M_{Z}\right)} \\
& T_{+-+-}^{\mu \mu 3}=t(6,1)(s(4,2) t(2,3)-s(4,5) t(5,3)) s(5,2) \\
& T_{+--+}^{\mu \mu 3}=t(6,1)(s(4,2) t(2,5)-s(4,3) t(3,5)) s(3,2)
\end{aligned}
$$

$$
M_{\mu \mu 4}=\frac{i g^{4}\left[A_{Z} T_{+-++}^{\mu \mu 4}+B_{Z} T_{+--+}^{\mu \mu 4}\right]}{4 \cos ^{2} \theta_{W}\left(k-q_{2}\right)^{2}\left(\left(k_{2}-q_{2}\right)^{2}-M_{w}^{2}\right)\left(\left(q_{1}+p_{1}\right)^{2}-M_{Z}^{2}-i \Gamma_{Z} M_{Z}\right)}
$$

$$
\begin{aligned}
& T_{+-+-}^{\mu \mu 4}=t(1,6)(s(2,5) t(5,3)+s(2,4) t(4,3)) s(5,4) \\
& T_{+--+}^{\mu \mu 4}=t(1,6)(s(2,3) t(3,5)+s(2,4) t(4,5)) s(3,4)
\end{aligned}
$$




$$
\begin{aligned}
& M_{\mu \mu 5}=\frac{-i g^{4}\left[. A_{Z} T_{+-+}^{\mu \mu 5}+B_{Z} T_{+--+}^{\mu \mu 5}\right]}{4 \cos ^{2} \theta_{W}\left(p_{1}+q\right)^{2}\left(\left(k_{1}-p_{2}\right)^{2}-M_{u 1}^{2}\right)\left(\left(q_{1}+p_{1}\right)^{2}-M_{Z}^{2}-i l_{2} \cdot M_{Z}\right)} \\
& T_{+-+-}^{\mu \mu s}=t(6,3)(s(5,6) t(6,1)+s(5,3) t(3,1)) s(4,2) \\
& T_{+--+}^{\mu \mu 5}=t(6,5)(s(3,5) t(5,1)+s(3,6) t(6,1)) s(4,2) \\
& M_{\mu \mu 6}=\frac{-i g^{4}\left[A_{Z}^{2} T_{+-+-}^{\mu \mu 6}+A_{Z} B_{Z} T_{-++-}^{\mu \mu 6}+A_{Z} B_{Z} T_{+--+}^{\mu \mu 6}+B_{Z}^{2} T_{-+-+}^{\mu \mu \mathrm{i}}+1\right.}{16 \cos ^{4} \theta_{W}\left(p_{1}+q\right)^{2}\left(s-M_{Z}^{2}-i \Gamma_{Z} M_{Z}\right)\left(\left(q_{1}+p_{1}\right)^{2}-M_{Z}^{2}-i \Gamma_{Z} M_{Z}\right)} \\
& T_{+-+-}^{\mu \mu 6}=t(6,3)(s(5,3) t(3,1)+s(5,6) t(6,1)) s(2,4) \\
& T_{-++-}^{\mu \mu 6}=t(6,3)(s(5,3) t(3,2)+s(5,6) t(6,2)) s(1,4) \\
& T_{+-{ }^{\prime}+}^{\mu \mu 6}=t(6,5)(s(3,5) t(5,1)+s(3,6) t(6,1)) s(2,4) \\
& T_{-+-+}^{\mu \mu 6}=t(6,5)(s(3,5) t(5,2)+s(3,6) t(6,2)) s(1,4
\end{aligned}
$$

$$
M_{\mu \mu 7}=\frac{i g^{4}\left[A_{Z}^{2} T_{+-+-}^{\mu \mu 7}+A_{Z} B_{Z} T_{-++-}^{\mu \mu 7}+A_{Z} B_{z} T_{+--+}^{\mu \mu 7}+B_{Z}^{2} T_{-+-+}^{\mu \mu 7}\right]}{16 \cos ^{4} \theta_{W}\left(k-q_{2}\right)^{2}\left(s-M_{Z}^{2}-i \Gamma_{Z} M_{Z}\right)\left(\left(q_{1}+p_{1}\right)^{2}-M_{Z}^{2}-i \Gamma_{Z} M_{Z}\right)}
$$

$$
\begin{aligned}
& T_{+-+-}^{\mu \mu 7}=t(6,1)(s(2,5) t(5,3)+s(2,4) t(4,3)) s(5,4) \\
& T_{-++-}^{\mu \mu 7}=t(6,2)(s(1,5) t(5,3)+s(1,4) t(4,3)) s(5,4) \\
& T_{+--+}^{\mu \mu 7}=t(6,1)(s(2,3) t(3,5)+s(2,4) t(4,5)) s(3,4) \\
& T_{-+-+}^{\mu \mu 17}=t(6,2)(s(1,3) t(3,5)+s(1,4) t(4,5)) s(3,4)
\end{aligned}
$$

$$
\begin{aligned}
M_{\mu \mu 8 \gamma} & =\frac{i g^{4} \sin ^{2} \theta_{W}\left[A_{Z} T_{+-+-}^{\mu \mu 8}+B_{Z} T_{-++-}^{\mu \mu 8}+A_{Z} T_{+--+}^{\mu \mu 8}+B_{Z} T_{-+-+}^{\mu \mu s}\right]}{2 \cos ^{2} \theta_{W}\left(\left(p_{1}+q_{1}-k_{1}\right)^{2}-m_{e}^{2}\right)\left(\left(q_{2}+p_{2}\right)^{2}-M_{Z}^{2}-i \Gamma_{Z} M_{Z}\right)\left(q_{1}+p_{1}\right)^{2}} \\
M_{\mu \mu 8 Z} & =\frac{i g^{4}\left[A_{Z}^{3} T_{+-+-}^{\mu \mu 8}+A_{Z} B_{Z}^{2} T_{-++}^{\mu \mu 8}+A_{Z}^{2} B_{Z} T_{+--+}^{\mu \mu s}+B_{Z}^{3} T_{-+}^{\mu \mu k}\right]}{32 \cos ^{4} \theta_{W}\left(\left(p_{1}+q_{1}-k_{1}\right)^{2}-m_{e}^{2}\right)\left(\left(q_{2}+p_{2}\right)^{2}-M_{Z}^{2}-i \Gamma_{Z} M_{Z}\right)\left(\left(q_{1}+p_{1}\right)^{2}-M_{Z}^{2}-i \Gamma_{Z} M_{Z}\right)}
\end{aligned}
$$




$$
\begin{aligned}
& T_{+-+-}^{\mu \mu ष}=t(1,3)(s(5,1) t(1,6)-s(5,3) t(3,6)) s(4,2) \\
& T_{-++-}^{\mu \mu 8}=s(1,5)(t(3,1) s(1,4)-t(3,5) s(5,4)) t(6,2) \\
& T_{-+-+}^{\mu \mu s}=s(1,3)(t(5,1) s(1,4)-t(5,3) s(3,4)) t(6,2) \\
& T_{+--+}^{\mu \mu s}=t(1,5)(s(3,1) t(1,6)-s(3,5) t(5,6)) s(4,2)
\end{aligned}
$$

$$
\begin{aligned}
& M_{\mu \mu 9 \gamma}= \frac{-i g^{4} \sin ^{2} \theta_{W}\left[A_{Z} T_{+-+-}^{\mu \mu 9}+B_{Z} T_{-++-}^{\mu \mu 9}+A_{Z} T_{+}^{\mu \mu}-++B_{Z} T_{-+-+}^{\mu \mu 9}\right]}{2 \cos ^{2} \theta_{W}\left(\left(p_{1}+q_{1}-k_{2}\right)^{2}-m_{e}^{2}\right)\left(\left(q_{2}+p_{2}\right)^{2}-M_{Z}^{2}-i \Gamma_{Z} M_{Z}\right)\left(q_{1}+p_{1}\right)^{2}} \\
& M_{\mu \mu 9 Z}=\frac{-i g^{4}\left[A_{Z}^{3} T_{+-+-}^{\mu \mu g}+A_{Z} B_{Z}^{2} T_{-++}^{\mu \mu 9}+A_{Z}^{2} B_{Z} T_{+--+}^{\mu \mu 9}+B_{Z}^{3} T_{-+-+}^{\mu \mu 9}\right]}{32 \cos ^{4} \theta_{W}\left(\left(p_{1}+q_{1}-k_{2}\right)^{2}-m_{e}^{2}\right)\left(\left(q_{2}+p_{2}\right)^{2}-M_{Z}^{2}-i \Gamma_{Z} M_{Z}\right)\left(\left(q_{1}+p_{1}\right)^{2}-M_{Z}^{2}-i \Gamma_{Z} M_{Z}\right)} \\
& T_{+-+-}^{\mu \mu 9}=t(1,6)(s(4,2) t(2,3)-s(4,5) t(5,3)) s(5,2) \\
& T_{+++-}^{\mu \mu 9}=s(1,4)(t(6,2) s(2,5)-t(6,3) s(3,5)) t(3,2) \\
& T_{-+-+}^{\mu \mu y}=s(1,4)(t(6,2) s(2,3)-t(6,5) s(5,3)) t(5,2) \\
& T_{+--+}^{\mu \mu 9}=t(1,6)(s(4,2) t(2,5)-s(4,5) t(3,5)) s(3,2)
\end{aligned}
$$

$$
\begin{aligned}
M_{\mu \mu 10 y} & =\frac{-g^{4} \sin ^{2} \theta_{W}\left[A_{Z} T_{+-t-}^{\mu \mu 10}+A_{Z} T_{-++-}^{\mu \mu 10}+B_{Z} T_{-+-t}^{\mu \mu 10}+B_{Z} T_{+--+}^{\mu \mu 10}\right]}{2 \cos ^{2} \theta_{W s} s\left(\left(q+p_{2}\right)^{2}-m_{e}^{2}\right)\left(\left(q_{2}+p_{2}\right)^{2}-M_{Z}^{2}-i \Gamma_{Z} M_{Z}\right)} \\
M_{\mu \mu 10 Z} & =\frac{-g^{4}\left[A_{Z}^{3} T_{+-+-}^{\mu \mu 10}+A_{Z}^{2} B_{Z} T_{-++}^{\mu \mu 10}+B_{Z}^{3} T_{-+-+}^{\mu \mu 10}+A_{Z} B_{Z}^{2} T_{+--+}^{\mu \mu 10}\right]}{32 \cos ^{4} \theta_{W}\left(s-M_{Z}^{2}-i \Gamma_{Z} M_{Z}\right)\left(\left(q+p_{2}\right)^{2}-m_{e}^{2}\right)\left(\left(q_{2}+p_{2}\right)^{2}-M_{Z}^{2}-i \Gamma_{Z} M_{Z}\right)}
\end{aligned}
$$

$$
\begin{aligned}
& T_{+-+-}^{\mu \mu 10}=t(3,1)(s(2,4) t(4,6)+s(2,5) t(5,6)) s(4,5) \\
& T_{-++-}^{\mu \mu 10}=t(3,2)(s(1,4) t(4,6)+s(1,5) t(5,6)) s(4,5) \\
& T_{-+-+}^{\mu \mu 10}=s(3,1)(t(2,5) s(5,4)+t(2,6) s(6,4)) t(6,5) \\
& T_{+--+}^{\mu \mu 10}=s(3,2)(t(1,5) s(5,4)+t(1,6) s(6,4)) t(6,5)
\end{aligned}
$$




$$
\begin{aligned}
& M_{\mu \mu 11 \gamma}=\frac{-i g^{4} \sin ^{2} \theta_{W}\left[A_{Z} T_{+-+-}^{\mu \mu 11}+A_{Z} T_{-++}^{\mu \mu 11}+B_{Z} T_{-+-+}^{\mu \mu 11}+B_{Z} T_{+--+}^{\mu \mu 11}\right]}{2 \cos ^{2} \theta_{W} s\left(\left(p+q_{2}\right)^{2}-m_{E}^{2}\right)\left(\left(q_{z}+p_{2}\right)^{2}-M_{Z}^{2}-i \Gamma_{Z} \cdot I_{Z}\right)} \\
& M_{\mu \mu 11 Z}=\frac{-i g^{4}\left[A_{Z}^{3} T_{+-+-}^{\mu \mu 11}+A_{Z}^{2} B_{Z} T_{-++}^{\mu \mu \mu 1}+B_{Z}^{3} T_{-+-+}^{\mu \mu 11}+A_{Z} B_{Z}^{z} T_{+--+}^{\mu \mu 11}\right]}{32 \cos ^{4} \theta_{W}\left(s-M_{Z}^{2}-i \Gamma_{Z} M_{Z}\right)\left(\left(p+q_{2}\right)^{2}-m_{e}^{2}\right)\left(\left(q_{2}+p_{2}\right)^{2}-M_{Z}^{2}-i \Gamma_{Z} \cdot I_{Z}\right)} \\
& T_{+-+-}^{\mu \mu 11}=t(3,6)(s(4,6) t(6,1)+s(4,3) t(3,1)) s(2,5) \\
& T_{+++-}^{\mu \mu 11}=t(3,6)(s(4,6) t(6,2)+s(4,3) t(3,2)) s(1,5) \\
& T_{-+-+}^{\mu \mu 12}=s(3,4)(t(6,3) s(3,1)+t(6,4) s(4,1)) t(2,5) \\
& T_{+--_{+}}^{\mu \mu 11}=s(3,4)(t(6,3) s(3,2)+t(6,4) s(4,2)) t(1,5)
\end{aligned}
$$

$$
\begin{gathered}
M_{\mu \mu 12}=\frac{-i g^{4} T_{+-+-}^{\mu \mu 12}}{\left(\left(k_{1}-p_{2}\right)^{2}-M_{W}^{2}\right)\left(\left(k_{2}-q_{2}\right)^{2}-M_{W}^{2}\right)\left(p_{1}-k_{2}+q_{2}\right)^{2}} \\
T_{+-+-}^{\mu \mu 12}=t(3,6)(s(2,3) t(3,1)+s(2,6) t(6,1)) s(4,5)
\end{gathered}
$$

$$
\begin{aligned}
M_{e e 1 \gamma} & =\frac{2 i g^{4} \sin ^{2} \theta_{W}\left[T_{+-++}^{e e 1}+T_{-}^{e e 1}--\right]}{\left(\left(p_{2}+p_{1}\right)^{2}-M_{W}^{2}\right)\left(k_{1}-q_{1}\right)^{2}\left(\left(k_{2}-q_{2}\right)^{2}-M_{W}^{2}\right)} \\
M_{e e 1 Z} & =\frac{-i g^{4}\left[A_{Z} T_{+-+}^{e e 1}+B_{Z} T_{-}^{e e 1}--\right]}{\left.2\left(p_{2}+p_{1}\right)^{2}-M_{W}^{2}\right)\left(\left(k_{1}-q_{1}\right)^{2}-M_{Z}^{2}\right)\left(\left(k_{2}-q_{2}\right)^{2}-M_{W}^{2}\right)}
\end{aligned}
$$

$$
\begin{aligned}
T_{+-+}^{e e 1}= & -2 t(3,6) s(2,4)(t(1,3) s(3,5)-t(1,4) s(4,5) \\
& +(\kappa V+1)(t(6,3) s(3,2)+t(6,4) s(4,2)) t(1,3) s(4,5) \\
& -(\kappa V+1)(t(3,1) s(1,4)-t(3,5) s(5,4)) t(1,6) s(2,5) \\
& -\tilde{\kappa} v(t(1,6) s(2,5)(t(3,1) s(1,4(-t(3,5) s(5,4)) \\
& +t(1,3) s(4,5)(t(6,1) s(1,2)-t(6,5) s(5,2)) \\
& -2 t(6,1) s(5,4)(t(3,1) s(1,2)-t(3,5) s(5,2)))
\end{aligned}
$$


$-\tilde{\lambda}_{V} / 2 M_{W}^{2}(2(t(3,2) s(2,4)-t(3,6) s(6.4))$

$(t(6,5)(s(5,3) t(3,1)+s(5,4) t(4,1)) s(5,2)-t(6,1)(s(1,3) t(3,1)-s(1,4) t(4,1)) s(5,2)\}$

$+2\left(k_{1}-q_{1}\right) \cdot p(t(3,2) s(2,4)-t(3,6) s(6,4)) t(1,6) s(2,5)$

$+(t(3,2) s(2,4)-t(3,6) s(6,4))(t(6,1) s(1,2)-t(6,5) s(5,2))(t(1,3) s(3,5)+t(1,4) s(4,5)\}$

$-4\left(k_{2}-q_{2}\right) \cdot p(t(3,1) s(1,2)-t(1,5) s(5,2)) t(6,1) s(5,4)$

$+2\left(k_{2}-q_{2}\right) \cdot p(t(6,1) s(1,2)-t(6,5) s(5,2)) t(1,3) s(4,5)$

$+2\left(k_{2}-q_{2}\right) \cdot p(t(3,1) s(1,4)-t(3,5) s(5,4)) t(1,6) s(2,5)$

$+2(t(6,3) s(3,2)-t(6,4) s(4,2))(t(3,1)(s(1,3) t(3,1)+s(1,4) t(4,1)) s(5,4)$

$-t(3,5)(s(5,3) t(3,1)+s(5,4) t(4,1)) s(5,4))$

$-2\left(k_{1}-q_{1}\right) \cdot p(t(6,3) s(3,2)+t(6,4) s(4,2)) t(1,3) s(4,5)$

$-(t(6,3) s(3,2)+t(6,4) s(4,2))(t(3,1) s(1,4)-t(3,5) s(5,4))$

$(t(1,3) s(3,5)+t(1,4) s(4,5)))$

$$
\begin{aligned}
T_{-e 1}= & -2 t(3,6) s(2,4)(s(1,3) t(3,5)-s(1,4) t(4,5) \\
& +\left(\kappa_{V}+1\right)(t(6,3) s(3,2)+t(6,4) s(4,2)) s(1,4) t(3,5) \\
& -\left(\kappa_{V}+1\right)(t(3,1) s(1,4)-t(3,5) s(5,4)) s(1,2) t(6,5) \\
& -\tilde{\kappa}_{V}(t(1,2) s(6,5)(t(3,1) s(1,4(-t(3,5) s(5,4)) \\
& +s(1,4) t(3,5)(t(6,1) s(1,2)-t(6,5) s(5,2)) \\
& -2 t(6,5) s(1,4)(t(3,1) s(1,2)-t(3,5) s(5,2))) \\
& -\lambda_{V} / 2 M_{W}^{2}(2(t(3,2) s(2,4)-t(3,6) s(6,4)) \\
& (t(6,5)(s(5,3) t(3,5)+s(5,4) t(4,5)) s(1,2)-t(6,1)(s(1,3) t(3,5)+s(1,4) t(4,5)) s(1,2)) \\
& +2\left(k_{1}-q_{1}\right) \cdot p(t(3,2) s(2,4)-t(3,6) s(6,4)) s(1,2) t(6,5) \\
& +(t(3,2) s(2,4)-t(3,6) s(6,4))(t(6,1) s(1,2)-t(6,5) s(5,2))(s(1,3) t(3,5)+s(1,4) t(4,5)) \\
& -4\left(k_{2}-q_{2}\right) \cdot p(t(3,1) s(1,2)-t(1,5) s(5,2)) t(6,5) s(1,4) \\
& +2\left(k_{2}-q_{2}\right) \cdot p(t(6,1) s(1,2)-t(6,5) s(5,2)) s(1,4) t(3,5) \\
& +2\left(k_{2}-q_{2}\right) \cdot p(t(3,1) s(1,4)-t(3,5) s(5,4)) s(1,2) t(6,5) \\
& +2(t(6,3) s(3,2)-t(6,4) s(4,2))(t(3,1)(s(1,3) t(3,5)+s(1,4) t(4,5)) s(1,4)
\end{aligned}
$$




$$
\begin{aligned}
& -t(3,5)(s(5,3) t(3,5)+s(5,4) t(4,5)) s(1,4)) \\
& -2\left(k_{1}-q_{1}\right) \cdot p(t(6,3) s(3,2)+t(6,4) s(4,2)) s(1,4) t(3,5) \\
& -(t(6,3) s(3,2)+t(6,4) s(4,2))(t(3,1) s(1,4)-t(3,5) s(5,4)) \\
& (s(1,3) t(3,5)+s(1,4) t(4,5)))
\end{aligned}
$$

$$
\begin{aligned}
& M_{e e 2 \gamma}=\frac{-2 i g^{4} \sin ^{2} \theta_{W}\left[T_{+-e 2}^{e+-}+T_{---1}^{e 2}\right]}{\left(p^{2}-M_{W}^{2}-i \Gamma_{W} M_{W}\right)\left(\left(p+q_{2}\right)^{2}-m_{e}^{2}\right)\left(k_{1}-q_{1}\right)^{2}} \\
& M_{e e 2 Z}=\frac{-i g^{4}\left[A_{Z}^{2} T_{+-+}^{e e 2}+-A_{Z} B_{Z} T_{--}^{e e}-1\right.}{8 \cos ^{2} \theta_{W}\left(p^{2}-M_{W}^{2}-i \Gamma_{W} M_{W}\right)\left(\left(p+q_{2}\right)^{2}-m_{c}^{2}\right)\left(\left(k_{1}-q_{1}\right)^{2}-M_{Z}^{2}\right)} \\
& T_{+-+-}^{e e 2}=t(6,3)(s(4,3) t(3,1)+s(4,6) t(6,1)) s(5,2) \\
& T_{----}^{e e 2}=t(6,3)(s(4,3) t(3,5)+s(4,6) t(6,5)) s(1,2) \\
& M_{e e 3}=\frac{i g^{4} T_{+-+}^{e e 3}}{\left(p^{2}-M_{W}^{2}\right)\left(k-q_{2}\right)^{2}\left(\left(k_{2}-q_{2}\right)^{2}-M_{W}^{2}\right)} \\
& T_{+-+-}^{\text {ee3 }}=t(1,6)(s(2,4) t(4,3)+s(2,5) t(5,3)) s(4,5) \\
& M_{e e 4 \gamma}=\frac{-2 i g^{4} \sin ^{2} \theta_{W}\left[T_{+-+-}^{e e 4}+T_{--4}^{e e}\right]}{\left(\left(k_{2}-q_{2}\right)^{2}-M_{W}^{2}\right)\left(\left(p_{1}+q_{1}-k_{1}\right)^{2}-m_{e}^{2}\right)\left(k_{1}-q_{1}\right)^{2}} \\
& M_{e e 4 Z}=\frac{-g^{4}\left[A_{Z}^{2} T_{+-++}^{e e 4}+A_{Z} B_{Z} T_{--4}^{e e}\right]}{\left.8 \cos ^{2} \theta_{W}\left(k_{2}-q_{2}\right)^{2}-M_{W}^{2}\right)\left(\left(p_{1}+q_{1}-k_{1}\right)^{2}-m_{e}^{2}\right)\left(\left(k_{1}-q_{1}\right)^{2}-M_{Z}^{2}\right)} \\
& T_{+-+-}^{e t 4}=t(3,1)(s(5,3) t(3,6)-s(5,1) t(1,6)) s(2,4) \\
& T_{---}^{\text {ee4 }}=t(3,5)(s(1,3) t(3,6)+s(1,5) t(5,6)) s(2,4)
\end{aligned}
$$




$$
\begin{aligned}
& M_{e e s}=\frac{-i g^{4}\left[A_{Z} T_{+}^{e e 5}+-+B_{Z} T_{-}^{e e 5}--\right]}{4 \cos ^{2} \theta_{W}\left(\left(k_{2}-q_{2}\right)^{2}-M_{W}^{2}\right)\left(p_{1}+q_{2}-k_{2}\right)^{2}\left(\left(k_{1}-q_{1}\right)^{2}-M_{Z}^{2}\right)} \\
& T_{+-+-}^{\text {ees }}=t(3,6)(s(2,3) t(3,1)+s(2,6) t(6,1)) s(5,4) \\
& T_{---}^{\mathrm{ees}}=\iota(3,6)(s(2,3) t(3,5)+s(2,6) t(6,5)) s(1,4) \\
& M_{e e 6}=\frac{-i g^{4}\left[A_{Z} T_{+-+-}^{e e 6}+B_{Z} T_{---\epsilon}^{e e}-\right]}{4 \cos ^{2} \theta_{W}\left(\left(k_{1}-q_{1}\right)^{2}-M_{Z}^{2}\right)\left(k_{1}-q\right)^{2}\left(p^{2}-M_{W}^{2}-i \Gamma_{W} M_{W}\right)} \\
& T_{+-+-}^{\text {eé }}=t(6,1)(s(5,2) t(2,3)-s(5,4) t(4,3)) s(4,2) \\
& T_{-}^{\text {eeG }}=t(6,5)(s(1,2) t(2,3)+s(1,4) t(4,3)) s(4,2) \\
& M_{e e 7}=\frac{-i g^{4}\left[A_{Z}^{2} T_{+-+}^{e e 7}+A_{Z} B_{Z} T_{++++}^{e e 7}+A_{Z} B_{Z} T_{--Z}^{\text {ee? }}+B_{Z}^{2} T_{-+}^{e e 7}\right]}{16 \cos ^{4} \theta_{W}\left(\left(k_{1}-q_{1}\right)^{2}-M_{Z}^{2}\right)\left(k_{1}-q\right)^{2}\left(\left(p-k_{2}\right)^{2}-M_{Z}^{2}\right)} \\
& T_{+-+-}^{e e 7}=t(6,1)(s(5,1) t(1,3)+s(5,6) t(6,3)) s(2,4) \\
& T_{++++}^{\text {ee7 }}=t(6,1)(-s(5,1) t(1,2)+s(5,6) t(6,2)) s(3,4) \\
& T_{-\mathrm{ee7}}=t(6,5)(s(1,5) t(5,3)+s(1,6) t(6,3)) s(2,4) \\
& \Gamma_{-+-+}^{\text {ee? }}=t(6,5)(s(1,5) t(5,2)+s(1,6) t(6,2)) s(3,4)
\end{aligned}
$$




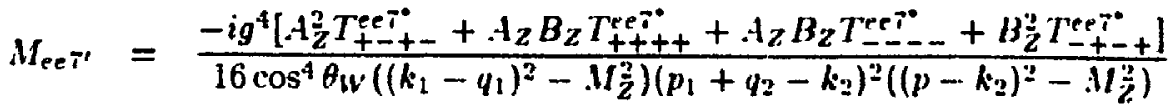

$$
\begin{aligned}
& T_{+-+-}^{\text {ee }}=t(6,3)(s(2,3) t(3,1)+s(2,6) t(6,1)) s(5,4) \\
& T_{++++}^{e e 7^{\circ}}=t(6,2)(s(3,6) t(6,1)+s(3,6) t(6,1)) s(5,4) \\
& T_{-e T^{*}}^{e-}=t(6,3)(s(2,3) t(3,5)+s(2,6) t(6,5)) s(1,4) \\
& T_{-+-+}^{e e \tau^{*}}=t(6,2)(s(3,2) t(2,5)-s(3,6) t(6,5)) s(1,4)
\end{aligned}
$$

$$
\begin{aligned}
M_{e e 8 \gamma} & =\frac{i g^{4} \sin ^{2} \theta_{W}\left[A_{Z} T_{+-+-}^{e+8}+A_{Z} T_{++++}^{e e 8}+B_{Z} T_{-e 8}^{e e-}+B_{Z} T_{-+-+}^{e e 8}\right]}{2 \cos ^{2} \theta_{W}\left(\left(q+p_{2}\right)^{2}-m_{e}^{2}\right)\left(\left(q_{2}+p_{2}\right)^{2}-M_{Z}^{2}-i \Gamma_{Z} M_{Z}\right)\left(p_{1}-k_{2}\right)^{2}} \\
M_{e e 8 Z}= & \frac{i g^{4}\left[A_{Z}^{3} T_{+-++}^{e e 8}+A_{Z}^{2} B_{Z} T_{++++}^{e e 8}+A_{Z} B_{Z}^{2} T_{-}^{e e 8}-+B_{Z}^{3} T_{-+-+}^{e e 8}\right]}{32 \cos ^{4} \theta_{W}\left(\left(q+p_{2}\right)^{2}-m_{e}^{2}\right)\left(\left(q_{2}+p_{2}\right)^{2}-M_{Z}^{2}-i \Gamma_{Z} M_{Z}\right)\left(\left(p_{1}-k_{2}\right)^{2}-M_{Z}^{2}\right)}
\end{aligned}
$$

$$
\begin{aligned}
& T_{+-+-}^{e e 8}=t(1,3)(s(2,5) t(5,6)+s(2,4) t(4,6)) s(4,5) \\
& T_{++++}^{e e 8}=t(1,2)(s(3,5) t(5,6)+s(3,4) t(4,6)) s(4,5) \\
& T_{--e 8}^{\text {ces }}=s(1,2)(t(3,5) s(5,4)+t(3,6) s(6,4)) t(6,5) \\
& T_{-+-+}^{\text {ees }}=s(1,3)(t(2,5) s(5,4)+t(2,6) s(6,4)) t(6,5)
\end{aligned}
$$

$$
\begin{aligned}
M_{e e 9 \gamma} & =\frac{-i g^{4} \sin ^{2} \theta_{W}\left[A_{Z} T_{+-+-}^{e e 9}+B_{Z} T_{++++}^{e e 9}+A_{Z} T_{----}^{e e 9}+B_{Z} T_{-+-+}^{e e g}\right]}{2 \cos ^{2} \theta_{W}\left(\left(p+q_{2}\right)^{2}-m_{e}^{2}\right)\left(\left(q_{2}+p_{2}\right)^{2}-M_{Z}^{2}-i \Gamma_{Z} M_{Z}\right)\left(k_{1}-q_{1}\right)^{2}} \\
M_{e e 9 Z}= & \frac{-i g^{4}\left[A_{Z}^{3} T_{+-+-}^{e e 9}+A_{Z}^{2} B_{Z} T_{+++++}^{e e 9}+A_{Z} B_{Z}^{2} T_{----}^{e e 9}+B_{Z}^{3} T_{-+-+}^{e e g}\right]}{32 \cos ^{4} \theta_{W}\left(\left(p+q_{2}\right)^{2}-m_{e}^{2}\right)\left(\left(q_{2}+p_{2}\right)^{2}-M_{Z}^{2}-i \Gamma_{Z} M_{Z}\right)\left(\left(k_{1}-q_{1}\right)^{2}-M_{Z}^{2}\right)}
\end{aligned}
$$

$$
\begin{aligned}
& T_{+++-}^{e e s}=t(3,6)(s(4,6) t(6,1)+s(4,3) t(3,1)) s(5,2) \\
& T_{++++}^{\text {eeg }}=s(3,4)(t(6,4) s(4,5)+t(6,3) s(3,5)) t(1,2) \\
& T_{-}^{e e 9}=t(3,6)(s(4,6) t(6,5)+s(4,3) t(3,5)) s(1,2) \\
& T_{-+-+}^{e e 9}=s(3,4)(t(6,4) s(4,1)+t(6,3) s(3,1)) t(5,2)
\end{aligned}
$$




$$
\begin{aligned}
& M_{e+10 \gamma}=\frac{-i g^{4} \sin ^{2} \theta_{W}\left[A_{Z} T_{+-+}^{e e 10}+A_{Z} T_{+++++}^{e+10}+B_{Z} T_{-10}^{e e 10}+B_{Z} T_{-+-+}^{e e 10}\right]}{2 \cos ^{2} \theta_{W}\left(\left(p_{1}+q_{1}-k_{2}\right)^{2}-m_{e}^{2}\right)\left(\left(q_{2}+p_{2}\right)^{2}-M_{Z}^{2}-i \Gamma_{Z} M_{Z}\right)\left(p_{1}-k_{2}\right)^{2}}
\end{aligned}
$$

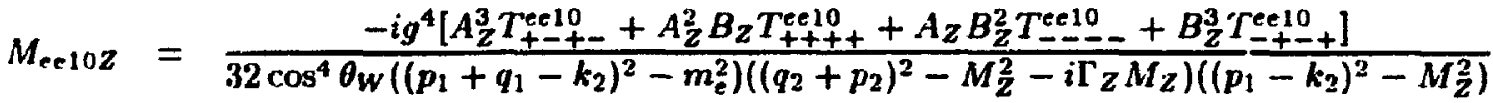

$$
\begin{aligned}
& T_{+-+-}^{\text {ee10 }}=t(1,6)(s(4,1) t(1,3)-s(4,6) t(6,3)) s(2,5) \\
& T_{++++}^{\text {ee10 }}=t(1,6)(s(4,1) t(1,2)-s(4,6) t(6,2)) s(3,5) \\
& T_{--10}^{\text {ee }}=s(1,4)(t(6,1) s(1,2)-t(6,4) s(4,2)) t(3,5) \\
& T_{-+-+}^{\text {ee10 }}=s(1,4)(t(6,1) s(1,3)-t(6,4) s(4,3)) t(2,5)
\end{aligned}
$$

$$
\begin{aligned}
& M_{\text {eel1 }}=\frac{-i g^{4} \sin ^{2} \theta_{W}\left[A_{Z} T_{+-t}^{e e 21}+B_{Z} T_{++t+}^{e e 11}+A_{Z} T_{-}^{e e 11}-+B_{Z} T_{-+++}^{e e 11}\right]}{2 \cos ^{2} \theta_{W}\left(\left(p_{1}+q_{1}-k_{2}\right)^{2}-m_{e}^{2}\right)\left(\left(q_{2}+p_{2}\right)^{2}-M_{Z}^{2}-i \Gamma_{Z} M_{Z}\right)\left(k_{1}-q_{1}\right)^{2}} \\
& M_{e e 11 Z}=\frac{-i g^{4} \sin ^{2} \theta_{W}\left[A_{Z}^{3} T_{+-++}^{e e 11}+A_{Z} B_{Z}^{2} T_{+++++}^{e e 11}+A_{Z}^{2} B_{Z} T_{--11}^{e+1}+B_{Z}^{3} T_{-++}^{e+1}\right]}{32 \cos ^{4} \theta_{W}\left(\left(p_{1}+q_{1}-k_{2}\right)^{2}-m_{e}^{2}\right)\left(\left(q_{2}+p_{2}\right)^{2}-M_{Z}^{2}-i \Gamma_{2} M_{Z}\right)\left(\left(k_{1}-q_{1}\right)^{2}-M_{Z}^{2}\right)}
\end{aligned}
$$

$$
\begin{aligned}
& T_{+-+-}^{e e 11}=t(3,1)(s(5,3) t(3,6)-s(5,1) t(1,6)) s(4,2) \\
& T_{++++}^{\text {eel1 }}=s(3,5)(t(1,3) s(3,4)+t(1,5) s(5,4)) t(6,2) \\
& T_{-}^{\text {ee11 }}=t(3,5)(s(1,3) t(3,6)+s(1,5) t(5,6)) s(4,2) \\
& T_{-+-+}^{\text {eet1 }}=s(3,1)(t(5,3) t(3,4)-s(5,1) t(1,6)) s(6,2)
\end{aligned}
$$




\section{Appendix E}

\section{Monte Carlo Algorithm}

For a process with invariant matrix elen'ent squared $|M|^{2}$, we must evaluate the multi-dimensional phase space integral

$$
\sigma=\int|M|^{2} \prod \frac{d^{3} p_{i}}{(2 \pi)^{3} 2 E_{i}}(2 \pi)^{4} \delta^{4}\left(p_{0}-\sum p_{i}\right)
$$

to determine the cross section. With $(3 \cdot n-4)$ degrees of freedom (where $n$ is the number of final state particles), direct numerical calculation of such an integral is impractical for large $n$. The Monte Carlo approach is to approximate the precise value for the integral by sampling the integrand $\mathbf{N}$ times and then taking the average:

$$
\sigma=\int|M|^{2} d(L I P S) \sim \frac{1}{N} \frac{|M|^{2}\left(x_{1}(i), \ldots, x_{n}(i)\right)}{p\left(x_{1}(i), \ldots, x_{n}(i)\right)}
$$

Where $x_{1}(i)$ is the ith sample of a random variable $x_{1}$ distributed over the range $(0,1)$ with dersity function $p(x)$. The integration limits $(0,1)$ are chosen for convenience; physical variables are rescaled to fit. Each single evaluation of $|M|^{2}\left(x_{1}(i), \ldots, x_{n}(i)\right)$ is a crude estimator of the cross section $\sigma$; the average of $\mathrm{N}$ such evaluations is a much better estimator, with the error in estimating $\sigma$ going down like $1 / \sqrt{N}$. In the simplest form of Monte Carlo integration, the random integration points are uniformly distributed i.e., $p(x)=$ constant. In the VEGAS algorithm [59], the density $p(x)$ is modified between succesive iterations so as to minimize the uncertainty in the integral estimate. VEGAS makes $m$ estimates of the integral, $S_{\alpha \alpha=1}^{m}$, each iteration using $N$ 
evaluations of the integrand. These $m$ estimates are combined to give a cumulative estimate $\bar{S}$ :

$$
\sigma \sim \bar{S}=\bar{\sigma}^{2} \sum \frac{S_{\alpha}}{\sigma_{\alpha}^{2}}
$$

where $\sigma_{\alpha}$ is the approximate uncertainty in $S_{\alpha}$

$$
\sigma_{a}^{2}=\frac{1}{N-1} \frac{1}{N} \frac{|M|^{2}\left(\vec{x}_{i}\right)}{p\left(\bar{x}_{i}\right)}-S_{\alpha}^{2}
$$

and where $\bar{\sigma}$ is the approximate uncertainty in $\bar{S}$ :

$$
\frac{1}{\bar{\sigma}^{2}}=\Sigma \frac{1}{\sigma_{\alpha}^{2}}
$$

Uniformly distributed random points are employed in the first iteration, giving $S_{\alpha}$ and $\sigma_{\alpha}$. In subsequent iterations, the density $p\left(x_{i}\right)$ is modified so as to concentrate sample points in the regions of phase space where $|M|^{2}$ is largest. Thus, even though $N$ is unchanged, $\sigma_{\alpha}^{2}$ is reduced over several iterations, and the estimates of the integral $\bar{S}$ are progressively improved.

For the four lepton production processes $e^{+} e^{-}+l^{+} \nu l^{\prime-} \bar{\nu}$, we have an eight dimensional phase space integral to evaluate.

$$
\sigma=\int|M|^{2} \prod_{i=1}^{4} \frac{d^{3} p_{i}}{(2 \pi)^{3} 2 E_{i}}(2 \pi)^{4} \delta^{4}\left(p_{0}-\sum p_{i}\right)
$$

For the sake of parametrizing the integration variables, we picture the process schematically as shown in Figure (E.1), where the four final state leptons are assumed to be created in pairs.

Depending on the process, a $l^{-} \bar{\nu}\left(l^{+} \nu\right)$ pair or a $l^{-} l^{+}(\bar{\nu} \nu)$ pair is created at the secondary vertices, with invariant masses of $M_{12}$ and $M_{34}$, respectively. The phase space integral can then be expressed as

$$
\sigma=\frac{1}{(2 \pi)^{8}} \int d M_{12}^{2} \int d M_{34}^{2} R_{2}\left(s, M_{12}^{2}, M_{34}^{2}\right) R_{2}\left(M_{12}^{2}, m_{1}^{2}, m_{2}^{2}\right) R_{2}\left(M_{134}^{2}, m_{3}^{2}, m_{4}^{2}\right)|M|^{2}
$$

where the two point function $R_{2}$ is given by

$$
R_{2}(a, b, c)=\frac{\lambda^{1 / 2}(a, b, c)}{8 a} \int d \Omega
$$




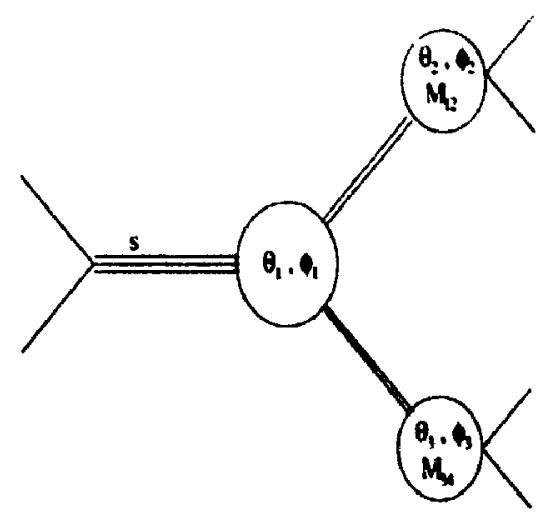

Figure E.1: Schematic Diagram of Four Lepton Production Process

with

$$
\lambda(a, b, c)=a^{2}+b^{2}+c^{2}-2 a b-2 a c-2 b c
$$

In the massless limit $m_{1}=m_{2}=m_{3}=m_{4}=0$, and so

$$
\begin{aligned}
& R_{2}\left(M_{12}^{2}, m_{1}^{2}, m_{2}^{2}\right)=\frac{1}{8} \int d \Omega \\
& R_{2}\left(M_{34}^{2}, m_{3}^{2}, m_{4}^{2}\right)=\frac{1}{8} \int d \Omega
\end{aligned}
$$

Simplifying and rearranging, we find

$$
\sigma=\frac{1}{(2 \pi)^{8}} \int d M_{12} \int d M_{34} \int d \Omega \int d \Omega \int d \Omega|M|^{2} \frac{\lambda^{1 / 2}\left(8, M_{12}^{2}, M_{34}^{2}\right)}{128 s} M_{12} M_{34}
$$

We now rescale the integration variables in terms of the randomly generated $x_{i}(i=1, \ldots, 8)$, where $0<x_{i}<1$. For each angular integration $d \Omega$ we define

$$
\begin{aligned}
\phi_{i} & =2 \pi x_{i} \\
\cos \theta_{i} & =1-2 x_{i+3}
\end{aligned}
$$

and for the invariant mass integrations

$$
M_{12}=s \cdot x_{7}
$$




$$
M_{34}=\left(s-M_{12}\right) \cdot x_{8}
$$

'The phase space integral becomes

$$
\sigma=\frac{1}{s \cdot 2^{9} \pi^{5}} \int \prod_{i=1}^{8}\left(s-M_{12}\right) M_{12} M_{34} \lambda^{1 / 2}\left(s, M_{13}^{2}, M_{34}^{2}\right)|M|^{2}
$$

For each successive "event", the $x_{i}$ are generated from a random number generator. These $x_{i}$ then determine the six angular and two invariant mass variables through the previous expressions. The momenta and energies of the produced leptons are then determined from the $\theta_{i}, \phi_{1}$ and $M_{12}, M_{34}$, after a boost back to the laboratory frame. With the individual momenta $p_{i}$ determined, the matrix element squared $|M|^{2}$ can then be evaluated. Once weighted by the factor of Eq. (E.10), this is the first estimate of the cross section $\sigma$. Each subsequent estimate will be summed, their average is the best approximation of the true integral. After each iteration of $\mathrm{N}$ events, the density $p(\bar{x})$ is adjusted so that the sample points $\left\{x_{i}\right\}$ in the next iteration are concentrated where $|M|^{2}$ was largest. It was generally observed that 10 iterations of $5,000,000$ events was more than sufficient to provide a stable (no large fluctuations between successive iteration estimates) and reliable (reproducible) approximation of the cross section. Since the optimal density grid was usually achieved before 10 iterations, increasing the number of iterations offered no significant improvement in accuracy and 5,000,000 events in each iteration resulted in smooth distributions.

In addition to total cross section, VEGAS can also evaluate distributions such as

$$
\frac{d \sigma}{d y}
$$

where

$$
\sigma=\int d y \frac{d \sigma}{d y}
$$

and $y$ is some observable of the particle momenta and energies. Over successive integration points, the different values for $y$ are weighted by the calculated $|M|^{2}$ appropriate to the individual $y_{i}$, each weighted event is then binned in the appropriate increment $\Delta y_{i}$. The $d \sigma / d y$ at $y=y_{i}$ is then approximated by

$$
\frac{d \sigma\left(y_{i}\right)}{d y} \sim \frac{\Delta \sigma_{i}}{\Delta y_{i}}
$$

where $\Delta \sigma_{i}$ is the contribution to $\sigma$ coming from the increment $\Delta y_{i}$. We generated distributions 
for both those class of variables that could be experimentally measured. ( these include the differential cross section with respect to the opening angle between the charged leptons, $\theta_{+}$. the angle of each charged lepton with the beam. $\theta_{l+}$ and $\theta_{1-}$, the energy, $E_{l}$, and transverse mumentum, $p_{r}^{l}$, of each charged lepton, the total visible energy, $E_{r}$, total transverse momentum, $P_{r}$, and the invariant mass of the charged lepton pair, $\boldsymbol{M}_{l+l-}$ ) as well as those that, although experimentally unaccessible, provided important tests of the calculation. For instance, any distribution involving the momenta or energies of either or both of the two neutrinos would be experimentally unobservable. However, by looking at the invariant mass and angular distributione of the lepton-neutrino pairs, we effectively examine the distributions of the reconstructed $\mathbf{W}$ bowons (with appropriate cuts on $M_{l-\bar{\nu}}$ and $M_{l+\nu}$ ). Our results can therefore be checked against those of $\mathbf{W}$ pair production work.

As an check on the self consistency of the calculation, we generated various redundant distributions to confirm the symmetry under charge conjugation for the $\mu^{+} \tau^{-}, \mu^{+} \mu^{-}$, and $\mu^{+} \mu^{-}$ channels with SM couplings. Thus the charge conjugate angular distributions in $\cos \theta_{t-}$ und $\cos \theta_{l+}$ were compared. The forward-backward asymmetry for both distributions was calculated as

$$
\begin{aligned}
& A_{F B}^{l^{+}}=\frac{N\left(\cos \theta_{l+}\right)-N\left(\cos \theta_{l+}\right)}{N\left(\cos \theta_{l+}\right)+N\left(\cos \theta_{l+}\right)} \\
& A_{F B}^{l^{-}}=\frac{N\left(\cos \theta_{l-}\right)-N\left(\cos \theta_{l-}\right)}{N\left(\cos \theta_{l-}\right)+N\left(\cos \theta_{l-}\right)}
\end{aligned}
$$

and it was verified that $A_{F B}^{++} \sim A_{F B}^{l^{-}}$, as required by charge conjugation invariance.

In addition to the adaptive VEGAS algorithm, we checked our results against a simpler nonadaptive Monte Carlo. The results of the two were found to be in good agreement, the "brutrforce" program was however less efficient in its use of computing time, an important factor given the number of runs necessary. 


\section{Bibliography}

[1] E. Fermi, Z. Phystk 88 (1934) 161.

[2] T.D. Lee and C.N. Yang, Phys. Rev. 104, 254 (1956).

[3] C.S. Wu et al., Phys. Rev. 105, 1413 (1957).

[4] R.P reynman and M. Gell-Man, Phys. Rev. 109, 193 (1958).

[5] E.C.G. Sudarshan and R. Marshak, Phys. Rev. 109, 1860 (1958).

[6] J. Schwinger, Ann. of Physics 2 (1957) 407.

[7] P.W. Anderson, Phys. Rev. 130 (1963) 439.

[8] P.W. Higgs, Phys. Lett. 12 (1964) 132.

[9] F. Englert and R. Brout, Phys. Rev. Lett. 13 (1964) 321.

[10] S.L. Glashow, Nucl. Phys.22 (1961) 579; S. Weinberg, Phys. Rev. Lett.19 (1967) 1264; A. Salam, Proc. 8th Nobel Symposium, Aspenasgarden, 1968, ed.

[11] G. 't Hooft, Nucl. Phys. B35 (1971) 167; G. 't Hooft, Phys. Lett. 37B (1971) 195; G. 't Hooft, Nucl. Phys. B61 (1973) 455.

[12] P.W. Higgs, Phys. Rev. Lett 13 (1964) 508.

[13] Review of Particle Properties, Phys. Rev., D50, 1173 (1994)

[14] W. Hollik, Status of the Electroweak Standand Model, Proceedings of "Lepton and Photon Interactions", Ithaca, 1993 
[15] M. Davier et al.. Procecdings of the "ECFA Workshop on I.EP 200", edls. A.1Buhm an! W.Hoogland (1987), CERN 87-08.

[16] G.L. Kane, J. Vidal, and C.P. Yuan. Phys. Rev. D39 (1989) 2617.

[17] Workshop on "Phystes and Experiments with Lamear pte" Colluders", Waik, Waa. Ilawaii. 1993 World Scientific, eds. F. Harris et al..

[18] Proc. of the workshop on $e^{+} e^{-}$Collisions at 500 GeV: the Physics Potential, DESY 92-12313. 1992, ed. P. Zerwas.

[19] Proc. of the Workshop on Physics and Experiments with Linear Colliders, Saariselka, Hiilland, 1991 (World Scientific 1992), eds. P. Eerola et al.

[20] K. Hagiwara, R.D. Peccei, D. Zeppenfeld, and K. Hikasa Nucl. Phys. B282, 253 (1987).

[21] G. Couture et al., Phys. Rev. D36, 859 (1987).

[22] G. Couture et al., Phys. Rev. D38, 860 (1988).

[23] T.G. Rizzo and M.A. Samuel, Phys. Rev. D35, 403 (1987).

[24] X.G. He, J.P. Ma, and B.H. McKellar, University of Melbourne preprint, UM-P-92/75.

[25] D. Chang, W.Y. Keung, and J. Liu, Nuc!. Phys., 355, 295 (1991)

[26] G. Belanger et al., Phys. Rev. Lett., 65, 2943 (1990)

[27] K.J.F. Gaemers and G.J. Gounaris. Z. Phys. c1 ,259 (1979).

[28] F. Chen and F.M. Renard, Phys. Rev. D28, 1758 (1984).

[29] C. xotinett, Phys. Kev. D28, 1185 (1983); Phys. Rev. D28, 1192 (1193).

[30] C.L. Bilchak and J.D Stroughair, Phys. Rev. D9, 1881 (1984).

[31] M. Lemoine and M. Veltman, Nucl. Phys. B164, 445 (1980).

[32] M. Phillippe, Phys. Rev. D26, 1558 (1982).

[33] G. Couture, S. Godfrey, and R. Lewis, Phys. Rev. D45, 777 (1992).

[34] C. Gross-Knetter and D. Schildknecht, preprint. 
[35] U. Baur and D. Zeppenfeld, Phys. Lett. B201, 383 (1988).

[36)] (:. Counaris, D.Schildknecht, and F.M. Renard Phys. Lett. B263, 291 (1991).

[37] D. Chang et al., Phys. Rev. D48, 4045 (1993).

[38] A.A. Likhoded et al., preprint, IC/93/288.

[39] K.O. Mikaelian, M.A. Samuel, and D. Sahdev, Phys. Lett. 43, 746 (1979).

[40] U. Baur and D. Zeppenfeld, Nucl. Phys. B308, 127 (1988).

[41] U. Baur and E. Berger, Phys. Rev. D41, 1476 (1990).

[42] M. Samuel et al., Phys. Lett. B280, 124 (1992).

[43] F. Cortes et al., Nucl. Phys. B278, 26 (1986).

[44] 'T.R. Grose and K.O. Mikaelian, Phys. Rev. D23 (1981) 123.

[45] I.F. Ginzburg et ai., Nucl. Phys., B228, 285 (1983).

[46] K.O. Mikaelian, Phys. Rev., D30, 1115 (1984).

[47] J.A. Robinson and T.G. Rizzo, Phys. Rev., D33, 2608 (1986).

[48] G. Couture, S. Godfrey, and P. Kalyniak, Phys. Lett. B218, 361 (1989); Phys. Rev. D39, 3239 (1989); D42, 1841 (1990).

[49] E. Yehudai, Phys. Rev., D41, 33 (1990); 44, 3434 (1991).

[50] S.Y. Choi and F. Schrempf, Phys. Lett. B272 (1991) 149.

[51] U. Baur and D. Zeppenfeld, Nucl. Phys. B325, 253 (1989).

[52] C.S. Kim et al., preprint, YUMS 93-14, KEK-TH!-366.

[53] J. Alliti et al., UA2 Collaboration. Phys. Lett. B27T, 194 (1992).

[54] A.L. Spadafora et al., Do Collaboration, Fermilab preprint FERMILAB-CONF-94-016-E,

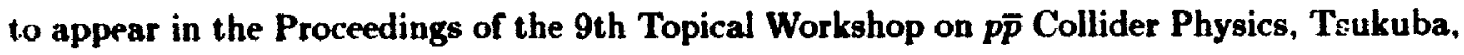
Japan. Ortober 1993. 
[55] F. Abe et al., CDF Collaboration, contributed paper to the $2 i^{\text {th }}$ International ('onference on High Energy Physics. Glasgow, Scotland, 1994: Fermilab preprint, FERMII.AB-( 'ONF: 94/158-E

[56] X-G. He and B. McKellar, Phys. Lett., B320, 165) (1994).

[57] R. Ammar et al., CLEO Collaboration, Phys. Rev. Lrtt., 71, 6ī.4 (1993).

[58] R. Kleiss and W.J. Stirling, Nucl. Phys. B262, 235 (1985).

[59] P. Lepage, Journal of Computational Phystcs 27, 192 (1978).

[60] M. Aguilar-Benitez et al., Phys. Rev., D45, 4365 (1992).

[61] F. Bletzacker and H.T. Nieh, Nucl. Phys. B124, 511 (1977); P. Mery and M. Perotut, Nucl. Phys. B175, 234 (1980).

[62] M. Bilenky et al. Nucl. Phys. B419, 240 (1994).

[G3] G. Couture and S. Godfrey, preprint, OCIP/C, 94-4, UQAM-PHE-94-(19) (1994).

[64] K.J. Abraham, J. Kalinowski, and P. Sciepko, preprint, IFT-94/14.

[65] M. Blondel et al. Study of Reaction $e^{+} e^{-} \rightarrow W^{+} W^{-}$, Aachen ECFA Workshop.

[66] D.H. Perkins, LEP200 Workshop Summary.

[07] O. Philipsen, Z. Phys. C - Particles and Fields 54, 643 (1992).

[68] U. Baur and M.A. Doncheski, Phys. Rev. D46 (1992) 1959.

[69] T.G. Rizzo, Phys. Rev. 32 (1985) 43.

[70] K.R. Barger and M.H. Reno, UIOWA-94-10, IEP-PH-9406331.

[71] J.H. Christenson, J.W. Cronin, V.L. Fitch, R. Turlay, Phys. Rev. Lett. 13 (1964) 138.

[72] F. Boudjema et al., Phys. Rev. D43 (1991) 2223.

[73] M. Kobayashi and M. Maskawa, Proy. Theor. Phys. 49 (1973) 349.

[74] M. Kuroda et al., Phy.. Lett. B190 (1987) 217.

[75] S.D. Rindani, CP Violatzon at Collzders, talk given at WHEPP3, Madras, India, 1993. 
[76] W.Y. Keung, private communication

[77] H... Mani, B. Mukhopadhyaya, and S. Raychaudhari, M.R.I, Allahabad preprint, MRIPHY/9/93(1993).

[78] S. Godfrey and K.A. Peterson, preprint, OCLP/C 92-7

[79] M. Gintner, private communication 

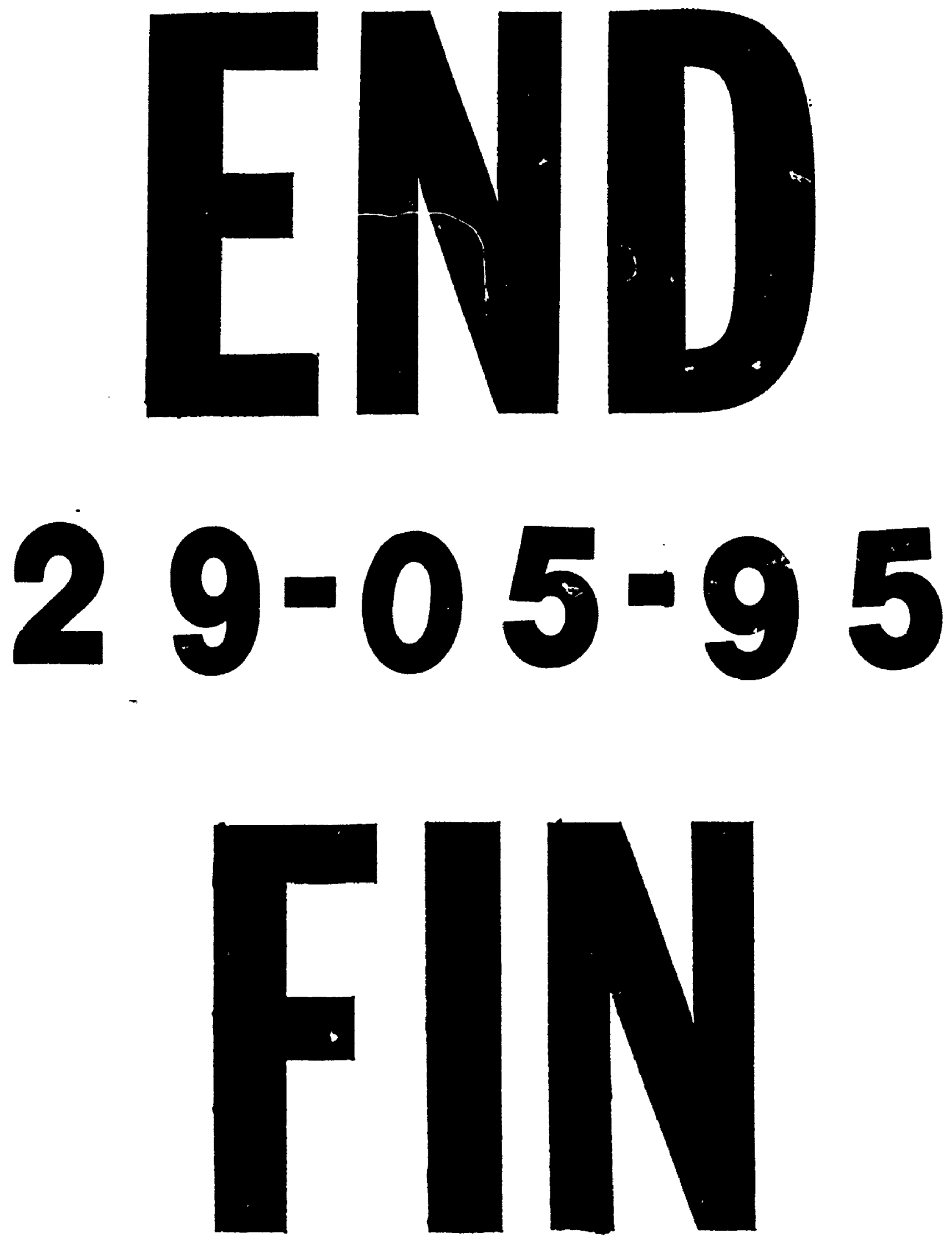